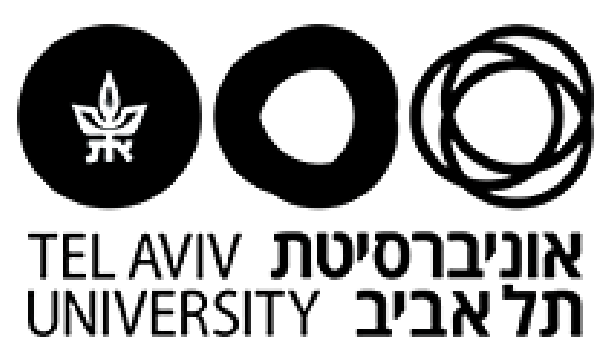

\title{
Short Range Correlated nucleon pairs in Heavy Nuclei
}

Thesis submitted towards the degree of

Doctor of Philosophy

By

Meytal Duer

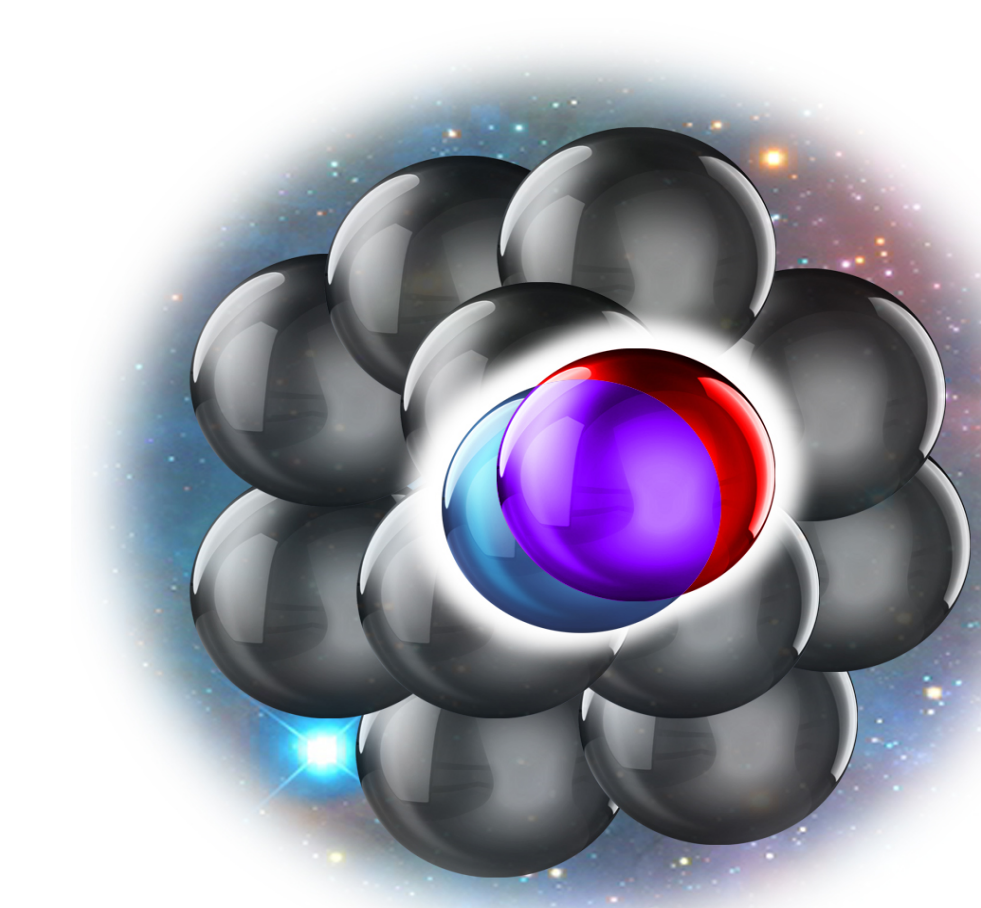

SUBMITTED TO THE SENATE OF TEL-AVIV UNIVERSITY

December 2018

This work was carried under the supervision of

Professor Eli Piasetzky 


\begin{abstract}
The atomic nucleus is one of the densest and most complex quantum-mechanical systems in nature. Nuclei account for nearly all the mass of the visible Universe. The properties of individual nucleons (protons and neutrons) in nuclei can be probed by scattering a high-energy particle from the nucleus and detecting this particle after it scatters, often also detecting an additional knockedout proton. Analysis of electron- and proton-scattering experiments suggests that some nucleons in nuclei form close-proximity neutron-proton pairs with high nucleon momentum, greater than the nuclear Fermi momentum, known as two-nucleon short-range correlated pairs (2N-SRC). However, how excess neutrons in neutron-rich nuclei form such close-proximity pairs remains unclear.

In this thesis we measured protons and, for the first time, neutrons knocked out of medium-to-heavy nuclei by high-energy electrons, using the $A\left(e, e^{\prime} n\right)$ and $A\left(e, e^{\prime} p\right)$ reactions on carbon, aluminum, iron, and lead targets with a $5.01 \mathrm{GeV}$ electron beam and the CEBAF Large Acceptance Spectrometer (CLAS) at the Thomas Jeerson National Accelerator Facility.

We showed that the fraction of high-momentum protons increases markedly with the neutron excess in the nucleus, whereas the fraction of high-momentum neutrons decreases slightly. This effect is surprising because in the classical nuclear shell model, protons and neutrons obey Fermi statistics, having little correlation and mostly filling independent energy shells. These high-momentum nucleons in neutron-rich nuclei are important for understanding nuclear parton-distribution functions (the partial momentum distribution of the constituents of the nucleon) and changes in the quark distributions of nucleons bound in nuclei (the EMC effect). They are also relevant for the interpretation of neutrino-oscillation measurements, and understanding of neutron-rich systems such as neutron stars.
\end{abstract}

We extended the measurement to a triple coincidence $A\left(e, e^{\prime} n p\right)$ and $A\left(e, e^{\prime} p p\right)$ reactions on the nuclei mentioned above. The knocked-out neutrons or protons and scattered electrons were detected in coincidence with a proton recoiling almost back to back to the missing momentum, leaving the residual $A-2$ system at low momentum. Using these data we directly verified, for the first time on neutron-rich nuclei such as lead, that the number of proton-proton SRC pairs is smaller than the number of neutron-proton SRC pairs by about a factor of 20, independent of the neutron excess in the nucleus. 


\section{Contents}

1 Introduction 10

$1.12 \mathrm{~N}-\mathrm{SRC} \ldots \ldots \ldots \ldots \ldots \ldots$

1.1 .1 Quasi-Elastic (QE) Scattering . . . . . . . . . . . . . . . . . . . . . . 12

1.1 .2 Inclusive SRC measurements . . . . . . . . . . . . . . . . . . . . . . . . 13

1.1.3 Double- and Triple-coincidence measurements . . . . . . . . . . . . . . . . . . . . . . . 15

1.2 Energy Sharing in Nuclear Systems . . . . . . . . . . . . . . . . . . . . . . . . . . . 19

2 Experimental Setup 21

2.1 The CEBAF Accelerator . . . . . . . . . . . . . . . . . . . . . . . . . . . . . 21

2.2 The CLAS Detector . . . . . . . . . . . . . . . . . . . . . . . . . . . . . . 21

2.2 .1 Torus Magnet . . . . . . . . . . . . . . . . . . . . . . . . 22

2.2 .2 Drift Chambers . . . . . . . . . . . . . . . . . . . . . . . . . 23

2.2 .3 Cherenkov Counters . . . . . . . . . . . . . . . . . . . . . . 25

2.2 .4 The Time-Of-Flight counters . . . . . . . . . . . . . . . . . . . . . . . 26

2.2 .5 Electromagnetic Calorimeter . . . . . . . . . . . . . . . . . . . . . 27

2.3 The EG2 Experiment . . . . . . . . . . . . . . . . . . . . . . . . . . . 28

3 Data Analysis 30

3.1 Particle Identification . . . . . . . . . . . . . . . . . . . . . . . . . . . 30

$3.1 .1 \quad$ Electron Identification . . . . . . . . . . . . . . . . . . . . . . . . . . . . . 30

3.1 .2 Proton Identification . . . . . . . . . . . . . . . . . . . . . . . . . . . . . . . . 32

3.1 .3 Pions Identification . . . . . . . . . . . . . . . . . . . . . . . . . . . . . . 33

3.1 .4 Vertex Reconstruction . . . . . . . . . . . . . . . . . . . . . . . . . 34

3.1 .5 Neutron Identification, Detection Efficiency, and Momentum Reconstruction Resolution . . . 35

$3.2 \quad$ Identifying $A\left(e, e^{\prime} n\right)$ and $A\left(e, e^{\prime} p\right)$ Quasi-Elastic Events . . . . . . . . . . . . . . . . . . . . 42

$3.2 .1 \quad$ Identifying High-Momentum (SRC) events . . . . . . . . . . . . . . . . . . . . . . . . . . . . 43

3.2 .2 Identifying Low-Momentum (Mean-Field) events . . . . . . . . . . . . . . . . . . . . . . . . . 50

$3.3 \quad$ Identifying the $A\left(e, e^{\prime} n p\right)$ and $A\left(e, e^{\prime} p p\right)$ High-Momentum Events . . . . . . . . . . . . . . . . . . . 57

4 Results

$4.1 \quad$ Extracting $\frac{A\left(e, e^{\prime} N\right)}{C\left(e, e^{\prime} N\right)}$ cross-section ratios . . . . . . . . . . . . . . . . . . . . . . 66

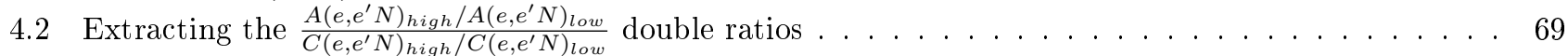

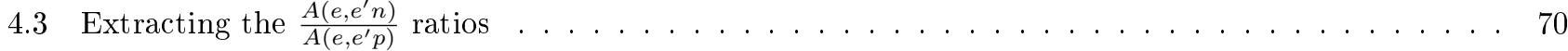

4.4 Triple coincidence ratios . . . . . . . . . . . . . . . . . . . . . . . . . . 75

4.5 Nuclear Transparency ratios . . . . . . . . . . . . . . . . . . . . . . . . . . . 78

5 Summary and Conclusion 8

6 Appendices 90

A ${ }^{27} \mathrm{Al},{ }^{56} \mathrm{Fe}$, and ${ }^{208} \mathrm{~Pb}$ distributions (high-momentum)

B ${ }^{27} \mathrm{Al},{ }^{56} \mathrm{Fe}$, and ${ }^{208} \mathrm{~Pb}$ distributions (low-momentum) 94

$\mathrm{C} \quad{ }^{27} \mathrm{Al},{ }^{56} \mathrm{Fe}$, and ${ }^{208} \mathrm{~Pb}$ distributions (triple coincidence) 
D SCX correction 


\section{List of Figures}

$1 \quad$ The spectroscopic strength for various nuclei, extracted by comparing $A\left(e, e^{\prime} p\right)$ valence knockout

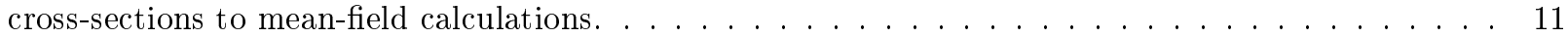

2 Calculation of the expected single-nucleon momentum distributions in ${ }^{12} \mathrm{C}$ based on the independent particle model (dashed red line), and with correlations (solid blue line). . . . . . . . . . . . . 12

3 The electron-nucleon (a) and the electron-nucleus (b) cross-sections represented as a function of the

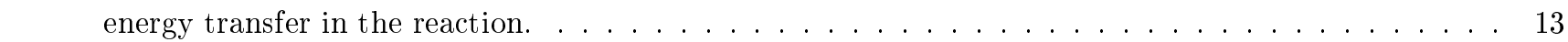

4 The minimum initial momentum of the knocked-out nucleon as a function of $x_{B}$. (a) For deuterium at different $Q^{2}$. (b) For different nuclei at $Q^{2}=2(\mathrm{GeV} / \mathrm{c})^{2}$. . . . . . . . . . . . . . . . 14

5 The per-nucleon $\mathrm{QE}\left(e, e^{\prime}\right)$ cross-section ratios of nucleus $A$ to deuterium $\left(0.8<x_{B}<1.9\right.$, and $\left.Q^{2}>1.5(\mathrm{GeV} / \mathrm{c})^{2}\right) . \ldots \ldots \ldots \ldots \ldots \ldots \ldots \ldots \ldots \ldots$

$6 \quad$ Calculated momentum distributions for different nuclei compared to deuterium [19]. . . . . . . . . 15

$7 \quad$ Distributions of cosine of the relative angle $(\gamma)$ between the reconstructed initial momentum of the

knockout-proton and the recoil nucleon. $\ldots \ldots \ldots \ldots \ldots \ldots \ldots$

$8 \quad$ The ratio of ${ }^{12} \mathrm{C}\left(e, e^{\prime} p N\right)$ double knockout events to ${ }^{12} \mathrm{C}\left(e, e^{\prime} p\right)$ single knockout events as a function

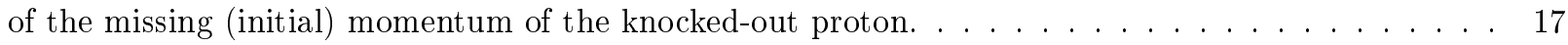

$9 \quad$ Top and middle panels: the ${ }^{4} \mathrm{He}\left(e, e^{\prime} p N\right) /{ }^{4} \mathrm{He}\left(e, e^{\prime} p\right)$ ratios, as a function of the knocked-out proton

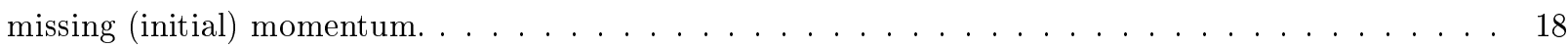

$10 \quad$ The extracted fractions of $n p$ (top) and $p p$ (bottom) SRC pairs from the sum of all SRC pairs in nuclei. 18

11 Schematic layout of JLab accelerator site (before the $12 \mathrm{GeV}$ upgrade). . . . . . . . . . . . . . . 21

12 Center - Two segments of the CLAS spectrometer. Also shown are electrons traveling with energies of up to $6 \mathrm{GeV}$ hitting a nucleus, and knocking out individual protons and neutrons. . . . . . . . . 22

13 Schematic diagram of CLAS six superconducting coils that create the toroidal magnet. . . . . . . . . 23

14 The three regions of the CLAS DC, and the torus magnetic field strength. . . . . . . . . . . . . 24

15 The hexagonal drift cells of the DCs. The sense wires are located in the center of each cell, while the field wires are located in the vertices of the hexagons. $\ldots \ldots \ldots \ldots \ldots \ldots$

16 Schematic view of the CCs in one of the CLAS sectors. . . . . . . . . . . . . . . . . 26

17 A cherenkov counter with a typical electron track crossing the radiator gas producing photo-electrons,

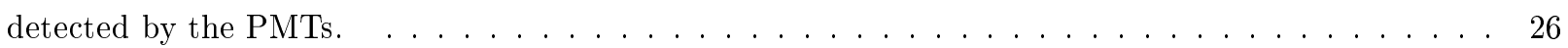

18 Schematic view of the TOF scintillators in one sector of CLAS. . . . . . . . . . . . . . . . 27

19 (a) U, V, and W orientations in an EC module. (b) hit reconstruction in the EC sectors. . . . . . . 28

20 The EG2 target $[22]$. The red square shows the $\mathrm{LD}_{2}$ target cell. The blue square shows the solid

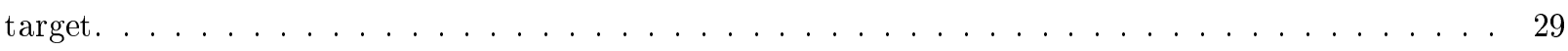

21 The EC fiducial region for electron candidates in the global $E C_{X}, E C_{Y}$ coordinates, where $E C_{Z}$ is

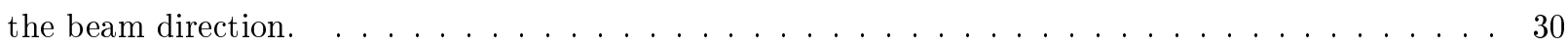

22 Distribution of the number of photo-electrons $\left(N_{\text {photo electrons }}\right)$ produced in CC by electron candidates

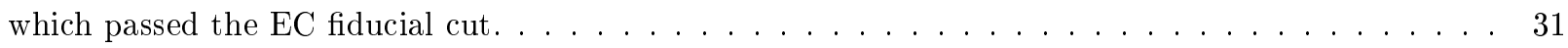

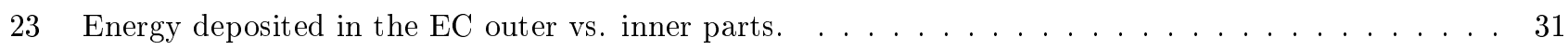

24 Momentum-normalized energy deposited in the EC outer vs. inner parts. . . . . . . . . . . . 32

25 Corrected proton vertex TOF vs. momentum for a sample of proton-candidates' tracks. . . . . . . . 33

\begin{tabular}{|lll}
26 & $\Delta \beta$ vs. momentum for a chosen sample of positively (left) and negatively (right) charged particles. 34 \\
\hline
\end{tabular}

$27 \quad$ The corrected electron (left) and proton (right) target vertex distributions for each CLAS sector. . 34

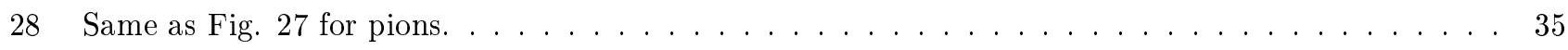

29 The maximum absolute difference between all charged particles vertex positions shown in units of $\sigma$, 36 
30 The $d\left(e, e^{\prime} p \pi^{+} \pi^{-}\right) X$ missing mass distribution. The red lines show the cut that is applied to select

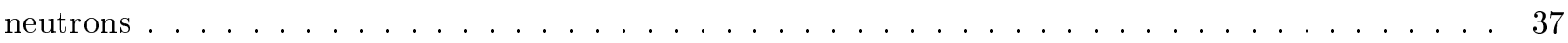

31 The missing momentum distribution. The red line shows the applied $P_{m i s s}>0.5 \mathrm{GeV} / \mathrm{c}$ cut. . . . . 37

32 The cosine of the angle between the EC hit location and the missing momentum direction distribution for $d\left(e, e^{\prime} p \pi^{+} \pi^{-}\right) n$ events that have a neutral hit in the EC, . . . . . . . . . . . . . . . . . 38

33 Left: The correlation between the missing momentum and the reconstructed neutron momenta before applying any corrections to the reconstructed momenta. . . . . . . . . . . . . . . . . . . . . . 39

34 The EC momentum resolution (the width of the $\Delta p / p$ distribution) as a function of the measured neutron momentum. . . . . . . . . . . . . . . . . . . . . . . . . 39

35 The neutron detection efficiency as a function of the momentum for each sector (points) together with a two-parameter fit to the efficiency for each sector . . . . . . . . . . . . . . . . . 40

36 The missing mass distributions along with their fits for $d\left(e, e^{\prime} p \pi^{+} \pi^{-}\right) n$ events (left) and $d\left(e, e^{\prime} p \pi^{+} \pi^{-} n\right)$ events (right) . . . . . . . . . . . . . . . . . . . . . . . . . 4 41

37 The difference between the original proton momentum $\left(p_{p}\right)$ and the smeared proton momentum $\left(p_{\text {smeared }}\right)$. . . . . . . . . . . . . . . . . . . . . . . . . . . . 4 42

$38 \quad Q^{2}$ normalized distributions for: (a) un-smeared protons events with $x_{B}>1.2$, and (b) smeared protons (red) and neutrons (blue) . . . . . . . . . . . . . . . . . . . . . . . . 44

$39 \quad \theta_{N q}$ vs. $\left|\vec{p}_{N}\right| /|\vec{q}|$ for un-smeared protons. The red box shows the cut applied to select leading protons in $[6] . \ldots \ldots \ldots \ldots \ldots \ldots \ldots$

40 Same as Fig. 39 for smeared protons (a) and neutrons (b). . . . . . . . . . . . . . . . . . . . . . . 45

41 The false positive (left) and negative (right) probabilities, as a function of the smeared protons lower missing momentum cut for different cuts on the missing mass . . . . . . . . . . . . . . . . 46

42 The electron kinematic variables for smeared protons (red) and neutrons (blue) after applying the high missing momentum cuts. . . . . . . . . . . . . . . . . . . . . . . . 47

43 The smeared-proton (red) and the neutron (blue) kinematic variables after applying the high missing momentum cuts. . . . . . . . . . . . . . . . . . . . . . 48

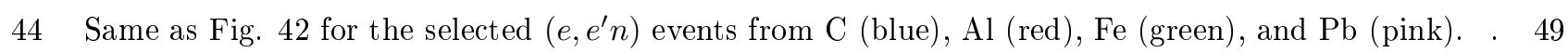

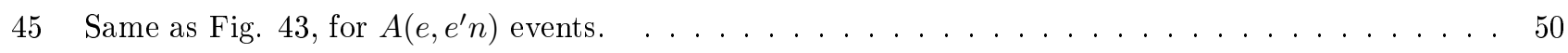

46 The missing energy vs. the missing momentum for (a) (e, $\left.e^{\prime} p\right)$ events, (b) (e, $\left.e^{\prime} n\right)$ events and (c)

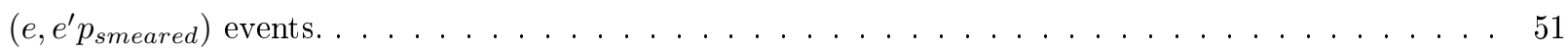

$47 \quad$ (a) The $y$ scaling variable vs. $\omega$ with (right) and without (left) the $p_{m i s s} \& E_{m i s s}$ QE cuts for

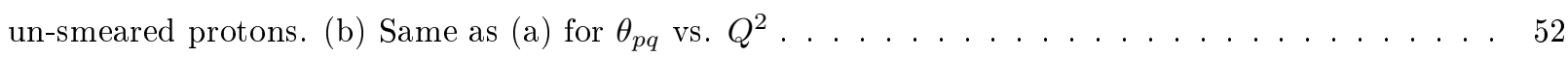

48 The false positive (left) and negative (right) probabilities for the low missing momentum (QE) events, as a function of the missing momentum cut for different missing energy cuts. . . . . . . . . . . . . . 53

49 The electron kinematic variables for ${ }^{12} \mathrm{C}\left(e, e^{\prime} p_{\text {smeared }}\right)(\mathrm{red})$ and ${ }^{12} \mathrm{C}\left(e, e^{\prime} n\right)$ (blue) after applying the

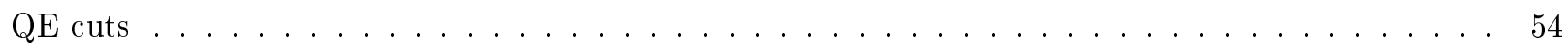

50 The smeared protons (red) and neutrons (blue) quantities after applying the QE cuts . . . . . . . . 55

$51 \quad$ Same as Fig. 49 for $A\left(e, e^{\prime} n\right)$ events after applying the QE cuts . . . . . . . . . . . . . . . . . . . . 56

52 Same as Fig. 50 for $A\left(e, e^{\prime} n\right)$ events after applying the QE cuts . . . . . . . . . . . . . . . . . . . . 57

53 The difference between the energy of generated and reconstructed $\left(E_{G e n}-E_{R e c}\right)$ protons as a function of the reconstructed proton momentum $\left(p_{R e c}\right)$, extracted using GSIM $[20 \mid$. . . . . . . . . . . . . . . 58

54 Energy deposited in the TOF counters for the recoil protons vs. their momentum for the selected smeared $\left(e, e^{\prime} p p\right)(\mathrm{red})$ and $\left(e, e^{\prime} n p\right)$ (blue) events. . . . . . . . . . . . . . . . . . . . . . 59

55 The electron kinematic variables for smeared ${ }^{12} \mathrm{C}\left(e, e^{\prime} p p\right)$ (red) and ${ }^{12} \mathrm{C}\left(e, e^{\prime} n p\right)$ (blue) selected events. 60

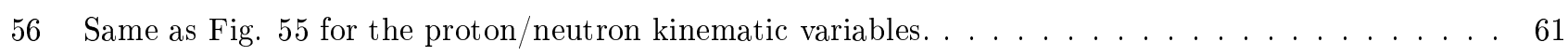


57 Same as Fig. $|55|$ for the recoil proton kinematic variables. . . . . . . . . . . . . . . . . . . 62

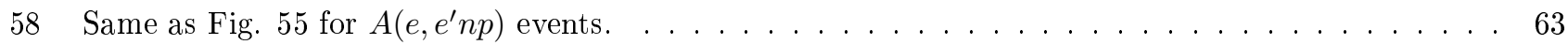

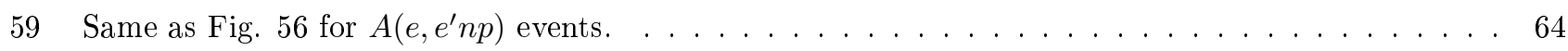

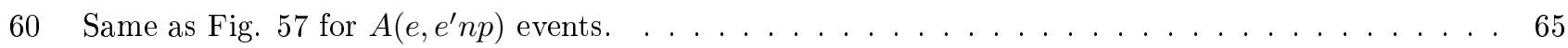

61 The $A$-dependence of the $A\left(e, e^{\prime} p\right) / C\left(e, e^{\prime} p\right)$ cross-section ratios for smeared protons (red), and un-

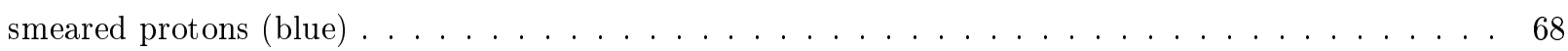

62 Same as Fig. 61 for the neutrons ratios. . . . . . . . . . . . . . . . . . . . . . . . . 69

63 Relative high-momentum fractions for protons (red circles) and neutrons (blue squares). The inner error bars are statistical and the outer ones include both statistical and systematic uncertainties. . . 70

64 The simulated corrections factor as a function of the scattering angle of the proton and its momentum. 73

65 Relative abundances of high- and low-initial-momentum neutrons and protons. Reduced cross-section ratio, $\left[\sigma_{A}\left(e, e^{\prime} n\right) / \sigma_{n}\right] /\left[\sigma_{A}\left(e, e^{\prime} p\right) / \sigma_{p}\right]$, for low-momentum and high-momentum events. $\ldots \ldots \ldots$. . 75

66 Extracted ratios of $p p$ - to $n p$-SRC pairs in nuclei. The open symbols show the measured reduced cross-section ratios $R=\left[A\left(e, e^{\prime} p p\right) / 2 \cdot \sigma_{p}\right] /\left[A\left(e, e^{\prime} n p\right) / \sigma_{n}\right] \ldots \ldots \ldots \ldots \ldots \ldots \ldots$

67 The extracted transparency ratios for MF and SRC kinematics, both for protons and neutrons, as a

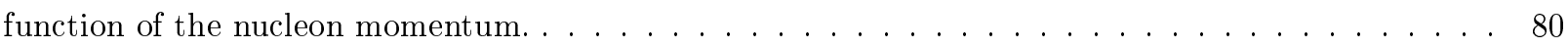

68 The extracted transparency ratios for MF and SRC kinematics, both for protons and neutrons, together with a power law fit to a weighted average (grey line) $\ldots \ldots \ldots \ldots 1$

$69 \quad$ Relative high-momentum fractions for protons (red circles) and neutrons (blue squares). . . . . . . 83

70 Relative abundances of high- and low-initial momentum neutrons and protons. Reduced cross-section ratio, $\left[\sigma_{A}\left(e, e^{\prime} n\right) / \sigma_{n}\right] /\left[\sigma_{A}\left(e, e^{\prime} p\right) / \sigma_{p}\right]$, for low-momentum and high-momentum events. $\ldots \ldots$. . 83

71 Extracted ratios of $p p$ - to $n p$-SRC pairs in nuclei. The open symbols show the measured reduced

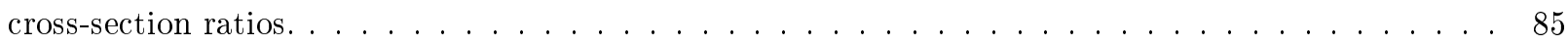

72 The false positive (left) and negative (right) probabilities for ${ }^{27} \mathrm{Al} . \ldots \ldots \ldots \ldots$

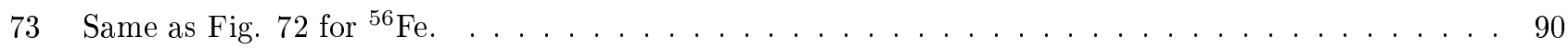

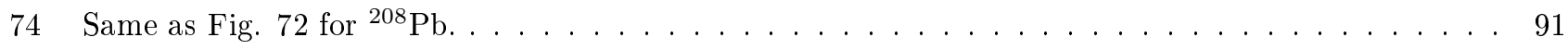

75 Electron kinematics variables for the selected $\left(e, e^{\prime} p_{\text {smeared }}\right)$ events from ${ }^{12} \mathrm{C}$ (blue) $,{ }^{27} \mathrm{Al}(\mathrm{red}),{ }^{56} \mathrm{Fe}$

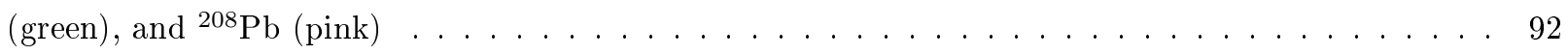

$76 \quad$ Smeared protons kinematics for the selected $\left(e, e^{\prime} p_{\text {smeared }}\right)$ events from ${ }^{12} \mathrm{C}$ (blue), ${ }^{27} \mathrm{Al}(\mathrm{red}),{ }^{56} \mathrm{Fe}$

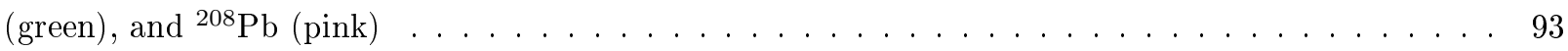

77 The missing energy vs. the missing momentum for (a) ${ }^{27} \mathrm{Al}\left(e, e^{\prime} p\right)$ data, (b) ${ }^{56} \mathrm{Fe}\left(e, e^{\prime} n\right)$ data, and (c) ${ }^{208} \mathrm{~Pb}\left(e, e^{\prime} p_{\text {smeared }}\right)$ data $\ldots \ldots \ldots \ldots \ldots \ldots \ldots \ldots \ldots$

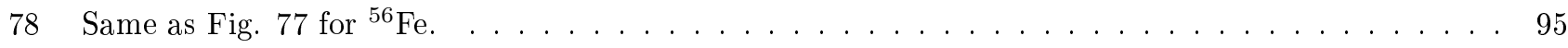

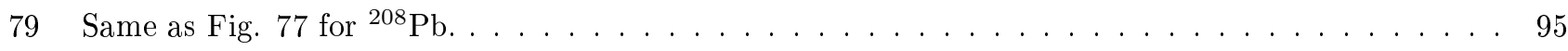

$80 \quad{ }^{27} \mathrm{Al}:$ (a) The $y$ scaling variable vs. $\omega$ with (right) and without (left) the QE cuts for un-smeared protons. (b) Same as (a) for $\theta_{p q}$ vs. $Q^{2} . \ldots \ldots \ldots \ldots \ldots \ldots \ldots$

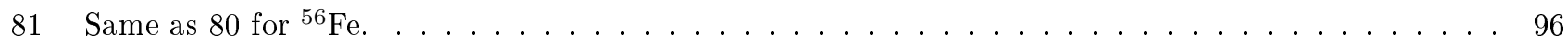

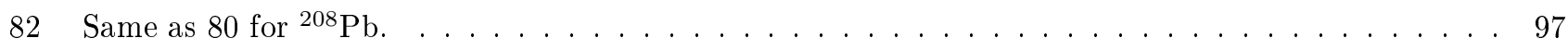

83 The false positive (left) and negative (right) probabilities for the low missing momentum (QE) events, as a function of the missing momentum cut for different missing energy cuts, for ${ }^{27} \mathrm{Al}$. . . . . . . . 97

84 Same as Fig. $83 \mid$ for ${ }^{56} \mathrm{Fe} . \ldots \ldots \ldots \ldots \ldots \ldots \ldots \ldots \ldots$

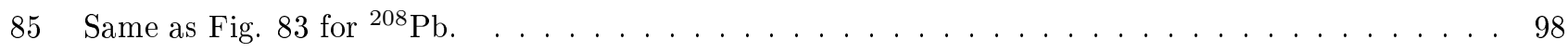

86 The electron kinematic variables for ${ }^{12} \mathrm{Al}\left(e, e^{\prime} p_{\text {smeared }}\right)$ (red) and ${ }^{12} \mathrm{Al}\left(e, e^{\prime} n\right)$ (blue) after applying the QE cuts: (a) the electron momentum, (b) the electron scattering angle, (c) $Q^{2}$, (d) $\omega$, (e) $y$, and

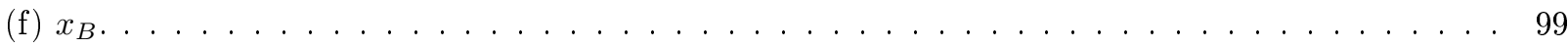


87 Same as Fig. $86 \mid$ for ${ }^{56} \mathrm{Fe}$. . . . . . . . . . . . . . . . . . . . . . . . . 100

88 Same as Fig. 86 for ${ }^{208} \mathrm{~Pb}$. . . . . . . . . . . . . . . . . . . . . . . . . . . . . 101

89 The smeared protons (red) and neutrons (blue) quantities after applying the QE cuts for ${ }^{27} \mathrm{Al}:$. . . 102

90 Same as Fig. 89 for ${ }^{56} \mathrm{Fe}$. . . . . . . . . . . . . . . . . . . . . . . . . . 102

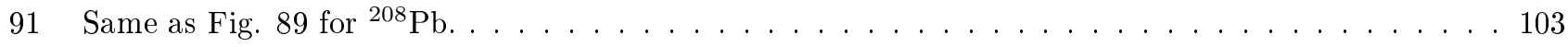

92 The electron kinematic variables for smeared $A\left(e, e^{\prime} p p\right)$ events. Blue - C, yellow - Al, red - Fe, and green - Pb. Shown are the electron momentum, the electron scattering angle, $Q^{2}, x_{B}$, and $\omega$. . . . . 104

93 he proton kinematic variables for smeared $A\left(e, e^{\prime} p p\right)$ events. Blue - C, yellow - Al, red - Fe, and green - Pb. Shown are the proton momentum, the proton scattering angle, and the angle . . . . . . . . 105

94 The recoil proton kinematic variables for smeared $A\left(e, e^{\prime} p p\right)$ events. Blue - C, yellow - $\mathrm{Al}, \mathrm{red}-\mathrm{Fe}$, and green - Pb. Shown are its momentum, its scattering angle . . . . . . . . . . . . . . . 106

95 The PDF distribution of the $p p / n p$ ratio. The solid red line marks the median value . . . . . . . . . 108 


\section{List of Tables}

1 The proton and neutron average kinetic energies as extracted from VMC single-nucleon momentum distribution calculations $[37 \mid . \ldots \ldots \ldots \ldots \ldots \ldots$

$2 \quad$ The proton and neutron average kinetic energies calculated using the $n p$-dominance model. . . . . . 20

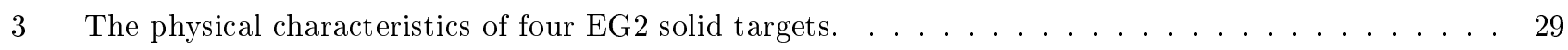

$4 \quad$ Values for the energy shifts due to coulomb distortions for each target nuclei. . . . . . . . . . . . 33

5 The fit parameters for the momentum dependence of the neutron detection efficiency and the efficiency

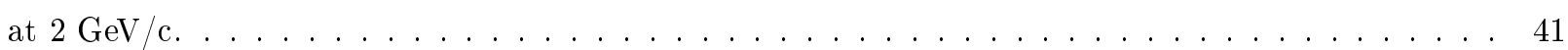

$6 \quad$ The signal-to-background ratios for different missing momentum bins, and their corrected efficiencies. 42

7 The selected cuts of this analysis $\left(1^{\text {st }}\right.$ column), and the cuts used in the knockout proton analysis $|6|$ $\left(2^{n d}\right.$ column $) \ldots \ldots \ldots \ldots \ldots \ldots \ldots \ldots$

8 The false positive and negative probabilities of all targets for low-momentum (left) and high-momentum

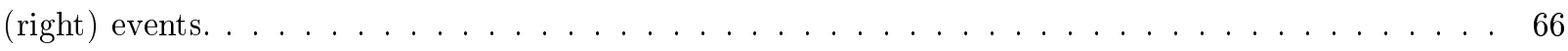

$9 \quad$ The M.F. event-selection cuts, and the range used for the sensitivity study. . . . . . . . . . . . 67

$10 \quad$ The high-momentum event-selection cuts, and the range used for the sensitivity study. . . . . . . . . 67

11 The event-selection cuts, and the change in the ratios due to variations in the cuts, for smeared

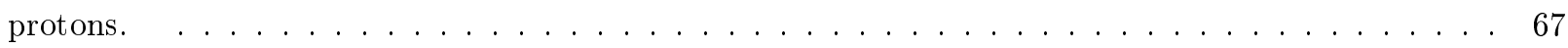

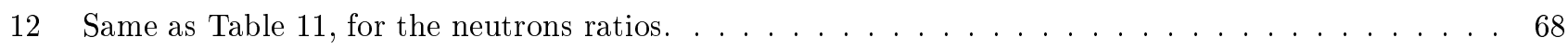

13 The measured double-ratios and their total uncertainties shown in Fig. 63 . . . . . . . . . . . . . 70

14 The event selection cuts, and the change in the ratios due to variations in the cuts, for $A\left(e, e^{\prime} n\right) / A\left(e, e^{\prime} p\right)$

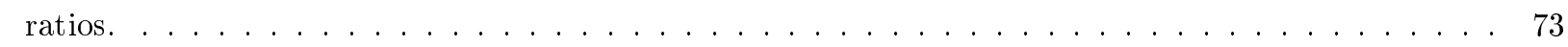

$15 \quad \frac{A\left(e, e^{\prime} n\right)}{A\left(e, e^{\prime} p\right)}$ M.F. ratios and the different contributions to the uncertainty. . . . . . . . . . . . . 74

$16 \quad \frac{A\left(e, e^{\prime} n\right)}{A\left(e, e^{\prime} p\right)} \mathrm{SRC}$ ratios and the different contributions to the uncertainty. . . . . . . . . . . . . . 74

17 The $\left(e, e^{\prime} N p\right)$ event selection cuts. Also shown is the sensitivity of the $p p / n p$ ratios to variations of

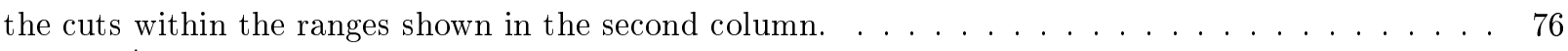

18 The $\frac{A\left(e, e^{\prime} p p\right) / 2 \cdot \sigma_{p}}{A\left(e, e^{\prime} n p\right) / \sigma_{n}}$ M.F. ratios and the different contributions to the uncertainty $\ldots \ldots \ldots \ldots 77$

$19 \quad$ Measured reduced cross-section $R$ ratios and extracted $\# p p-S R C / \# n p-S R C$ pairs ratio. . . . 78

20 Left - The transparency ratios and their uncertainties for the MF kinematics. For each nucleus, the left and the right values are for proton and neutron knockout respectively $\ldots \ldots \ldots \ldots$. . . . . 81

21 The SCX probabilities for different $N N$ pairs and nuclei. $\ldots \ldots \ldots \ldots \ldots$. . . . . . 108 


\section{Introduction}

The atomic nucleus is composed of nucleons: positively charged protons and neutral neutrons. In 1932, Chadwich discovered the neutron [1] paving the way to the modern picture of the atomic nucleus. A main goal of nuclear physics reaserch was, and still is, understanding the nature of the interaction between nucleons, $N N$-interaction, and how this interaction makes up the atomic nuclei and determines their properties. The fact that we have stable nuclei means that the repulsive electromagnetic interaction is weaker than the strong $N N$-interaction, which is attractive at distances of a few fermi and repulsive at shorter distances.

The most general non-relativistic Hamiltonian for nuclei with $A$ nucleons, neglecting possible contributions from non-nucleonic degrees of freedom, is given by:

$$
H=T+\sum_{i<j}^{A} \nu_{2 b o d y}(i, j)+\sum_{i<j<k}^{A} \nu_{3 b o d y}(i, j, k)+\ldots
$$

where $T$ is the kinetic energy and $\nu_{2 b o d y}$ and $\nu_{3 b o d y}$ are the two and three nucleon potentials.

The nuclear shell model was the first successful effective description of atomic nuclei [2]. This model predicts successfully many properties of nuclei such as spins, parities, ground-state energies, excitation spectra, and others. In this approximate model, the nucleus is a collection of individual independent nucleons that move under the influence of a mean-field potential created by all the other $(A-1)$ nucleons. Protons and neutrons are moving independently in well defined shell model states. The quantum shells are populated sequentially according to the Pauli exclusion priniciple, and nucleon transitions are allowed only to non occupied shells. Under this approximation we can divide Eq. 1 into two parts, the shell model part:

$$
H_{\text {shell }}=T+\sum_{i=1}^{A} V_{\text {mean-field }}(i)
$$

and the residual interaction part:

$$
H_{\text {res }}=\left[\sum_{i<j}^{A} \nu_{2 b o d y}(i, j)+\sum_{i<j<k}^{A} \nu_{3 b o d y}(i, j, k)+\ldots\right]-\sum_{i=1}^{A} V_{\text {mean-field }}(i)
$$

where $V_{\text {mean-field }}$ is the effective mean field potential described above. In the mean-field approximation the residual many-body interaction $H_{\text {res }}$ is assumed to vanish.

Electron-scattering proton-knockout measurements $A\left(e, e^{\prime} p\right)$ in the early 1980s [3] showed that the energy and momentum distributions of bound protons matched shell-model predictions, which was a great success for the model. However, when looking at the cross-sections for valence proton knockout compared to these predicted by the model, only about 60 - $70 \%$ of the expected strength is observed (Fig. 1) 4. The 30-40\% "missing strength" observed in these experiments is attributed to correlations between the nucleons, which are not taken into account in an independent particle or shell-model mean-field approach. Such nucleon-nucleon (NN) correlations are often characterized as long-range correlations (LRC) and short-range correlations (SRC), referring to their spatial 
separation and the relevant part of the NN potential. The SRC correlations discussed below account for about half of the missing strength.

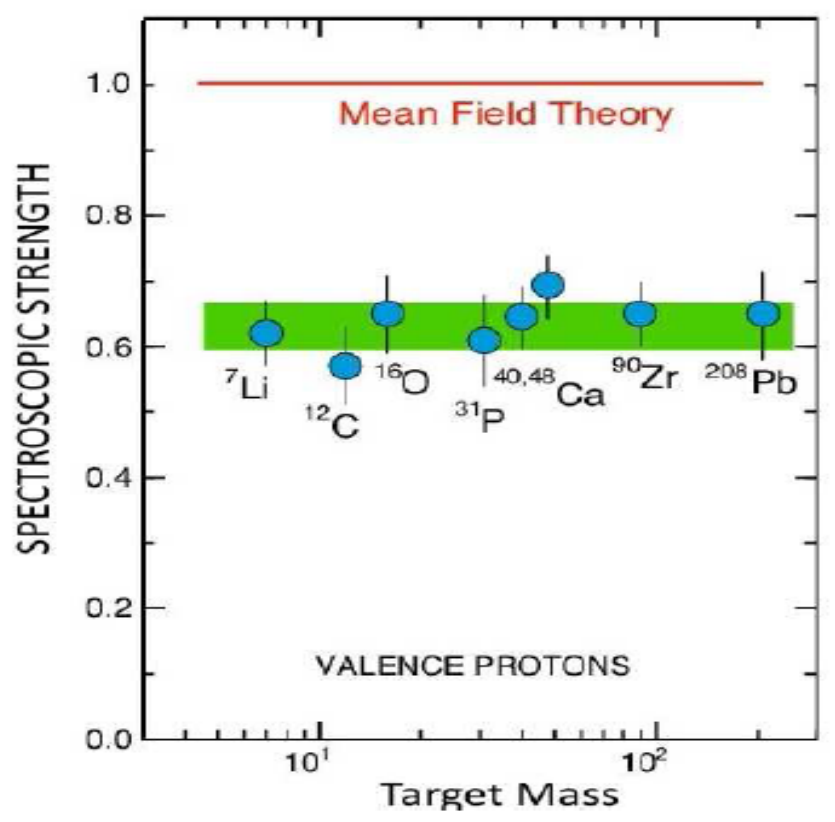

Figure 1: The spectroscopic strength for various nuclei, extracted by comparing $A\left(e, e^{\prime} p\right)$ valence knockout crosssections to mean-field calculations. Figure adapted from 4 .

\section{$1.1 \quad 2 \mathrm{~N}-\mathrm{SRC}$}

The typical separation between nucleons in the nucleus is $\sim 1.7 \mathrm{fm}$. However, as the nucleons are moving, they sometimes form short-lived correlated nucleon pairs at shorter distances. Due to the strong repulsion of the nucleon-nucleon force, the distance between two nucleons can not be too small. We consider a pair of $2 \mathrm{~N}-\mathrm{SRC}$, as close nucleons with a typical distance of $\sim 1 \mathrm{fm}$ between their centers, such that their wave-functions strongly overlap. In the momentum space we define them as a pair of nucleons with a small total (or c.m.) momentum $\left(\vec{p}_{c . m .}=\vec{p}_{1}+\vec{p}_{2}\right)$ and large relative momentum $\left(\vec{p}_{\text {rel }}=\left(\vec{p}_{1}-\vec{p}_{2}\right) / 2\right)$, where "small" and "large" are relative to the Fermi momentum of the nucleus $\left(k_{F}\right)$, which has a typical value of $\sim 250 \mathrm{MeV} / \mathrm{c}$ in medium and heavy nuclei.

Calculations of the single-nucleon momentum distributions in nuclei using independent particle shell models show a sharp decrease above the Fermi momentum. However, realistic many-body calculations, which include correlations between nucleons, show that the momentum distributions are extended to much larger momentum values, creating a "high-momentum tail". Figure 2 shows for ${ }^{12} \mathrm{C}$ the two types of expected momentum distributions discussed above.

The experimental study of SRC pairs was limited for many years. Experiments were done at several facilities trying to probe these pairs, however, due to the use of low energy probes, the SRCs were screened by other competing processes. With advances in accelerator technology, continous high intensity, high-momentum proton and electron beams became available, and the identification of SRCs in Quasi-Elastic (QE) scattering off nuclei became feasible. In this section, we review results from measurements of inclusive QE $\left(e, e^{\prime}\right)$ cross-section ratios, double-coincidence 
$\left(e, e^{\prime} p\right)$ and $(p, 2 p)$, and triple-coincidence $\left(e, e^{\prime} p N\right)$ and $(p, 2 p n)$, large momentum transfer (hard) reactions, performed at Stanford Linear Accelerator (SLAC), Brookhaven National Lab (BNL), and Jefferson Lab (JLab).

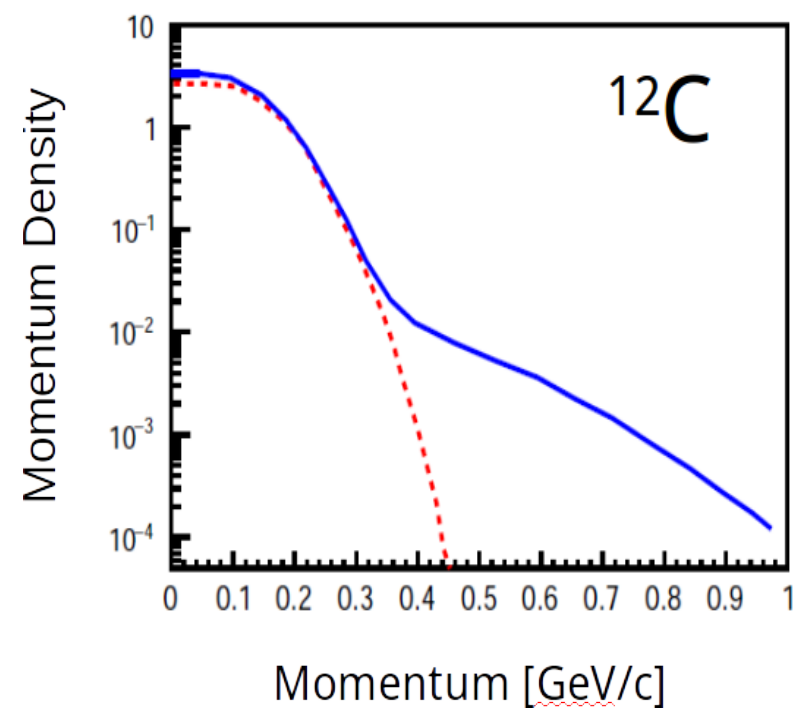

Figure 2: Calculation of the expected single-nucleon momentum distributions in ${ }^{12} \mathrm{C}$ based on the independent particle model (dashed red line), and with correlations (solid blue line). See [19] for more details.

\subsubsection{Quasi-Elastic (QE) Scattering}

In electron-nucleon scattering experiments, an electron beam with an incident energy $E$ scattering off a free nucleon yields electrons with an energy spectrum $E^{\prime}$ (i.e., energy-transfer of $\omega \equiv E-E^{\prime}$ ), at a fixed scattering angle. As can be seen in Fig. 3(a), the lowest energy transfer peak corresponds to elastic scattering, followed by the resonance region, beyond the pion production threshold. At larger energy-trasfer the reaction is dominated by deep inelastic scattering (DIS).

When scattering off a nucleus, the energy transfer spectrum becomes more complicated (see Fig. 3 (b)). The lowest energy transfer peak corresponds to elastic scattering off the nucleus as a whole, followed by exciting the residual nucleus to specific states. The wide peak corresponding to energy transfer around $\frac{Q^{2}}{2 M_{N}}$, where $Q^{2}$ is the four-momentum transfer of the reaction and $M_{N}$ the nucleon mass, can be identified as scattering off a single nucleon within the nucleus, marked in the figure as the QE peak. The peak is slightly shifted towards higher values of energy transfer with respect to the pure elastic scattering off a free nucleon (Fig. 3 (a)). This is due to the energy needed to remove the bound nucleon from the nucleus. The width of the QE peak is determined by the Fermi momentum of the nucleon in the nucleus. 

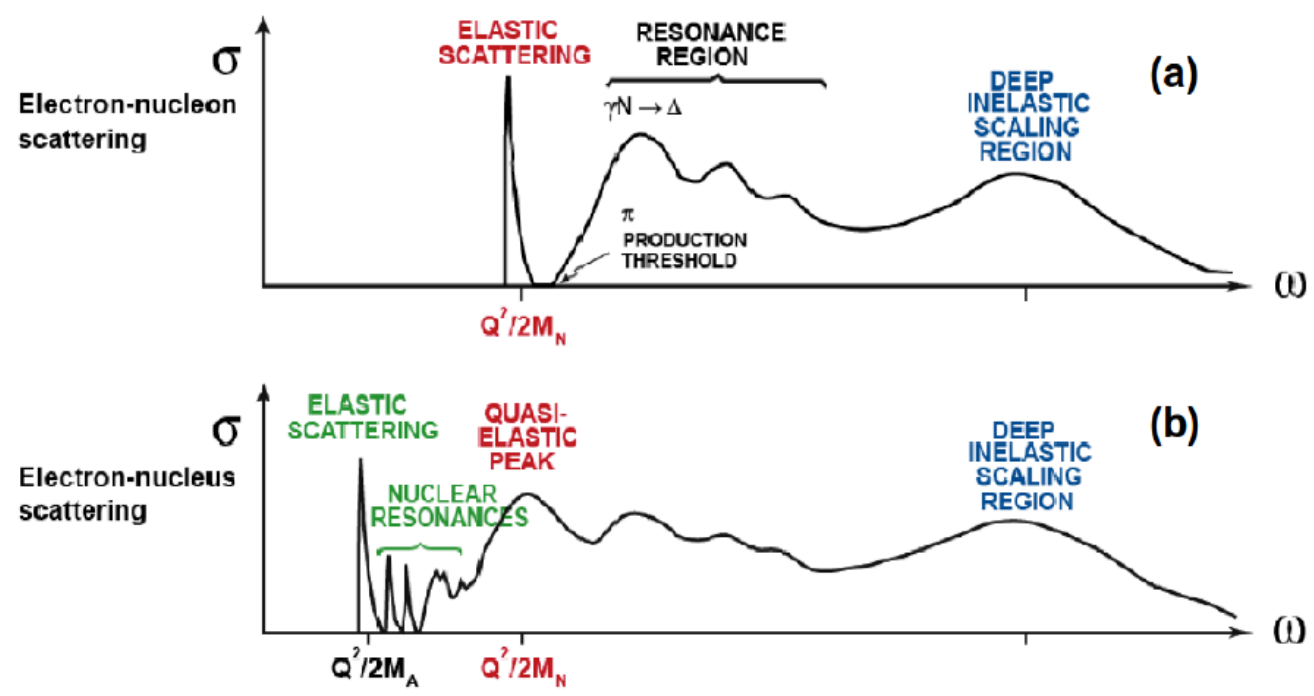

Figure 3: The electron-nucleon (a) and the electron-nucleus (b) cross-sections represented as a function of the energy transfer in the reaction.

\subsubsection{Inclusive SRC measurements}

In inclusive $\mathrm{QE} A\left(e, e^{\prime}\right)$ electron scattering off particles, two independent kinematical values are required to describe the reaction kinematically. Occasionally these are chosen to be $Q^{2}$ and $x_{B}=$ $\frac{Q^{2}}{2 M_{N} \omega}$. In the Plane-Wave Impulse Approximation (PWIA) it is assumed that the virtual photon is fully absorbed on a single nucleon, which is knocked-out from the nucleus without rescattering, leaving the remaining $A-1$ nuclear system unperturbed. The momentum of the $A-1$ system can be obtained from energy and momentum conservation:

$$
\left(q+p_{A}-p_{A-1}\right)^{2}=p_{f}^{2}=M_{N}^{2}
$$

where $q, p_{A}, p_{A-1}$, and $p_{f}$ are the four-momenta of the virtual photon, target nucleus, residual $A-1$ system, and knocked-out nucleon, respectively. This equation defines a simple relation between the minimal momentum of the knocked-out nucleon $\left(p_{m}^{\min }\right)$ and $x_{B}$ at fixed $Q^{2}$. Following 51 this relation is presented graphically in Fig. 4. As can be seen, at $x_{B}=1$, for all $Q^{2}$ values, the minimum value of this momentum component equals zero. As one increases or decreases $x_{B}$ at fixed $Q^{2}$, its value increases.

At large values of $Q^{2}\left(\sim 2-4 \mathrm{GeV}^{2} / \mathrm{c}^{2}\right)$ and $x_{B} \geq 1.4-1.5$ (or $\left.\leq \sim 0.6\right)$, this minimum value is larger than the Fermi momentum $\left(k_{F}\right)$, and the reaction is dominated by scattering off high-momentum $\left(k>k_{F}\right)$ nucleons in the nucleus. At these $Q^{2}$ values and $x_{B}<1$, the virtual photon carries a large amount of energy compared to its momentum and the reaction, while sensitive to high-momentum nucleons, has also large contributions from inelastic processes. For the same $Q^{2}$ values and $x_{B}>1$, the virtual photon transfers a small amount of energy compared to its momentum, and inelastic processes are suppressed. In these kinematics the reaction is more directly sensitive to the highmomentum tail of the nuclear wave function 45, 46. In both cases, large values of $Q^{2}$ suppress competing contributions from meson-excahnge and other two nucleon currents. 


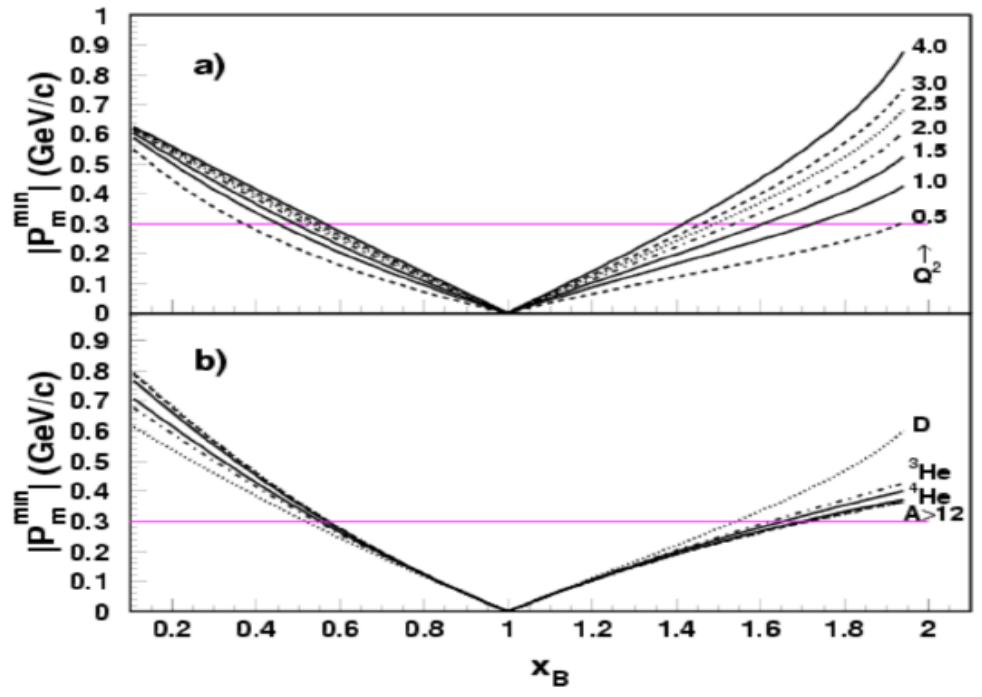

Figure 4: The minimum initial momentum of the knocked-out nucleon as a function of $x_{B}$. (a) For deuterium at different $Q^{2}$. (b) For different nuclei at $Q^{2}=2(\mathrm{GeV} / \mathrm{c})^{2}$. The horizontal lines represent an example of minimal momentum of $300 \mathrm{MeV} / \mathrm{c}$. The figure was adapted from Ref. [51.

Inclusive electron scattering cross-section ratios for nuclei $A$ relative to deuterium and to ${ }^{3} \mathrm{He}$ at high- $Q^{2}$ and $x_{B}>1$ were done at SLAC and JLab [51, 52, 53, 54, 32. Figure 5 shows the $x_{B^{-}}$ dependence of the per-nucleon cross-section ratio of nuclei relative to deuterium measured recntly at Hall-B [32. As can be seen, for $x_{B}$ values which correspond to scattering off high-momentum $\left(\geq k_{F}\right)$ nucleons in the nucleus (i.e., $\left.x_{B}>1.4\right)$, the cross-section ratio scales (i.e., does not depend on $\left.x_{B}\right)$.

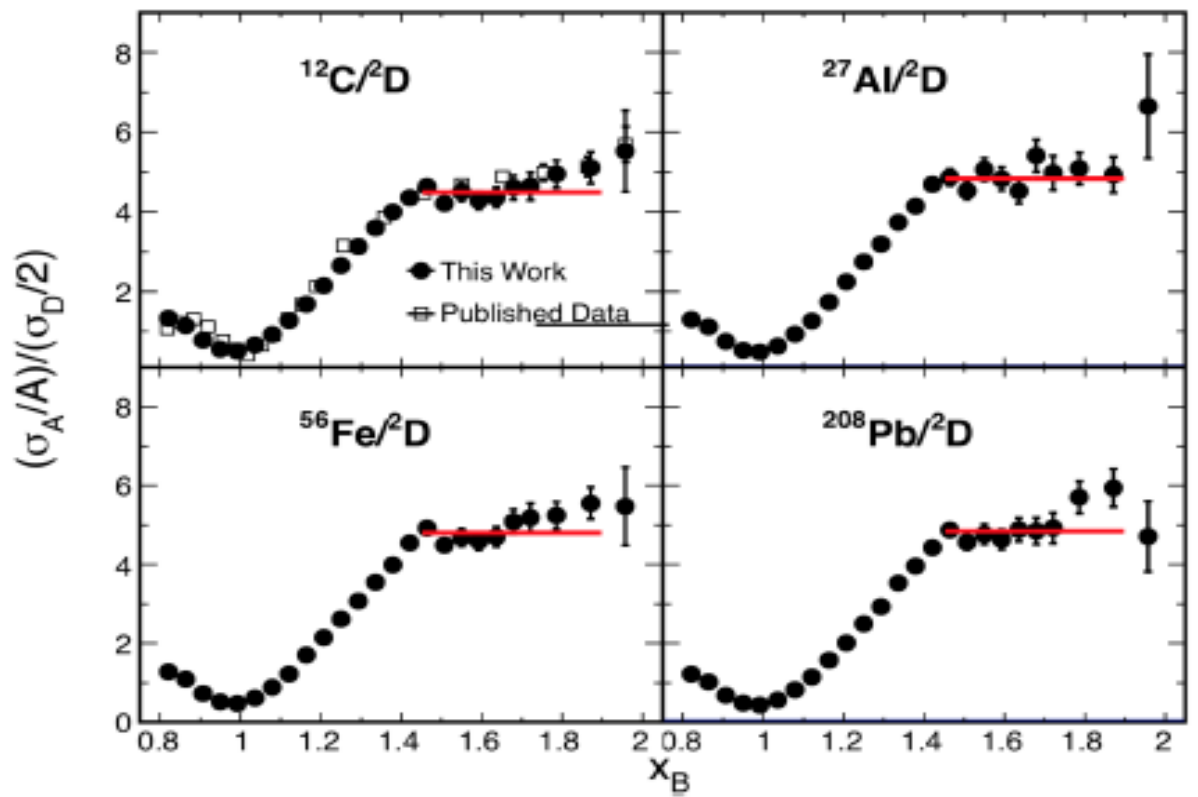

Figure 5: The per-nucleon QE $\left(e, e^{\prime}\right)$ cross-section ratios of nucleus $A$ to deuterium $\left(0.8<x_{B}<1.9\right.$, and $Q^{2}>1.5$ $\left.(\mathrm{GeV} / \mathrm{c})^{2}\right)$. The solid points show the data of 32 and the open squares the data of [54]. The red lines show a fit to a constant. The data are not isoscalar corrected (i.e., not corrected for the different electron-proton and electron-neutron elementary cross-sections). 
This scaling reflects the scaling of the high momentum tail of the nuclear wave function and was interpreted using the SRC model [52, 19]. The latter states that the high-momentum tail of the nuclear wave function is dominated by correlated, multi-nucleon, configurations. Due to their strong interaction at short distances, the structure of these configurations is independent of the surrounding nuclear environment, resulting in the same shape of the high-momentum tail in all nuclei (i.e., scaling), see Fig. 6. Different nuclei have different amounts of SRC clusters. In this model, the observed scaling of the per-nucleon cross-section ratios in $1.4<x_{B}<2$ is indicative of scattering off $2 \mathrm{~N}-\mathrm{SRC}$. The scaling factor, noted as $a_{2}(A / d)$ is a measure of the relative amount of $2 \mathrm{~N}-\mathrm{SRC}$ in the measured nuclei. In these unclusive measerements, the electrons can scatter off $p p, n n$ and $n p$ pairs, and therefore they do not give us information about the isospin structure of the SRC pairs.

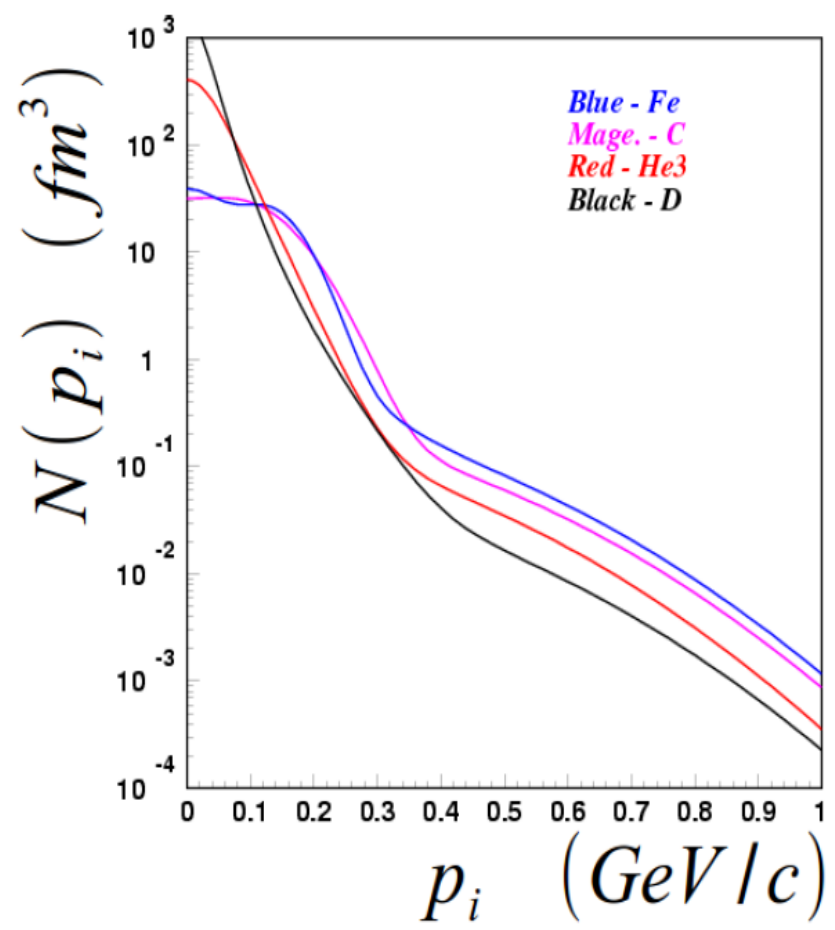

Figure 6: Calculated momentum distributions for different nuclei compared to deuterium [19. For low-momentum, the shape of the distributions very different for different nuclei. The high-momentum tail are similar in shape for all nuclei (i.e., scaling).

\subsubsection{Double- and Triple-coincidence measurements}

Inclusive measurements alone do not prove that high-momentum nucleons are a result of initialstate SRC pairs. To study the contribution of $2 \mathrm{~N}-\mathrm{SRC}$ pairs to the high-momentum tail of the nuclear wave function exclusive two-nucleon-knockout experiments were done. The idea is that, under the PWIA assumption, i.e., in the absence of FSI, if a nucleon that is part of a 2N-SRC pair is knocked out of the nucleus, in order to conserve momentum, its correlated partner nucleon has to recoil with momentum that is about equal in size and opposite in direction to the initial momentum of the knocked-out nucleon $\left(p_{\text {initial }}\right)$. This back-to-back correlation between the initial momentum of the knocked-out nucleon and the momentum of the recoil nucleon, both above $k_{F}$, is a clear signature off a $2 \mathrm{~N}-\mathrm{SRC}$ configuration. Due to c.m. motion of the pair with respect to the residual $A-2$ nuclear system, this correlation will not be exactly back-to-back. The measured 
angular correlation can be used to extract the c.m. momentum distribution of the pair. If the $2 \mathrm{~N}-\mathrm{SRC}$ model is correct, the nucleons in the pair will have large relative momentum $\left(>k_{F}\right)$ and small c.m. momentum $\left(<k_{F}\right)$, as discussed above.

The first two nucleon knockout experiments, measuring the ${ }^{12} \mathrm{C}(p, 2 p n)$ and ${ }^{12} \mathrm{C}\left(e, e^{\prime} p N\right)$ reactions, were done at BNL and JLab, respectively [55, 56, 57, 27]. These experiments scattered protons and electrons off high-initial momentum $\left(300 \leq p_{\text {initial }} \leq 600 \mathrm{MeV} / \mathrm{c}\right)$ protons in ${ }^{12} \mathrm{C}$ and looked for the emission of a correlated recoil nucleon. In the absence of FSI, the initial momentum of the struck nucleon equals the missing momentum of the ${ }^{12} \mathrm{C}(p, 2 p)$ and ${ }^{12} \mathrm{C}\left(e, e^{\prime} p\right)$ reactions. The BNL measurement was sensitive only to recoiling neutrons, while the JLab measurement was sensitive to both proton and neutron recoils. These experiments were performed at large momentum transfer $\left(Q^{2} \gtrsim 2(\mathrm{GeV} / \mathrm{c})^{2}\right.$ where competing effects are suppressed and FSI are mainly confined to be between the nucleons of the pair.

The main results of these experiments are shown in Figs. 7 and 8 . Figure 7 shows the distribution of the cosine of the opening angle between the initial momentum of the knocked-out proton and the recoil nucleon. The BNL results show a clear threshold around $k_{F}$ where recoiling neutrons above this momentum show a clear angular correlation, and those below it do not.
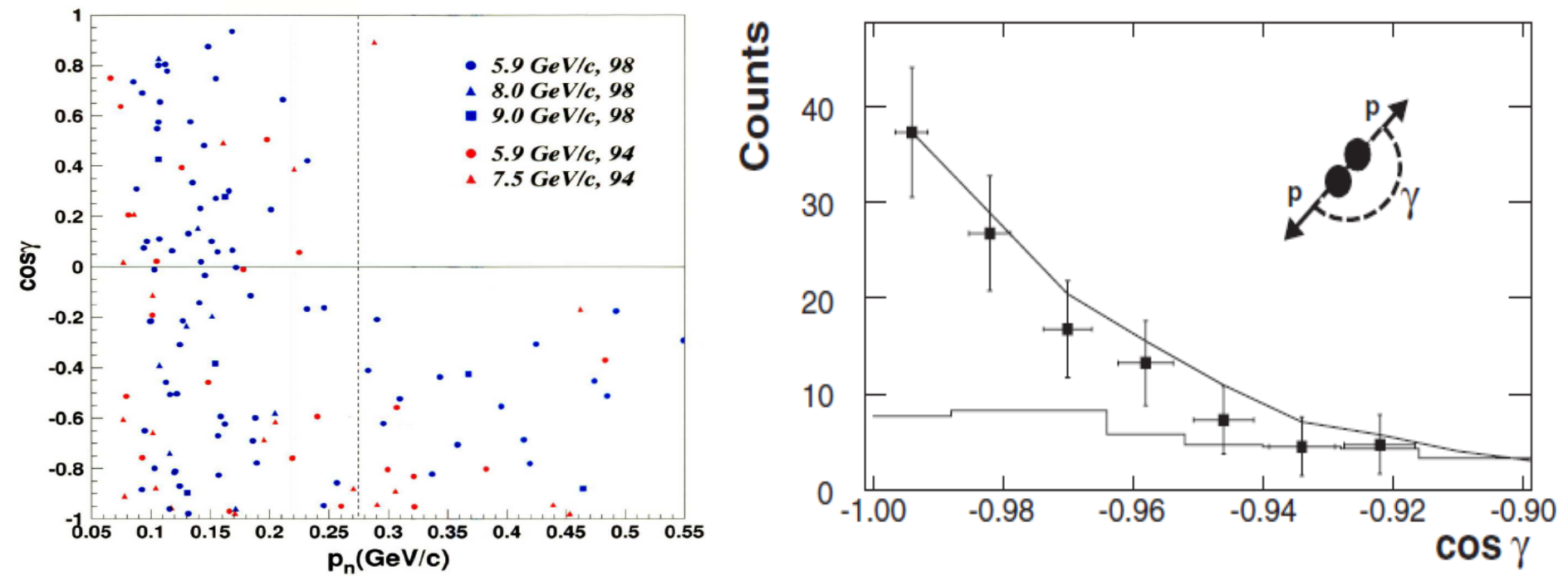

Figure 7: Distributions of cosine of the relative angle $(\gamma)$ between the reconstructed initial momentum of the knockout-proton and the recoil nucleon. Left: Results for ${ }^{12} \mathrm{C}(p, 2 p n)$ BNL measurements as a function of the momentum of the recoil neutron [56. Right: Results for ${ }^{12} \mathrm{C}\left(e, e^{\prime} p p\right)$ JLab measurements [57].

Figure 8 shows the ratio of single nucleon knockout events to two nucleon knockout events, corrected for finite acceptance effects, as a function of the missing momentum (the initial momentum of the knocked-out proton). As can be seen, within statistical uncertainties, all single proton knockout events were accompanied by the emission of a recoil nucleon. The ratio of proton recoil to neutron recoil was found to be approximately 1:20 [27]. These are clear evidences for the $2 \mathrm{~N}-\mathrm{SRC}$ dominance at the high-momentum tail and the importance of the tensor part of the nucleon-nucleon interaction at these momentum scales [58, 59]. 
The effect of these measurements on our understanding of the short distance nuclear structure is illustrated by the pie chart shown in Fig. 8. From inclusive measurements we know that in medium and heavy nuclei $(A \geq 12) \sim 80 \%$ of the nucleons are Mean-Field nucleons, whereas $\sim 20 \%$ have high-momentum. Combined with results from exclusive two-nucleon knockout measurements we know that these high-momentum nucleons are dominated by $2 \mathrm{~N}-\mathrm{SRC}$ pairs, and in particular pairs of neutron-proton.
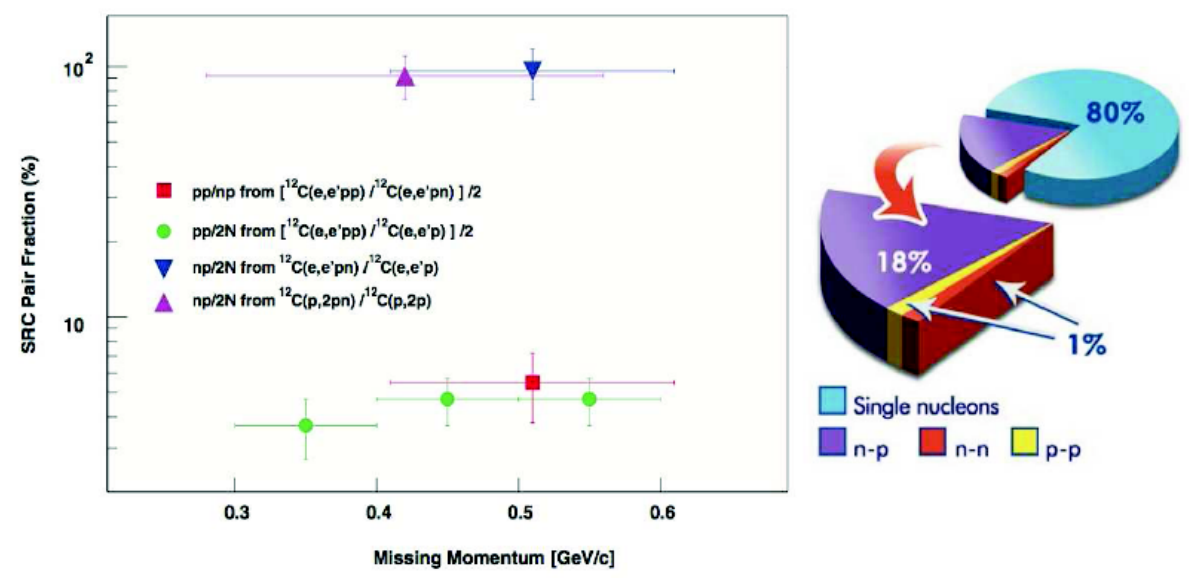

Figure 8: The ratio of ${ }^{12} \mathrm{C}\left(e, e^{\prime} p N\right)$ double knockout events to ${ }^{12} \mathrm{C}\left(e, e^{\prime} p\right)$ single knockout events as a function of the missing (initial) momentum of the knocked-out proton. Triangles and circles mark ${ }^{12} \mathrm{C}\left(e, e^{\prime} N n\right)$ and,${ }^{12} \mathrm{C}\left(e, e^{\prime} p p\right)$ events, respectively [27, 56. The square shows the ${ }^{12} \mathrm{C}\left(e, e^{\prime} p p\right) /{ }^{12} \mathrm{C}\left(e, e^{\prime} p n\right)$ ratio [27]. A clear dominance of $p n$ events is observed. The pie chart on the right illustrates our understanding of the structure of ${ }^{12} \mathrm{C}$, composed of $80 \%$ mean-field nucleons and $20 \%$ SRC pairs, where the latter is composed of $\sim 90 \% n p$-SRC pairs and $5 \% p p$ and nn SRC pairs each.

Another experiment measured the ${ }^{4} \mathrm{He}\left(e, e^{\prime} p p\right)$ and ${ }^{4} \mathrm{He}\left(e, e^{\prime} p n\right)$ reactions at JLab [49]. This experiment was done in a similar kinematics as in the previous experiments, and extended the proton missing (initial) momentum range up to $850 \mathrm{MeV} / \mathrm{c}$, to study the transition between the tensorforce to the repulsive hard core dominance.

Figure 9 shows the ratio of single-nucleon knockout events to two-nucleon knockout events, both for data corrected and un-corrected for FSI, as a function of the missing-momentum (the initial momentum of the knocked-out proton). Also shown is a comparision with theoretical calculations. As can be seen, the extracted fraction of proton-proton pairs is small at the measured missing momentum range. Figure 9 shows also the ratio between the fraction of $p p$ to $n p$ pairs. The dominance of $n p$ is clearly observed, but the abudance of $n p$ pairs is reduced as the missing momentum increases, due to the transition between SRC and the hard repulsive core. 


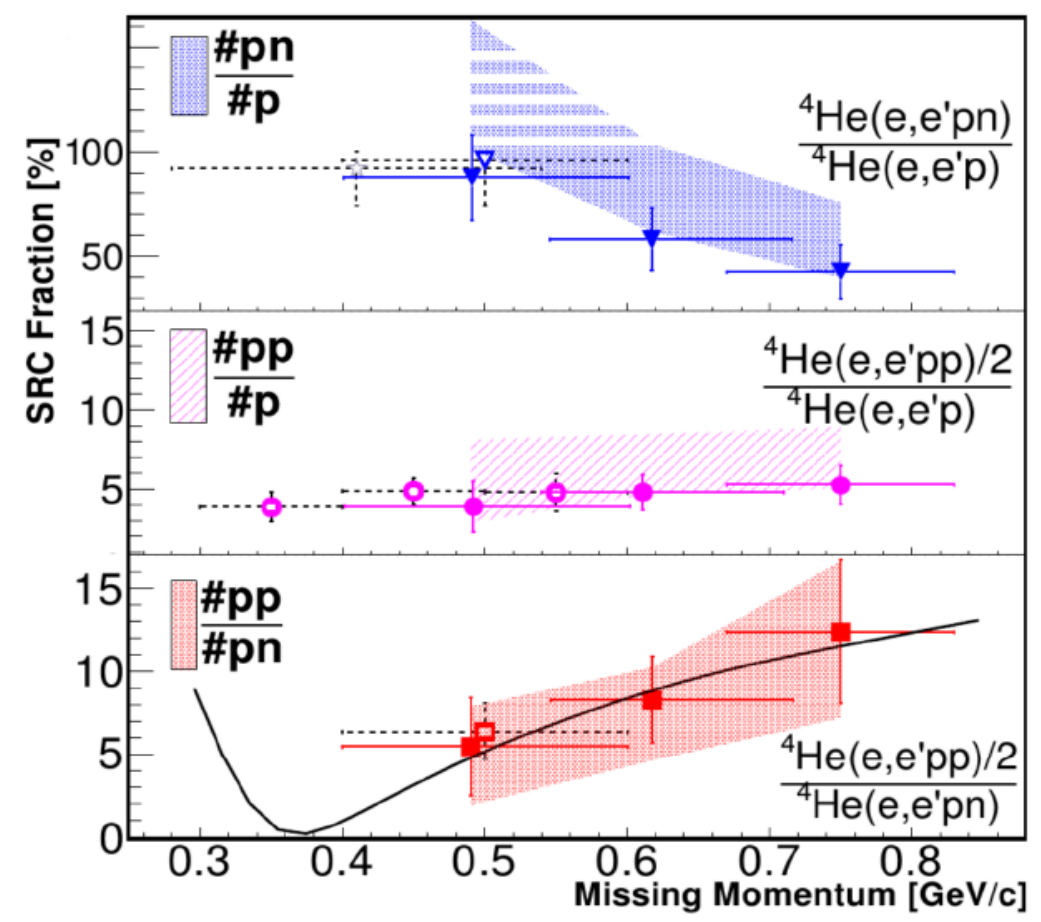

Figure 9: Top and middle panels: the ${ }^{4} \mathrm{He}\left(e, e^{\prime} p N\right) /{ }^{4} \mathrm{He}\left(e, e^{\prime} p\right)$ ratios, as a function of the knocked-out proton missing (initial) momentum. The bands represent the data corrected for FSI. Bottom panel: the ${ }^{4} \mathrm{He}\left(e,{ }^{\prime} p p\right) /{ }^{4} \mathrm{He}\left(e, e^{\prime} p n\right)$ ratios, together with a calculation [37] (solid black line).

A later study at JLab extend the previous measurements to heavy neutron-rich nuclei, by measuring the $\mathrm{A}\left(e, e^{\prime} p p\right)$ and $\mathrm{A}\left(e, e^{\prime} p\right)$ reactions, for ${ }^{12} \mathrm{C},{ }^{27} \mathrm{Al},{ }^{56} \mathrm{Fe}$, and ${ }^{208} \mathrm{~Pb}[6]$. The experiment was done in similar kinematics to the previous experiments, and with missing momentum between 300 and $600 \mathrm{MeV} / \mathrm{c}$. Figure 10 shows the extracted fractions of $n p$ and $p p$ SRC pairs from the sum of all SRC pairs in nuclei. The $n p$-dominance can be seen clearly in all measured nuclei, including neutron-rich imbalanced ones. The observed dominance of $n p$ over $p p$ pairs implies that even in heavy nuclei, SRC pairs are dominatly in a spin-triplet state, a consequence of the tensor part of the nucleon-nucleon interaction. It also implies that in neutron-rich there are as many highmomentum protons as neutrons, so the fraction of protons above the Fermi momentum is greater than that of neutrons in neutron-rich nuclei.

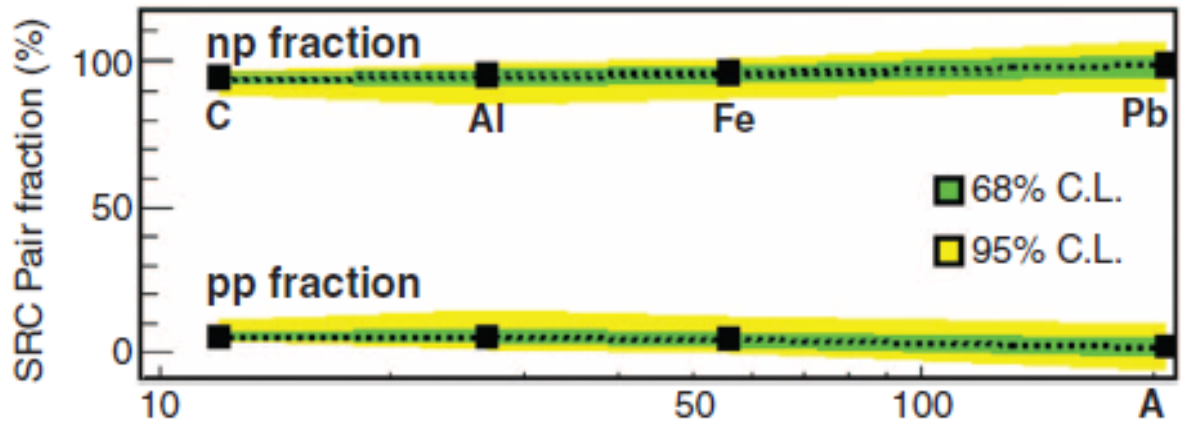

Figure 10: The extracted fractions of $n p$ (top) and $p p$ (bottom) SRC pairs from the sum of all SRC pairs in nuclei. The green and yellow bands reflect 68 and $95 \%$ confidence levels (CLs), respectively. $n p$-SRC pairs dominate over $p p$-SRC pairs in all measured nuclei 6 . 
The previous studies that showed the $n p$-dominance (i.e., the dominance of tensor force), using double and triple coincidence measurements, were all measurements with the knocked-out nucleon always being a proton. For light nuclei, ${ }^{4} \mathrm{He}$ and ${ }^{12} \mathrm{C}$, a recoil neutron was also detected in a coincidence with the proton. For the heavy neutron-rich nuclei, only protons were measured, both knocked-out and recoil. In this work we focus on these heavy neutron-rich nuclei. We report for the first time, measurements at which the knocked-out nucleon was a neutron. We do a simultaneous measurement of knocking out protons and neutrons, which allows us to compare and quantify the differences between protons and neutrons in neutron-rich nuclei. This is done using double and triple coincidence $A\left(e, e^{\prime} N\right)$ and $A\left(e, e^{\prime} N p\right)$ where $N=p / n$.

\subsection{Energy Sharing in Nuclear Systems}

In the case of light nuclei, Variational Monte-Carlo (VMC) calculations of the single-nucleon momentum distributions can be used to calculate the average kinetic energy of protons and neutrons 37. Based on these calculations, the average kinetic energy of protons in all bound neutron-rich light nuclei is larger than that of neutrons (see Table 1] 6]. However, such calculations do not exist for heavy nuclei, therefore we need to use effective models. One such model is the phenomenological $n p$-SRC dominance model 6, 38.

\begin{tabular}{|c|c|c|c|c|}
\hline Nucleus & Neutron Excess $[N / Z]$ & $\left\langle T_{p}\right\rangle[\mathrm{MeV}]$ & $\left\langle T_{n}\right\rangle[\mathrm{MeV}]$ & $\left\langle T_{n}\right\rangle /\left\langle T_{p}\right\rangle$ \\
\hline${ }^{8} \mathrm{He}$ & 3 & 30.13 & 18.60 & 0.62 \\
\hline${ }^{6} \mathrm{He}$ & 2 & 27.66 & 19.60 & 0.70 \\
\hline${ }^{9} \mathrm{Li}$ & 2 & 31.39 & 24.91 & 0.79 \\
\hline${ }^{3} \mathrm{H}$ & 2 & 19.61 & 14.96 & 0.76 \\
\hline${ }^{8} \mathrm{Li}$ & 1.67 & 28.95 & 23.98 & 0.83 \\
\hline${ }^{10} \mathrm{Be}$ & 1.50 & 30.20 & 25.95 & 0.86 \\
\hline${ }^{7} \mathrm{Li}$ & 1.33 & 26.88 & 24.54 & 0.92 \\
\hline${ }^{9} \mathrm{Be}$ & 1.25 & 29.82 & 27.09 & 0.91 \\
\hline${ }^{11} \mathrm{~B}$ & 1.20 & 33.40 & 31.75 & 0.95 \\
\hline${ }^{3} \mathrm{He}$ & 0.5 & 14.71 & 19.35 & 1.32 \\
\hline
\end{tabular}

Table 1: The proton and neutron average kinetic energies as extracted from VMC single-nucleon momentum distribution calculations [37. As can be seen, the average kinetic energy of the minority nucleons is larger than that of the majority nucleons.

This model describes the proton and neutron momentum distributions in all nuclei using a depleted mean-field approximation up to a transition momentum, which is near the nuclear Fermi sea level. Above that transition momentum we assume a deuterium-like high-momentum tail. The highmomentum tail is chosen to satisfy two constraints: 1) the fraction of high-momentum nucleons in nucleus $A$ relative to deuterium should equal $a_{2}(A / d)$, an experimental scaling factor extracted from inclusive $\left(e, e^{\prime}\right)$ cross-section measurements at $x_{B}>1.5$ [51, 53, 54, and 2) the absolute number of high-momentum protons and neutrons in a given nucleus should be equal, regardless of their overall relative abundance [38]. The resulting proton and neutron momentum distributions 
are given by 38 :

$$
n_{p}(k)=\left\{\begin{array}{cc}
\eta \cdot n_{p}^{M . F \cdot}(k) & k<k_{0} \\
\frac{A}{2 Z} \cdot a_{2}(A / d) \cdot n_{d}(k) & k>k_{0}
\end{array}\right.
$$

where $n_{p}^{M . F .}(k)$ is the mean-field proton momentum distribution in nucleus $A, n_{d}(k)$ is the deuterium momentum distribution. $a_{2}(A / d)$ is the experimentally measured per-nucleon probability of finding a high-momentum nucleon in nucleus $A$ relative to deuterium [51, 53, 54, 7], taken from column 6 of Table I in Ref. [7. $k_{0}$ is the transition momentum, and $\eta \leq 1$ is a normalization factor chosen such that $\int n_{p}(k) d^{3} k=1$. The neutron momentum distribution is obtained by replacing $n_{p}^{M . F .}(k)$ with $n_{n}^{M . F .}(k)$ and $Z$ with $N$ in Eq. 5 and re-normalizing.

The calculation was performed using three different models for the mean-field momentum distribution: Ciofi and Simula [19], Woods-Saxon [60], and Serot-Walecka [61]. $n_{d}(k)$ is calculated using the Argonne V18 NN potential [62 and $a_{2}(A / d)$ is taken from [7] based on inclusive $A\left(e, e^{\prime}\right)$ scattering measurements at $x_{B}>1.5[53$, 54]. Two sets of values of the transition momentum $k_{0}$ are used, (1) $k_{0}=300 \mathrm{MeV} / \mathrm{c}$ and (2) $k_{0}=k_{F}$, the Fermi momentum for nucleus A, where $k_{0}=221,260,260$, and $260(280) \mathrm{MeV} / \mathrm{c}$ for ${ }^{12} \mathrm{C},{ }^{27} \mathrm{Al},{ }^{56} \mathrm{Fe},{ }^{208} \mathrm{~Pb}$ for protons (neutrons) respectively [63]. The different proton and neutron Fermi momenta in ${ }^{208} \mathrm{~Pb}$ is due to its large neutron excess, which increases the average neutron density.

By integrating the momentum distributions of Eq. 5, the average proton and neutron kinetic energies in ${ }^{12} \mathrm{C},{ }^{27} \mathrm{Al},{ }^{56} \mathrm{Fe}$, and ${ }^{208} \mathrm{~Pb}$ were calculated. The resulting proton and neutron average kinetic energies for the mentioned nuclei are listed in Table 2. The range in the kinetic energies is due to the use of different models for the mean-field momentum distributions and different values for the transition momentum $k_{0}$.

\begin{tabular}{|c|c|c|c|c|}
\hline Nucleus & Neutron Excess $[N / Z]$ & $\left\langle T_{p}\right\rangle \mathrm{MeV}$ & $\left\langle T_{n}\right\rangle \mathrm{MeV}$ & $\left\langle T_{n}\right\rangle /\left\langle T_{p}\right\rangle$ \\
\hline${ }^{12} \mathrm{C}$ & 1 & $33.1-35.1$ & $33.1-35.1$ & 1.00 \\
\hline${ }^{27} \mathrm{Al}$ & 1.08 & $36.7-38.3$ & $36.3-36.9$ & $0.96-1.00$ \\
\hline${ }^{56} \mathrm{Fe}$ & 1.15 & $35.2-38.7$ & $32.5-36.0$ & $0.92-0.93$ \\
\hline${ }^{208} \mathrm{~Pb}$ & 1.54 & $41.7-42.4$ & $33.8-34.9$ & $0.80-0.82$ \\
\hline
\end{tabular}

Table 2: The proton and neutron average kinetic energies calculated using the $n p$-dominance model. The range in the kinetic energies is due to using different models for the mean-field momentum distributions and different values for the transition momenta $k_{0}$ as described in the text.

The results of the $n p$-SRC dominance model are also supported by a recent Low order Correlation operator Approximation (LCA) calculation [64. 


\section{Experimental Setup}

\subsection{The CEBAF Accelerator}

Jefferson Lab operates the Continuous Electron Beam Accelerator Facility (CEBAF), a superconducting high-current electron accelerator, that was designed to study nuclear and atomic physics. The maximum electron beam energy at the time of the experiment was $6 \mathrm{GeV}$, with momentum resolution of $<0.01 \%$, and a $100 \%$ duty cycle. Nowadays, after the JLab upgrade, the maximum energy is $12 \mathrm{GeV}$. A schematic layout of the accelerator is shown on Fig. 11. The electrons are pre-accelerated to $45 \mathrm{MeV}$ by the injector and injected into the accelerator from either a thermionic or a polarized electron gun. In the latter case, the source can supply up to $80 \%$ polarized electrons. After the injector, electrons are accelerated by the two main superconducting LINACs, which are connected by $180^{\circ}$ recirculation arcs. Each LINAC consists of three cryo modules, each contains eight Niobium cavities, which are kept superconducting at a temperature of approximately $2^{\circ} \mathrm{K}$. They are driven by a radio frequency $(\mathrm{RF})$ of $1.497 \mathrm{GHz}$. After passing through the second LINAC, the beam may be sent either to the experimental halls with energy of about $1.2 \mathrm{GeV}$, or, using the recirculating arcs, can be forwarded to the next acceleration cycle. Up to four recirculation (5passes) are possible. While the beam is in the accelerator, it is focused and steered with quadrapole and dipole magnets. The electron beam can be supplied to the three experimental Halls A, B and $\mathrm{C}$ simultaneously (after the JLab upgrade an additional experimental Hall-D began operation). The current and the beam energy can be controlled separately for each hall. The current delivered to Hall-B is limited by the maximum signal rates in the detector, and can achieve luminosity of $\sim 10^{34} \mathrm{~cm}^{-2} \mathrm{~s}^{-1}$ before losing its efficiency, Halls A and $\mathrm{C}$, can achieve $10^{38} \mathrm{~cm}^{-2} \mathrm{~s}^{-1}$.

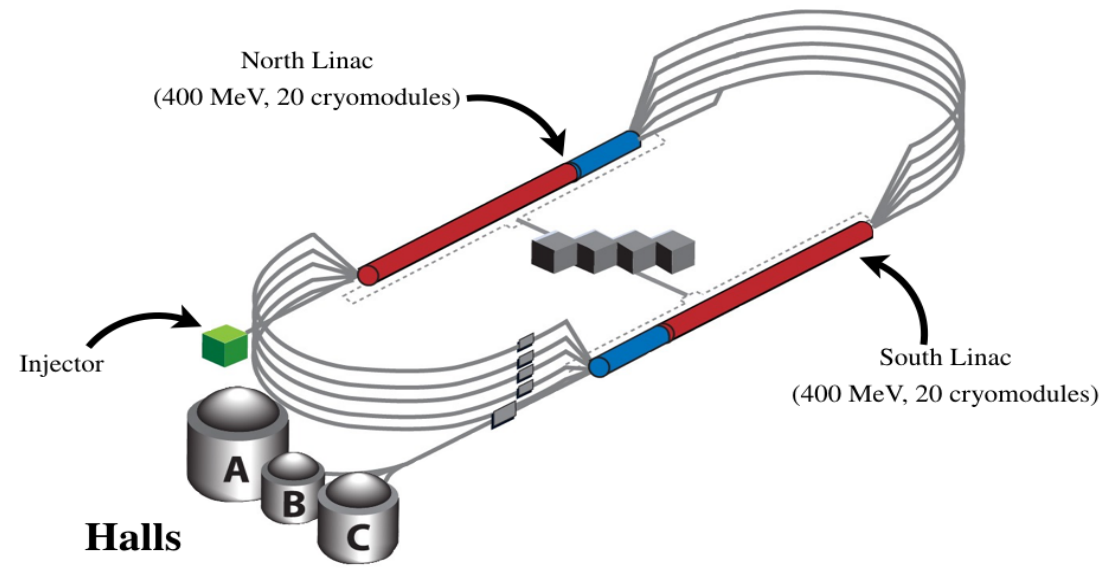

Figure 11: Schematic layout of JLab accelerator site (before the $12 \mathrm{GeV}$ upgrade).

\subsection{The CLAS Detector}

The CEBAF Large Acceptance Spectrometer (CLAS) is installed at Hall-B, to study multi-particle final state reactions. As can be seen in Fig. 12, CLAS has a large acceptance, of almost $4 \pi$. 
CLAS uses a toroidal magnetic field and six independent sectors of drift chambers, time-of-flight scintillation, Cherenkov counters, and electromagnetic calorimeters, covering scattering angles from about $8^{\circ}$ to $140^{\circ}$, for charged-particle identification and trajectory reconstruction. Because it requires different detector combinations, the coverage for neutral particles is about $8^{\circ}$ to $45^{\circ}$. Below, we describe in more details each of the subsystems of CLAS.

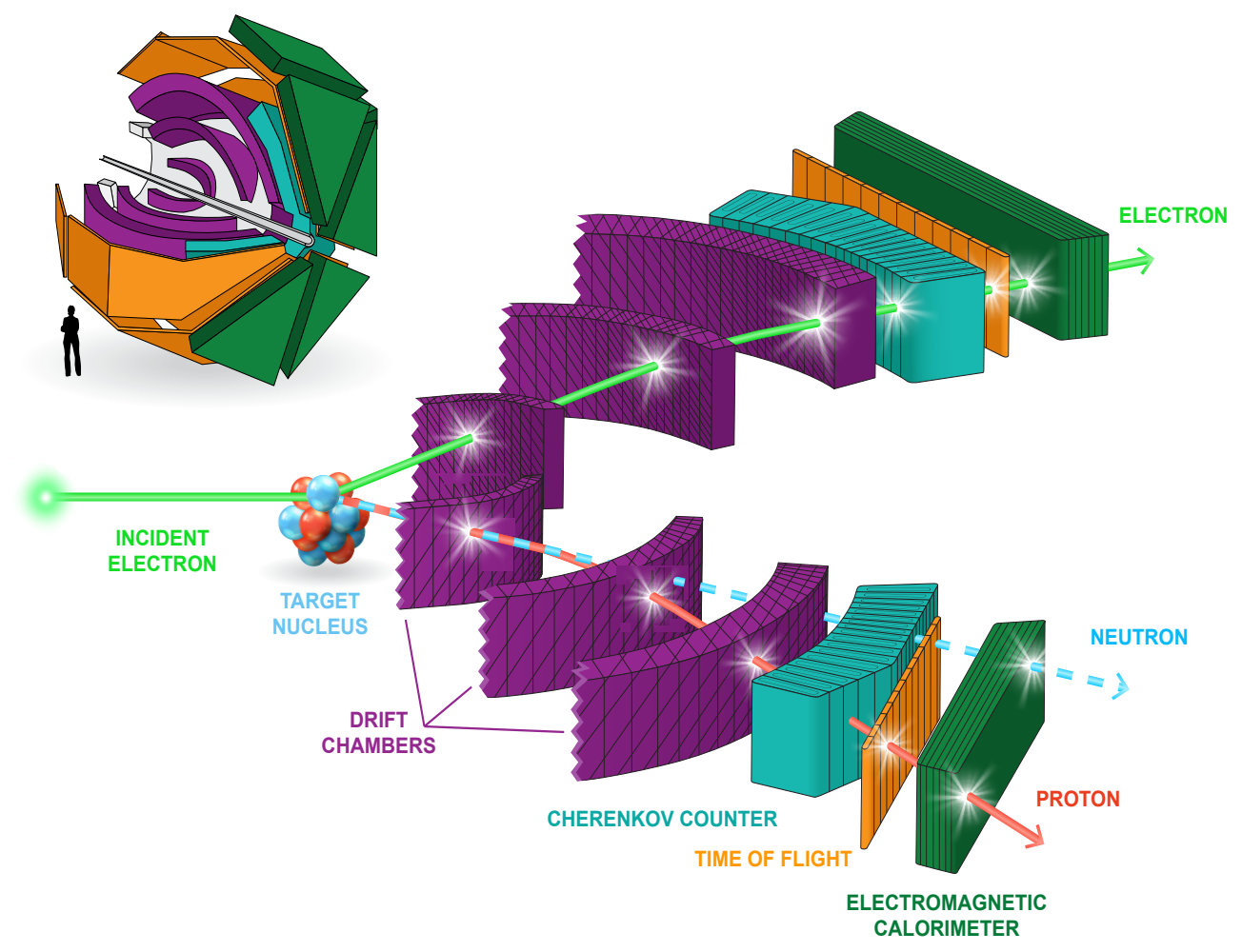

Figure 12: Center - Two segments of the CLAS spectrometer. Also shown are electrons traveling with energies of up to $6 \mathrm{GeV}$ hitting a nucleus, and knocking out individual protons and neutrons. Top left - Overview of the CLAS detctor with the electron beam travels along the grey pipe, hitting a target near the center of the spectrometer. Figure was adapted from 26 .

\subsubsection{Torus Magnet}

The torus magnet consist of six superconducting coils positioned in a toroidal geometry (see Fig. 13). The coils are placed perpendicular to the incident electron beam and they divide CLAS into six sectors, as described above. The coils which are made of $\mathrm{NbTi}$, are being kept in a stable temperature of $4.5^{\circ} \mathrm{K}$, using circulated cooling liquid. The magnet can produce a magnetic field changes from 2 Tesla in the forward direction, to 0.5 Tesla for backward angles (see Fig. 14). The design of the coils produces a magnetic field in the azimuthal direction, which bends the charged particles only in the polar angle, leaving the azimuthal angle almost unchanged. The magnetic field, generated by the torus is used to deflect charged particles and measure their momentum. Depending on the field direction, negatively charged particles are either in-bending or out-bending compared to the beam direction. The standard configuration for CLAS is the former. The relation between the magnetic field, $B$, the momentum, $P$, curvature $R$, and charge, $q$, of the particle is given by: $B \cdot R=\frac{P}{q}$. The radius of the curvature is measured by fitting the trajectory of the particle. 


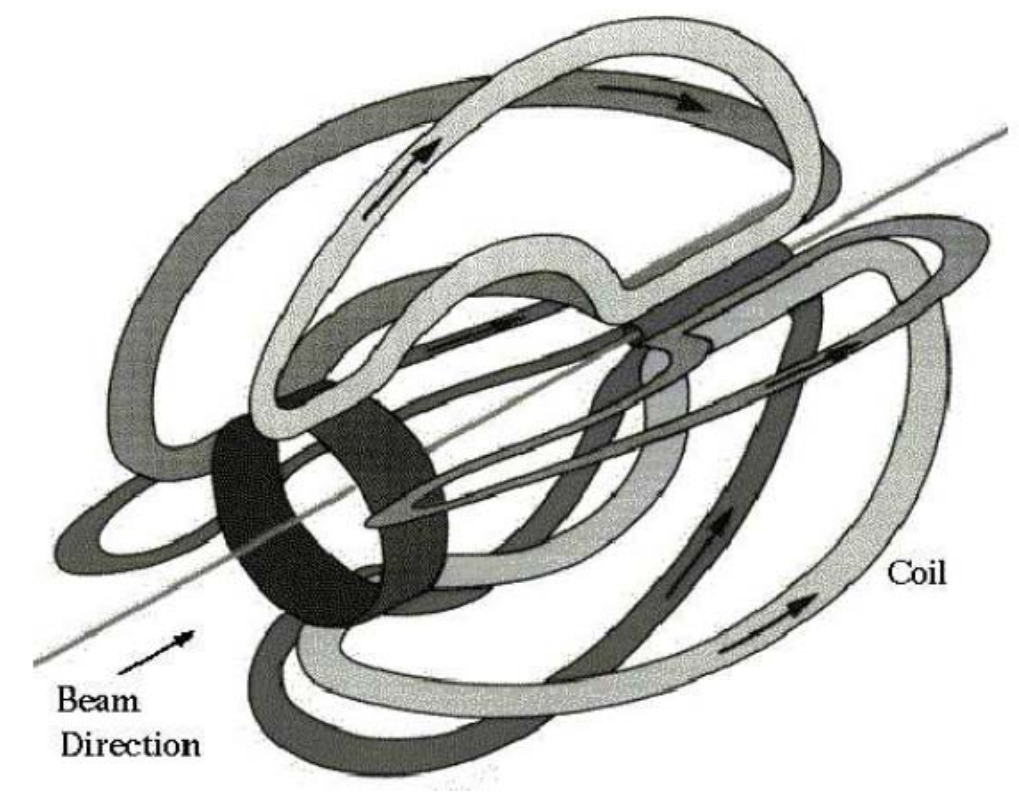

Figure 13: Schematic diagram of CLAS six superconducting coils that create the toroidal magnet. This figure was adapted from [40].

\subsubsection{Drift Chambers}

The purpose of CLAS Drift Chambers (DC) is to determine the trajectory in order to deduce the momentum of the charged particles. They are placed inside toroidal magnet which bends charged particles toward or away from the beamline depending on the charge of the particle. Their polar angular coverage is from $8^{\circ}$ to $140^{\circ}$, and the azimuthal coverage is $80 \%$. The missing polar and azimuthal coverages are due to the beam pipeline and the toroidal coils, respectively. The DC can track particles with a precision of $2 \mathrm{mrad}$, and determine the momentum of $1 \mathrm{GeV} / \mathrm{c}$ charged particles, with a resolution better than $1 \%$.

As mentioned before, the torus magnet divides CLAS into six azimuthal sectors. In each sector, the DC are divided radially into three regions (R1, R2, and R3), as can be seen in Figs. 12 and 14 . The three regions share the same material and wedge-like shapes, but they are subject to different strengths of magnetic field (see Fig. 14), and their size increases radially. R1 chambers are the closest to the target and are characterized by the smallest magnetic field. Moving further from the center of CLAS, are R2 chambers which, being at the middle of the coils, are characterized by the highest magnetic field. R3 chambers are located outside the torus magnetic field, in a relatively low magnetic field region. 


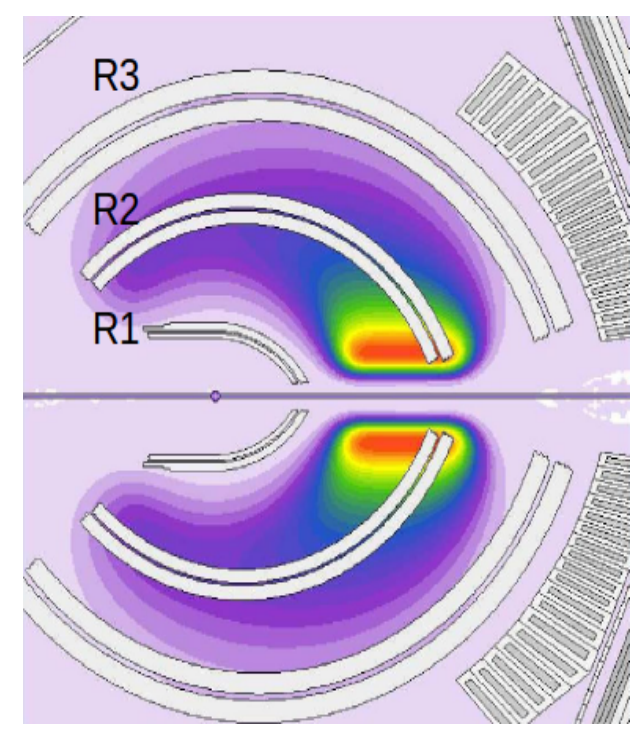

Figure 14: The three regions of the CLAS DC, and the torus magnetic field strength.

The DCs are constructed from two types of wires: sense and field wires. The sense wires are 20 $\mu \mathrm{m}$ diameter gold-plated tungsten. They are kept a positive potential, while the field wires are 140 $\mu \mathrm{m}$ diameter gold-plated aluminum, and are connected to a negative potential. These wires are arranged to form layers of hexagonal drift cells, as can be seen in Fig. 15. The layers are grouped to form two super-layers in each region (R1, R2, and R3) - one is co-axial with the magnetic field and the other is tilted at a $6^{\circ}$ stereo angle providing azimuthal information. The number and size of the cells in each region increase uniformly with the radial distance from the beamline. The volume of each DC is filled with a gas mixture of $90 \%$ argon, and $10 \% \mathrm{CO}_{2}$, which reduces multiple scattering, and provide high tracking efficiency $(>95 \%)$.

The tracking procedure within the DCs is performed in two steps: Hit-Based Tracking (HBT) and Time-Based Tracking (TBT). In the former, groups of continuous hits in each super-layer are recognized and a track segment from each group is constructed. Then, the segments from the individual super-layers are linked using a look-up table to obtain an initial estimate of the track by taking into account the magnetic field. During the TBT step, the Distances Of Closest Approach (DOCA) are calculated from the measured drift time of the hits, and compared to the estimated initial track. At last, a minimization DOCA procedure gives the final parametrization of the track, i.e., reconstruction of the momentum and the vertex. More details on the DC can be found in 9. 


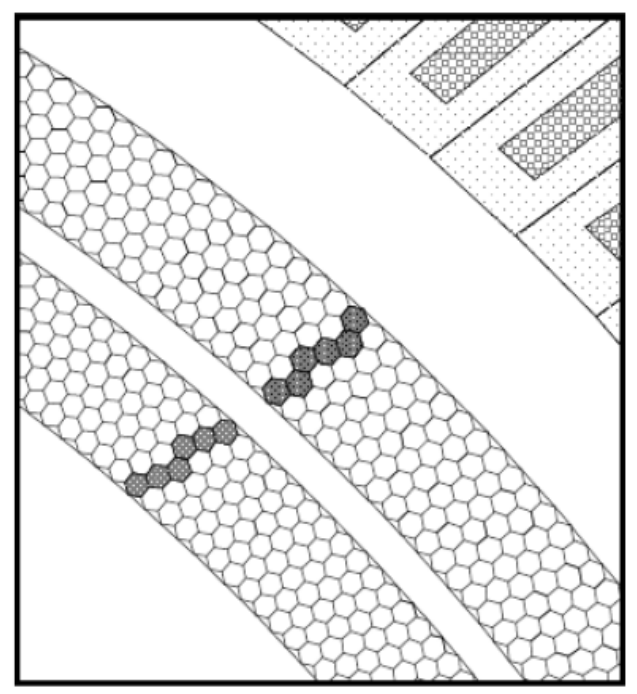

Figure 15: The hexagonal drift cells of the DCs. The sense wires are located in the center of each cell, while the field wires are located in the vertices of the hexagons. This figure was adapted from 9 .

\subsubsection{Cherenkov Counters}

The Cherenkov Counters (CCs) serve a dual function at CLAS: triggering on electrons and separating electrons from negative pions. They are placed in each one of CLAS sectors behind the third region of the DCs, as can be seen in Fig. 12. The CCs cover polar angles up to $45^{\circ}$, and $80 \%$ of the azimuthal angles. The CCs use the emission of Cherenkov light when a charged particle moves with a velocity greater than the speed of light in the medium. This is achieved when high-energy particles move in a medium having refraction index greater than one. The radiator gas used in CLAS CCs is perfluorobutance $\left(\mathrm{C}_{4} \mathrm{~F}_{10}\right)$, which has a refraction index of 1.00153. This gas provides a high photon yield and a threshold of $2.5 \mathrm{GeV} / \mathrm{c}$ to detect pions.

Figure 16 shows a schematic a view of a CCs for one sector of CLAS. Due to the size variation of each sector in the polar angle, each CCs system is divided into 18 regions, with two light collecting modules in each region. Figure 17 shows the inner structure of a CC module. It is made up of a mirror system which focuses the emitted light into Photomultiplier Tubes (PMTs). The PMTs are located in the fringe field region of the toroidal coils. They are surrounded with high permeability magnetic shields. Figure 17 also shows a schematic drawing of a typical crossing electron track. It emits photons which are focused by the mirrors before being collected by the PMT. In the fiducial region of the CCs, which are defined by the edges of the mirrors, the efficiency is very high (>98\%). More details on the CC can be found in [10]. 


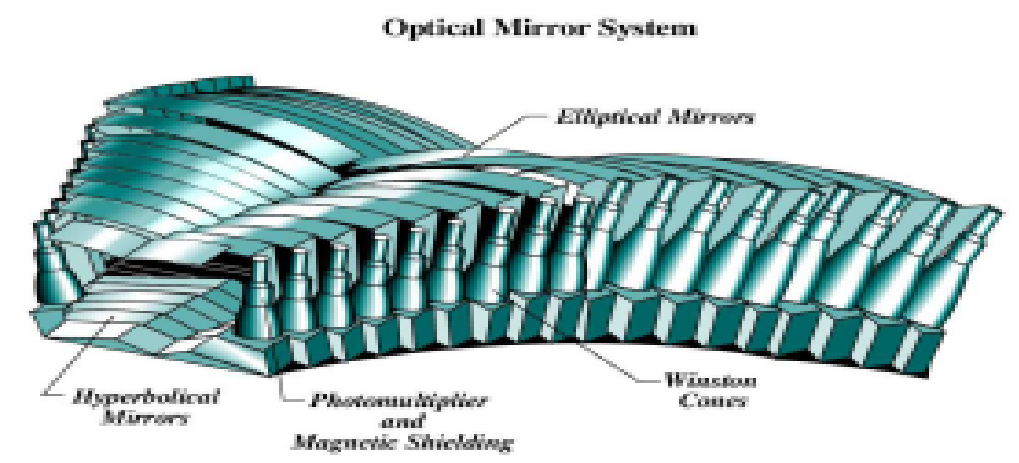

Figure 16: Schematic view of the CCs in one of the CLAS sectors. This figure was adapted from [10].

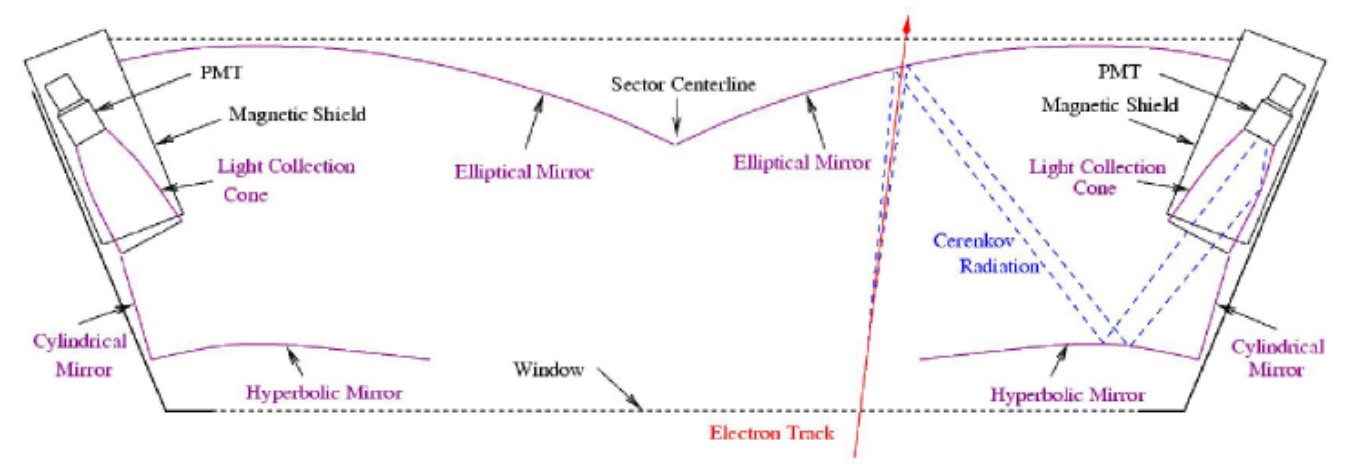

Figure 17: A cherenkov counter with a typical electron track crossing the radiator gas producing photo-electrons, detected by the PMTs. This figure was adapted from [5].

\subsubsection{The Time-Of-Flight counters}

The CLAS Time-Of-Flight (TOF) counters measure the time it takes a particle to reach it from the target. The time resolution is better than $300 \mathrm{ps}$. Using the TOF information and the length of the particle path, it is possible to calculate its velocity. By combining this information with the momentum of the particle, extracted from the track in the DCs, one can estimate the particle mass, and distinguish between different particles.

The location of the TOF is between the CCs and the calorimeters (see Fig. 12). There are six sectors, in each sector there are 57 scintillators bars (Bicron BC-408). Each scintillator bar has two PMTs at the ends. The time difference of the light arriving to these PMTs, allows the calculation of the position of the particle. All the scintillators have the same thickness of $5.08 \mathrm{~cm}$, but they have different lengths and widths depending on their positions. The length of the strips varies from $30 \mathrm{~cm}$ to $450 \mathrm{~cm}$. The width of the first 23 and the last four scintillators is $15 \mathrm{~cm}$, and the remaining scintillators are $22 \mathrm{~cm}$ wide. These scintillators are mounted in four panels. The first 23 scintillators are mounted in panel 1 and are referred to as forward-angle counters, which correspond to polar angles of less than $45^{\circ}$. The other scintillators are mounted in panels 2, 3, and 4 , and correspond to large-angle counters, covering polar angles between $45^{\circ}$ to $140^{\circ}$. Figure 18 shows schematically one sector of the TOF system. More details on the TOF can be found in 11. 


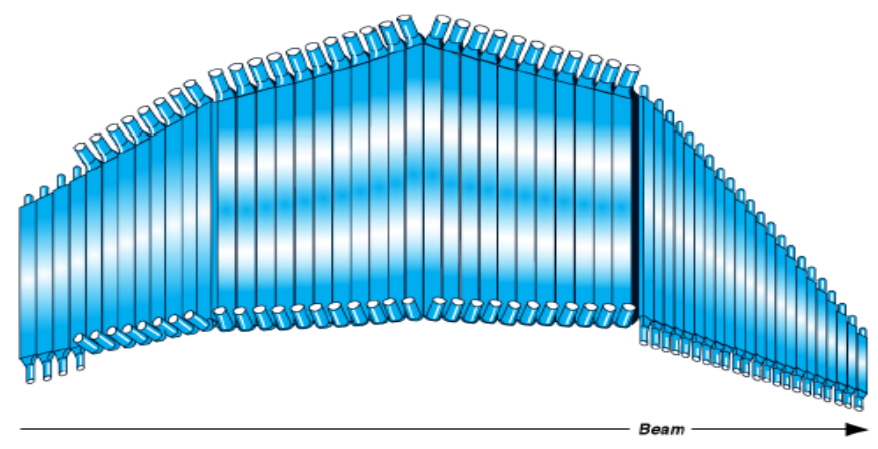

Figure 18: Schematic view of the TOF scintillators in one sector of CLAS. This figure was adapted from [11].

\subsubsection{Electromagnetic Calorimeter}

The forward Electromagnetic Calorimeter (EC) is the last layer of detectors in CLAS, located at a radius of about $5 \mathrm{~m}$ from the target, just after the TOF counters (see Fig. 12). Its primary purpose is to identify and trigger on electrons with energies greater than $0.5 \mathrm{GeV}$. It also allows CLAS to detect photons with energies greater than $0.2 \mathrm{GeV}$, reconstruct of $\pi^{\circ}$ and $\eta$ from their $2 \gamma$ decays, and detect neutrons. When charged or neutral particles pass through dense material, they will deposit their energy by producing electromagnetic showers or ionization. The amount of energy deposited as a function of the total momentum gives information about the particle identification. For example, the electron is a showering particle and the energy deposited in the EC is proportional to its total momentum, as opposed to a high momentum pion, which is a minimum-ionizing particle whose energy deposition is independent of its energy. This information provides capability for electron/pion separation. In addition, it is possible to identify neutrons in the EC by using the information from its time of flight.

The EC covers polar angles between $8^{\circ}$ to $45^{\circ}$, and has six identical sectors. The shape of each sector looks like an equilateral triangle to match the hexagonal geometry of CLAS. In each sector, there are 39 layers of alternating plastic scintillators and thick lead. Each scintillator layer consists of 36 scintillator bars, placed in parallel with respect to each other. These bars oriented with a $120^{\circ}$ relative angle in successive layers. The different scintillator orientations (views), labeled as U, V, and W, are shown in Fig. 19 (a). Each EC view is further divided into 13 layers to provide stereo information regarding the location of the energy deposition. These sub-layers are grouped into two independent regions (inner and outer) with a thickness ratio of 5:8 layers. This allows a longitudinal sampling of the shower to improve electron/hadron separation. This design allows a fine hit position reconstruction, as shown in Fig. 19 (b), and energy spread reconstruction. In this work we used the EC to detect neutron knocked from the nucleus in the $A\left(e, e^{\prime} n\right)$ reations, as discussed in details in Sec. 3.1.5. More details on the EC can be found in [12]. 


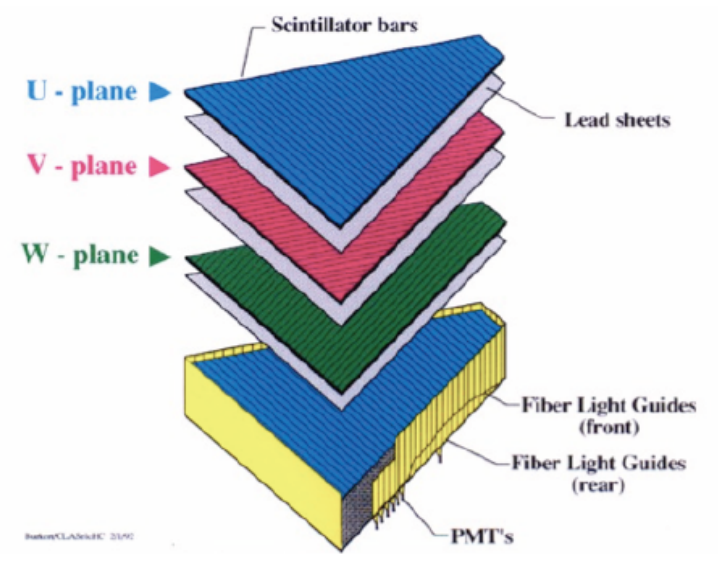

(a)

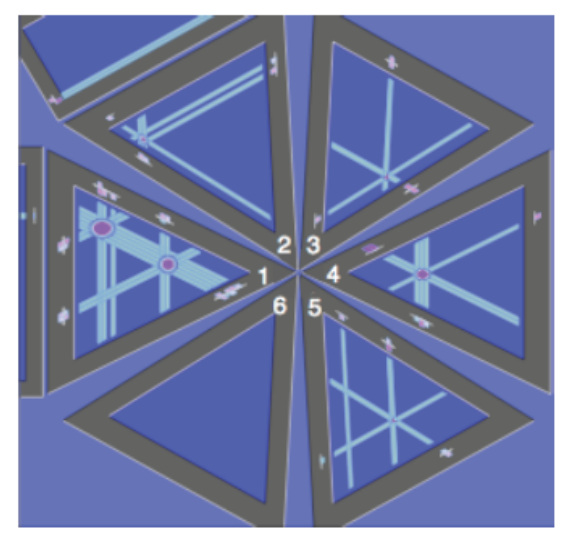

(b)

Figure 19: (a) U, V, and W orientations in an EC module. (b) hit reconstruction in the EC sectors. This figure was adapted from $[5$.

\subsection{The EG2 Experiment}

The data presented in this work were collected in 2004 in Hall-B of JLab, and were reanalysed as part of the data-mining initiative 21. CLAS measured data sets are stored by JLab, and the data-mining project gives access to it. It enables researchers to reanalyse existing data for any scientific purpose. Often, as in the case of the current work, the analyses that are being performed on these data sets are quite different from the original purposes of the experiments for which the data were collected. Below, we describe the experimental setup and the target assembly.

The experiment was performed using $5.014 \mathrm{GeV}$ electron beam (run-group EG2) with the CLAS spectrometer in its standard configuration, as described in Sec. 2.2. The EG2 run period used a specially designed double target, consisting of a liquid deuterium cryo-target, $\mathrm{LD}_{2}$, followed by a solid-target 22. The two targets were held simultaneously in the beam-line, and were separated by about $4 \mathrm{~cm}$. A remote-control computer based system allowed changing between six different solid targets (thin and thick $\mathrm{Al}, \mathrm{Sn}, \mathrm{C}, \mathrm{Fe}$, and $\mathrm{Pb}$ all with natural abundance) during the experiment (see Fig. 20). This double target setup was chosen originally to allow precise comparision of scattering off medium and heavy nuclei relative to deuterium. The original purpose of the experiment was to study hadronization (E02-104 exeperiment) and color transparency (E02110 experiment). The target thickness and the densities for the liquid and the solid targets were chosen to ensure equal luminosity, except for lead which was designed to produce half of the standard luminosity (see Table 3). The main data collected during the experiment was for a target configuration of $\mathrm{LD}_{2}+{ }^{12} \mathrm{C},{ }^{56} \mathrm{Fe}$, and ${ }^{208} \mathrm{~Pb}$. We analyzed electron scattering events from these three solid target and from the thick ${ }^{27} \mathrm{Al}$ target. Events from the deuterium target were used only for calibration of the neutron detection efficiency, and determination of the momentum resolution (see Sec. 3.1.5).

In the experiment the trigger to the Data-Acquisition (DAQ) was a detection of a scattered electron. The requirements were hits in the electromagnetic calorimeter and the Cherenkov counter detectors that produce signals above a certain thresholds. Once that condition was satisfied, data from all 
the different detectors of CLAS were recorded by the DAQ. This open trigger did not restrict the current work to specific kinematics or a presence of specific particles in the final state. A detailed description of the electron identification in CLAS can be found in Sec. 3.1.1.

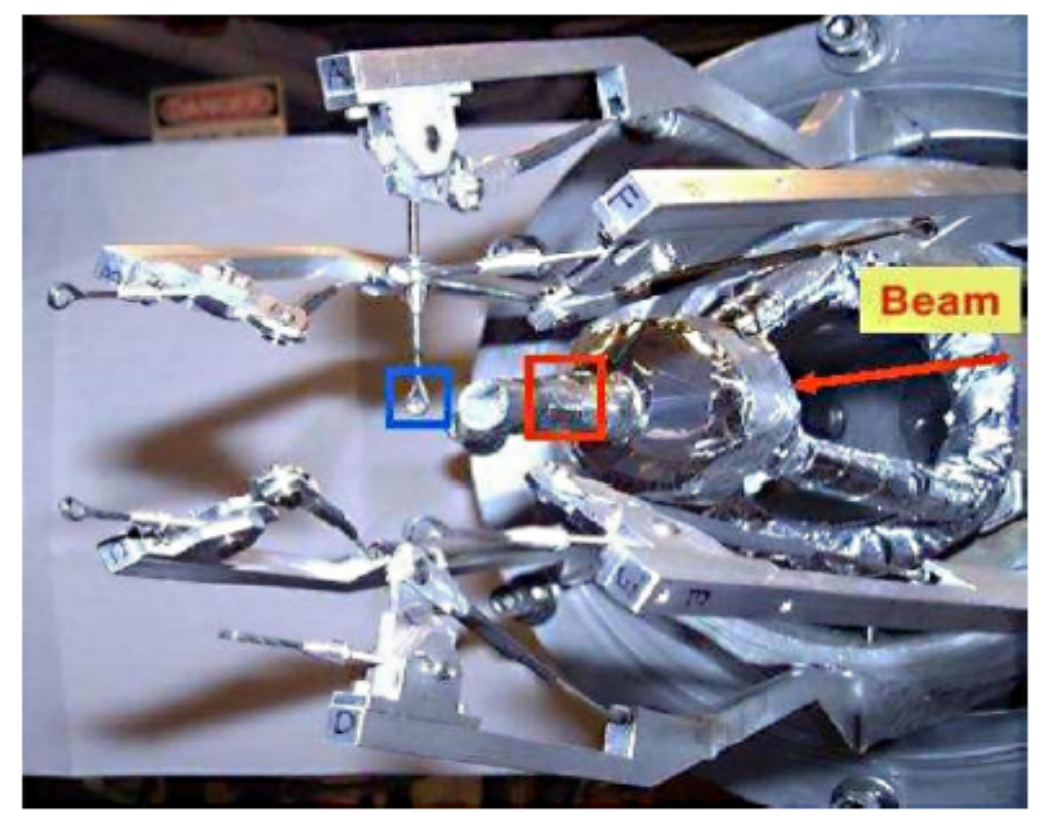

Figure 20: The EG2 target [22]. The red square shows the $\mathrm{LD}_{2}$ target cell. The blue square shows the solid target. The red arrow marks the beam direction. As can be seen, the beam passes throughout both targets simultaneously. This figure was adapted from [22].

\begin{tabular}{|c|c|c|c|}
\hline Target & Radius $[\mathrm{cm}]$ & Thickness $\left[\mathrm{g} / \mathrm{cm}^{2}\right]$ & Radiation Length \\
\hline \hline${ }^{12} \mathrm{C}$ & 0.15 & 0.3 & 0.009 \\
\hline${ }^{27} \mathrm{Al}{ }^{*}$ & 0.15 & 0.156 & 0.007 \\
\hline${ }^{56} \mathrm{Fe}$ & 0.15 & 0.315 & 0.023 \\
\hline${ }^{208} \mathrm{~Pb}$ & 0.15 & 0.159 & 0.025 \\
\hline
\end{tabular}

Table 3: The physical characteristics of four EG2 solid targets. * Thick target (see text). 


\section{Data Analysis}

In this chapter, we describe the particle identification, the calibration of the CLAS neutrondetection efficiency and momentum reconstruction, and the events selection criteria. The charged Particle Identification (PID) algorithms used in this work follow previous CLAS publications [6 7,8], so only the main aspects of the approach are presented here. More details are given on the neutron detection.

\subsection{Particle Identification}

\subsubsection{Electron Identification}

The electron candidates consist of all negatively charged tracks (as determined by their measured curvature in the CLAS tracking system [9]), with associated hits in the Cherenkov Counter (CC) [10, Time-Of-Flight (TOF) scintillator counters [11, and Electromagnetic Calorimeters (EC) 12. This sample contains contributions primarily from electrons and negatively charged pions. The identification of the electrons is done by applying selection cuts on the measured CC and EC signals:

- Since the EC has a poor respond close to its edges, reconstructed hit position is requested to be at least $10-\mathrm{cm}$ within the EC edges. This ensures that the produced shower is fully contained within the EC (see Fig. 21).

- More than 2.5 photo-electrons produced in the CC, see Fig. 22.

- Energy deposited in the EC inner part $\left(E C_{i n}\right)$ is greater than $50 \mathrm{MeV}$, see Fig. 23 .

- Energy deposited in both EC inner and outer $\left(E C_{t o t}\right)$ parts is proportional to the measured particle momentum, see Fig. 24.

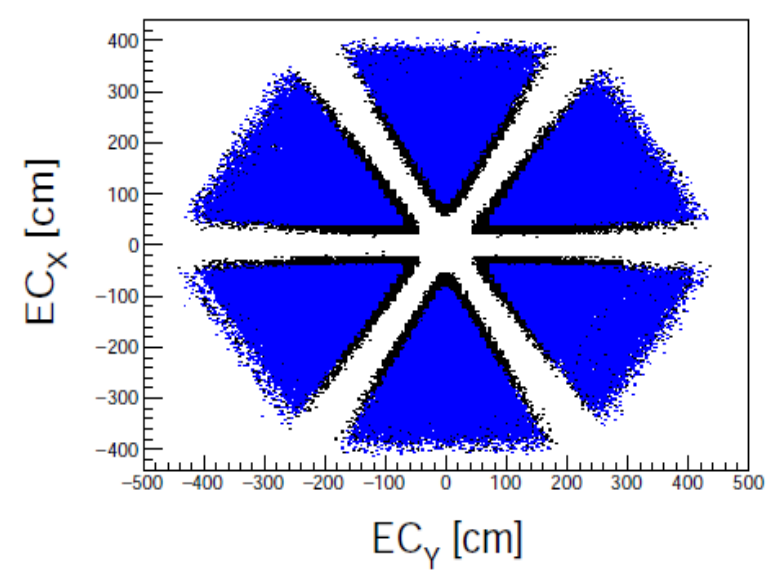

Figure 21: The EC fiducial region for electron candidates in the global $E C_{X}, E C_{Y}$ coordinates, where $E C_{Z}$ is the beam direction. Black: All events. Blue: After applying the fiducial cut (removing approximately $10 \mathrm{~cm}$ from the edges). 


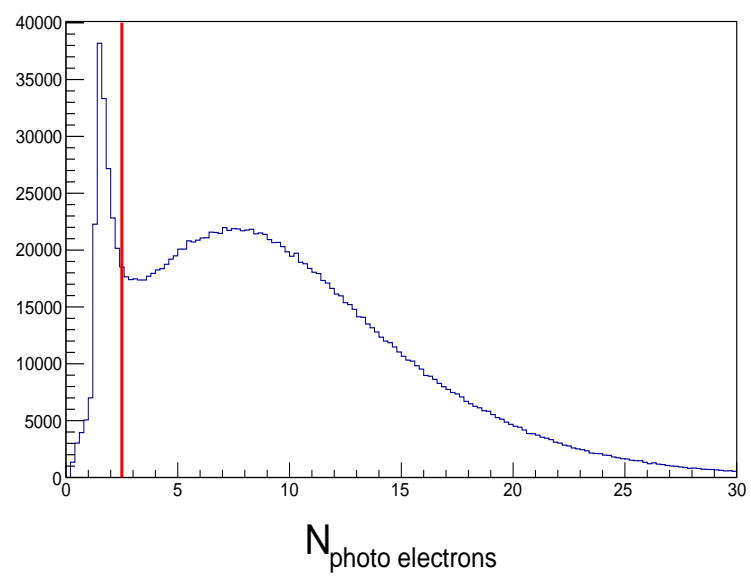

Figure 22: Distribution of the number of photo-electrons $\left(N_{\text {photo electrons }}\right)$ produced in CC by electron candidates which passed the EC fiducial cut. The applied $N_{\text {photo electrons }}>2.5$ cut is shown in red.

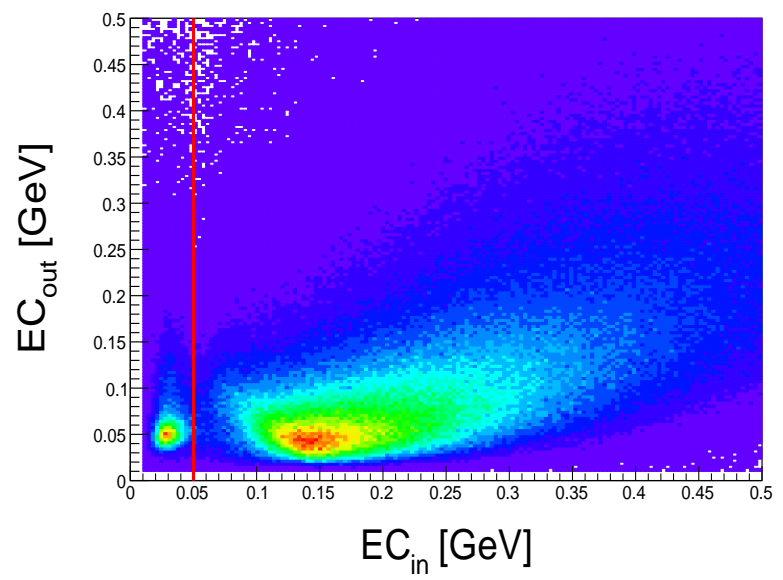

Figure 23: Energy deposited in the EC outer vs. inner parts. The shown events represent electron candidates that passed the EC fiducial cut, and the CC $N_{\text {photo electrons }}$ cut. The cut showed by the red line, $E C_{i n}>50 \mathrm{MeV}$, is used to separate electrons from pions. 

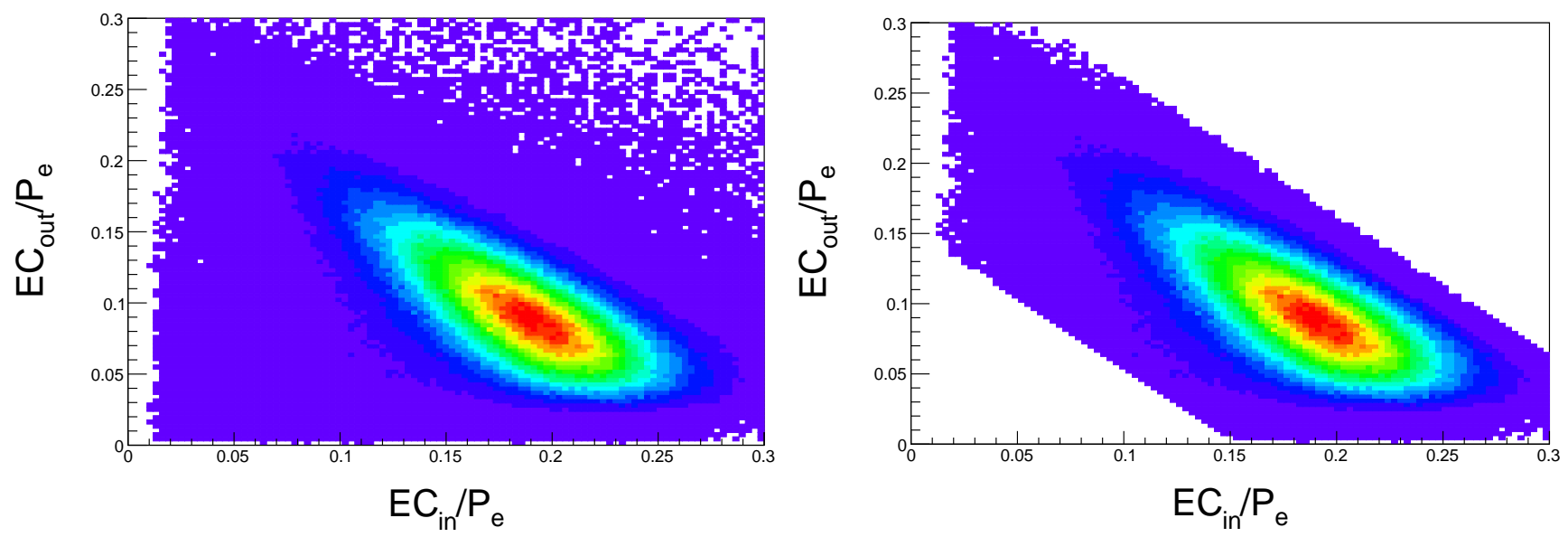

Figure 24: Momentum-normalized energy deposited in the EC outer vs. inner parts. Left: Events that passed

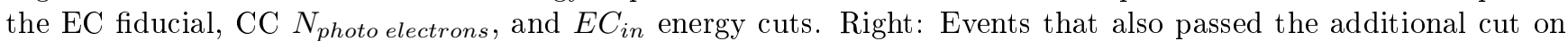
$\left(E C_{\text {in }}+E C_{\text {out }}\right) / p_{e}$.

\subsubsection{Proton Identification}

Protons are identified by comparing their measured arrival time at the TOF scintillator counters, to the expected time calculated for the momentum of positively charged particles as measured by the CLAS tracking system. The corrected proton vertex TOF is determined as the difference between the measured TOF of electrons and proton candidates and the calculated flight time using the reconstructed momenta and path lengths, assuming the positive particle has the mass of a proton. If the measured particle is a proton, this vertex TOF should be zero, within the experimental resolution. Figure 25 shows the corrected proton vertex TOF vs. the particle momentum for a sample of proton-candidates' tracks.

The corrected proton vertex TOF difference was binned into $50 \mathrm{MeV} / \mathrm{c}$ wide momentum bins and fitted with a Gaussian. For each bin the proton PID cut was chosen as being within 2 standard deviations $( \pm 2 \sigma)$ of the mean. A polynomial function was used to fit the $\pm 2 \sigma$ limits as a function of the particle momentum, up to $2.8 \mathrm{GeV} / \mathrm{c}$, to allow a continuous proton PID selection cut, see Fig. 25. Positively charged particles with corrected proton vertex TOF within the determined $\pm 2 \sigma$ limits are considered as protons for further analysis.

- Proton energy loss - The proton energy loss was studied in 14. For protons with momentum of $\sim 1 \mathrm{GeV} / \mathrm{c}$, it was shown to be about $2 \mathrm{MeV}$. For higher momentum protons, the energy loss increases, very slowly, and is still very close to $2 \mathrm{MeV}$. Therefore, for our analysis, this correction is negligible.

- Coulomb correction - We followed the procedure of [14. For scattering off a large- $Z$ nuclei, one needs to take into account the effect of coulomb distortions on the energies of charged particles as they transverse throughout the static electric field of the nucleus. A common method to correct the measured data for this effect is the Effective Momentum Approximation. In this approach the data is corrected event-by-event, for the coulomb repulsion of the knockout proton by the nucleus. This results in a shift in the energies measured by CLAS, that are summarized in Table 4. 


\begin{tabular}{|c|c|}
\hline Nucleus & $\Delta E[\mathrm{MeV}]$ \\
\hline \hline${ }^{12} \mathrm{C}$ & 2.9 \\
\hline${ }^{27} \mathrm{Al}$ & 5.6 \\
\hline${ }^{56} \mathrm{Fe}$ & 9.4 \\
\hline${ }^{208} \mathrm{~Pb}$ & 20.3 \\
\hline
\end{tabular}

Table 4: Values for the energy shifts due to coulomb distortions for each target nuclei.
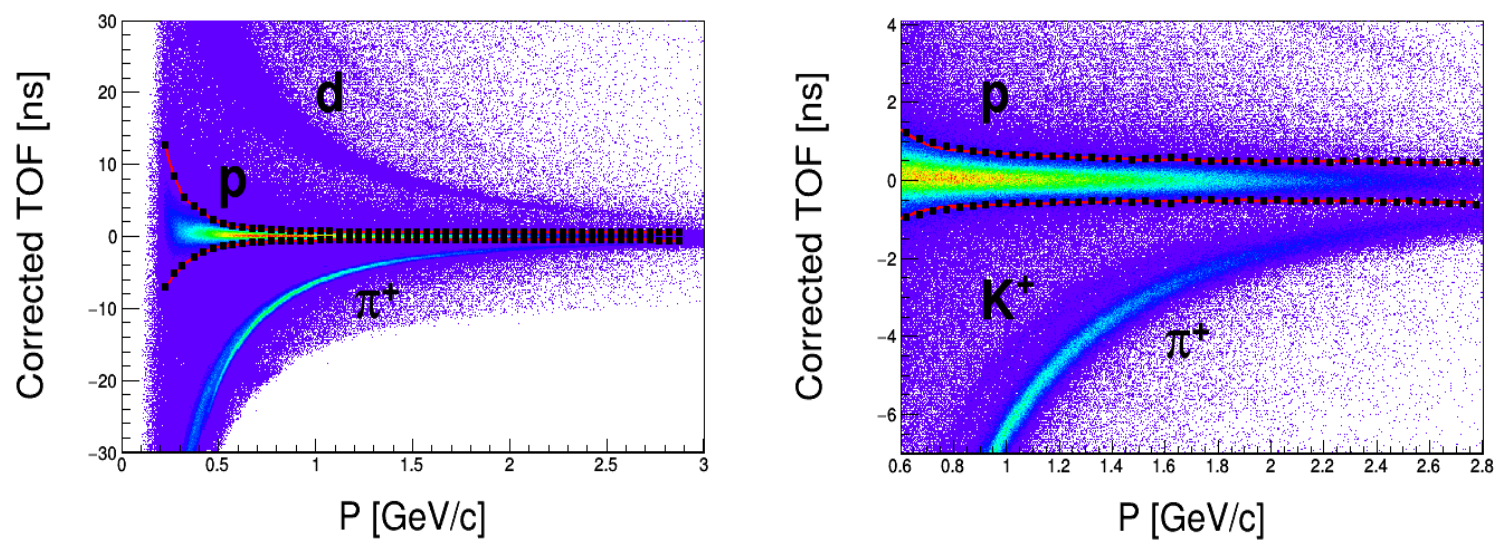

Figure 25: Corrected proton vertex TOF vs. momentum for a sample of proton-candidates' tracks. Left: the full coverage of the reconstructed proton momentum in CLAS. Right: High momentum region only up to $2.8 \mathrm{GeV} / \mathrm{c}$. The red lines show the polynomial parametrization to the black squares representing the $\pm 2 \sigma$ limits for each momentum bin used to identify protons. It should be noted that there is a constant background of random coincidence that uniformly populates the entire TOF spectrum. The use of color scale in the figure makes this background visible, even though it is less than one percent.

\subsubsection{Pions Identification}

Pions were only used to help calibrate the EC's neutron detection and momentum reconstruction efficiency using the kinematically complete $d\left(e, e^{\prime} p \pi^{+} \pi^{-} n\right)$ reaction. Pions were identified in a similar way as protons, using $\Delta \beta$, the difference between the measured and calculated particle velocity in speed of light units $(\beta=v / c)$, where the calculated velocity assumes the momentum as extracted from the curves in the magnetic field for particle with a pion mass (see Fig. 26). At large pion momenta there are some contributions from kaons. However, it is expected to be a small $3 \%$ contribution according to simulations and thus have a negligible impact on our results [13. 

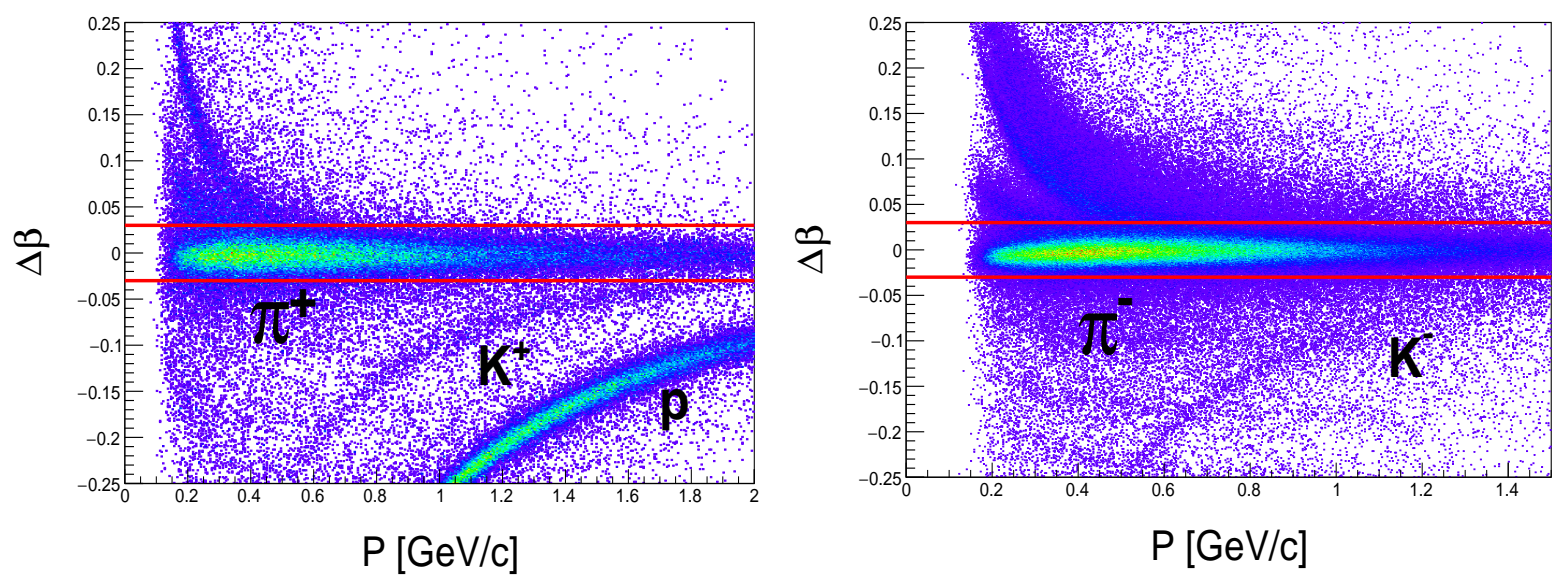

Figure 26: $\Delta \beta$ vs. momentum for a chosen sample of positively (left) and negatively (right) charged particles. The solid red lines represent the $|\Delta \beta|<0.03$ cut that is applied to select positive and negative pions.

\subsubsection{Vertex Reconstruction}

Electron scattering events originating from the EG2 dual targets were selected using vertex cuts adapted from Ref. 14. Figure 27 shows the reconstructed vertex distribution for the electrons and protons as detected in different CLAS sectors. The black lines represent the cuts used to select the liquid $(-28.5$ to $-32.5 \mathrm{~cm})$ and solid $(-22$ to $-26.5 \mathrm{~cm})$ targets. Events from the liquid deuterium target were used only for the calibration of the neutron detection efficiency. Figure 28 is the same as Fig. 27, but for negative and positive pions.
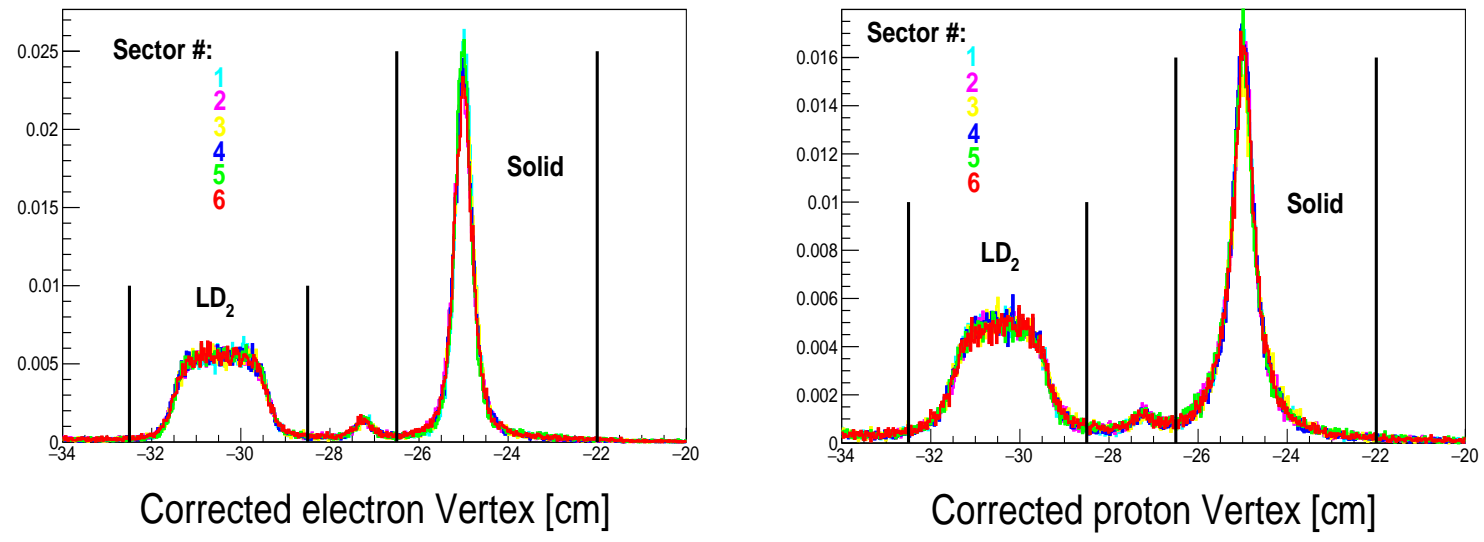

Figure 27: The corrected electron (left) and proton (right) target vertex distributions for each CLAS sector. All distributions are normalized to unity. The solid black lines represent the cuts defining the region of the solid and liquid targets. 

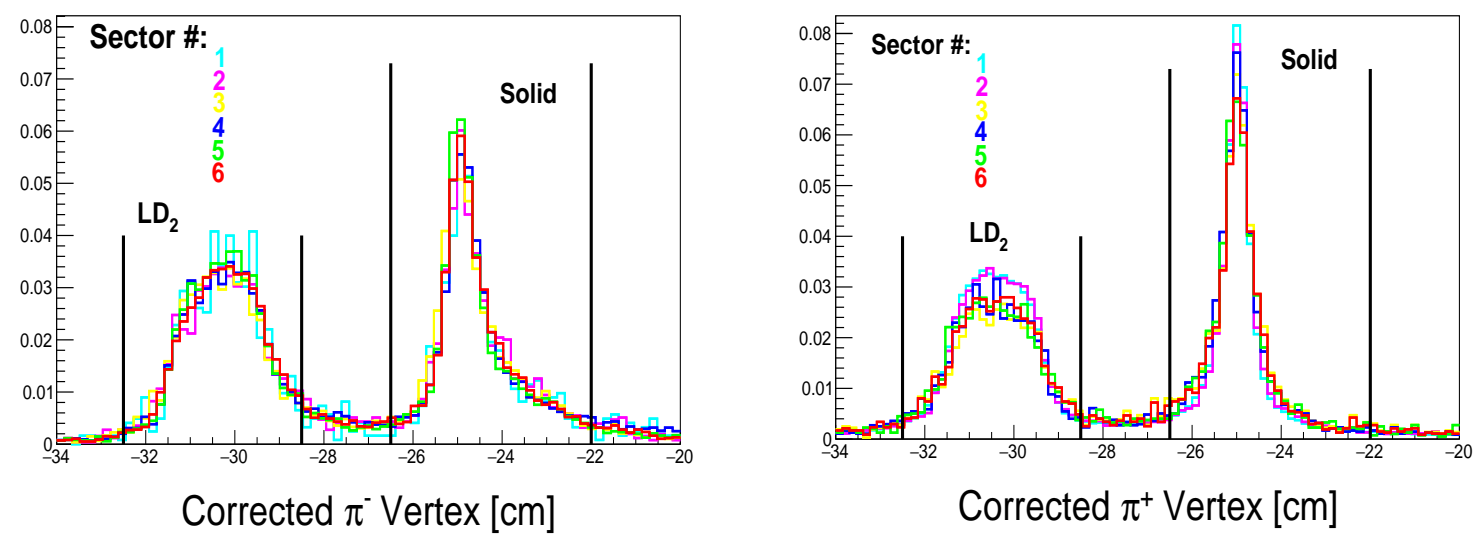

Figure 28: Same as Fig. 27 for pions.

\subsubsection{Neutron Identification, Detection Efficiency, and Momentum Reconstruction Resolution}

High momentum neutrons are detected in CLAS when they interact in the EC. We required neutrons to have a hit in the fiducial region of the EC with no associated charged track, no hit in the corresponding TOF detector, and with a velocity determined from its arrival time at the EC correspond to $\beta<0.936$. This cut accepts neutrons with momenta up to $2.34 \mathrm{GeV} / \mathrm{c}$ with minimal contamination from photons. The neutron momentum is calculated from the TOF of the neutral track.

The neutron-detection efficiency and momentum-reconstruction resolution are studied using exclusive $d\left(e, e^{\prime} p \pi^{+} \pi^{-} n\right)$ events where the neutron is detected in the EC. The neutron-detection efficiency is determined by comparing the number of such $d\left(e, e^{\prime} p \pi^{+} \pi^{-} n\right)$ events to the number of $d\left(e, e^{\prime} p \pi^{+} \pi^{-}\right) n$ events where the missing mass of the neutral particle, extracted using the measured charged particles, matches the neutron mass and the missing momentum vertex is pointing to the EC fiducial region. The neutron momentum-reconstruction resolution was determined by comparing the missing momentum of the $d\left(e, e^{\prime} p \pi^{+} \pi^{-}\right) n$ events with the momentum of the detected neutron. This process is described below.

Identification Of The Reaction $d\left(e, e^{\prime} p \pi^{+} \pi^{-}\right) n$

We identified $d\left(e, e^{\prime} p \pi^{+} \pi^{-}\right) n$ by considering all events in which an electron, a proton, a positive pion, and a negative pion were detected in coincidence by CLAS. We applied the following selection cuts to ensure the production of a neutron in the reaction:

- Interaction vertex cut - All particles are required to have a vertex within the region of the deuterium target, with a maximal vertex difference between any two particles less than 3 standard deviations (see Fig. 29).

- EC Fiducial cut - The direction of the missing momentum (see definition below) points more than $40 \mathrm{~cm}$ from the edges of the EC. 
- Missing mass cut - The missing mass of the reaction equals that of the neutron, within the measured resolution (see Fig. 30). A discussion about background subtraction in the missing mass distribution follows.

- Missing momentum cut - The magnitude of the missing momentum vector (see definition below) is greater than $500 \mathrm{MeV} / \mathrm{c}$ (see. Fig. 31).

The last three cuts are applied by calculating the missing four momentum of the reaction using its 3-momentum vector to define the "missing momentum" and its square to define the "missing mass". Here we define:

$$
p_{\mu}^{m i s s}=q_{\mu}+p_{\mu}^{d}-p_{\mu}^{p}-p_{\mu}^{\pi^{+}}-p_{\mu}^{\pi^{-}}
$$

where:

$q_{\mu}=(\omega, \vec{q})$ is the momentum transfer 4 -vector.

$p_{\mu}^{d}=\left(m_{d}, 0\right)$ is the 4-momentum of the deuterium target.

$p_{\mu}^{p, \pi^{ \pm}}$are the detected proton and pion 4-momenta.

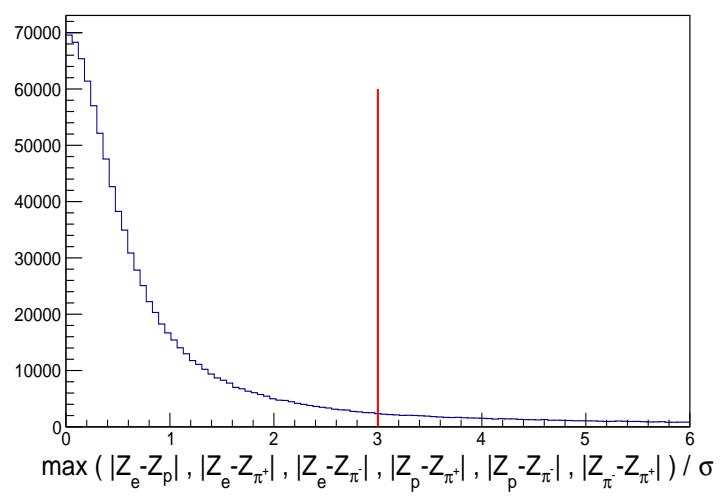

Figure 29: The maximum absolute difference between all charged particles vertex positions shown in units of $\sigma$, the combined vertex resolution for the four particles (electron, proton, and pions), by taking into account their measured angles and momenta. The red line shows the cut that is applied to reduce the background. Events shown are ones that passed the EC fiducial cut. 


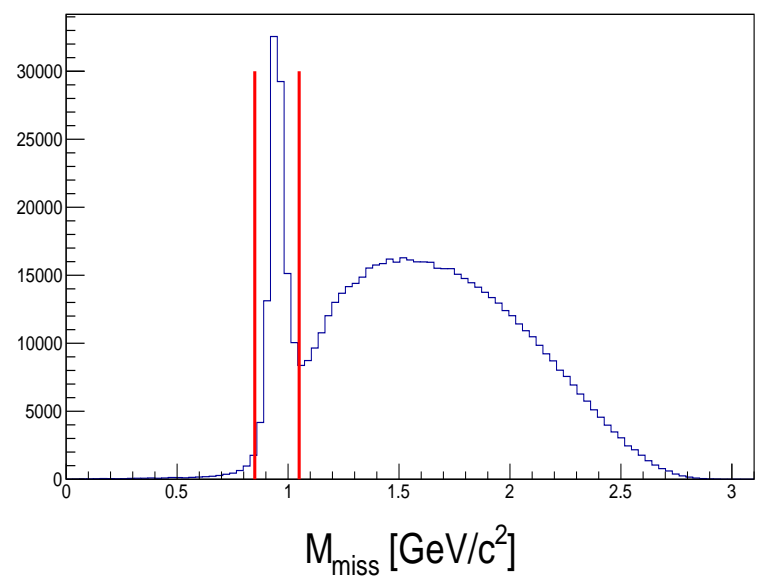

Figure 30: The $d\left(e, e^{\prime} p \pi^{+} \pi^{-}\right) X$ missing mass distribution. The red lines show the cut that is applied to select neutrons: $0.85<M_{\text {miss }}<1.05 \mathrm{GeV} / \mathrm{c}^{2}$. Events shown are ones that passed the EC fiducial cut and the interaction vertex cut.

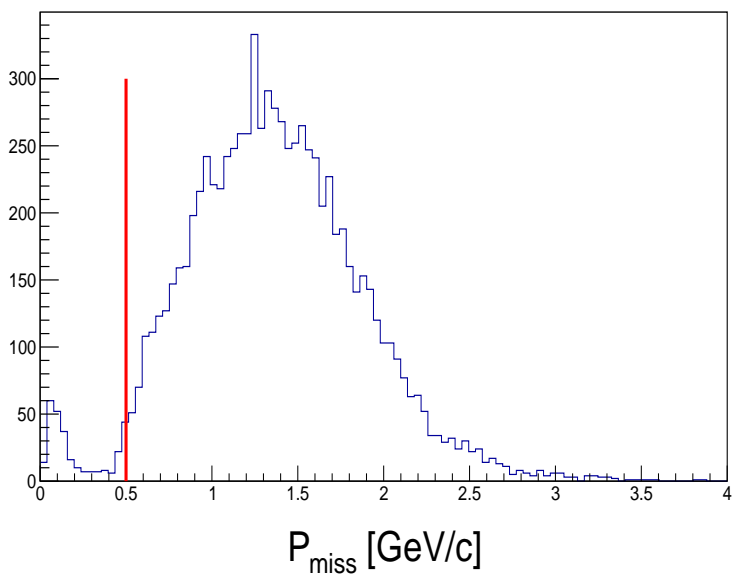

Figure 31: The missing momentum distribution. The red line shows the applied $P_{\text {miss }}>0.5 \mathrm{GeV} / \mathrm{c}$ cut.

Neutron Momentum Reconstruction, Calibration, and Resolution Extraction using the $d\left(e, e^{\prime} p \pi^{+} \pi^{-} n\right)$ Reaction

$d\left(e, e^{\prime} p \pi^{+} \pi^{-} n\right)$ events are a subset of the $d\left(e, e^{\prime} p \pi^{+} \pi^{-}\right) n$ events described above, in which a neutron was detected in the EC. To ensure that the detected neutron matches the inferred neutron, we compared the direction and reconstructed momentum of the detected neutron with that of the missing momentum vector. Specially, we considered only events in which the cosine of the relative angle between the measured neutron hit in the $\mathrm{EC}$ and the missing momentum vector is greater than 0.995 (see Fig. 32). Using these events, we compared the magnitude of the missing momentum with the reconstructed neutron momentum. The latter is determined by the neutral hit location in the EC and its measured interaction time. Fig. 33 (left) shows the correlation between the two momenta. Overall, the two are in good agreement besides the offset seen at high momenta. We corrected for this offset empirically by first determining a more suitable mean interaction location in the EC and then shifting the resulting reconstructed neutron momentum to match the missing momentum. Figure 33 (right) shows the correlation between the corrected and measured neutron 
momenta. The horizontal axis represents the neutron momentum as determined from the EC hit location and the TOF readings, and the vertical axis represents the calibrated momentum after the application of all corrections.

After calibrating the neutron momentum reconstruction we examined its resolution. This was done by fitting a Gaussian to the $\Delta p / p \equiv\left(p_{\text {measured }}-p_{\text {miss }}\right) / p_{\text {measured }}$ distribution for different neutron momentum bins. The obtained Gaussian width (standard deviation, $\sigma$ ) was taken as the momentum reconstruction resolution, which is shown in Fig. 34 as a function of the reconstructed neutron momentum in either the EC inner, outer, or both layers. This resolution extraction method leads to slightly worse resolution than the intrinsic neutron momentum resolution since it includes contributions from the missing momentum reconstruction resolution. This contribution was previously studied and found to be small [14].

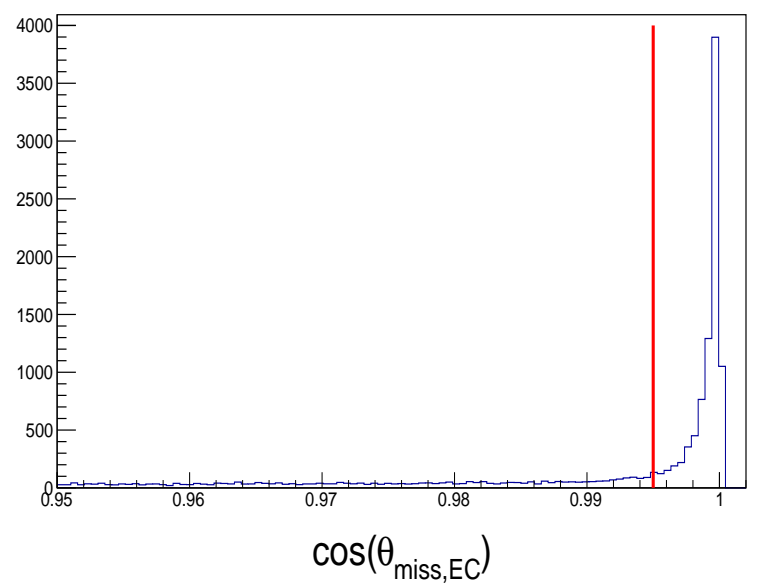

Figure 32: The cosine of the angle between the EC hit location and the missing momentum direction distribution for $d\left(e, e^{\prime} p \pi^{+} \pi^{-}\right) n$ events that have a neutral hit in the EC, passed the EC fiducial and $\beta$ cuts. The red line shows the $\cos \left(\theta_{\text {miss }, E C}\right)>0.995$ cut. 

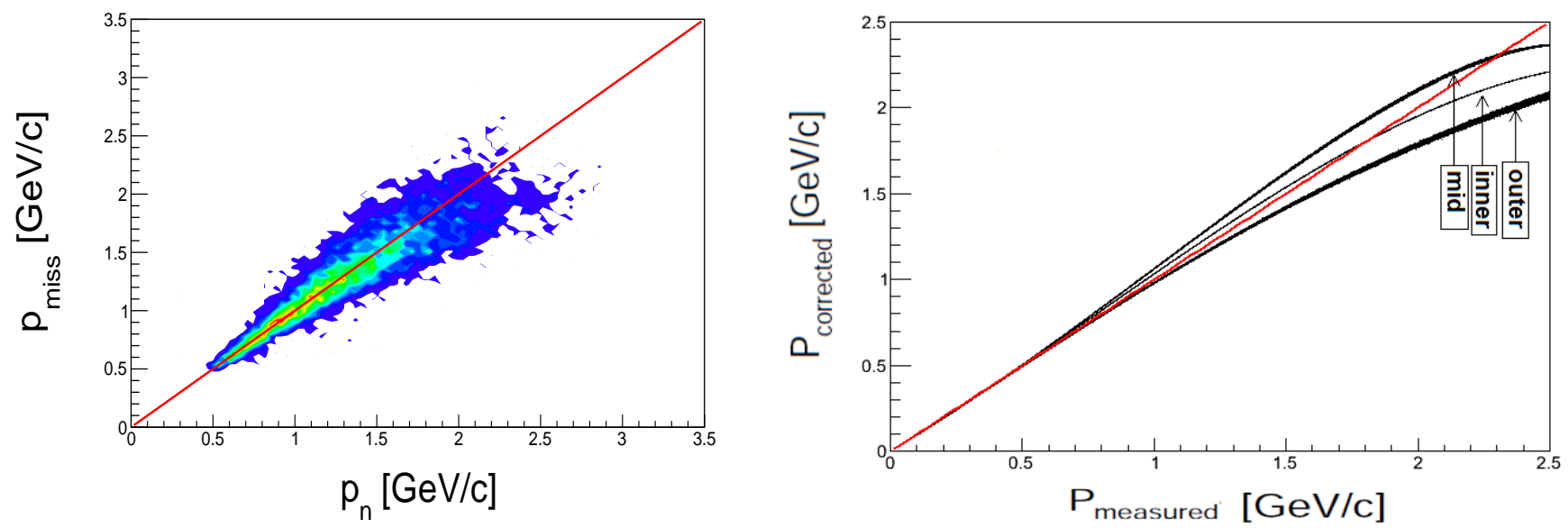

Figure 33: Left: The correlation between the missing momentum and the reconstructed neutron momenta before applying any corrections to the reconstructed momenta. Right: The correlation between the reconstructed and corrected neutron momentum for neutrons that deposit energy in either the inner, outer or both (mid) parts of the EC. The red line is meant to guide the eye and shows the case for a perfect calibration, i.e., measured $=$ corrected.

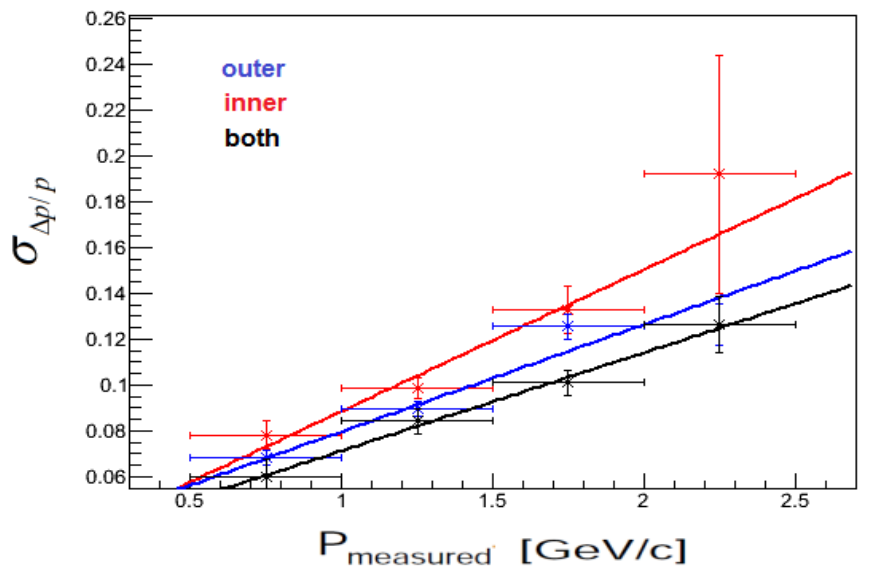

Figure 34: The EC momentum resolution (the width of the $\Delta p / p$ distribution) as a function of the measured neutron momentum.

\section{Neutron Detection Efficiency}

The neutron detection efficiency is defined as the ratio of $d\left(e, e^{\prime} p \pi^{+} \pi^{-} n\right)$ events in which a neutron was detected in the EC, relative to all $d\left(e, e^{\prime} p \pi^{+} \pi^{-}\right) n$ events:

$$
\epsilon=\frac{\# d\left(e, e^{\prime} p \pi^{+} \pi^{-} n\right)}{\# d\left(e, e^{\prime} p \pi^{+} \pi^{-}\right) n}
$$

Figure 35 shows the neutron detection efficiency as a function of the momentum for each sector of CLAS [5]. 
We parametrize the efficiency as a function of momentum using the following function:

$$
\epsilon= \begin{cases}a \cdot p_{n}+b & p_{n}<p_{0} \\ a \cdot p_{0}+b & p_{n} \geq p_{0}\end{cases}
$$

where $a, b$, and $p_{0}$ are the parameters of the fit. The lines in Fig. 35 show the fits of these parametrizations to the data for each sector. Table 5 lists the resulting fit parameters, the extracted efficiency at a typical momentum of $2 \mathrm{GeV} / \mathrm{c}$ and the overall quality of the fits.
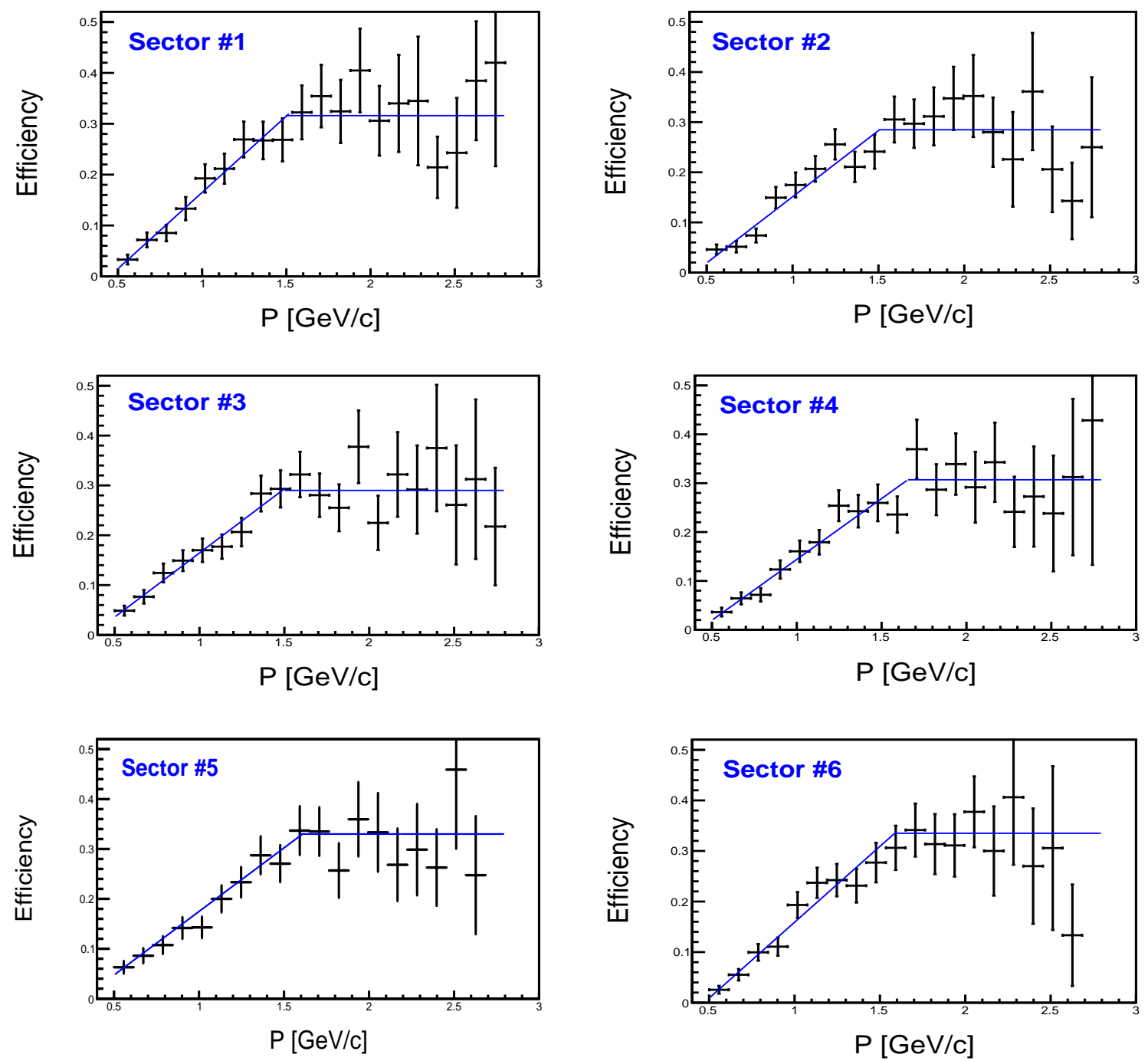

Figure 35: The neutron detection efficiency as a function of the momentum for each sector (points) together with a two-parameter fit to the efficiency for each sector (blue line). The fit parameters are listed in Table 5 . 


\begin{tabular}{|c|c|c|c|c|c|}
\hline Sector & $a[\mathrm{c} / \mathrm{GeV}]$ & $b$ & $p_{0}[\mathrm{GeV} / \mathrm{c}]$ & Efficiency at $2 \mathrm{GeV} / \mathrm{c}$ & $\chi^{2} / N D F$ \\
\hline \hline All & $0.28 \pm 0.01$ & $-0.11 \pm 0.02$ & $1.57 \pm 0.04$ & $0.33 \pm 0.01$ & 0.60 \\
\hline 1 & $0.30 \pm 0.03$ & $-0.13 \pm 0.03$ & $1.5 \pm 0.1$ & $0.32 \pm 0.03$ & 0.60 \\
\hline 2 & $0.26 \pm 0.02$ & $-0.11 \pm 0.03$ & $1.5 \pm 0.1$ & $0.28 \pm 0.02$ & 1.0 \\
\hline 3 & $0.26 \pm 0.03$ & $-0.09 \pm 0.03$ & $1.49 \pm 0.09$ & $0.29 \pm 0.02$ & 0.5 \\
\hline 4 & $0.24 \pm 0.03$ & $-0.11 \pm 0.02$ & $1.6 \pm 0.1$ & $0.31 \pm 0.03$ & 0.7 \\
\hline 5 & $0.26 \pm 0.04$ & $-0.08 \pm 0.03$ & $1.601 \pm 0.001$ & $0.33 \pm 0.02$ & 0.52 \\
\hline 6 & $0.30 \pm 0.03$ & $-0.14 \pm 0.03$ & $1.6 \pm 0.1$ & $0.33 \pm 0.03$ & 0.61 \\
\hline
\end{tabular}

Table 5: The fit parameters for the momentum dependence of the neutron detection efficiency and the efficiency at $2 \mathrm{GeV} / \mathrm{c}$.

\section{Accounting for Background Events in extracting the Efficiency}

Figure 36 shows the missing mass distributions of the denominator and the numerator of Eq. 7 for different missing momentum bins. Both distributions contain background events extending to neutron missing mass greater than $1.05 \mathrm{GeV} / \mathrm{c}^{2}$. We estimate the background fraction in each missing mass peak by fitting the full distribution to a sum of a Gaussian (signal) and a third order polynomial (background). We define the signal-to-background ratio as the ratio of the integral under the Gaussian $(S)$ to the integral of the total fitted function $(S+B)$ between the limits defined by the missing mass cut $0.85<M_{\text {miss }}<1.05 \mathrm{GeV} / \mathrm{c}^{2}$. Table 6 summarizes the ratios for different missing momentum bins. We corrected the efficiencies extracted above with the ratios listed in the fourth column of Table 6 .
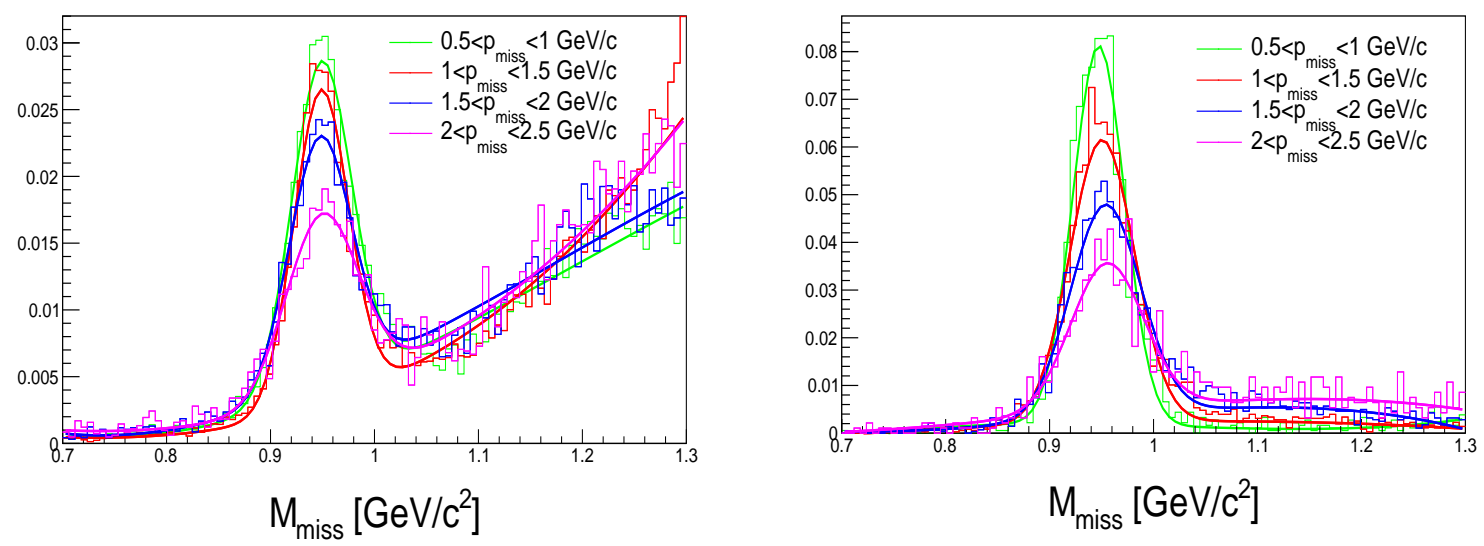

Figure 36: The missing mass distributions along with their fits for $d\left(e, e^{\prime} p \pi^{+} \pi^{-}\right) n$ events (left) and $d\left(e, e^{\prime} p \pi^{+} \pi^{-} n\right)$ events (right) for different missing momentum bins. 


\begin{tabular}{|c|c|c|c|}
\hline$\frac{S}{S+B}$ & $d\left(e, e^{\prime} p \pi^{+} \pi^{-}\right) n$ & $d\left(e, e^{\prime} p \pi^{+} \pi^{-} n\right)$ & $\epsilon_{\text {corrected }}$ \\
\hline \hline $0.5<p_{\text {miss }}<1 \mathrm{GeV} / \mathrm{c}$ & $69 \%$ & $86 \%$ & $1.24 \cdot \epsilon$ \\
\hline $1<p_{\text {miss }}<1.5 \mathrm{GeV} / \mathrm{c}$ & $66 \%$ & $80 \%$ & $1.21 \cdot \epsilon$ \\
\hline $1.5<p_{\text {miss }}<2 \mathrm{GeV} / \mathrm{c}$ & $62 \%$ & $77 \%$ & $1.22 \cdot \epsilon$ \\
\hline $2<p_{\text {miss }}<2.5 \mathrm{GeV} / \mathrm{c}$ & $61 \%$ & $70 \%$ & $1.15 \cdot \epsilon$ \\
\hline
\end{tabular}

Table 6: The signal-to-background ratios for different missing momentum bins, and their corrected efficiencies. See text for details.

\subsection{Identifying $A\left(e, e^{\prime} n\right)$ and $A\left(e, e^{\prime} p\right)$ Quasi-Elastic Events}

The main challenge in identifying low- $p_{\text {miss }}$ and high- $p_{\text {miss }}\left(e, e^{\prime} n\right)$ events is the poor neutron momentum resolution. To minimize this effect we optimized the event selection cuts using a sample of "smeared protons". We created this sample by smearing the momentum of the proton in each $\left(e, e^{\prime} p\right)$ event by the corresponding neutron momentum resolution.

To do this, for each $\left(e, e^{\prime} p\right)$ measured event we raffled several "smeared momenta" from a Gaussian distribution with a mean equal to the un-smeared proton momentum and a width equal to the neutron momentum resolution (as determined using the analysis of section 3.1.5). As the neutron momentum resolution is slightly different for neutrons that interact in the different EC layers, we generated the smeared protons in fractions that correspond to the probability of the neutrons depositing energy in the different EC layers. Figure 37 shows the difference between the original (un-smeared) and smeared proton momenta. Since different momenta have different resolutions, the resulting distribution is not a simple Gaussian but a sum of Gaussians.

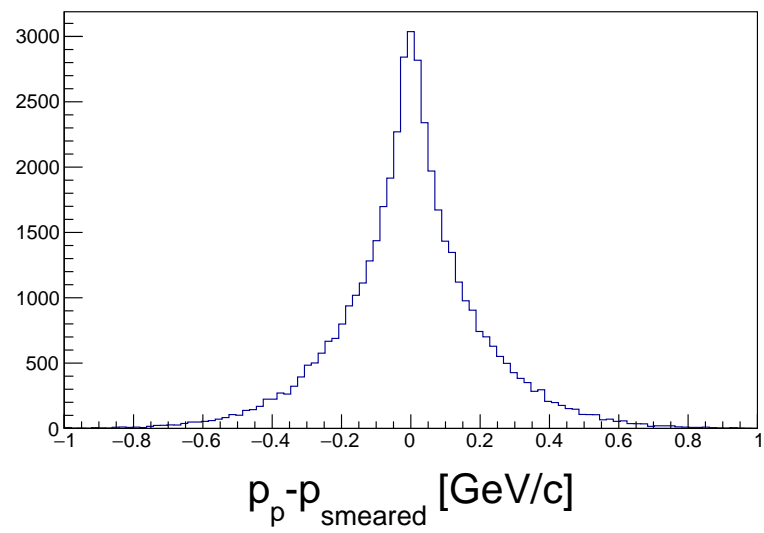

Figure 37: The difference between the original proton momentum $\left(p_{p}\right)$ and the smeared proton momentum $\left(p_{\text {smeared }}\right)$.

The strategy was to use these smeared-protons to help select $\left(e, e^{\prime} n\right)$ events. Therefore, we first matched the $\left(e, e^{\prime} p\right)$ and the $\left(e, e^{\prime} n\right)$ acceptances, by applying the following cuts:

- EC fiducial cut: As described in Sec. 3.1.1, the fiducial region in which the EC exhibits a valid response, is defined by removing $10-\mathrm{cm}$ from its edges. We applied this cut on the neutrons as well as the protons. In the latter case we did it by calculating the expected 
projection of the protons on the EC based on their measured scattering angle (before the protons curve in the magnetic field), and tracing the momentum vector from the origin to the EC face as a straight line.

- CLAS fiducial cut: The protons' fiducial cuts (see [15]) were applied also on the neutrons detected by the EC.

- Momentum cut: Same as for neutrons (see Sec. 3.1.5).

Through this chapter, unless stated otherwise, results are presented for ${ }^{12} \mathrm{C}$. Results for ${ }^{27} \mathrm{Al},{ }^{56} \mathrm{Fe}$, and ${ }^{208} \mathrm{~Pb}$ can be found in App. $\mathrm{A}-\mathrm{C}$ and in $\mathbf{1 5}$.

\subsubsection{Identifying High-Momentum (SRC) events}

High-momentum $\left(e, e^{\prime} N\right)(N=p / n)$ events are QE events with the electron being scattered off a nucleon with high-momentum $\left(k>k_{F}\right)$. Below we present the selection of $A\left(e, e^{\prime} n\right)$ events, using the smeared protons discussed above.

The identification of high-momentum $A\left(e, e^{\prime} p\right)$ events was previously studied in Ref. [6]. We therefore started by choosing an un-smeared set of $A\left(e, e^{\prime} p\right)$ events using the same selection criteria as Ref. [6], and optimized which cuts should be applied to the smeared protons in order to obtain a new set of events with as many $A\left(e, e^{\prime} p\right)$ events similar to Ref. [6] as possible (maximum efficiency) and as few other events as possible (maximal purity), while preserving the statistical precision of the data set. As our goal was to compare protons to neutrons, we always used the smeared proton sample in the analysis, whereas the un-smeared protons are used for validation and tests.

The event-selection cuts used in Ref. [6], and the modified cuts adapted for this work, are listed in Table 7. The main cut is on the missing momentum of the reaction. Assuming the PWIA for $\left(e, e^{\prime} N\right)$ scattering, the initial momentum of the reaction is equal to the missing momentum, defined as:

$$
\vec{p}_{\text {miss }}=\vec{p}_{N}-\vec{q}
$$

where $\vec{p}_{N}$ is the measured momentum of the scattered nucleon, and $\vec{q}$ is the momentum transfer vector. Additional cuts were applied to select high-momentum events, to suppress competing processes, such as Meson-Exchange Current (MEC), Isobar Configurations (IC), Single-Charge Exchange (SCX), and Final-State Interactions (FSI).

- $x_{B}$ cut:

To minimize FSI and IC, $x_{B}>1$ is required, i.e., the longitudinal initial momentum of the struck nucleon is directed opposite to the virtual photon. The MEC effect decreases as $1 / Q^{2}$ compared to SRC. Therefore requiring large $Q^{2}\left(>1 \mathrm{GeV}^{2} / \mathrm{c}^{2}\right)$ minimizes this effect. The chosen cut was $x_{B}>1.1$ (reduced from the 1.2 value used in Ref. [6] to increase statistics). Both cuts insure large $Q^{2}$, and therefore no additional cut was applied on it. Figure 38 shows the $Q^{2}$ distributions for (a) un-smeared protons, (b) the smeared protons (red) and neutrons (blue). 
(a)

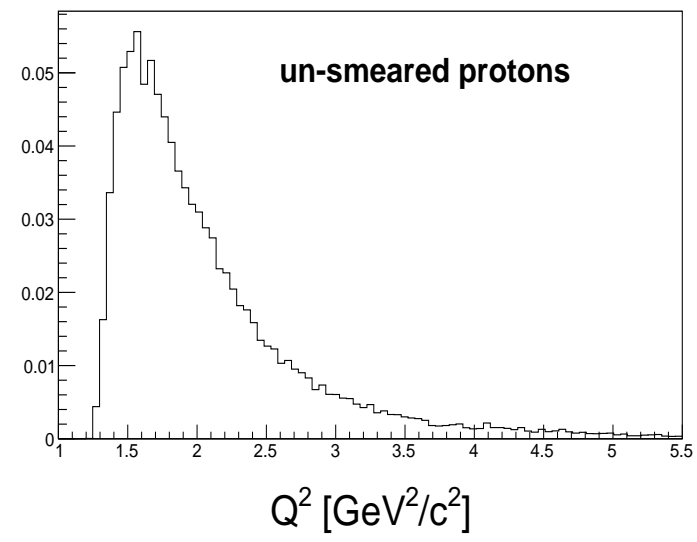

(b)

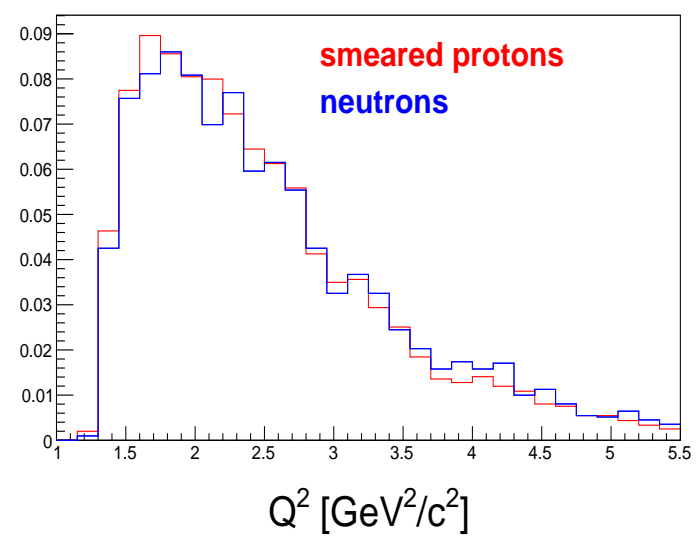

Figure 38: $Q^{2}$ normalized distributions for: (a) un-smeared protons events with $x_{B}>1.2$, and (b) smeared protons (red) and neutrons (blue) with $x_{B}>1.1$.

- Leading Nucleon Selection:

We wanted to identify the leading nucleon in the reaction, i.e., the nucleon that was struck by the virtual photon, rather than a recoil nucleon emerging due to other processes. With large $Q^{2}$, nucleons with high momentum and a small angle relative to the $\vec{q}$ are more likely to be the ones struck by the virtual photon. Figure 39 shows the relative angle between the detected nucleon and the $\vec{q}\left(\theta_{N q}\right)$ vs. the ratio between the detected nucleon momentum and the momentum transfer $\left(\left|\vec{p}_{N}\right| /|\vec{q}|\right)$, together with the chosen cuts $\left(\theta_{N q}<25^{\circ}, 0.62<\left|\vec{p}_{N}\right| /|\vec{q}|<0.96\right)$, for un-smeared protons [6]. Figure 40 is the same as Fig. 39 for smeared protons (a) and neutrons (b). We used the same angular cut, and loosened the momentum ratio cut to account for the momentum smearing $\left(0.62<\left|\vec{p}_{N}\right| /|\vec{q}|<1.1\right)$.

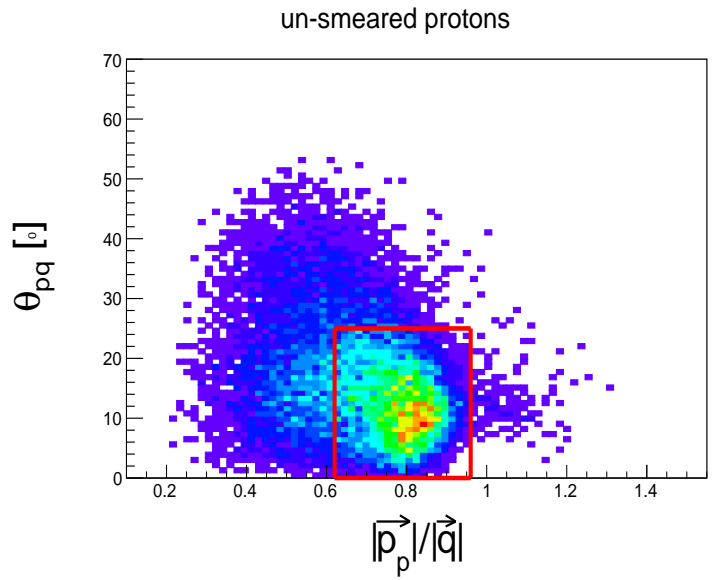

Figure 39: $\theta_{N q}$ vs. $\left|\vec{p}_{N}\right| /|\vec{q}|$ for un-smeared protons. The red box shows the cut applied to select leading protons in [6]. 
(a) smeared protons

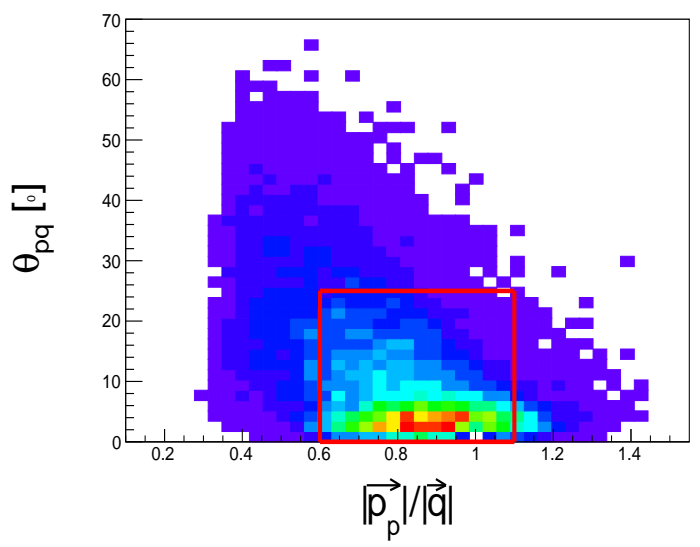

(b) neutrons

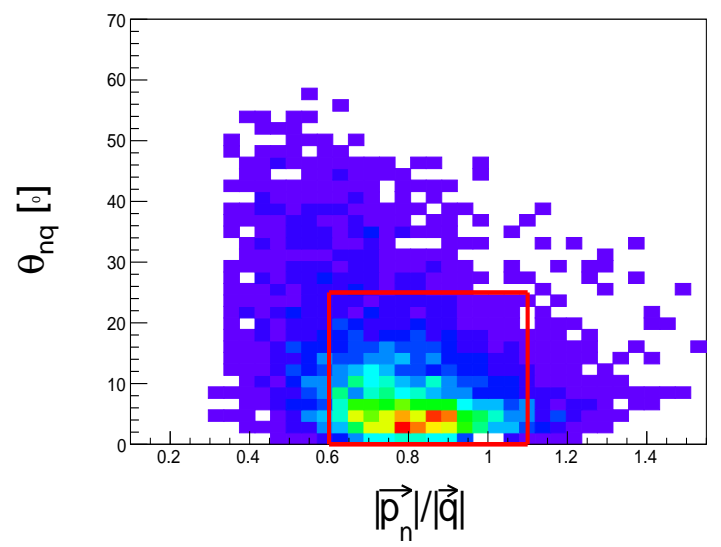

Figure 40: Same as Fig. 39 for smeared protons (a) and neutrons (b).

- Missing Momentum and Mass cuts:

The main cut selecting high-momentum events is on the missing momentum. Ref. [6] used a cut of $300<p_{\text {miss }}<1000 \mathrm{MeV} / \mathrm{c}$ for the un-smeared protons.

Assuming the electron scatters from a pair of nucleons at rest (i.e., $\vec{p}_{c . m .}=0$ ), then the missing mass of the $\left(e, e^{\prime} N\right)$ reaction is: $M_{m i s s}^{2}=\left(q+2 m_{N}-p_{N}\right)^{2}$, where $q$ is the 4-vector momentum transfer, $\left(2 m_{N}, \overrightarrow{0)}\right.$ is the 4 -vector of the pair, and $p_{N}$ is the 4 -vector of the struck nucleon. Due to the finite resolution of the detector and the c.m. motion of the pair, the distribution has a finite width. In order to avoid contamination from pion-production and delta-excitations, Ref. 6] used a cut of $M_{m i s s}<1100 \mathrm{MeV} / \mathrm{c}^{2}$.

The values of the missing momentum and mass cuts are very sensitive to the neutron momentum reconstruction resolution. We therefore optimized these cut values using the smeared-proton event sample by varying the cut values and examining:

- False positive probability - The fraction of events that do pass the missing momentum and mass cuts using the smeared proton momentum, but do not pass the cuts of Ref. 6] when using the un-smeared momentum. Reducing this fraction maximizes the "purity" of the sample.

- False negative probability - The fraction of events that do not pass the missing momentum and mass cuts when using the smeared proton momentum, but do pass the equivalent cuts of Ref. 6] when using their un-smeared momentum. Reducing this fraction maximizes the "efficiency" of the sample.

Figure 41 shows the false positive (left) and false negative (right) probabilities as a function of the lower missing-momentum cut for different missing-mass cuts applied using the smeared-proton momentum (the upper missing-momentum cut was chosen to be $p_{\text {miss }}<1000 \mathrm{MeV} / \mathrm{c}[15]$ ). The circle marks the chosen cut value for which both probabilities are comparable, low $(\sim 15 \%)$ and still have reasonable statistics $\left(p_{\text {miss }}>400 \mathrm{MeV} / \mathrm{c}, M_{\text {miss }}<1175 \mathrm{MeV} / \mathrm{c}^{2}\right)$. 
Figure 42 and 43 show the kinematical distributions of the resulting ${ }^{12} \mathrm{C}\left(e, e^{\prime} n\right)$ and ${ }^{12} \mathrm{C}\left(e, e^{\prime} p\right)$ events. Figures 44 and 45 show the same distributions for $\left(e, e^{\prime} n\right)$ scattering events for all targets.

\begin{tabular}{|c|c|}
\hline This analysis & Proton analysis [6] \\
\hline \hline$x_{B}>1.1$ & $x_{B}>1.2$ \\
\hline $0.62<\left|\vec{P}_{N}\right| /|\vec{q}|<1.1$ & $0.62<\left|\vec{P}_{p}\right| /|\vec{q}|<0.96$ \\
\hline$\theta_{N, q}<25^{\circ}$ & $\theta_{p q}<25^{\circ}$ \\
\hline$M_{\text {miss }}<1175 \mathrm{MeV} / \mathrm{c}^{2}$ & $M_{\text {miss }}<1100 \mathrm{MeV} / \mathrm{c}^{2}$ \\
\hline $400<p_{\text {miss }}<1000 \mathrm{MeV} / \mathrm{c}$ & $300<p_{\text {miss }}<1000 \mathrm{MeV} / \mathrm{c}$ \\
\hline
\end{tabular}

Table 7: The selected cuts of this analysis ( $1^{\text {st }}$ column), and the cuts used in the knockout proton analysis 6 ( $2^{\text {nd }}$ column).
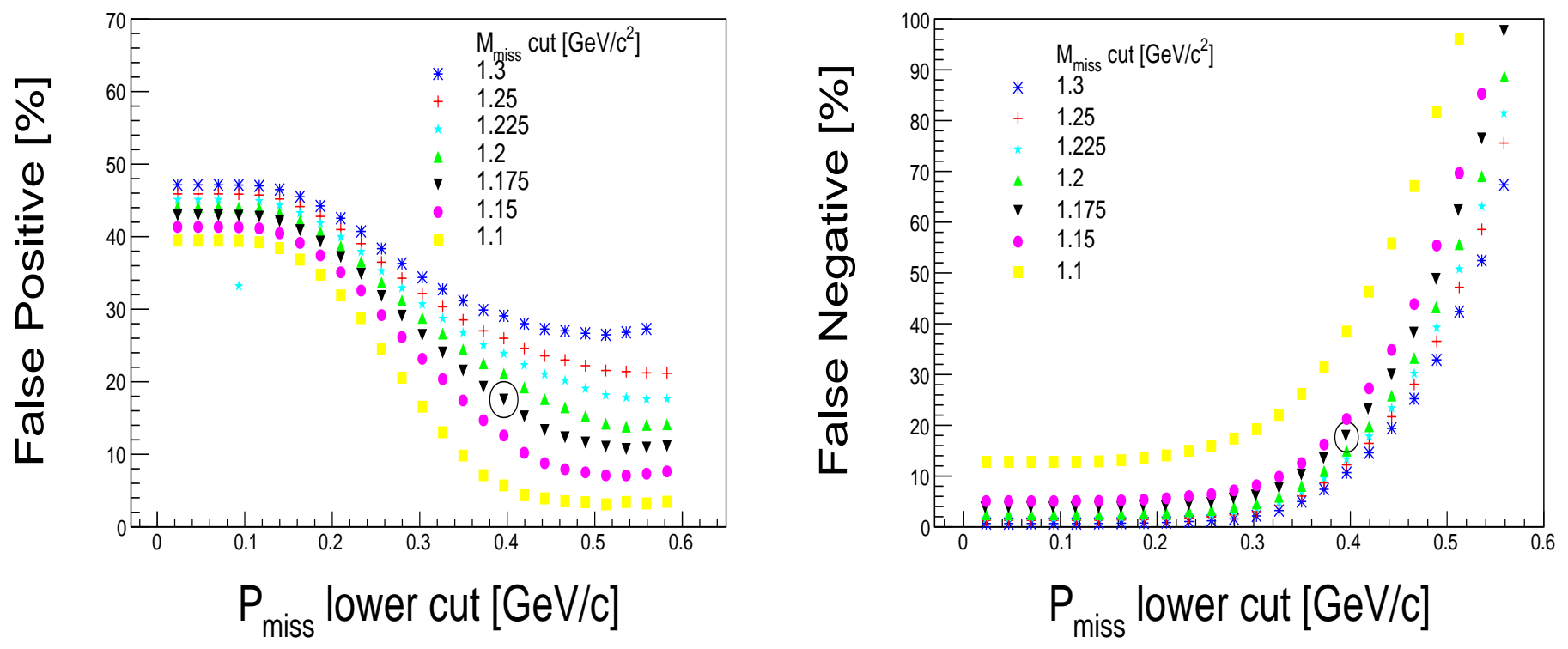

Figure 41: The false positive (left) and negative (right) probabilities, as a function of the smeared protons lower missing momentum cut for different cuts on the missing mass. The black circles represent the chosen cuts. See text for details. 
(a)

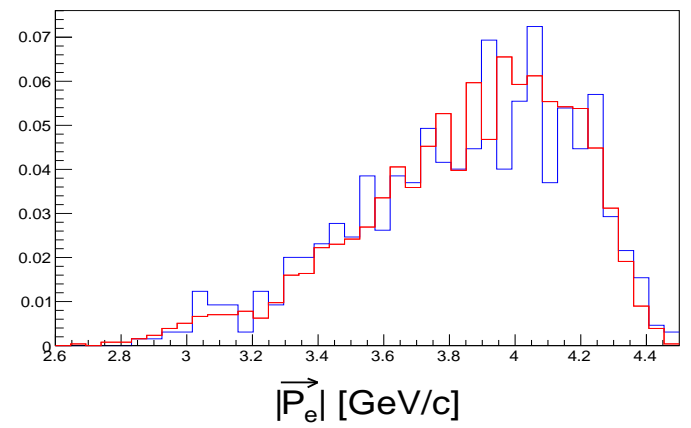

(c)

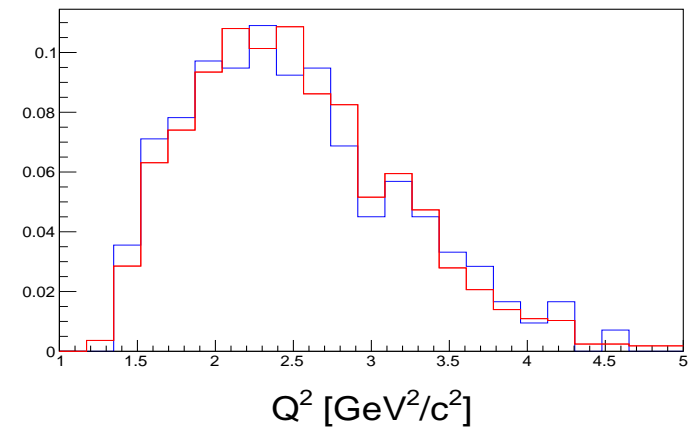

(b)

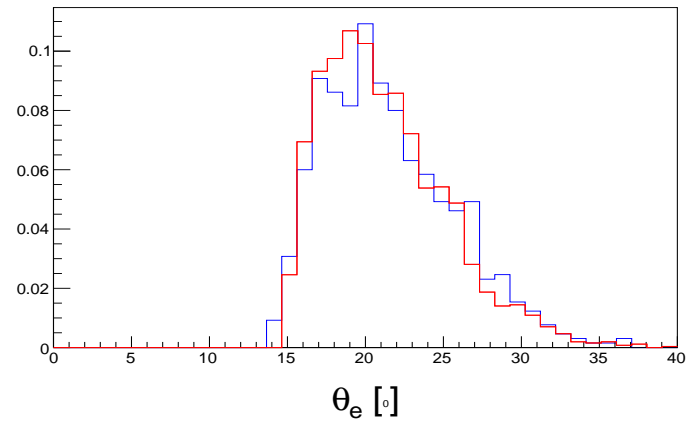

(d)

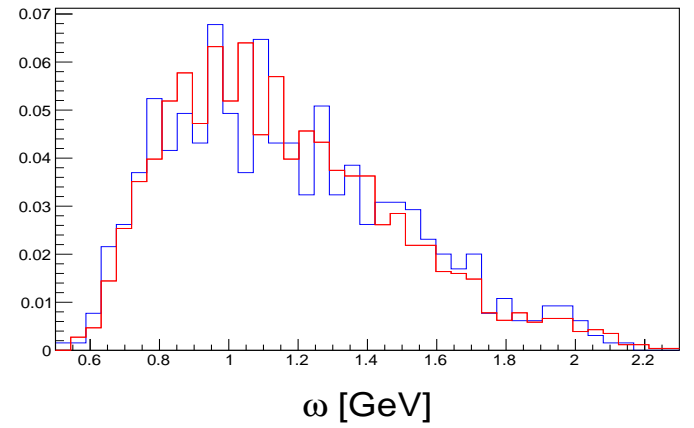

(e)

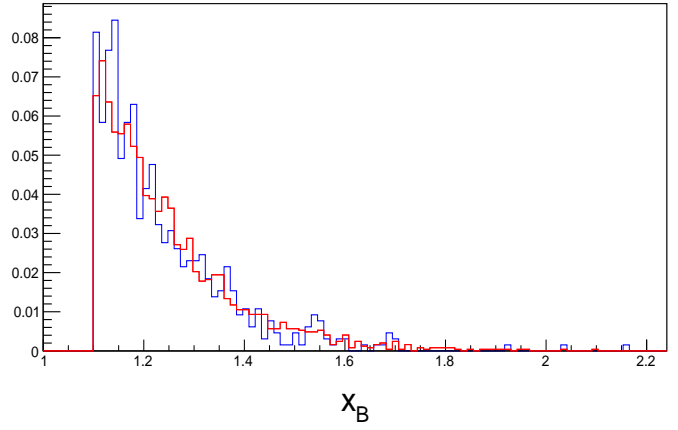

Figure 42: The electron kinematic variables for smeared protons (red) and neutrons (blue) after applying the high missing momentum cuts. Shown are (a) the electron momentum, (b) the electron scattering angle, (c) $Q^{2}$, (d) $\omega$, and (e) $x_{B}$. All distributions are normalized to unity such that only their shapes are to be compared. 
(a)

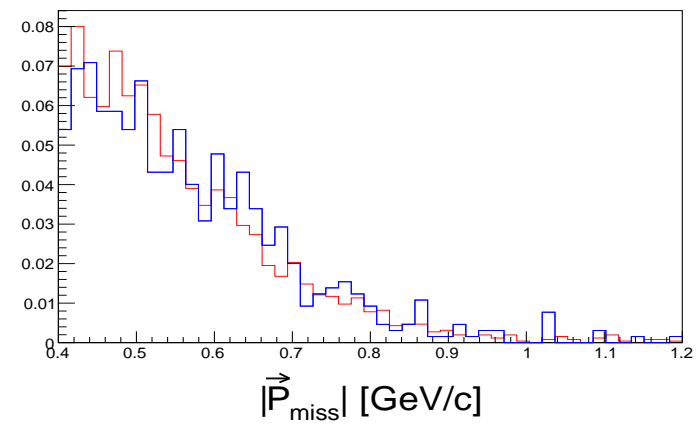

(c)

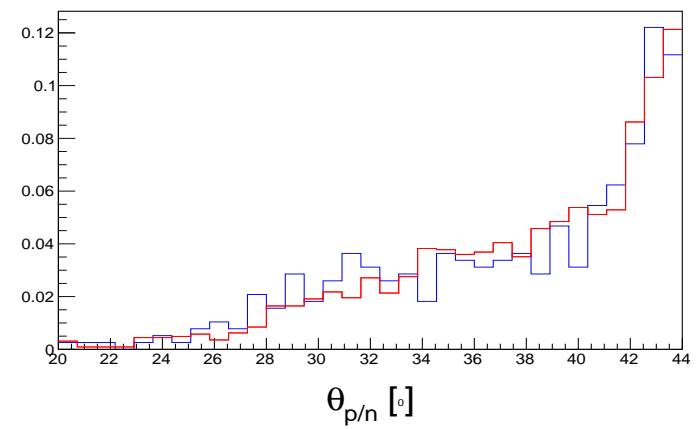

(b)

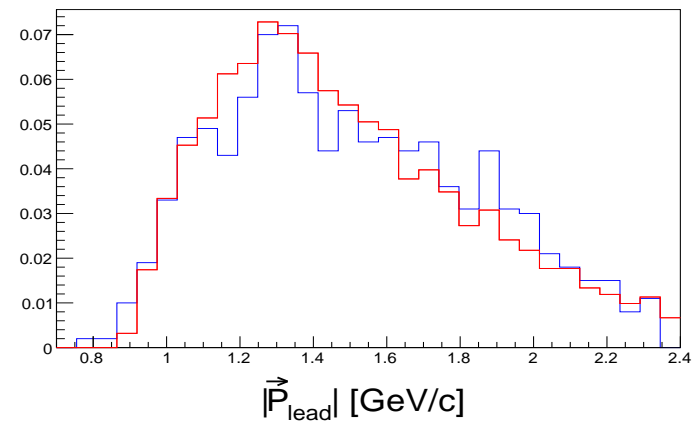

(d)

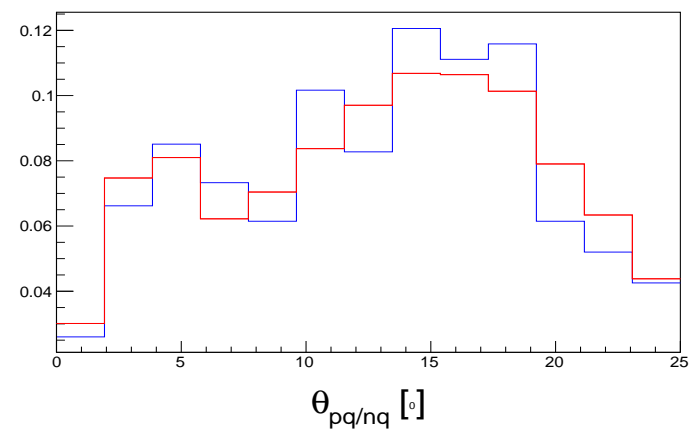

Figure 43: The smeared-proton (red) and the neutron (blue) kinematic variables after applying the high missing momentum cuts. Shown are (a) the missing momentum, (b) the leading proton/neutron momentum, (c) the leading proton/neutron scattering angle, and (d) the angle between the struck nucleon and the momentum transfer vector. All distributions are normalized to unity such that only their shapes are to be compared. 
(a)

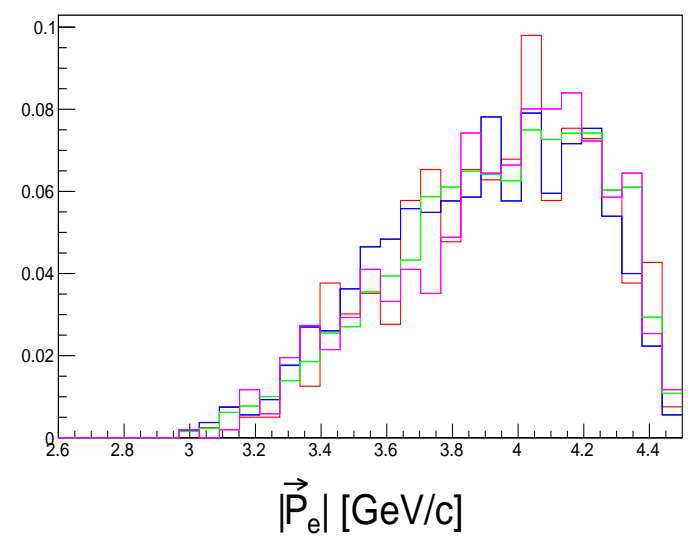

(c)

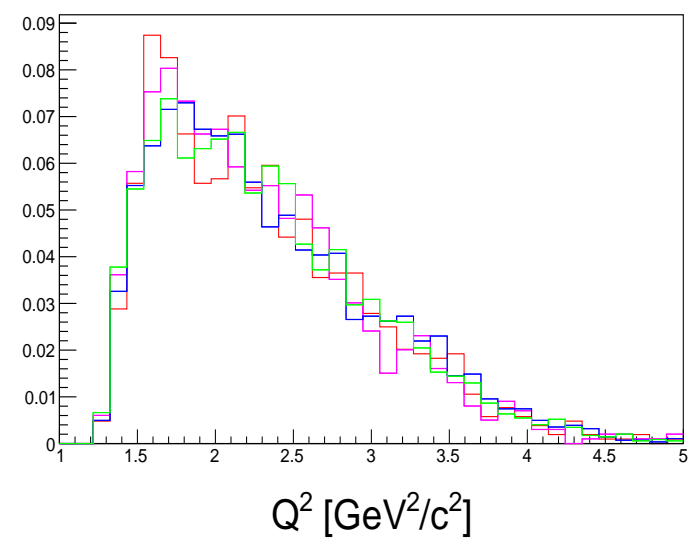

(b)

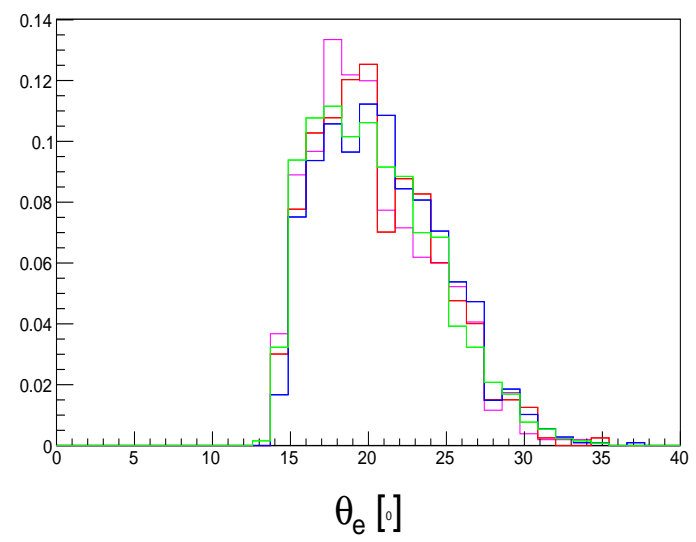

(d)

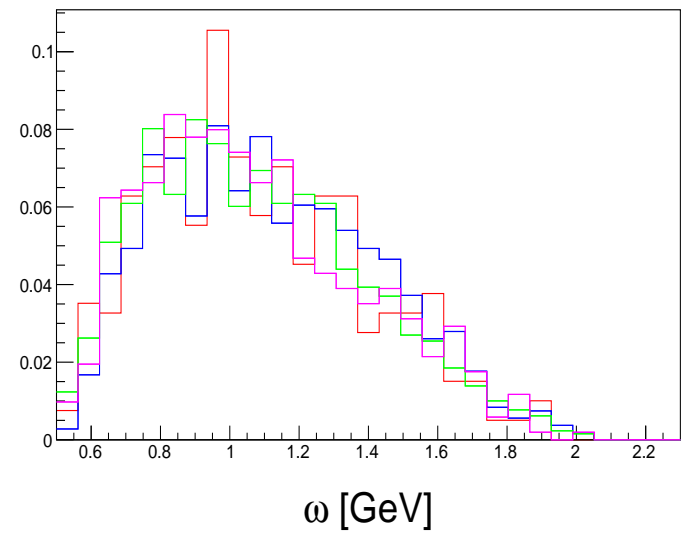

(e)

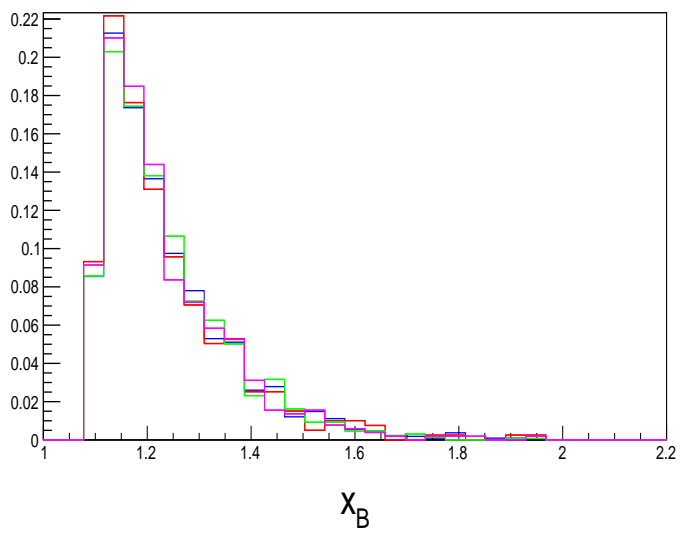

Figure 44: Same as Fig. 42 for the selected $\left(e, e^{\prime} n\right)$ events from C (blue), Al (red), Fe (green), and Pb (pink). 
(a)

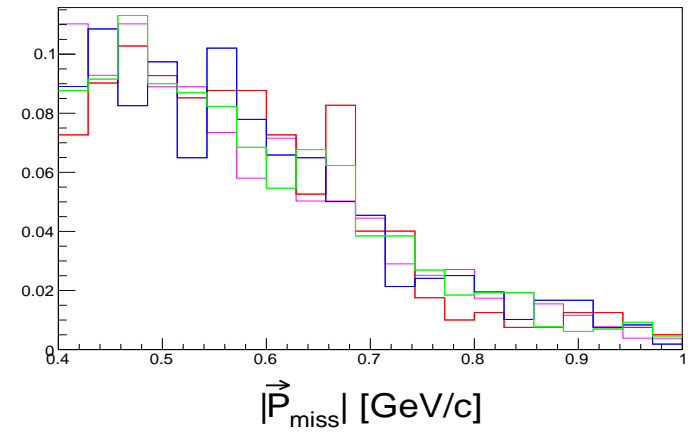

(c)

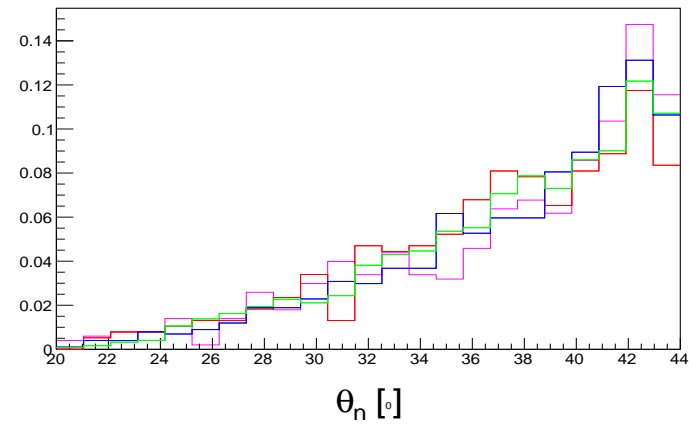

(b)

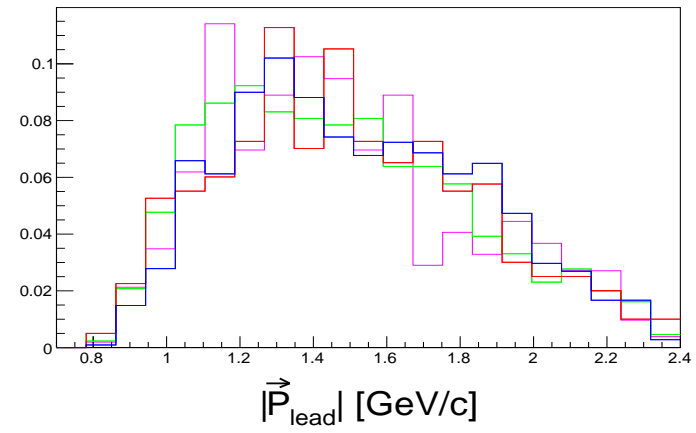

(d)

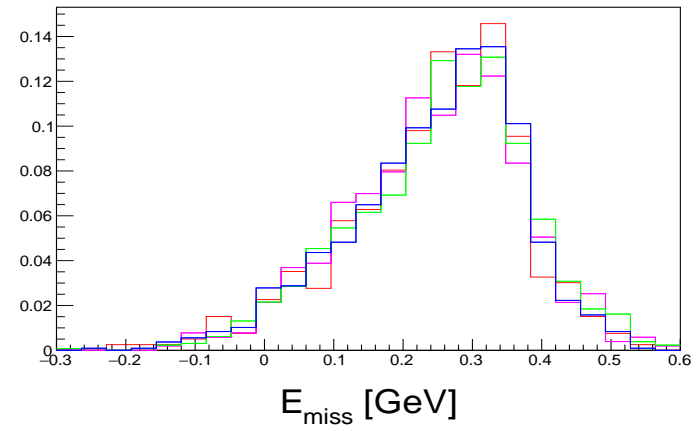

Figure 45: Same as Fig. 43 for $A\left(e, e^{\prime} n\right)$ events. Blue - C, red - Al, green - Fe, and pink $\mathrm{Pb}$.

\subsubsection{Identifying Low-Momentum (Mean-Field) events}

Low-momentum $\left(e, e^{\prime} N\right)(N=p / n)$ events, are QE events with the electron being scattered off a nucleon with low-momentum $\left(k<k_{F}\right)$, and low separation energy $(<\sim 80 \mathrm{MeV}) 1618$. Assuming the PWIA, as in Sec. 3.2.1 the initial momentum of the nucleon equals to the missing momentum $\left(\vec{p}_{\text {miss }}\right)$, and the separation energy to the missing energy of the knockout nucleon: $E_{\text {miss }}=\omega-T_{N}-T_{B}$, where $\omega$ is the energy transfer in the $\left(e, e^{\prime}\right)$ reaction, $T_{N}$ is the kinetic energy of the struck nucleon, and $T_{B}$ is the kinetic energy of the residual $A-1$ system. Figure 46 shows the missing energy vs. the missing momentum for $\left(e, e^{\prime} n\right)$ and $\left(e, e^{\prime} p\right)$ events. The latter are shown before and after momentum smearing. A QE peak is clearly observed for the un-smeared $\left(e, e^{\prime} p\right)$ events at $p_{\text {miss }}<250 \mathrm{MeV} / \mathrm{c}$ and $E_{\text {miss }}<80 \mathrm{MeV}$, but is not visible for neutrons and smeared protons due to the poor momentum resolution. Therefore, for low- $p_{\text {miss }}$ events, we used again the smeared protons to optimize the selection cuts, to minimize the false-positive and false-negative probabilities. 

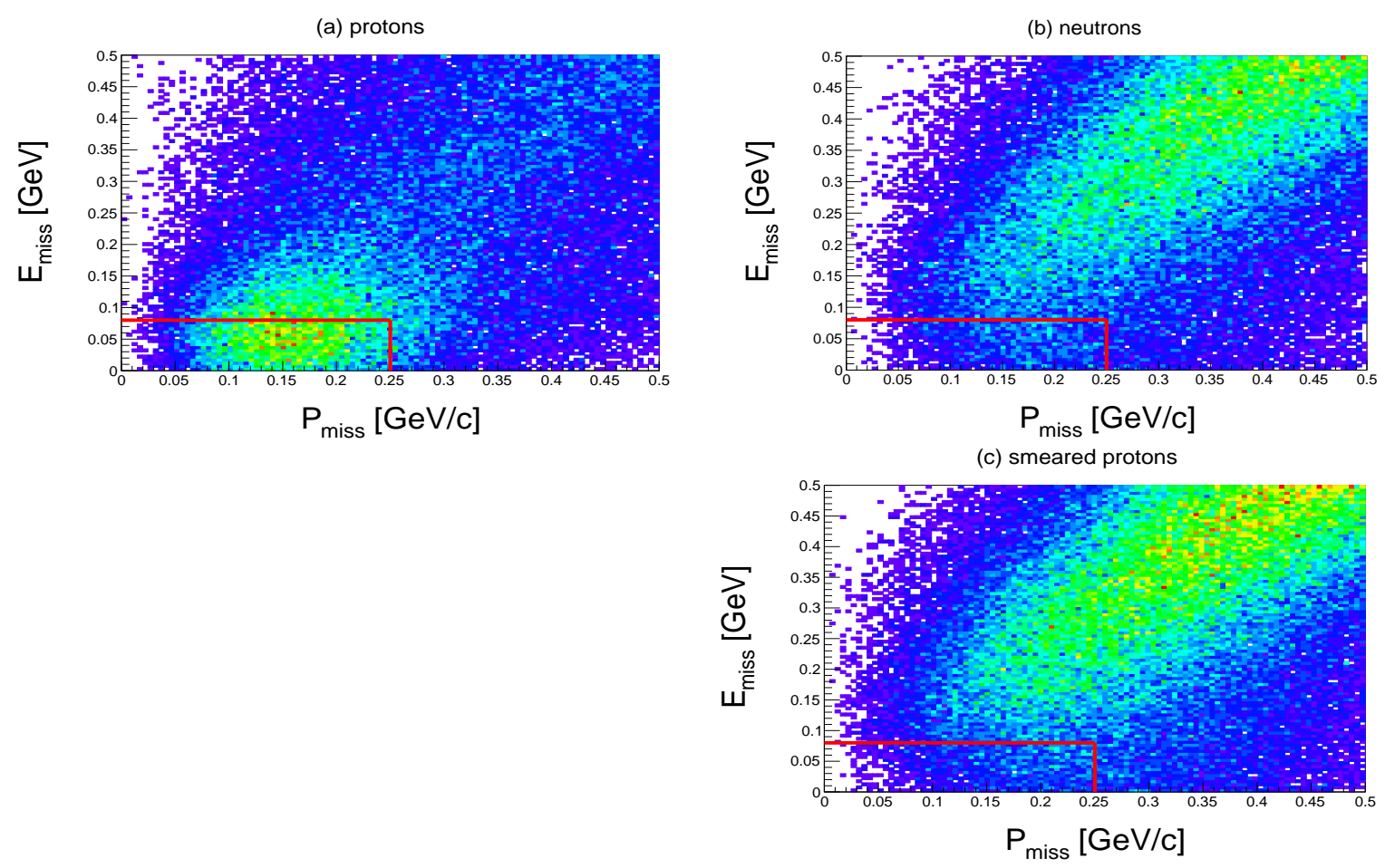

Figure 46: The missing energy vs. the missing momentum for (a) $\left(e, e^{\prime} p\right)$ events, (b) $\left(e, e^{\prime} n\right)$ events and (c) $\left(e, e^{\prime} p_{\text {smeared }}\right)$ events. The red lines represent the $\mathrm{QE}$ region.

Before optimizing the cuts to select low missing momentum and energy events, we first enhanced the MF contribution to the event sample by cutting on quantities that are insensitive to the poor neutron momentum resolution, namely the electron kinematic variables and the detected nucleon angle.

From the electron variables we extracted the energy transfer, $\omega$, the four-momentum transfer, $Q^{2}$, and $y$, a scaling variable related to the minimum initial momentum of the knocked-out nucleon in the direction of the momentum transfer:

$$
y \equiv\left[\left(M_{A}+\omega\right) \sqrt{\Lambda^{2}-M_{A-1}^{2} W^{2}}-|\vec{q}| \Lambda\right] / W^{2}
$$

with

$$
W=\sqrt{\left(M_{A}+\omega\right)^{2}-|\vec{q}|^{2}}, \Lambda=\left(M_{A-1}^{2}-M_{N}^{2}+W^{2}\right) / 2
$$

where $M_{A}, M_{A-1}$ and $M_{N}$ are, respectively, the masses of the target nucleus, residual nucleus, and nucleon.

Figure 47 shows the correlations between $y$ and $\omega$, and between $Q^{2}$ and $\theta_{N q}$, the angle between the detected nucleon and the momentum transfer vector. The distributions are shown for all events and for low- $p_{\text {miss }}$ and $E_{\text {miss }}$ events (i.e., $p_{\text {miss }}<250 \mathrm{MeV} / \mathrm{c}$ and $E_{\text {miss }}<80 \mathrm{MeV}$ for un-smeared protons). The low missing momentum and energy events populate very narrow parts of the phasespace. We therefore cut on $y, \omega$, and $\theta_{N q}$ based on the distributions of the un-smeared protons 
with low missing momentum and energy. The cuts are shown by the red boxes in Fig. 47 .

$$
\begin{gathered}
-0.05<y<0.25 \\
0.95<\omega<1.7 \mathrm{GeV} \\
\theta_{p q}<8^{\circ}
\end{gathered}
$$

The $Q^{2}$ cut shown in Fig. 47 (b) $\left(1.3<Q^{2}<3.5 \mathrm{GeV}^{2} / \mathrm{c}^{2}\right)$ was not directly implemented as cutting on $y$ and $\omega$ limit the selected events within this cut limits. It should be noted that for MF QE events, $y$ is expected to be centered around $y=0$. This is not the case here (see Fig. 47 (a)). The reason for this is the bias caused by the angular region we are probing, that is limited by the EC angular coverage (see Ref. 15 for details).

(a)

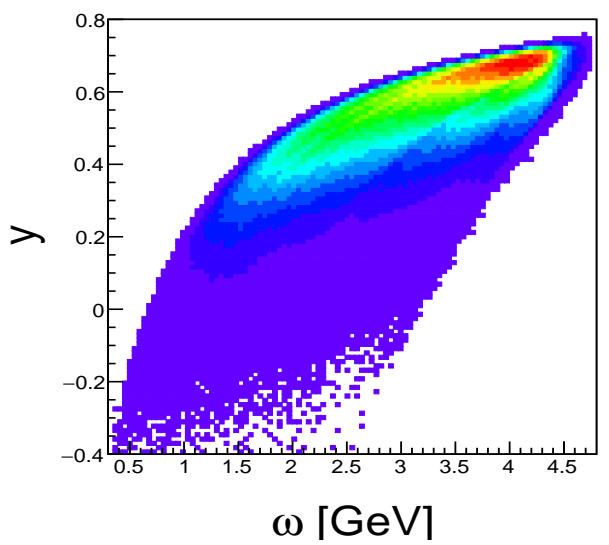

(b)

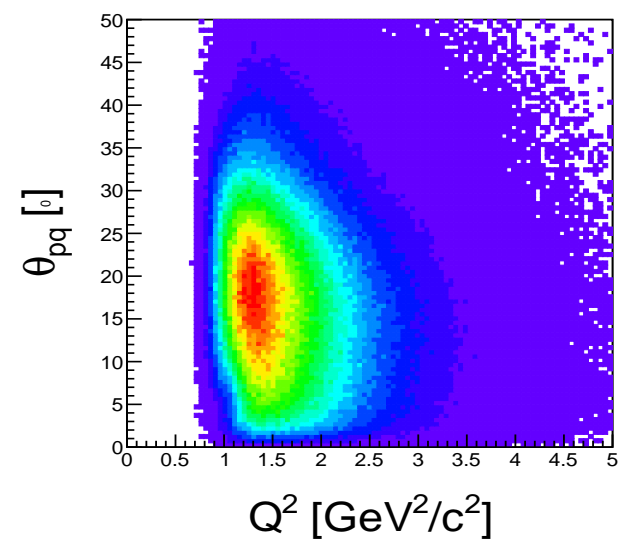

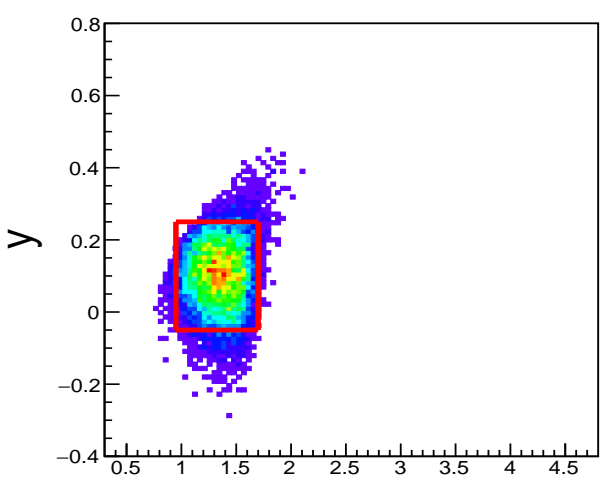

$\omega[\mathrm{GeV}]$

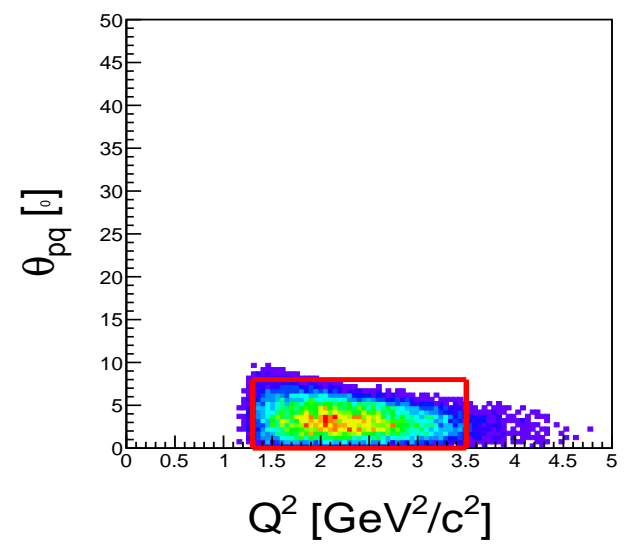

Figure 47: (a) The $y$ scaling variable vs. $\omega$ with (right) and without (left) the $p_{m i s s} \& E_{m i s s}$ QE cuts for un-smeared protons. (b) Same as (a) for $\theta_{p q}$ vs. $Q^{2}$.

Next, to determine the cuts on $E_{\text {miss }}$ and $p_{\text {miss }}$, we used the smeared protons in a similar way as described in Sec. 3.2.1 for the high-momentum event selection. We chose to optimize the cuts compared to un-smeared missing energy and momentum of $p_{\text {miss }}<250 \mathrm{MeV} / \mathrm{c}, E_{\text {miss }}<80+\epsilon(A)$ $\mathrm{MeV}$, where $\epsilon(A)$ term takes into account the difference in the average separation energy for nucleus A relative to C. Using many-body spectral functions from [19], we estimated the increased 
missing energy cuts for the other nuclei: $\epsilon(A)=0,5$, and $10 \mathrm{MeV}$ for ${ }^{27} \mathrm{Al},{ }^{56} \mathrm{Fe}$, and ${ }^{208} \mathrm{~Pb}$, respectively. Further, we applied different cut combinations and examined the resulting false positive and negative probabilities (see Fig. 48). Based on these rated, we selected the following cuts for neutrons:

$$
p_{\text {miss }}<300 \mathrm{MeV} / \mathrm{c} \quad E_{\text {miss }}<190 \mathrm{MeV}
$$

These cuts are in addition to the cuts listed in Eq. 11 above, and lead to false positive and negative probabilities of about $10 \%$ and $15 \%$ respectively.

Figure 49 and 50 show the kinematical distributions of the resulting ${ }^{12} \mathrm{C}\left(e, e^{\prime} n\right)$ and ${ }^{12} \mathrm{C}\left(e, e^{\prime} p\right)$ events. Figures 51 and 52 show the same distributions for $\left(e, e^{\prime} n\right)$ scattering events for all targets.
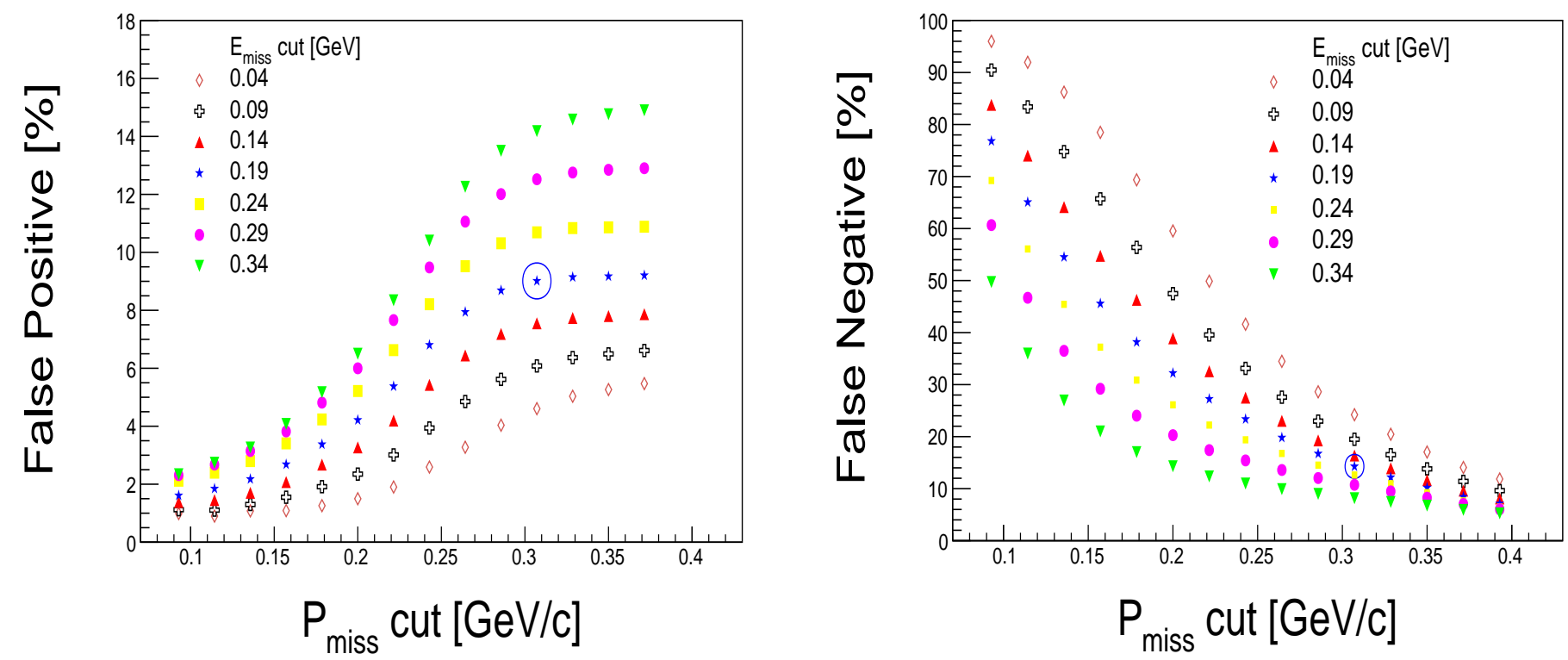

Figure 48: The false positive (left) and negative (right) probabilities for the low missing momentum (QE) events, as a function of the missing momentum cut for different missing energy cuts. The blue circle represents the selected cuts. See text for details. 
(a)

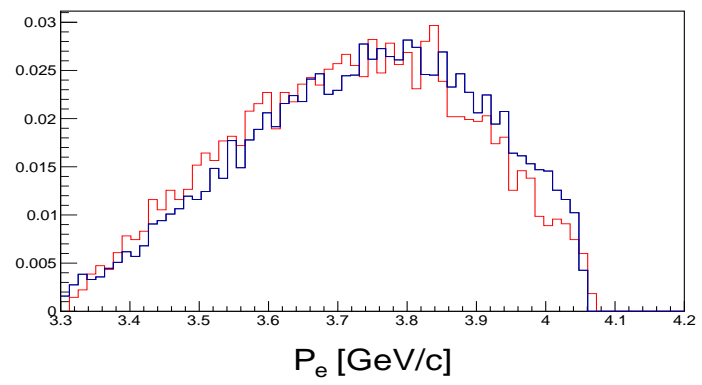

(c)

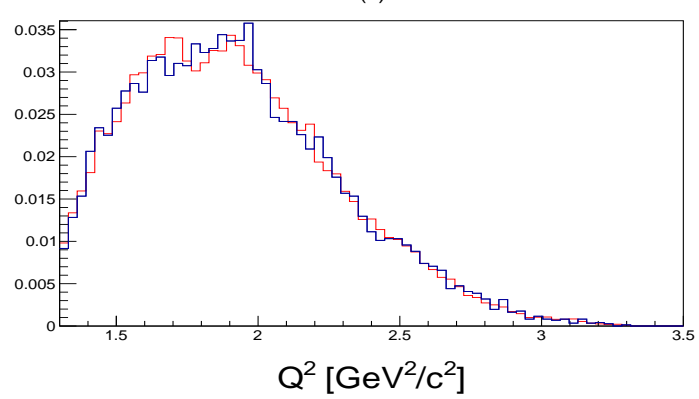

(e)

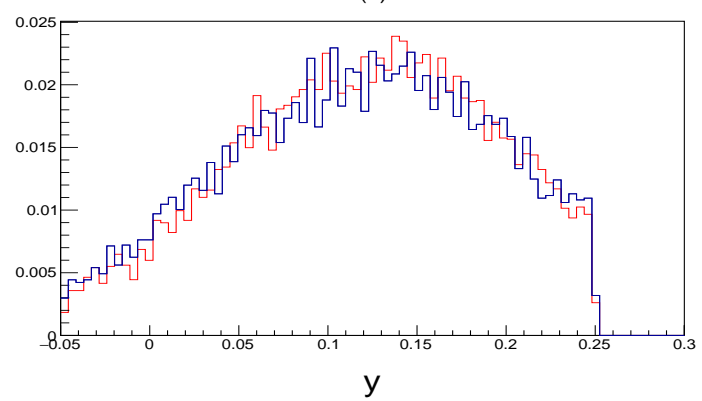

(b)

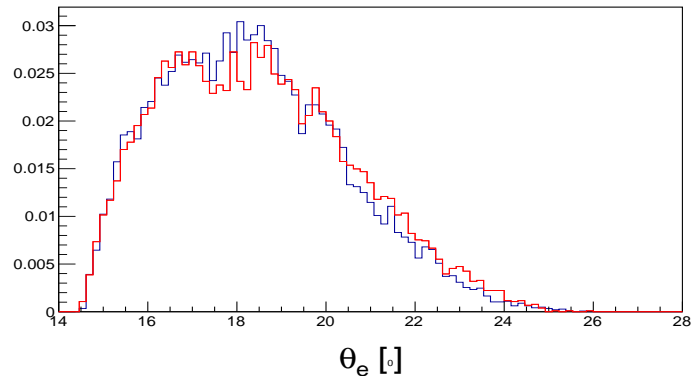

(d)

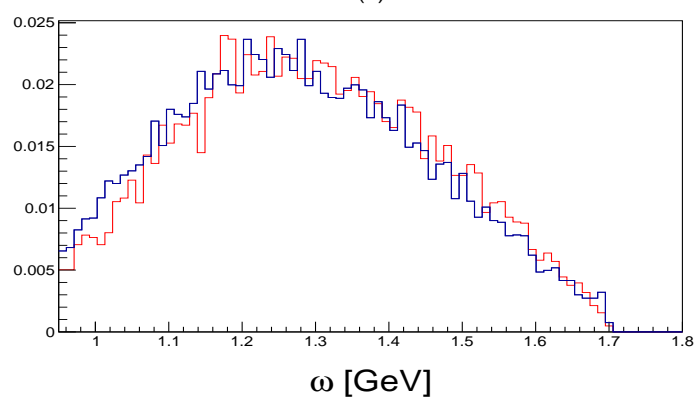

(f)

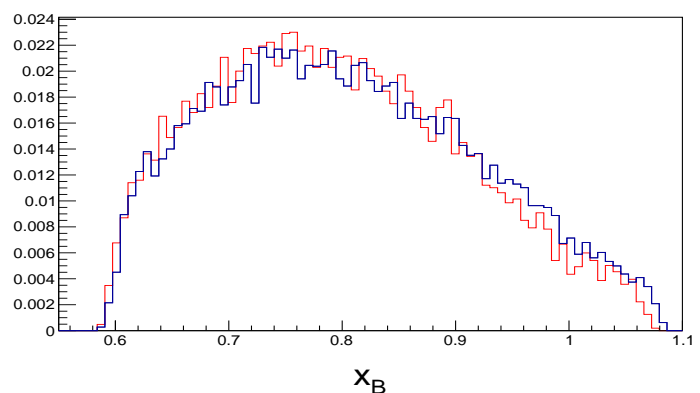

Figure 49: The electron kinematic variables for ${ }^{12} \mathrm{C}\left(e, e^{\prime} p_{\text {smeared }}\right)$ (red) and ${ }^{12} \mathrm{C}\left(e, e^{\prime} n\right)$ (blue) after applying the QE cuts: (a) the electron momentum, (b) the electron scattering angle, (c) $Q^{2}$, (d) $\omega$, (e) $y$, and (f) $x_{B}$. All distributions are normalized to unity such that only their shape is compared. 

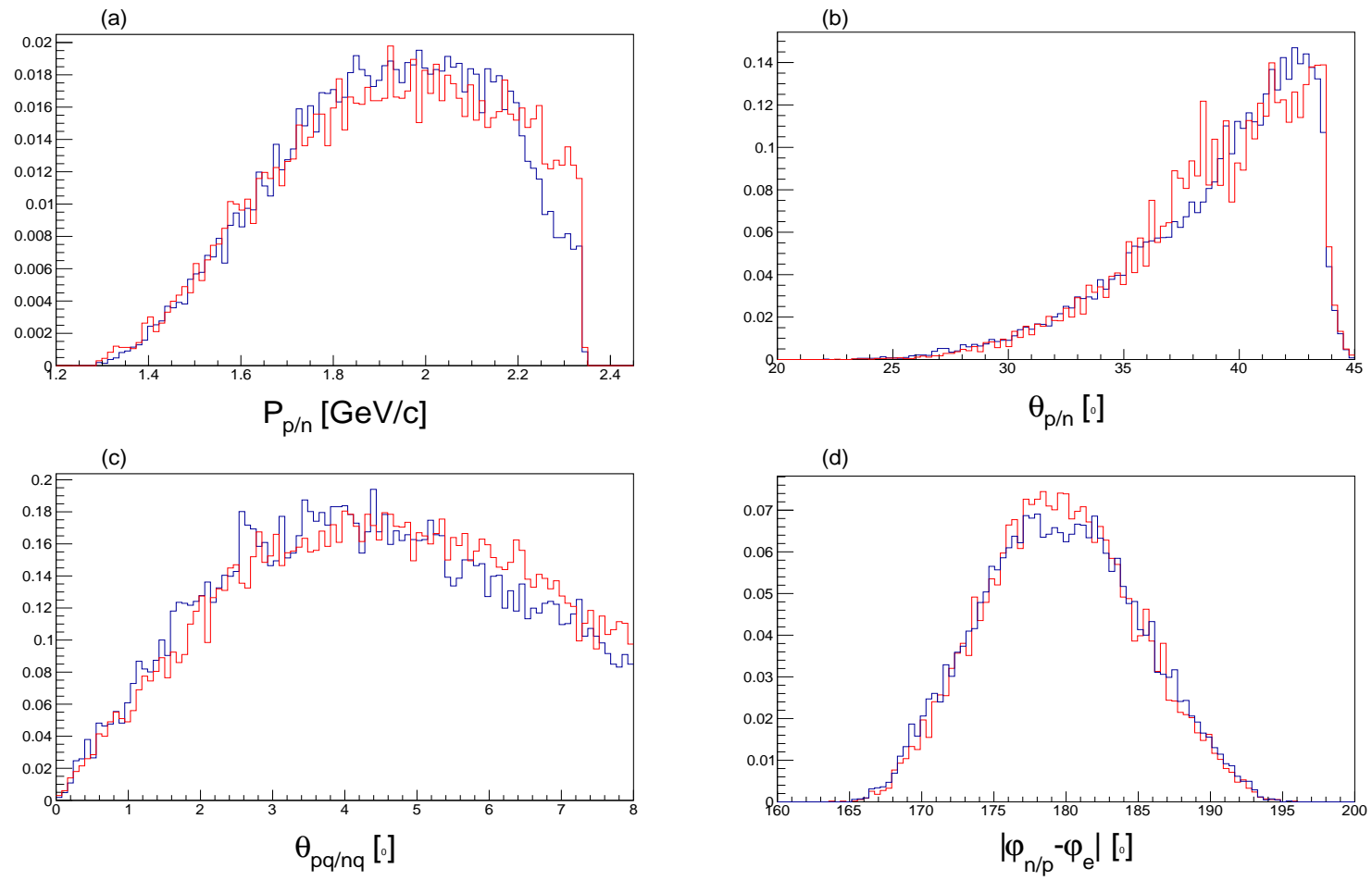

Figure 50: The smeared protons (red) and neutrons (blue) quantities after applying the QE cuts: (a) nucleon momentum, (b) scattering angle, (c) the angle between the nucleon and the momentum transfer vector, and (d) the absolute difference between the out of plane scattering angles of the nucleon and the electron. All distributions are normalized to unity such that only their shape is compared. 

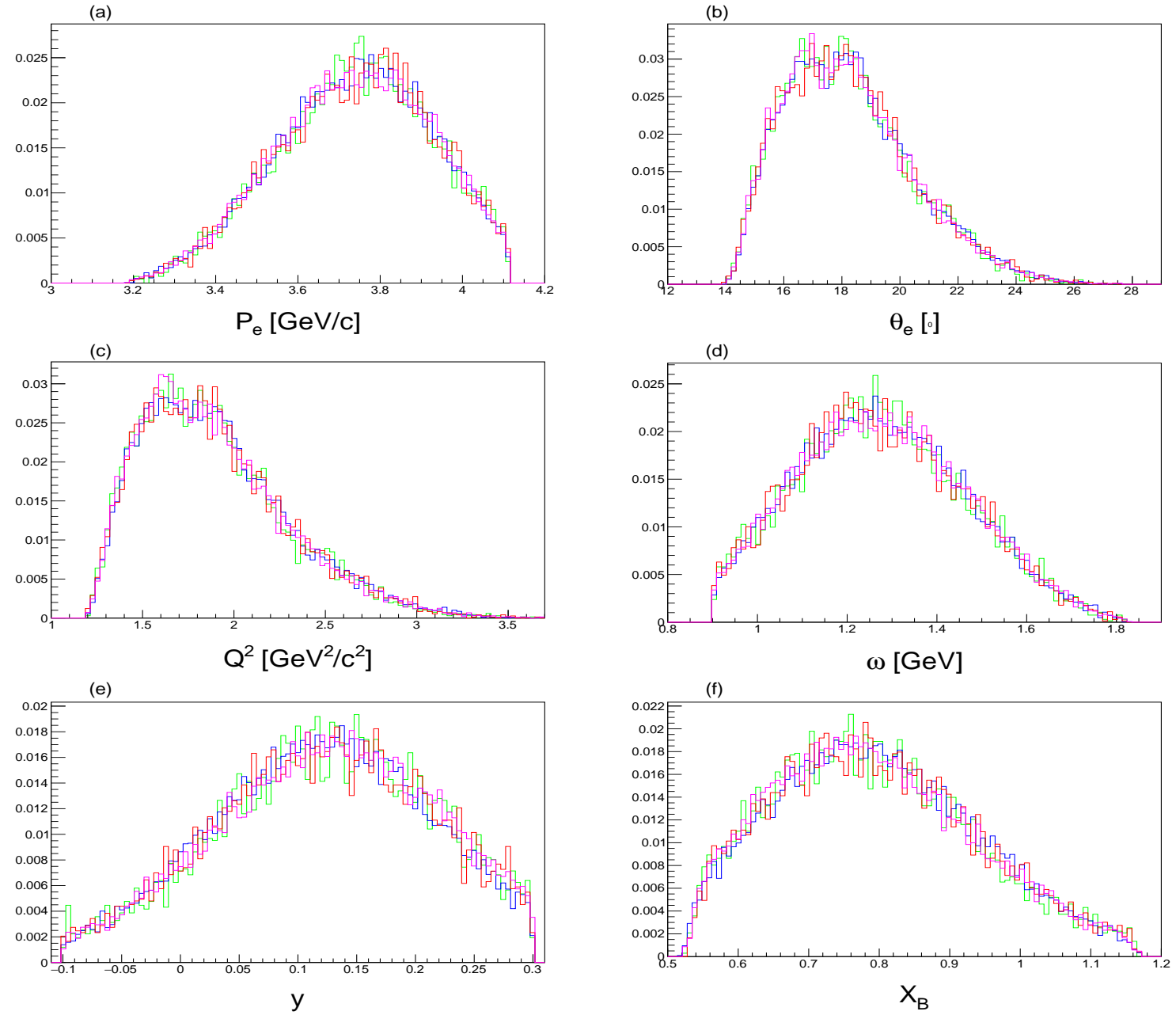

Figure 51: Same as Fig. 49 for $A\left(e, e^{\prime} n\right)$ events after applying the QE cuts. Blue - C, red - Al, green - Fe, and pink $-\mathrm{Pb}$. 

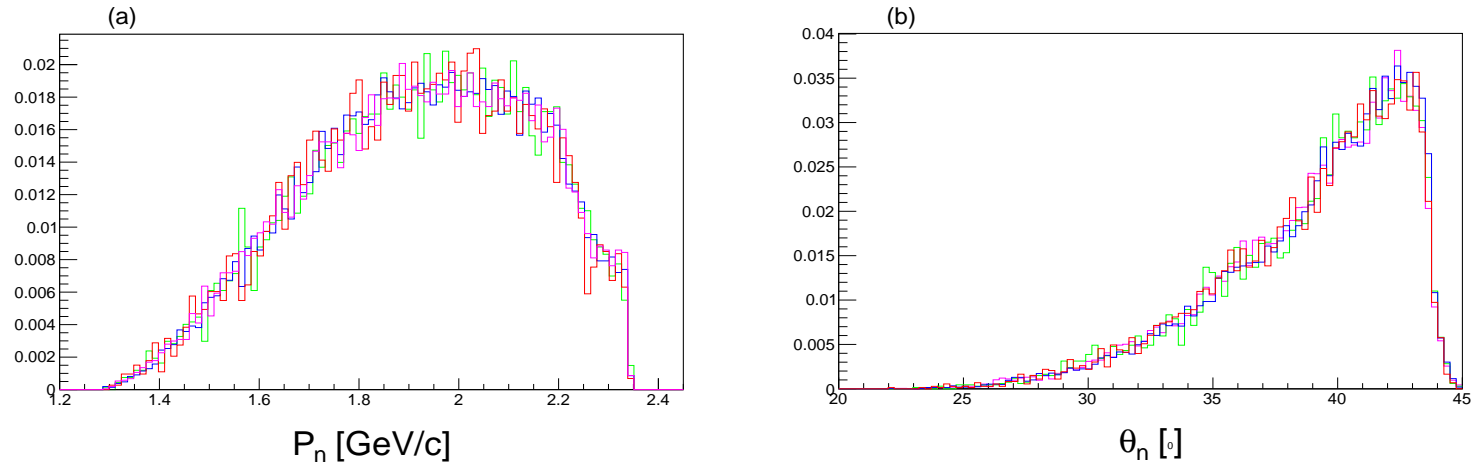

(c)
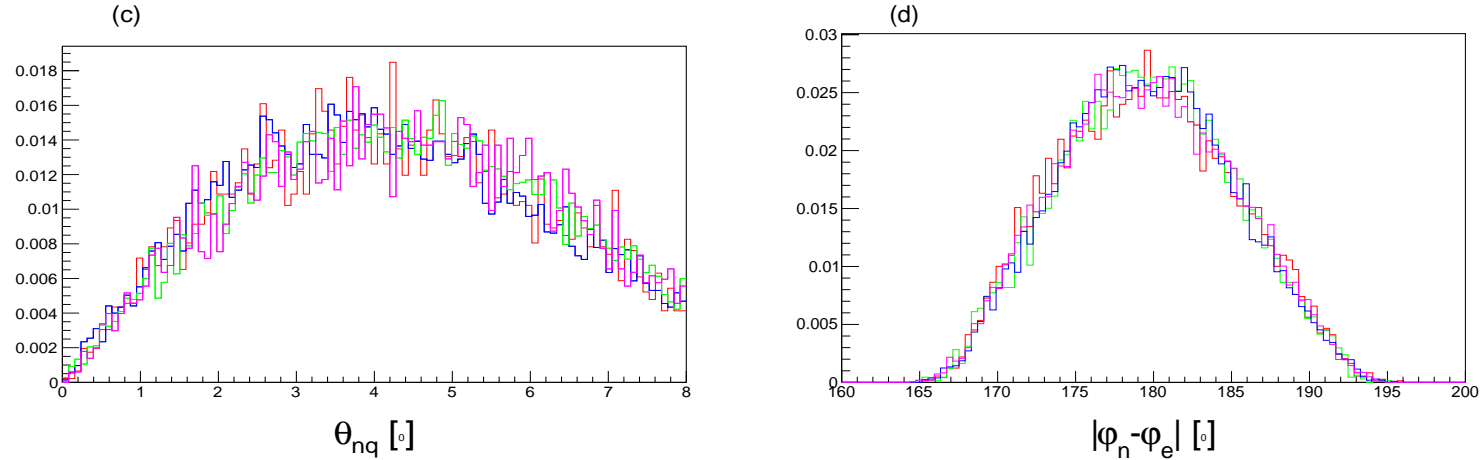

Figure 52: Same as Fig. 50 for $A\left(e, e^{\prime} n\right)$ events after applying the QE cuts. Blue - C, red - Al, green - Fe, and pink - $\mathrm{Pb}$.

\subsection{Identifying the $A\left(e, e^{\prime} n p\right)$ and $A\left(e, e^{\prime} p p\right)$ High-Momentum Events}

After identifying high-momentum $A\left(e, e^{\prime} n\right)$ and $A\left(e, e^{\prime} p\right)$ we moved to an exclusive triple coincidence $A\left(e, e^{\prime} n p\right)$ and $A\left(e, e^{\prime} p p\right)$ measurements. We started with the sample of $A\left(e, e^{\prime} n\right)$ and $A\left(e, e^{\prime} p\right)$ selected in Sec. 3.2.1 and demand to have another proton detected in a coincidence. This proton which we refer to as "recoil proton" is required to have momentum $p_{\text {rec }}>350 \mathrm{MeV} / \mathrm{c}$.

Since the recoil protons have relatively low momenta, we corrected their momenta for energy loss in the target and the CLAS detector, following the procedure in Ref. 14. The correction factor was estimated using GSIM, CLAS Geant-3 Monte-Carlo simulation 20. The proton momentum was randomized uniformly, and for each simulated event, both the generated $\left(P_{G e n}\right)$ and reconstructed $\left(P_{R e c}\right)$ momentum of the proton were saved. Figure 53 shows the difference between the generated and reconstructed energy as a function of the proton reconstructed momentum. A momentum correction function was fitted to the mean energy loss, which resulted in:

$$
d E=0.0013+\frac{0.00084}{\left(0.074+P_{\text {rec }}\right)^{2}}
$$




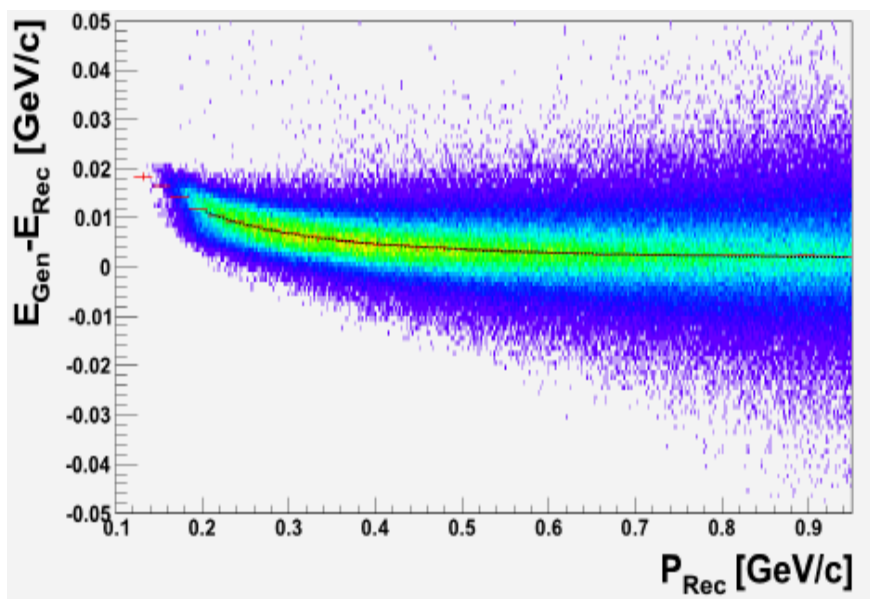

Figure 53: The difference between the energy of generated and reconstructed $\left(E_{G e n}-E_{R e c}\right)$ protons as a function of the reconstructed proton momentum $\left(p_{R e c}\right)$, extracted using GSIM [20]. The red crosses are the mean values of each $\left(p_{R e c}\right)$ bin. The dashed line represents the correction that needs to be applied to the reconstructed momentum. This figure was adapted from [14].

Figure 54 shows the energy deposited in the TOF counters for the recoil protons vs. their momentum for the different targets. In order to eliminate pions contamination, we chose to cut on SC energy deposit $>15 \mathrm{MeV}$. 
$\left({ }^{12} \mathrm{C}\right)$

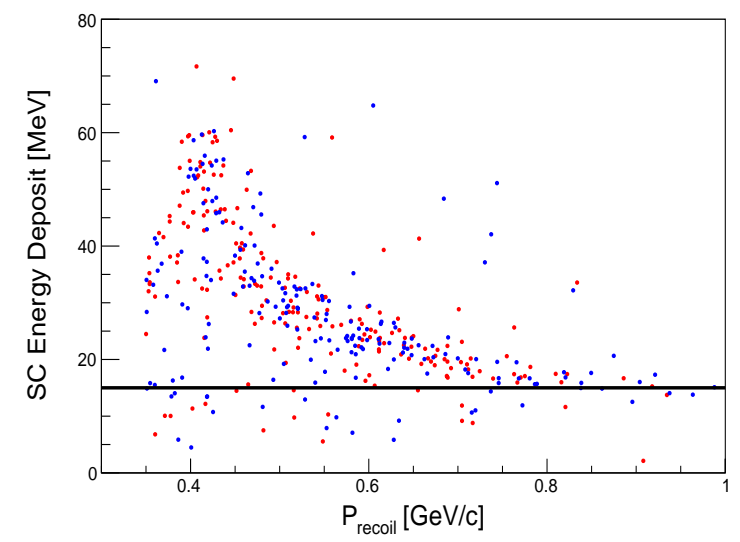

$\left({ }^{56} \mathrm{Fe}\right)$

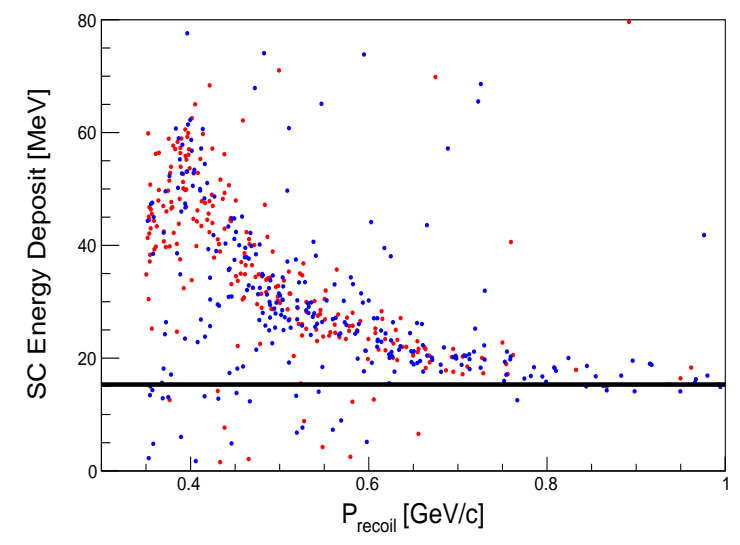

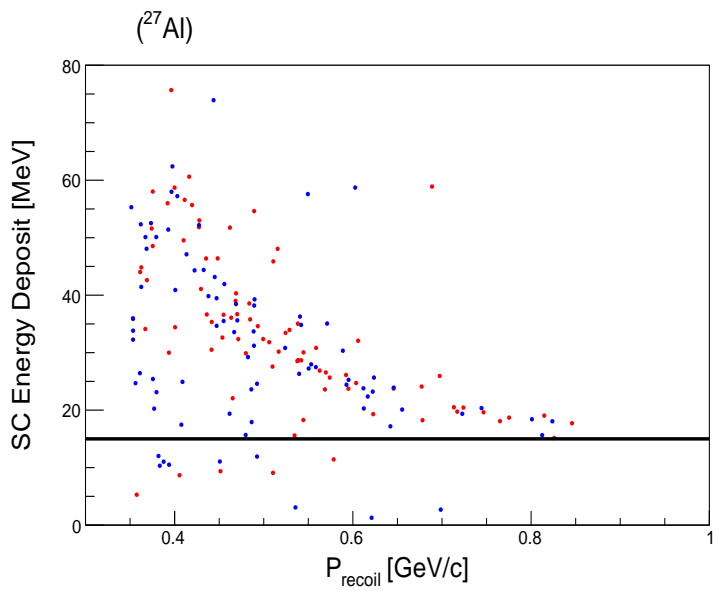

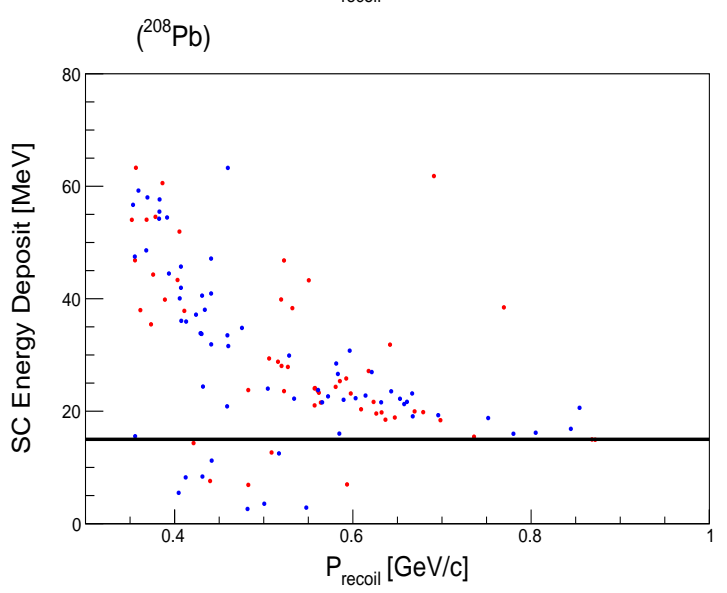

Figure 54: Energy deposited in the TOF counters for the recoil protons vs. their momentum for the selected smeared $\left(e, e^{\prime} p p\right)$ (red) and $\left(e, e^{\prime} n p\right)$ (blue) events. The black line shows the cut applied to eliminate pions contamination.

Figures 5557 show the kinematical distributions of the resulting ${ }^{12} \mathrm{C}\left(e, e^{\prime} n p\right)$ and ${ }^{12} \mathrm{C}\left(e, e^{\prime} p p\right)$ events. Note that due to SRC kinematics the recoil protons are expected to show correlation with $\vec{p}_{m i s s}$ of the $\left(e, e^{\prime} N\right)$ reaction. That correlation can not be explained by simple phase-space consideration. In Fig. 57, we compare the measured distributions with these of a random sample of $\left(e, e^{\prime} n p\right)$ and $\left(e, e^{\prime} p p\right)$. We define a random event as one in which the electron and the leading nucleon are both taken from one event, while the recoil proton is taken from another. As can be seen in the figure it is clear that the measured distributions are not produced due to detector effects. Figures 5860 show the same distributions for $\left(e, e^{\prime} n p\right)$ scattering events for all targets. 

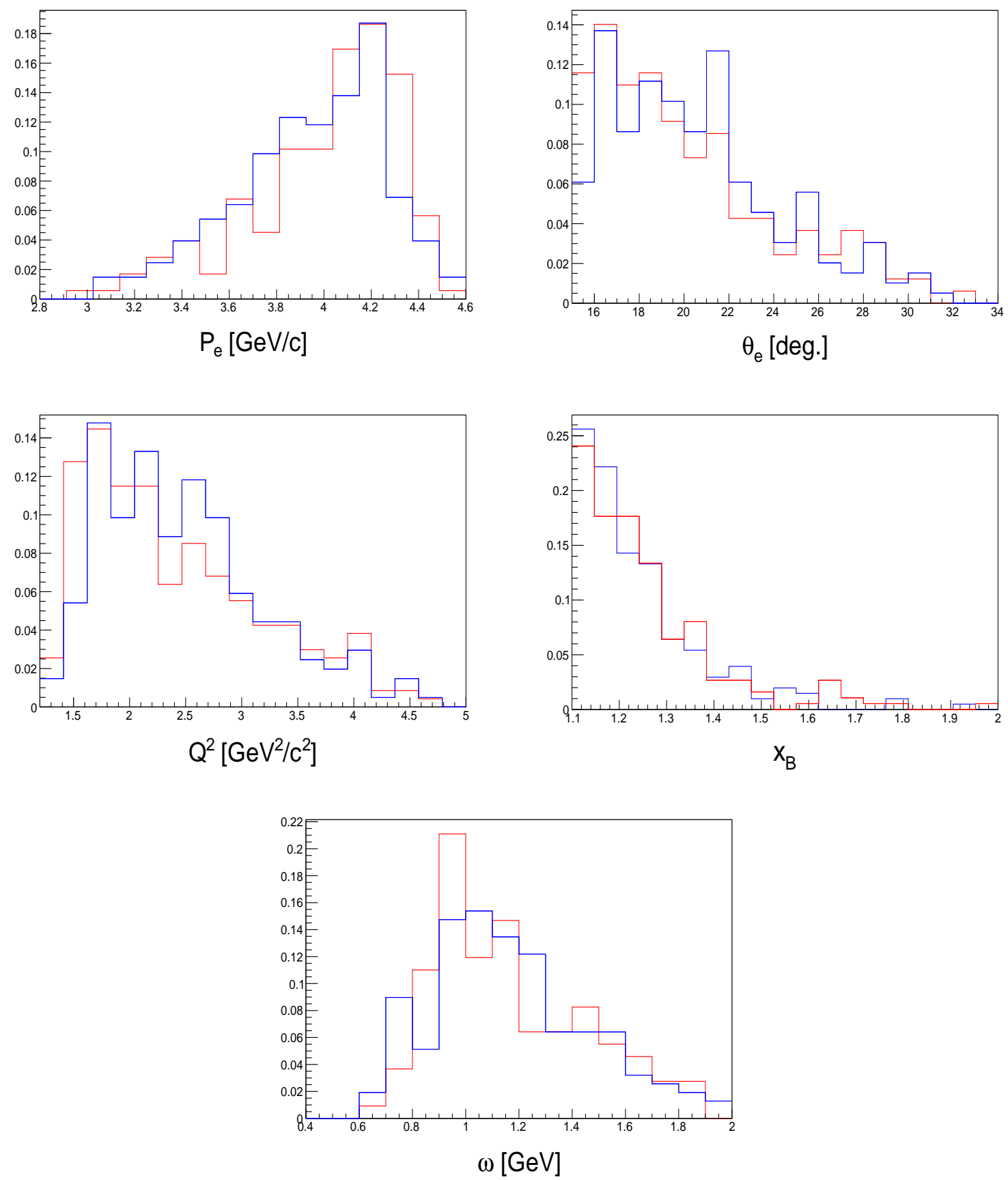

Figure 55: The electron kinematic variables for smeared ${ }^{12} \mathrm{C}\left(e, e^{\prime} p p\right)$ (red) and ${ }^{12} \mathrm{C}\left(e, e^{\prime} n p\right)$ (blue) selected events. Shown are the electron momentum, the electron scattering angle, $Q^{2}, x_{B}$, and $\omega$. All distributions are normalized to unity such that only their shapes are to be compared. 

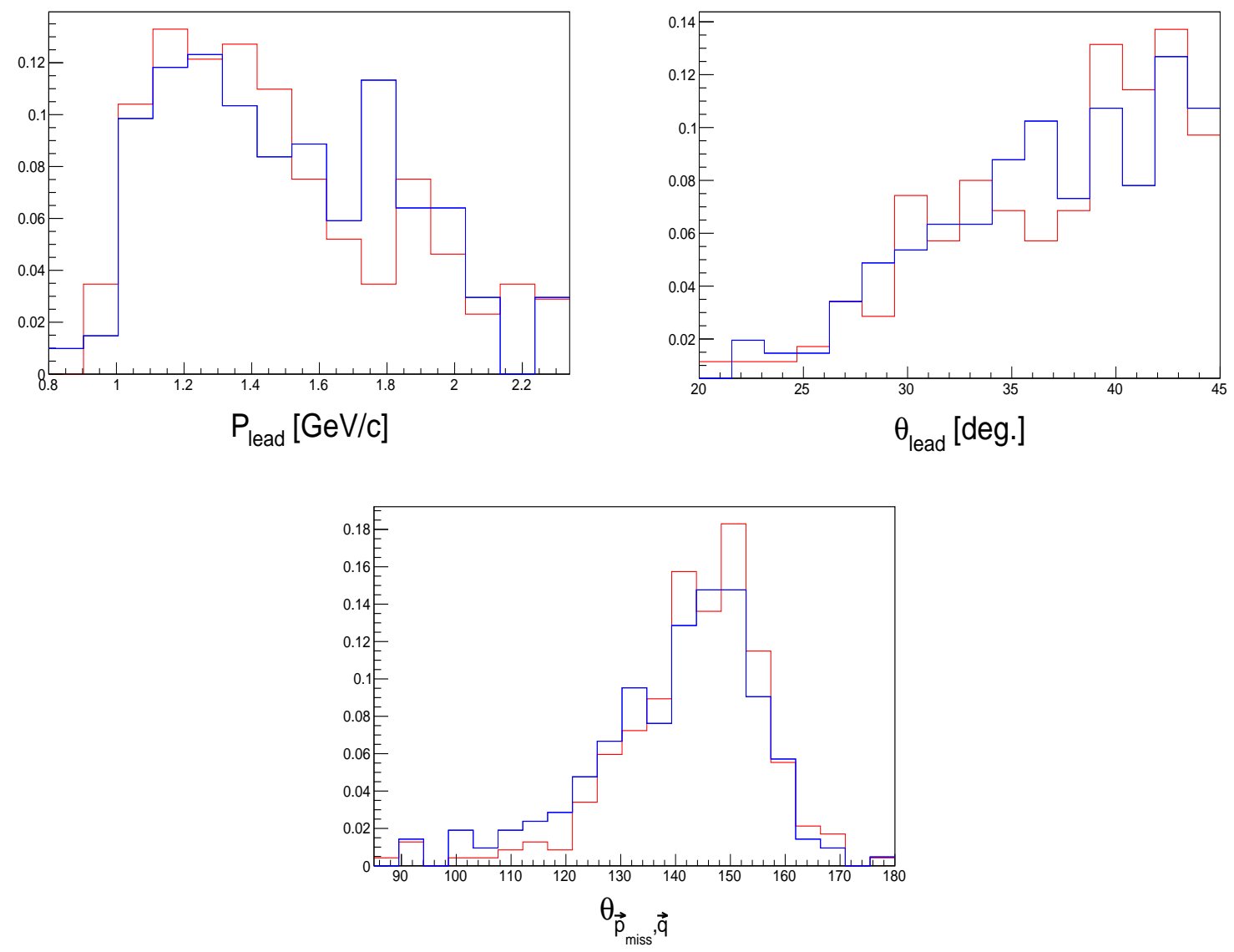

Figure 56: Same as Fig. 55 for the proton/neutron kinematic variables. Shown are the nucleon momentum, the nucleon scattering angle, and the angle between the initial nucleon momentum $p_{\text {miss }}$ and the $q$ vector. All distributions are normalized to unity such that only their shapes are to be compared. 

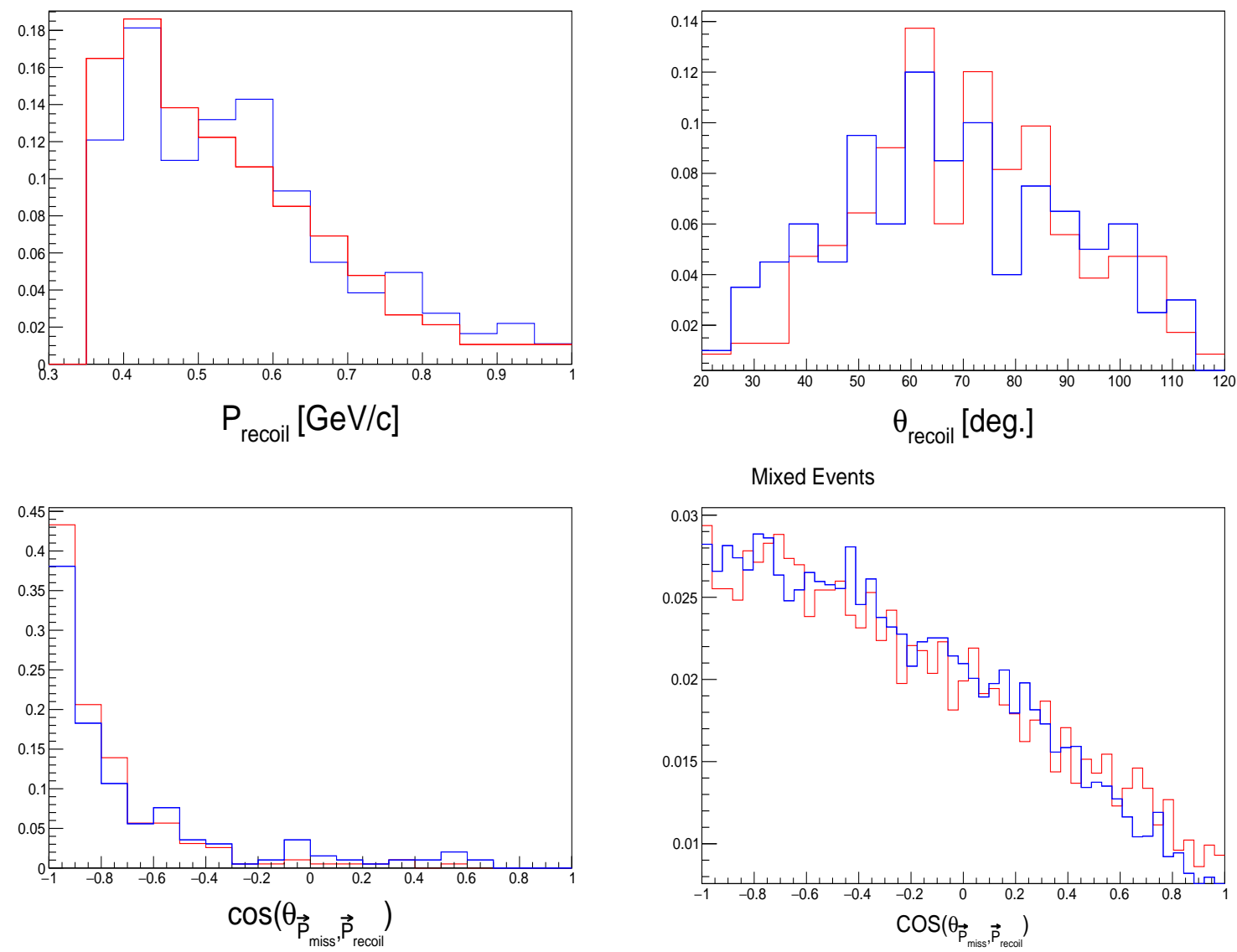

Figure 57: Same as Fig. 55 for the recoil proton kinematic variables. Shown are its momentum, its scattering angle, and the opening angle between the leading nucleon and the recoil proton for the $n p$ and $p p$ samples. Also shown (bottom right) the opening angle for random samples obtained from mixed events (see text for details). All distributions are normalized to unity such that only their shapes are to be compared. 

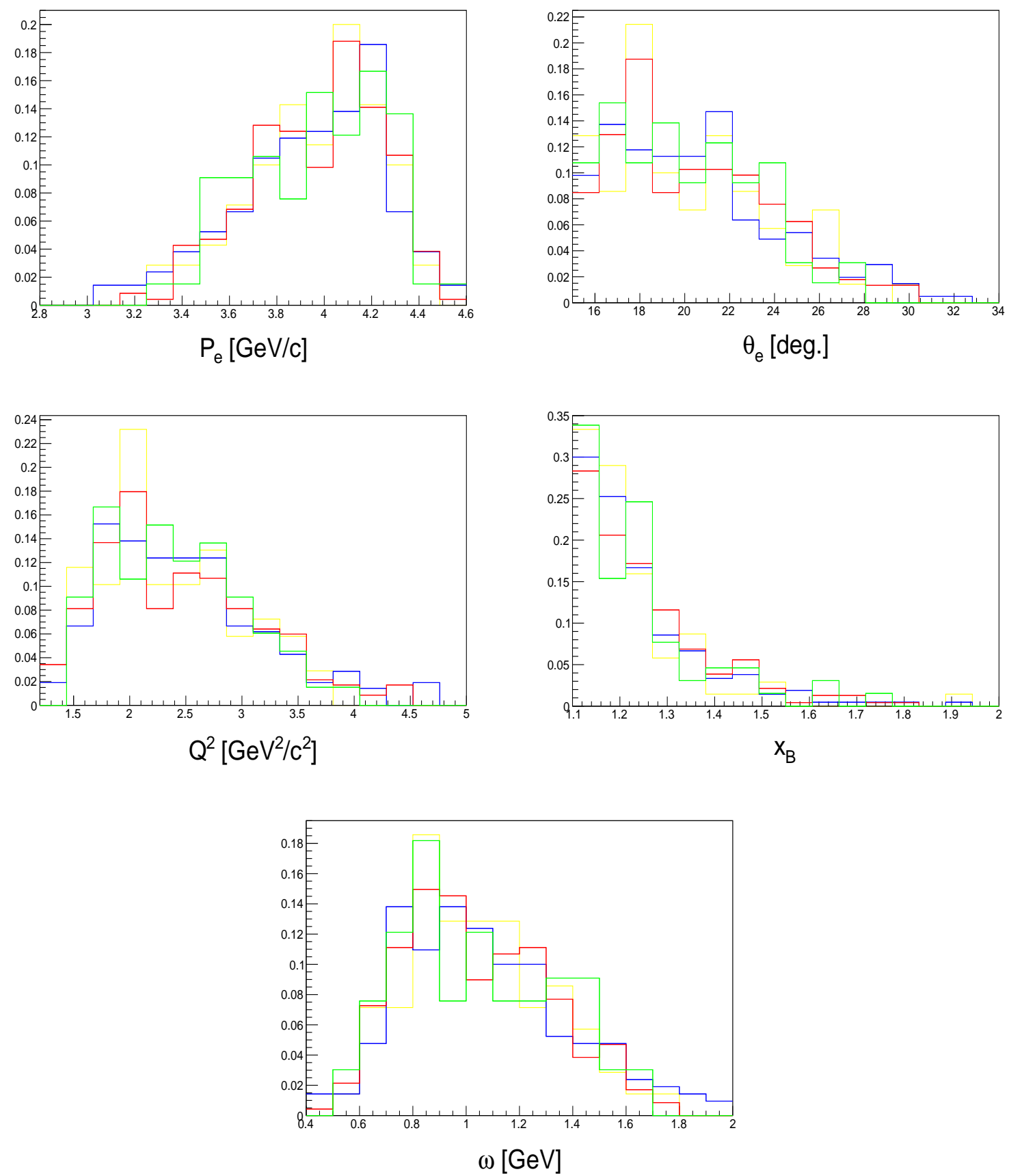

Figure 58: Same as Fig. 55 for $A\left(e, e^{\prime} n p\right)$ events. Blue - C, yellow - Al, red - Fe, and green - Pb. 

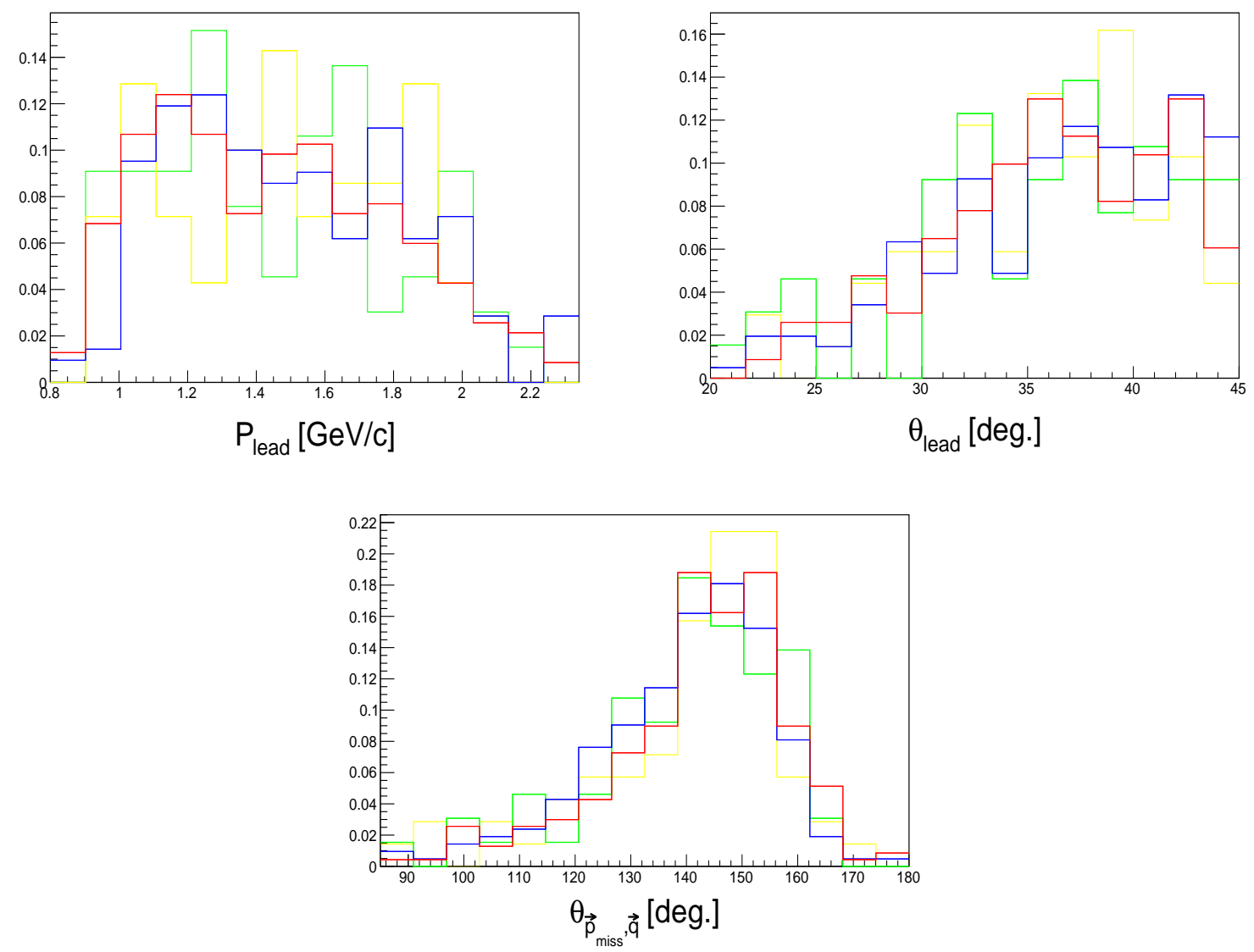

Figure 59: Same as Fig. 56 for $A\left(e, e^{\prime} n p\right)$ events. Blue - C, yellow - Al, red - Fe, and green - Pb. 

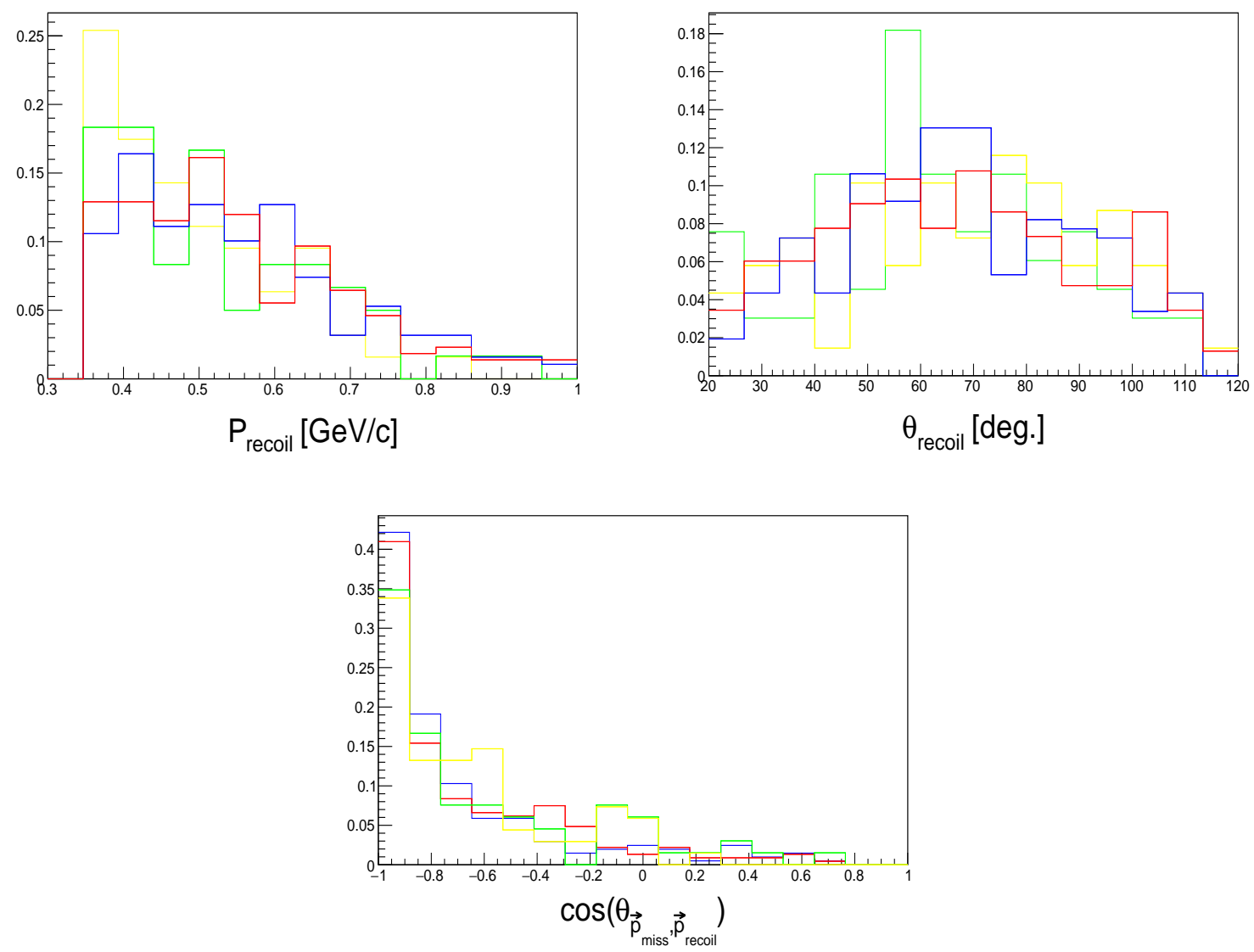

Figure 60: Same as Fig. 57 for $A\left(e, e^{\prime} n p\right)$ events. Blue - C, yellow - Al, red - Fe, and green - Pb. 


\section{Results}

\subsection{Extracting $\frac{A\left(e, e^{\prime} N\right)}{C\left(e, e^{\prime} N\right)}$ cross-section ratios}

$\frac{A\left(e, e^{\prime} p\right)}{C\left(e, e^{\prime} p\right)}$ ratios

To test the selection cuts developed for the smeared proton sample, we started by extracting the $\frac{A\left(e, e^{\prime} p\right)}{C\left(e, e^{\prime} p\right)}$ cross-section ratios for both smeared and un-smeared protons in both the mean-field (low- $p_{\text {miss }}$ ) and the SRC (high- $p_{\text {miss }}$ ) kinematics discussed in Sec. 3.2. The extraction of the cross-section ratios from the measured event yields requires several corrections:

- Acceptance correction - Since all solid targets were held in the same position with regard to the CLAS spectrometer, the kinematics of reconstructed events from all taget nuclei are the same. Therefore, CLAS acceptance effects and the electron reconstruction efficiency cancel in the $A\left(e, e^{\prime} p\right) / C\left(e, e^{\prime} p\right)$ cross-section ratios.

- Radiative correction - Due to the large acceptance of CLAS, radiative effects affect mainly the electron kinematics. They also largely cancel in the cross-section ratios. We corrected for this using the calculations of [23, which are commonly used by previous analyses [6]24]. The calculations are based on the virtual-nucleon impulse approximation of $\left(e, e^{\prime}\right)$ inclusive processes.

- Normalization corrections - The measured number of events were weighted by the integrated luminosity for each target. The luminosity is the product of the target areal density and the accumulated beam charge for each target. The target areal densities were measured before the experiment and are listed in Table 3 . The integrated beam charge was measured by a Faraday Cup located downstream of the beamline, and was summed for all runs on each target.

- False positive and negative - Table 8 lists the resulting false positive and negative probabilities for each target and kinematics. The false positive and negative corrections are about $10 \%$ to $15 \%$ and vary in opposite directions. They therefore largely cancel each other leading to less than $5 \%$ correction.

\begin{tabular}{|c|c|c|}
\hline Nucleus & False positive [\%] & False negative [\%] \\
\hline \hline $\mathrm{C}$ & 9.4 & 15.3 \\
\hline $\mathrm{Al}$ & 9.6 & 15.1 \\
\hline $\mathrm{Fe}$ & 10.0 & 15.0 \\
\hline $\mathrm{Pb}$ & 10.7 & 14.8 \\
\hline
\end{tabular}

\begin{tabular}{|c|c|c|}
\hline Nucleus & False positive [\%] & False negative [\%] \\
\hline \hline $\mathrm{C}$ & 17.1 & 16.2 \\
\hline $\mathrm{Al}$ & 16.9 & 16.0 \\
\hline $\mathrm{Fe}$ & 16.7 & 16.1 \\
\hline $\mathrm{Pb}$ & 17.6 & 16.4 \\
\hline
\end{tabular}

Table 8: The false positive and negative probabilities of all targets for low-momentum (left) and high-momentum (right) events. 
The contributions to the uncertainty in determing the requested ratios include:

\section{- Statistics}

- Sensitivity to the event selection cuts - Each event cut was modified over a given range and the resulting change in the relative event yield was taken as a systematic uncertainty. Tables 9 and 10 list the event selection cuts used in each kinematics and the range used for the sensitivity study, for smeared and un-smeared protons. Since the smeared protons are the ones to be compared with neutrons, changes in their relative event yield are presented in Table 11 .

- Radiative correction - A previous study 24 showed that this is a negligible correction to the $A / C$ ratios. Therefore, no contribution to the systematic uncertainties was assumed.

- Normalization correction - The uncertainty on the product of the accumulated beam charge and target thickness was estimated to be $2 \%$.

- False positive and negative - We assume the full value of this correction as a systematic uncertainty on the ratios.

\begin{tabular}{|c|c|}
\hline Cut (smeared protons) & Sensitivity range \\
\hline \hline$-0.05<y<0.25$ & \pm 0.05 \\
\hline $0.95<\omega<1.7 \mathrm{GeV}$ & $\pm 0.1 \mathrm{GeV}$ \\
\hline$\theta_{p q}<8^{\circ}$ & $\pm 1^{\circ}$ \\
\hline$p_{\text {miss }}<0.3 \mathrm{GeV} / \mathrm{c}$ & $\pm 0.025 \mathrm{GeV} / \mathrm{c}$ \\
\hline$E_{\text {miss }}<0.19 \mathrm{GeV}$ & $\pm 0.02 \mathrm{GeV}$ \\
\hline
\end{tabular}

\begin{tabular}{|c|c|}
\hline Cut (un-smeared protons) & Sensitivity range \\
\hline \hline$p_{\text {miss }}<0.25 \mathrm{GeV} / \mathrm{c}$ & $\pm 0.025 \mathrm{GeV} / \mathrm{c}$ \\
\hline$E_{\text {miss }}<0.08 \mathrm{GeV}$ & $\pm 0.008 \mathrm{GeV}$ \\
\hline
\end{tabular}

Table 9: The M.F. event-selection cuts, and the range used for the sensitivity study.

\begin{tabular}{|c|c|c|}
\hline Cut (smeared protons) & Cut (un-smeared protons) & Sensitivity range \\
\hline \hline$x_{B}>1.1$ & $x_{B}>1.2$ & \pm 0.05 \\
\hline${ }^{*} \theta_{p q}<25^{\circ}$ & ${ }^{*} \theta_{p q}<25^{\circ}$ & $\pm 5^{\circ}$ \\
\hline${ }^{*} 0.62<p_{p} / q<1.1$ & ${ }^{*} 0.62<p_{p} / q<0.96$ & \pm 0.05 \\
\hline$M_{\text {miss }}<1.175 \mathrm{GeV} / \mathrm{c}^{2}$ & $M_{\text {miss }}<1.1 \mathrm{GeV} / \mathrm{c}^{2}$ & $\pm 0.025 \mathrm{GeV} / \mathrm{c}^{2}$ \\
\hline $0.4<p_{\text {miss }}<1 \mathrm{GeV} / \mathrm{c}$ & $0.3<p_{\text {miss }}<1 \mathrm{GeV} / \mathrm{c}$ & $\pm 0.025 \mathrm{GeV} / \mathrm{c}$ \\
\hline
\end{tabular}

Table 10: The high-momentum event-selection cuts, and the range used for the sensitivity study. *The leading proton $\left(\theta_{p q}\right.$ and $\left.p_{p} / q\right)$ cuts were changed simultaneously.

\begin{tabular}{|c|c|c|c|}
\hline MF Cut & $\mathrm{Al} / \mathrm{C}$ & $\mathrm{Fe} / \mathrm{C}$ & $\mathrm{Pb} / \mathrm{C}$ \\
\hline \hline$-0.05<y<0.25$ & $1.6 \%$ & $1.3 \%$ & $1.2 \%$ \\
\hline $0.95<\omega<1.7 \mathrm{GeV}$ & $1.4 \%$ & $0.8 \%$ & $2.0 \%$ \\
\hline$\theta_{p q}<8^{\circ}$ & $1.3 \%$ & $1.9 \%$ & $1.6 \%$ \\
\hline$P_{\text {miss }}<0.3 \mathrm{GeV} / \mathrm{c}$ & $1.2 \%$ & $2.0 \%$ & $1.8 \%$ \\
\hline$E_{\text {miss }}<0.19 \mathrm{GeV}$ & $1.9 \%$ & $1.8 \%$ & $1.9 \%$ \\
\hline Total uncertainty & $3.4 \%$ & $3.6 \%$ & $3.9 \%$ \\
\hline
\end{tabular}

\begin{tabular}{|c|c|c|c|}
\hline SRC Cut & $\mathrm{Al} / \mathrm{C}$ & $\mathrm{Fe} / \mathrm{C}$ & $\mathrm{Pb} / \mathrm{C}$ \\
\hline \hline$x_{B}>1.1$ & $0.83 \%$ & $1.5 \%$ & $2.0 \%$ \\
\hline${ }^{*} \theta_{p q}<25^{\circ}$ & $2.0 \%$ & $2.5 \%$ & $2.4 \%$ \\
\hline${ }^{*} 0.62<p / q<1.1$ & & & \\
\hline$M_{\text {miss }}<1.175 \mathrm{GeV} / \mathrm{c}^{2}$ & $1.9 \%$ & $2.1 \%$ & $2.2 \%$ \\
\hline $0.4<P_{\text {miss }}<1 \mathrm{GeV} / \mathrm{c}$ & $2.2 \%$ & $1.9 \%$ & $2.6 \%$ \\
\hline Total uncertainty & $3.6 \%$ & $4.1 \%$ & $4.6 \%$ \\
\hline
\end{tabular}

Table 11: The event-selection cuts, and the change in the ratios due to variations in the cuts, for smeared protons. 
Figure 61 shows the resulting $A$-dependence of the $\left(e, e^{\prime} p\right)$ cross-section ratios for smeared (red) and un-smeared (blue) protons, for low-momentum (left) and high-momentum (right) kinematics. As can be seen, the smeared results agree well with the un-smeared protons results indicating that we corrected for bin migration (false positive and negative) correctly.

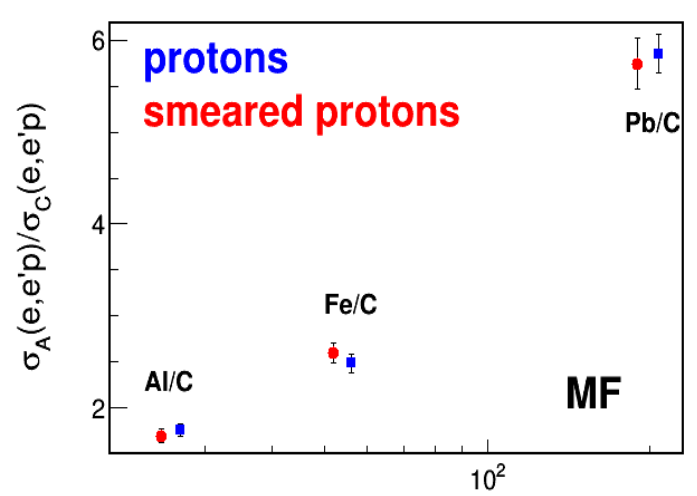

A

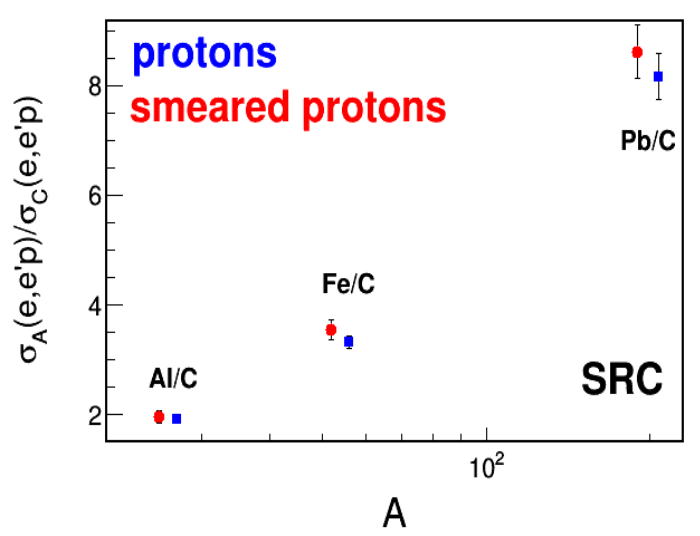

Figure 61: The $A$-dependence of the $A\left(e, e^{\prime} p\right) / C\left(e, e^{\prime} p\right)$ cross-section ratios for smeared protons (red), and unsmeared protons (blue), for mean-field (left) and high-momentum kinematics (right).

- $\frac{A\left(e, e^{\prime} n\right)}{C\left(e, e^{\prime} n\right)}$ ratios

After showing the equivalence of the smeared and un-smeared protons, we performed a "blind" analysis in order to extract the same ratios for neutrons. The corrections and contributions for the uncertainty are the same as for the proton ratios. Table 12 summarizes the sensitivity of the neutron ratios to the event selection cuts. Figure 62 shows the resulting $A$-dependence of the $\left(e, e^{\prime} n\right)$ cross-section ratios for low-momentum (left) and high-momentum (right) kinematics.

\begin{tabular}{|c|c|c|c|}
\hline MF Cut & $\mathrm{Al} / \mathrm{C}$ & $\mathrm{Fe} / \mathrm{C}$ & $\mathrm{Pb} / \mathrm{C}$ \\
\hline \hline$-0.05<y<0.25$ & $0.8 \%$ & $1.3 \%$ & $1.2 \%$ \\
\hline $0.95<\omega<1.7 \mathrm{GeV}$ & $1.4 \%$ & $1.2 \%$ & $1.7 \%$ \\
\hline$\theta_{p q}<8^{\circ}$ & $1.5 \%$ & $1.6 \%$ & $1.0 \%$ \\
\hline$P_{\text {miss }}<0.3 \mathrm{GeV} / \mathrm{c}$ & $1.2 \%$ & $1.3 \%$ & $1.5 \%$ \\
\hline$E_{\text {miss }}<0.19 \mathrm{GeV}$ & $0.8 \%$ & $0.9 \%$ & $1.4 \%$ \\
\hline Total uncertainty & $2.6 \%$ & $2.9 \%$ & $3.1 \%$ \\
\hline
\end{tabular}

\begin{tabular}{|c|c|c|c|}
\hline SRC Cut & $\mathrm{Al} / \mathrm{C}$ & $\mathrm{Fe} / \mathrm{C}$ & $\mathrm{Pb} / \mathrm{C}$ \\
\hline \hline$x_{B}>1.1$ & $1.1 \%$ & $2 \%$ & $1.9 \%$ \\
\hline${ }^{*} \theta_{n q}<25^{\circ}$ & $1.4 \%$ & $2.6 \%$ & $2.5 \%$ \\
\hline${ }^{*} 0.62<p / q<1.1$ & & & \\
\hline$M_{\text {miss }}<1.175 \mathrm{GeV} / \mathrm{c}^{2}$ & $2.4 \%$ & $3.0 \%$ & $2.0 \%$ \\
\hline $0.4<P_{\text {miss }}<1 \mathrm{GeV} / \mathrm{c}$ & $2.3 \%$ & $1.6 \%$ & $2.3 \%$ \\
\hline Total uncertainty & $3.8 \%$ & $4.7 \%$ & $4.4 \%$ \\
\hline
\end{tabular}

Table 12: Same as Table 11, for the neutrons ratios. 

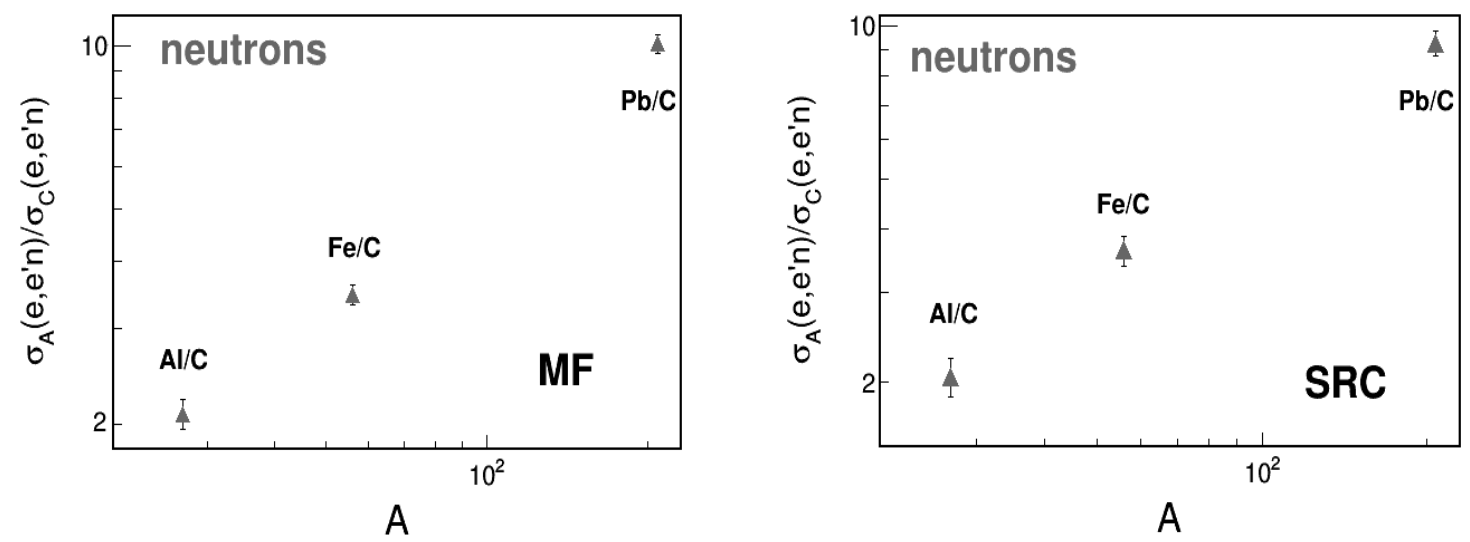

Figure 62: Same as Fig. 61 for the neutrons ratios.

\subsection{Extracting the $\frac{A\left(e, e^{\prime} N\right)_{h i g h} / A\left(e, e^{\prime} N\right)_{l o w}}{C\left(e, e^{\prime} N\right)_{h i g h} / C\left(e, e^{\prime} N\right)_{l o w}}$ double ratios}

Using the extracted ratios from the previous section, we derived the double ratios of high-momentum to low-momentum nucleons in nuclei relative to carbon, $\left[A\left(e, e^{\prime} N\right)_{\text {high }} / A\left(e, e^{\prime} N\right)_{\text {low }}\right] /\left[C\left(e, e^{\prime} N\right)_{\text {high }} / C\left(e, e^{\prime} N\right)_{\text {low }}\right]$. The double ratio is an estimator for the increased fraction of SRC nucleons in an asymmetric nuclei compared to carbon. We used carbon as a reference because it is a well studied, medium-mass symmetric nucleus and has a similar average density to the other nuclei measured here. In addition, these cross-section ratios relative to carbon are very robust as normalization, transparency, and proton (neutron) detection efficiency corrections cancel in the high-momentum to low-momentum ratio for the same nuclei. See Table 13 for the different contributions to the double-ratio uncertainties. Figure 63 shows the resulting high-momentum fractions for protons and neutrons for the different nuclei. We found that the fraction of high-initial-momentum protons increaces by about $50 \%$ from carbon to lead. Moreover, the corresponding fraction of high-initial-momentum neutrons seems to decrease by about $10 \% \pm 5 \%(1 \sigma)$. Nucleon rescattering, if substantial, should increase in larger nuclei and should affect protons and neutrons equally. Because, unlike the proton ratio, the neutron ratio decreases slightly with mass number, this also rules out sizeable nucleon rescattering effects. Figure 63 also shows the results of the simple phenomenological $n p$-dominance model presented in Chapter 1, at which we used a mean-field momentum distribution at low-momentum, and a scaled deutron-like high-momentum tail. This model agrees with our data and also predicts momentum-sharing inversion, i.e., on average protons move faster than neutrons in neutron-rich nuclei. 


\begin{tabular}{|c|c|c|c|c|c|}
\hline protons & measured ratio & Event selection & False positive and negative & Statistics & Prediction \\
\hline \hline $\mathrm{Al} / \mathrm{C}$ & $1.15 \pm 0.09$ & \pm 0.06 & \pm 0.01 & \pm 0.06 & $1.06-1.17$ \\
\hline $\mathrm{Fe} / \mathrm{C}$ & $1.36 \pm 0.08$ & \pm 0.07 & \pm 0.01 & \pm 0.03 & $1.20-1.30$ \\
\hline $\mathrm{Pb} / \mathrm{C}$ & $1.50 \pm 0.10$ & \pm 0.09 & \pm 0.02 & \pm 0.04 & $1.44-1.60$ \\
\hline \hline neutrons & measured ratio & Event selection & False positive and negative & Statistics & Prediction \\
\hline \hline $\mathrm{Al} / \mathrm{C}$ & $0.99 \pm 0.10$ & \pm 0.05 & \pm 0.01 & \pm 0.09 & $0.97-1.07$ \\
\hline $\mathrm{Fe} / \mathrm{C}$ & $1.05 \pm 0.08$ & \pm 0.06 & \pm 0.01 & \pm 0.05 & $0.92-1.03$ \\
\hline $\mathrm{Pb} / \mathrm{C}$ & $0.92 \pm 0.06$ & \pm 0.05 & \pm 0.01 & \pm 0.03 & $0.71-0.83$ \\
\hline
\end{tabular}

Table 13: The measured double-ratios and their total uncertainties shown in Fig. 63 ( $2^{\text {nd }}$ column), the different contributions for the uncertainties $\left(3^{r d}-5^{t h}\right.$ columns), and the predicted ratios based on the phenomenological $n p$ dominance model ( $6^{\text {th }}$ column). The range in the predictions is due to the use of different models for the mean-field momentum distributions and different values for the transition momentum $k_{0}$ (see Sec. 1 for details).

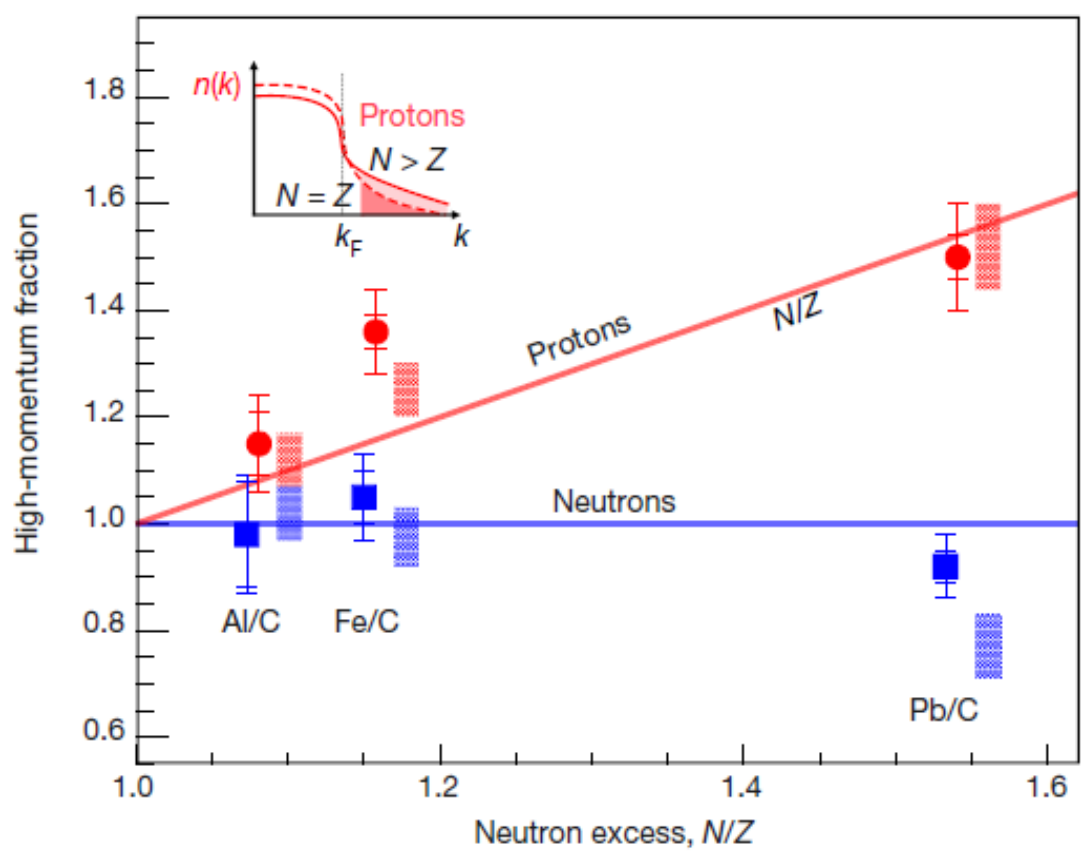

Figure 63: Relative high-momentum fractions for protons (red circles) and neutrons (blue squares). The inner error bars are statistical and the outer ones include both statistical and systematic uncertainties. Red and blue rectangles show the range of predictions of the phenomenological $n p$-dominance model for proton and neutron ratios, respectively. The red line (high-momentum fraction equal to $N / Z$ ) and the blue line (high-momentum fraction equal to 1) are drawn to guide the eye. The inset demonstrates how adding neutrons to the target nucleus (solid red curve) increases the fraction of protons in the high-momentum tail (shaded region).

\subsection{Extracting the $\frac{A\left(e, e^{\prime} n\right)}{A\left(e, e^{\prime} p\right)}$ ratios}

Using the selected events described in Sec. 3.2 we extracted the neutron-to-proton reduced crosssection ratios, for both low and high initial momenta: $\left[A\left(e, e^{\prime} n\right) / \sigma_{n}\right] /\left[A\left(e, e^{\prime} p\right) / \sigma_{p}\right]$, that is, the ratio of measured cross-sections for the scattering of electrons from nucleus $A$, scaled by the known elastic-scattering electron-neutron, $\sigma_{n}$, and electron-proton $\sigma_{p}$, cross-sections. We started with ${ }^{12} \mathrm{C}$ as a sanity check, to verify the extraction (neutron detection efficiency, detector acceptances...). Since it is a symmetric nucleus, both ratios, at low and high momenta should be equal to unity. 
As mentioned above, since we are looking at the ratio of neutrons to protons, we use the reduced cross-section which is defined as follows:

$$
\sigma_{n(p)}=\left[\left(\frac{d \sigma}{d \Omega}\right)_{e x p} /\left(\frac{d \sigma}{d \Omega}\right)_{M}\right] \frac{\epsilon(1+\tau)}{\tau}=\frac{\epsilon}{\tau} G_{E}^{2}+G_{M}^{2}
$$

where $\left(\frac{d \sigma}{d \Omega}\right)_{\text {exp }}$ is a measured cross-section, $\left(\frac{d \sigma}{d \Omega}\right)_{M}$ is the Mott cross-section, $G_{E}$ is the electric form factor (FF) of the neutron (proton), $G_{M}$ is the magnetic form factor, $\tau=\frac{Q^{2}}{4 M_{N}^{2}}$ is a kinematic factor, $M_{N}$ is the nucleon mass, $\epsilon=\left[1+2(1+\tau) \tan ^{2} \frac{\theta_{e}}{2}\right]^{-1}$, and $\theta_{e}$ is the scattering angle of the electron. The neutron and proton FF were taken from [25]. Due to the dependence of the cross-sections on $Q^{2}$ and $\theta_{e}$, we scaled the cross-section ratio event-by-event.

Even though many of the corrections cancel when considering ratios for the same nucleus, the extraction of this cross-section ratio still requires several corrections:

- Acceptance correction and detection efficiency - We consider in this work only partial phase-space events that have the same CLAS acceptance for both neutrons and protons. The neutron-detection efficiency was measured as discussed above in Sec. 3.1.5, whereas the proton-detection efficiency in this region of phase-space was estimated using the CLAS Geant-3 Monte-Carlo simulation. All these corrections were applied on an event-by-eveny basis. Below we described in more details the proton correction.

We define the proton detection efficiency within CLAS aaceptance as $\epsilon_{p}=\frac{N_{r e c}}{N_{g e n}}$, where $N_{\text {rec }}$ is the number of reconstructed events, and $N_{\text {gen }}$ is the number of generated events, in each kinematics bin. The reconstructed events are generated events that were identified in the detector as determined by the simulation. The accepted and detection efficiency corrections were obtained using the CLAS Geant-3 41 Monte-Carlo simulation process GSIM [20] $\rightarrow$ GPP (GSIM Post Processing package) $20 \rightarrow$ RECSIS 42, in the following steps:

1. Event generator:

- We chose 10,000 electrons from our data sample, ones that we know that were found in the detector and passed our cuts. For each one of them we take the momentum vector $\vec{p}_{e}$.

- We randomized (uniformly distributed) the proton momentum and scattering angle (in our kinematic regime).

- For each event from the last step, we randomized 100 times the phi angle, assuming a uniform distribution $\left(-180^{\circ} \leq \phi_{p} \leq 180^{\circ}\right)$. This results in $1,000,000$ generated $\left(e, e^{\prime} p\right)$ events.

2. We ran the $\left(e, e^{\prime} p\right)$ sample through the CLAS Geant-3 Monte-Carlo simulation: The GSIM program is a software model of the CLAS spectrometer, and allows to model its response to the passage of particles through. It includes processes such as energy loss and radiation of secondary particles during the transport through different parts of CLAS. The input data to GSIM is a set of four-momenta of particles created by the event generator. In order to eliminate signals from known 
dead channels and to smear the particles according to CLAS resolution, we run the sample through the GSIM Post, GPP. Eventually, we run the sample through RECSIS, the CLAS reconstruction system.

3. We corrected the data event-by-event by scaling each event by the ratio of reconstructed/generated events from the simulation. The correction was done to each momentum and scattering angle bin of the proton, and for each sector.

Figure 64 shows the simulated corrections as a function of the proton scattering angle and its momentum, for each sector.

Note that by using this procedure, we are taking into account the electron acceptance in the detector. However, the electron acceptance is the same for $\left(e, e^{\prime} p\right)$ and $\left(e, e^{\prime} n\right)$ events. Therefore, in order to cancel out electron acceptance effects, we take the number of generated events to be the number of $\left(e, e^{\prime}\right)$ events that were reconstructed.

As can be seen in Fig. 64, there is a sharp decrease in the efficiency in some sectors, at certain angular regions. This is due to dead detector regions in some of CLAS sectors. These regions were removed from the analysis of all targets, both for protons and neutrons. The dead regions did not change during the experiment.

- Transparency difference for neutrons and protons - At high momentum $(>1 \mathrm{GeV} / \mathrm{c})$ the proton-proton and neutron-neutron scattering cross-sections are similar. Therefore, the neutron and proton transparencies are practically the same and cancel in the $A\left(e, e^{\prime} p\right) / A\left(e, e^{\prime} n\right)$ ratio. A possible exception is in the case of highly asymmetric nuclei like ${ }^{208} \mathrm{~Pb}$, due to the large excess of neutrons. Such a difference between the proton and neutron transparencies was estimated using relativistic Glauber calculations and was found to be within $1 \%$. Therefore, we did not correct the data for that, but we did include a $1 \%$ uncertainty to the ratios of ${ }^{208} \mathrm{~Pb}\left(e, e^{\prime} p\right) /{ }^{208} \mathrm{~Pb}\left(e, e^{\prime} n\right) \mathrm{QE}$ events. See more details on the transparencies of neutron and proton in Sec. 4.5 of this work.

The contributions to the uncertainty include:

- Statistics

- Sensitivity to the event selection cuts - Estimated in the same way as in Sec. 4.1. Table 14 lists the event selection cuts used in each kinematics and the changes in the relative event yield. 


\begin{tabular}{|c|c|c|c|c|}
\hline MF Cut & $\mathrm{C}$ & $\mathrm{Al}$ & $\mathrm{Fe}$ & $\mathrm{Pb}$ \\
\hline \hline$-0.05<y<0.25$ & $0.84 \%$ & $0.83 \%$ & $0.58 \%$ & $0.81 \%$ \\
\hline $0.95<\omega<1.7 \mathrm{GeV}$ & $2.1 \%$ & $2.0 \%$ & $1.9 \%$ & $1.8 \%$ \\
\hline$\theta_{p q}<8^{\circ}$ & $2.0 \%$ & $1.8 \%$ & $1.6 \%$ & $1.4 \%$ \\
\hline$P_{\text {miss }}<0.3 \mathrm{GeV} / \mathrm{c}$ & $0.82 \%$ & $0.49 \%$ & $0.56 \%$ & $0.78 \%$ \\
\hline$E_{\text {miss }}<0.19 \mathrm{GeV}$ & $1.9 \%$ & $2.2 \%$ & $2.1 \%$ & $2.1 \%$ \\
\hline Total uncertainty & $3.7 \%$ & $3.6 \%$ & $3.4 \%$ & $3.3 \%$ \\
\hline
\end{tabular}

\begin{tabular}{|c|c|c|c|c|}
\hline SRC Cut & $\mathrm{C}$ & $\mathrm{Al}$ & $\mathrm{Fe}$ & $\mathrm{Pb}$ \\
\hline \hline$x_{B}>1.1$ & $1.0 \%$ & $0.9 \%$ & $1.3 \%$ & $1.4 \%$ \\
\hline${ }^{*} \theta_{n q}<25^{\circ}$ & $1.9 \%$ & $1.8 \%$ & $2.1 \%$ & $2.3 \%$ \\
\hline${ }^{*} 0.62<p / q<1.1$ & & & & \\
\hline$M_{\text {miss }}<1.175 \mathrm{GeV} / \mathrm{c}^{2}$ & $2.1 \%$ & $2.0 \%$ & $2.2 \%$ & $1.8 \%$ \\
\hline $0.4<P_{\text {miss }}<1 \mathrm{GeV} / \mathrm{c}$ & $2.3 \%$ & $2.4 \%$ & $2.5 \%$ & $2.3 \%$ \\
\hline Total uncertainty & $3.8 \%$ & $3.7 \%$ & $4.1 \%$ & $4.0 \%$ \\
\hline
\end{tabular}

Table 14: The event selection cuts, and the change in the ratios due to variations in the cuts, for $A\left(e, e^{\prime} n\right) / A\left(e, e^{\prime} p\right)$ ratios.
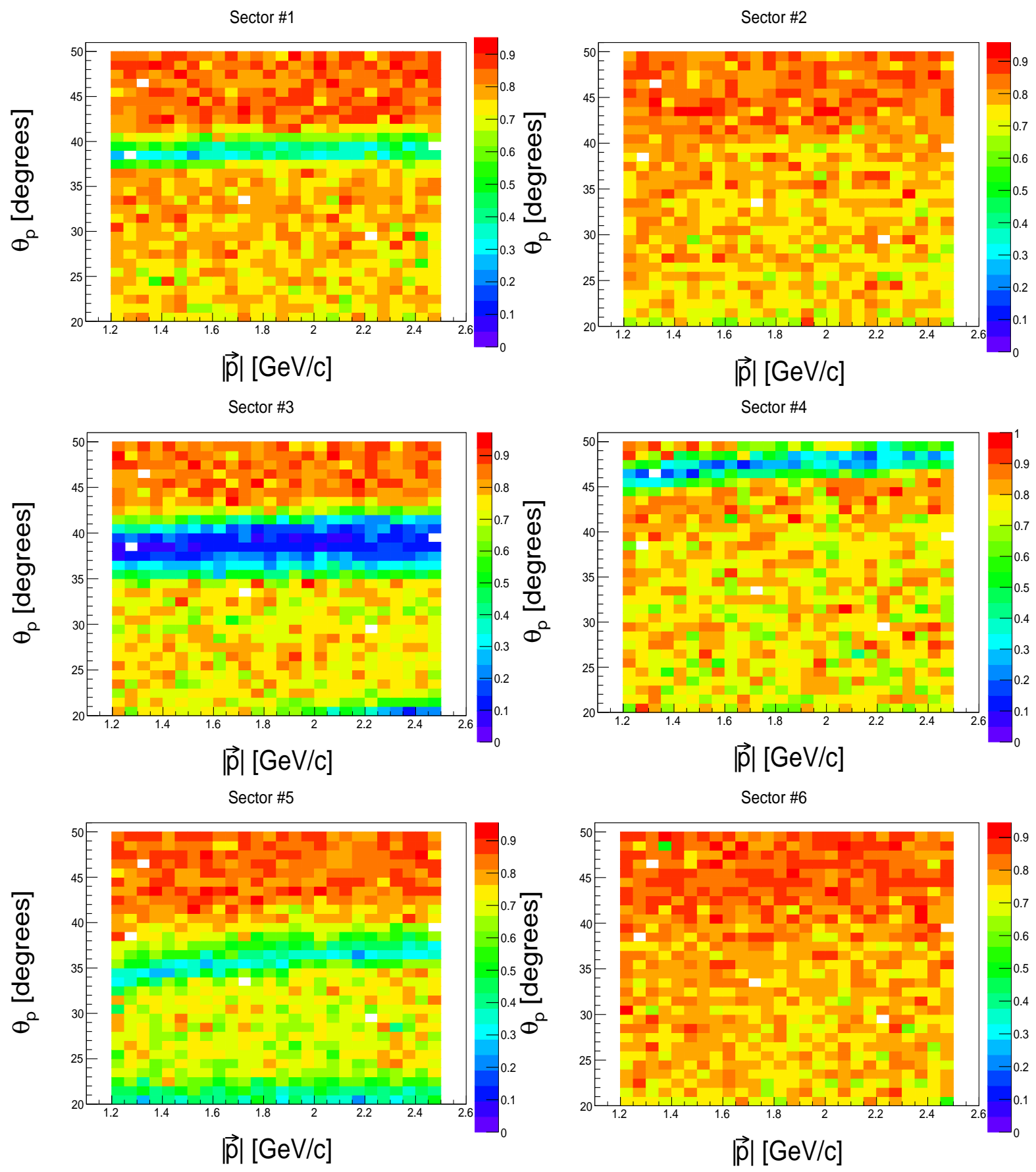

Figure 64: The simulated corrections factor as a function of the scattering angle of the proton and its momentum. 
- Acceptance correction and detection efficiency for the protons - Estimated using the CLAS Geant-3 simulation. As described above, we took protons which are uniformly distributed in their momentum. To evaluate the uncertainty, we used a different input model for the simulation, and took the difference between the resulted simulated corrections as the uncertainty.

- Neutron detection efficiency - In Sec. 3.1.5 we extracted the neutron detection efficiency for each sector as a function of the measured momentum, and fitted it with Eq. 8. The detection efficiency uncertainty was calculated based on the errors of the fitted parameters (see Tab. 5).

- Transparency - As discussed above.

Tables 15 and 16 summarize the $A\left(e, e^{\prime} n\right) / A\left(e, e^{\prime} p\right)$ M.F. and SRC ratios and their uncertainties.

\begin{tabular}{|c|c|c|c|c|c|c|}
\hline Nuclei & $A\left(e, e^{\prime} n\right) / A\left(e, e^{\prime} p\right)$ & Statistics & Neutron efficiency & Proton efficiency \& acceptance & Event selection & Transparency \\
\hline \hline${ }^{12} \mathrm{C}$ & $1.01 \pm 0.05$ & \pm 0.01 & \pm 0.03 & \pm 0.01 & \pm 0.04 & - \\
\hline${ }^{27} \mathrm{Al}$ & $1.10 \pm 0.06$ & \pm 0.02 & \pm 0.04 & \pm 0.01 & \pm 0.04 & \\
\hline${ }^{56} \mathrm{Fe}$ & $1.12 \pm 0.05$ & \pm 0.01 & \pm 0.03 & \pm 0.01 & \pm 0.04 & - \\
\hline${ }^{208} \mathrm{~Pb}$ & $1.67 \pm 0.10$ & \pm 0.03 & \pm 0.07 & \pm 0.03 & \pm 0.05 & \pm 0.02 \\
\hline
\end{tabular}

Table 15: $\frac{A\left(e, e^{\prime} n\right)}{A\left(e, e^{\prime} p\right)}$ M.F. ratios and the different contributions to the uncertainty.

\begin{tabular}{|c|c|c|c|c|c|c|}
\hline Nuclei & $A\left(e, e^{\prime} n\right) / A\left(e, e^{\prime} p\right)$ & Statistics & Neutron efficiency & Proton efficiency \& acceptance & Event selection & Transparency \\
\hline \hline${ }^{12} \mathrm{C}$ & $1.10 \pm 0.07$ & \pm 0.03 & \pm 0.04 & \pm 0.02 & \pm 0.04 & - \\
\hline${ }^{27} \mathrm{Al}$ & $1.04 \pm 0.07$ & \pm 0.04 & \pm 0.03 & \pm 0.02 & \pm 0.04 & - \\
\hline${ }^{56} \mathrm{Fe}$ & $0.96 \pm 0.05$ & \pm 0.02 & \pm 0.03 & \pm 0.01 & \pm 0.04 & - \\
\hline${ }^{208} \mathrm{~Pb}$ & $1.00 \pm 0.07$ & \pm 0.04 & \pm 0.03 & \pm 0.02 & \pm 0.04 & \pm 0.01 \\
\hline
\end{tabular}

Table 16: $\frac{A\left(e, e^{\prime} n\right)}{A\left(e, e^{\prime} p\right)}$ SRC ratios and the different contributions to the uncertainty.

As can be seen in Fig. 65, for ${ }^{12} \mathrm{C}$, in both the low- and high-initial-momentum, the ratios are consistent with unity, as expected for a symmetric nucleus. For the other measured nuclei, the lowmomentum $\left(e, e^{\prime} n\right) /\left(e, e^{\prime} p\right)$ reduced cross-section ratios grow approximately as $N / Z$, as expected from the number of neutrons $(N)$ and protons $(Z)$ in the nucleus. However, the high-momentum $\left(e, e^{\prime} n\right) /\left(e, e^{\prime} p\right)$ are consistent with unity for all measured nuclei, independent of the neutron excess.

The struck nucleons could reinteract as they emerge from the nucleus, which we refer to as finalstate interaction (FSI). Such an effect would cause the number of detected outgoing nucleons to decrease and also modify the angles and momenta of the knocked-out nucleons. These effects were estimated for symmetric and asymmetric nuclei using a relativistic Glauber framework, which showed that the decrease in the measured cross-section is similar for protons and neutrons and thus has a minor impact on cross-section ratios (see [26]). 
Because rescattering changes the event kinematics, some of the events with high measured $p_{\text {miss }}$ could have originated from electron scattering from a low-initial-momentum nucleon, which then rescattered, thus increasing $p_{\text {miss }}$. If the high-initial-momentum (high- $p_{m i s s}$ ) nucleons originated from electron scattering from the more numerous low-initial-momentum nucleons, followed by nucleon rescattering, then the high-momentum $\left(e, e^{\prime} n\right) /\left(e, e^{\prime} p\right)$ ratio would show the same $N / Z$ dependence as the low-momentum ratio. Because the high-momentum $\left(e, e^{\prime} n\right) /\left(e, e^{\prime} p\right)$ ratio is independent of $A$, these nucleon-rescattering effects must be small in this measurement.

Thus, the constant $\left(e, e^{\prime} n\right) /\left(e, e^{\prime} p\right)$ high-momentum ratios indicate that there are equal numbers of high-initial-momentum protons and neutrons in asymmetric nuclei, even though these nuclei contain up to $50 \%$ more neutrons than protons. This observation is consistent with the claim that the high-initial-momentum nucleons belonging primarily to $n p$-SRC pairs, even in neutron-rich nuclei.

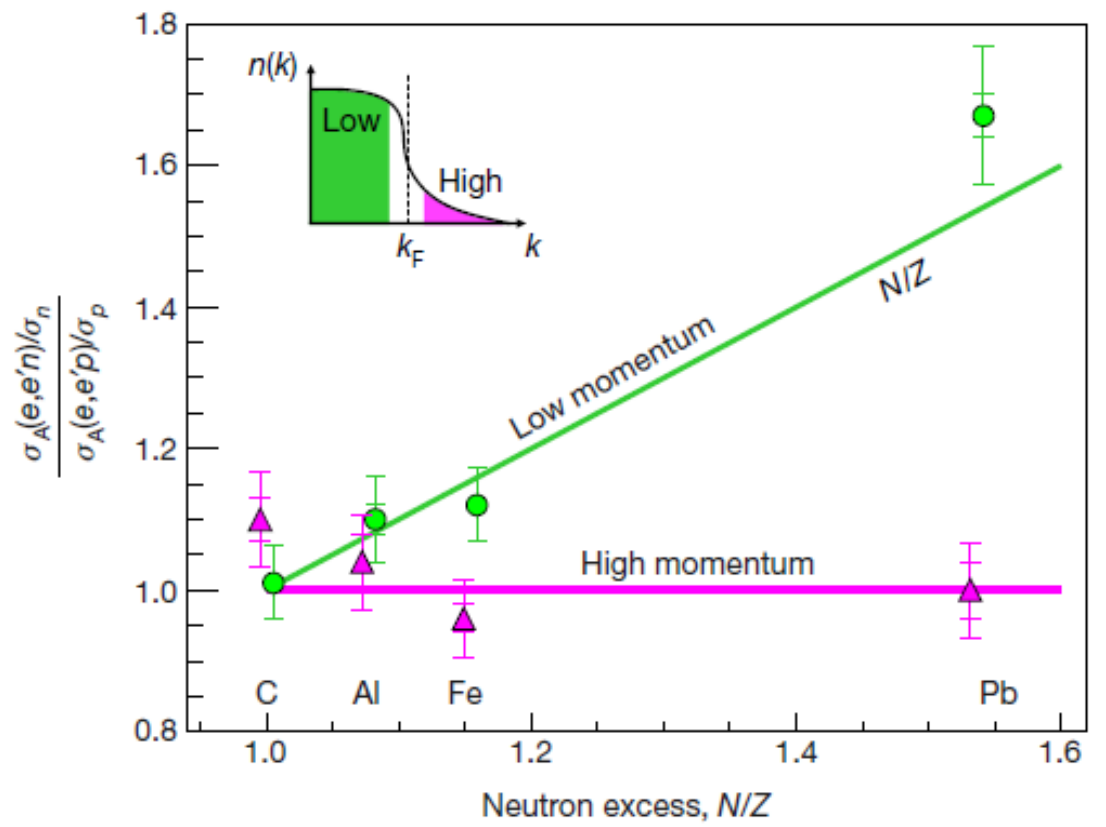

Figure 65: Relative abundances of high- and low-initial-momentum neutrons and protons. Reduced cross-section ratio, $\left[\sigma_{A}\left(e, e^{\prime} n\right) / \sigma_{n}\right] /\left[\sigma_{A}\left(e, e^{\prime} p\right) / \sigma_{p}\right]$, for low-momentum (green circles) and high-momentum (purple triangles) events. The inset illustrates a typical nuclear momentum distribution as a function of nucleon momentum, where 'low' and 'high' refer to the initial nucleon momentum. The lines show the simple $N / Z$ behaviour (green), as expected from the number of neutrons and protons in the nucleus for low-momentum nucleons, and the prediction of the $n p$-dominance (purple; $\left.\left[\sigma_{A}\left(e, e^{\prime} n\right) / \sigma_{n}\right] /\left[\sigma_{A}\left(e, e^{\prime} p\right) / \sigma_{p}\right]=1\right)$ for high-momentum nucleons. The inner error bars correspond to statistical uncertainties and the outer ones include both statistical and systematic uncertainties, both at the $1 \sigma$ or $68 \%$ confidence level.

\subsection{Triple coincidence ratios}

Using the selected $\left(e, e^{\prime} p p\right)$ and $\left(e, e^{\prime} n p\right)$ events described in Sec. 3.3 , we extracted the $A\left(e, e^{\prime} p p\right) / A\left(e, e^{\prime} n p\right)$ cross-section ratios, scaled by the elementary electron-proton and electron-neutron cross-sections, $\sigma_{p}$ and $\sigma_{n}, \sigma_{p / n}=\sigma_{p} / \sigma_{n}$ (in the same way as in Sec. 4.3), and by the number of scattered protons (1 or 2$)$. 
The resulting reduced cross-section ratio $R=\left[A\left(e, e^{\prime} p p\right) / 2 \cdot \sigma_{p}\right] / A\left(e, e^{\prime} n p\right) / \sigma_{n}$ for all measured nuclei is shown in Fig. 66. The inner error bars show the statistical uncertainties while the outer ones include systematic uncertainties added in quadrature. The latter include sensitivity of the extracted cross-section ratio to the event selection cuts detailed in Table 17, uncertainties in the neutron and proton detection efficiency and a the small difference for the leading proton and neutron transparency in lead (same as in Sec. 4.3). The ratios and the different contributions to the uncertainties are summarized in Table 18.

\begin{tabular}{|c|c|c|c|c|c|}
\hline Cut & Sensitivity range & ${ }^{12} \mathrm{C}$ & ${ }^{27} \mathrm{Al}$ & ${ }^{56} \mathrm{Fe}$ & ${ }^{208} \mathrm{~Pb}$ \\
\hline \hline$x_{B}>1.1$ & \pm 0.05 & $1.5 \%$ & $1.9 \%$ & $1.4 \%$ & $1.7 \%$ \\
\hline${ }^{*} \theta_{p q}<25^{\circ}$ & $\pm 5^{\circ}$ & $2.7 \%$ & $2.5 \%$ & $2.3 \%$ & $2.2 \%$ \\
\hline${ }^{*} 0.62<p / q<1.1$ & \pm 0.05 & & & & \\
\hline$m_{\text {miss }}<1.175 \mathrm{GeV} / \mathrm{c}^{2}$ & $\pm 0.025 \mathrm{GeV} / \mathrm{c}^{2}$ & $2.4 \%$ & $2.3 \%$ & $3.1 \%$ & $2.0 \%$ \\
\hline $0.4<p_{\text {miss }}<1 \mathrm{GeV} / \mathrm{c}$ & $\pm 0.025 \mathrm{GeV} / \mathrm{c}$ & $2.6 \%$ & $2.8 \%$ & $2.1 \%$ & $2.1 \%$ \\
\hline$p_{\text {rec }}>0.35 \mathrm{GeV} / \mathrm{c}$ & $\pm 0.025 \mathrm{GeV} / \mathrm{c}$ & $2.4 \%$ & $2.6 \%$ & $2.3 \%$ & $2.7 \%$ \\
\hline SC Deposited Energy & cut ON/OFF & $0.2 \%$ & $3.2 \%$ & $1.0 \%$ & $2.3 \%$ \\
\hline Total uncertainty & & $5.3 \%$ & $6.3 \%$ & $5.2 \%$ & $5.4 \%$ \\
\hline
\end{tabular}

Table 17: The $\left(e, e^{\prime} N p\right)$ event selection cuts. Also shown is the sensitivity of the $p p / n p$ ratios to variations of the cuts within the ranges shown in the second column. *Both leading nucleon cuts were varied simultaneously.

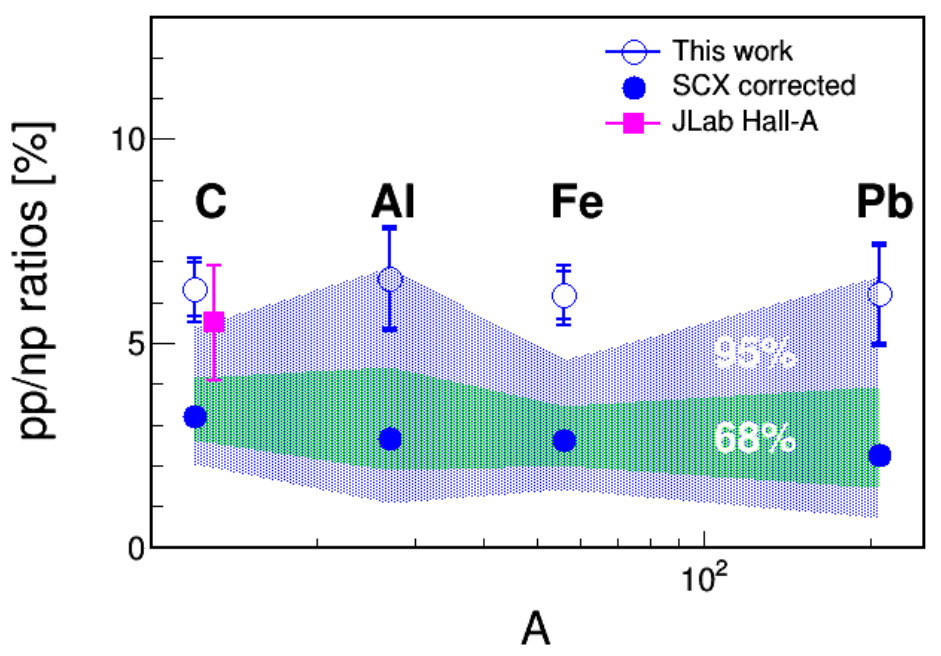

Figure 66: Extracted ratios of $p p$ - to $n p$-SRC pairs in nuclei. The open symbols show the measured reduced crosssection ratios $R=\left[A\left(e, e^{\prime} p p\right) / 2 \cdot \sigma_{p}\right] /\left[A\left(e, e^{\prime} n p\right) / \sigma_{n}\right]$. The filled symbols show the extracted ratios of $p p$ - to $n p$-SRC pairs obtained from the measured cross-section ratios after SCX corrections using Eq. 15. The magenta square shows the data of [27, which were also corrected for SCX. The shaded regions mark the $68 \%$ and $95 \%$ confidence limits on the extraction due to uncertainties in the measured cross-section ratios and SCX correction factors (see Appendix A for details). 


\begin{tabular}{|c|c|c|c|c|c|c|}
\hline$A$ & $\frac{A\left(e, e^{\prime} p p\right) / 2 \cdot \sigma_{p}}{A\left(e, e^{\prime} n p\right) / \sigma_{n}}[\%]$ & Statistics & Neutron efficiency & Proton efficiency \% acceptance & Event selection & Transparency \\
\hline \hline${ }^{12} \mathrm{C}$ & $6.31 \pm 0.79$ & \pm 0.67 & \pm 0.24 & \pm 0.10 & \pm 0.33 & - \\
\hline${ }^{27} \mathrm{Al}$ & $6.57 \pm 1.29$ & \pm 1.21 & \pm 0.18 & \pm 0.10 & \pm 0.41 & - \\
\hline${ }^{56} \mathrm{Fe}$ & $6.17 \pm 0.72$ & \pm 0.60 & \pm 0.20 & \pm 0.10 & \pm 0.32 & - \\
\hline${ }^{208} \mathrm{~Pb}$ & $6.19 \pm 1.26$ & \pm 1.20 & \pm 0.19 & \pm 0.10 & \pm 0.33 & \pm 0.06 \\
\hline
\end{tabular}

Table 18: The $\frac{A\left(e, e^{\prime} p p\right) / 2 \cdot \sigma_{p}}{A\left(e, e^{\prime} n p\right) / \sigma_{n}}$ M.F. ratios and the different contributions to the uncertainty.

As can be seen, the extracted reduced cross-section ratio $R$ is largely $A$-independent and equals $6 \%$. This is consistent with $n p$-SRC pairs being 20 times more abundant than $p p$-SRC pairs. However, the complete extraction of the relative abundance of $p p$ - to $n p$-SRC pairs from the measured exclusive two-nucleon knockout cross-section ratios require correcting for reaction mechanism effects. According to calculations 43, 44, 45, reaction mechanisms other than the hard breakup of SRC pairs are suppressed under the conditions of our measurement. The residual effects of nonQE reaction mechanisms are significantly reduced in the cross-section ratios as compared to the absolute cross-sections. At the relevant high- $Q^{2}$ range, the cross-sections approximately factorize and calculations of final state interactions (FSI) and single-charge exchange (SCX) of the outgoing nucleons are done using an Eikonal approximation in a Glauber framework, which was shown to have good agreement with experimental data. These calculations show that FSI that do not lead to a reduction of flux are largely confined to within the nucleons of the pair. Such rescattering does not impact the isospin structure of SRC pairs, which is the main goal of the current chapter 43, 45, 28, 46. However, we need to include calculated contributions for nucleons that exit the nucleus and do SCX interactions (e.g., $(n, p)$ and $(p, n)$ reactions) that change neutrons to protons and vice versa.

At the measured outgoing nucleon momenta, the $p p$ and $n n$ elastic-scattering cross-sections are similar and therefore the nucleon attenuation is similar, i.e., the probability for a $p n$ pair to exit the nucleus in an $A\left(e, e^{\prime} n p\right)$ reaction is approximately the same as that of a $p p$ pair in the $A\left(e, e,^{\prime} p p\right)$ reaction, see [28 for details. Therefore, the SCX correction is the most signicant one. Because $n p$-SRC pairs are dominant, $n p$-pair knockout, followed by an $(n, p)$ charge-exchange reaction, could comprise a large fraction of the measured $A\left(e, e^{\prime} p p\right)$ events. This will make the extracted ratio of $p p$ - to $n p$-SRC pairs smaller than the measured reduced cross-section ratio $R$, making the latter an upper limit on the $p p$ - to $n p$-SRC pairs ratio.

Calculation of SCX effects are model and kinematics dependent. In the current analysis, we used the Glauber calculations of Ref [28] that were done for the kinematics of our measurement. We applied these SCX corrections by assuming that the measured two-nucleon knockout reactions predominantly probe SRC pairs. Under this approximation, the relative abundance of $p p$ - to $n p$-SRC pairs can be expressed as (see derivation in Appendix A):

$$
\frac{\# p p-S R C}{\# n p-S R C}=\frac{1}{2} \cdot \frac{2 \cdot R \cdot P_{A}^{n p}-P_{A}^{[n] p}-P_{A}^{p[n]} / \sigma_{p / n}}{P_{A}^{p p}-2 \cdot \sigma_{p / n} \cdot R \cdot P_{A}^{[p] p}-2 \cdot R \cdot \eta_{A} \cdot P_{A}^{n[n]}}
$$

where $\eta_{A}=\frac{\# n n-S R C}{\# p p-S R C}, P_{A}^{N N}$ is the probability for scattering off an $N N$ pair without subsequent SCX, and $P_{A}^{[N] N}$ and $P_{A}^{N[N]}$ are the probabilities for scattering off an $N N$ pair and having either the leading or recoil nucleon undergo SCX, respectively. The values and uncertainties of the 
parameters used in Eq. 15 are listed in the Appendix. While the current analysis uses the SCX calculations of Ref. 28, the formalism detailed in Appendix A, along with the measured reduced cross-section ratios shown in Fig. 66, are general and other calculations for these corrections can be applied in the future.

The SCX-corrected ratios of $p p$ - to $n p$-SRC pairs are shown in Fig. 66 by the filled points. The shaded regions mark the $68 \%$ and $95 \%$ confidence limits on this extraction due to uncertainties in the measured cross-section ratios and all other correction factors that are included in Eq. 15 (see Appendix A for details). The magenta square shows previous data from [27]. As can be seen, the SCX corrections to our data reduce the extracted $p p$ - to $n p$-SRC pairs ratio by almost a factor of 2 as compared to the uncorrected cross-section ratios, but with increased uncertainties. We can therefore deduce that the relative abundance of $n p$ - to $p p$-SRC pairs in all measured nuclei to be equal to or greater than 20. Both corrected and un-corrected ratio are summarized in Table 19 .

\begin{tabular}{|c|c|c|}
\hline$A$ & $\frac{A\left(e, e^{\prime} p p\right) / 2 \cdot \sigma_{p}}{A\left(e, e^{\prime} n p\right) / \sigma_{n}}[\%]$ & $\frac{\# p p-S R C}{\# n p-S R C}[\%]$ \\
\hline \hline${ }^{12} \mathrm{C}$ & $6.31 \pm 0.79$ & $3.21_{-0.59(1.19)}^{+0.93(2.19)}$ \\
\hline${ }^{27} \mathrm{Al}$ & $6.57 \pm 1.29$ & $2.66_{-0.79(1.59)}^{+1.75(4.21)}$ \\
\hline${ }^{56} \mathrm{Fe}$ & $6.17 \pm 0.72$ & $2.62_{-0.65(1.96)}^{+0.85(1.22)}$ \\
\hline${ }^{208} \mathrm{~Pb}$ & $6.19 \pm 1.26$ & $2.27_{-0.83(1.57)}^{+1.67(4.35)}$ \\
\hline
\end{tabular}

Table 19: Measured reduced cross-section $R$ ratios and extracted \#pp-SRC/\#np-SRC pairs ratio. The reported uncertainties in the $p p / n p$ are the $68 \%$ (95\%) confidence limits. See text for details.

\subsection{Nuclear Transparency ratios}

The nuclear transparency factor, $T(A)$, describes the probability of the outgoing nucleon to emerge from the nucleus, quantifying the multiple scattering of the knockout nucleon with the surrounding nucleons. $T(A)$ is a key ingredient in many calculations of nuclear reactions. Nuclear trancparency, calculated using the Glauber approximation, is widely used in analyses of reactions measured in high-energy, heavy-ion, and hadronic physics experiments. Therefore, the experimental extraction of transparency factors for single nucleon knockout reactions at different kinematics and for different nuclei serves as an important baseline for obtaining information on the structure and dynamics of individual nucleons bound in nuclei and as a detailed benchmark for the validity of the widely used Glauber calculations.

In this chapter we extracted the transparency ratios, $T(A) / T(C)$, for proton and neutron knockout, from $A\left(e, e^{\prime} p\right)$ and $A\left(e, e^{\prime} n\right)$ reactions. The carbon nucleus was chosen as a reference bacause it is a well-studied symmetric nucleus. The transparency ratios were extracted for the two kinematics described in this work, MF (low-momentum) and SRC (high-momentum), see Sec. 3.2.1 and 3.2.2.

The nuclear transparency is formally defined as the ratio of the experimentally extracted nucleon knock-out cross-section to the PWIA cross-section,

$$
T_{N}(A)=\frac{\sigma_{e x p} A\left(e, e^{\prime} N\right)}{\sigma_{P W I A} A\left(e, e^{\prime} N\right)}
$$


In the commonly used factorized approximation for large- $Q^{2}$ reactions, $\sigma_{P W I A}$ is given by (see 30]):

$$
\sigma_{P W I A} A\left(e, e^{\prime} N\right)=\frac{K}{\# N_{t a r}} \cdot \sigma_{e N} \cdot \oint S_{A}\left(E, p_{i}\right) d E d^{3} p_{i}
$$

in which $\# N_{\text {tar }}$ is the number of relevant nucleons in the target nucleus (i.e., number of protons for $A\left(e, e^{\prime} p\right)$ and neutrons for $\left.A\left(e, e^{\prime} n\right)\right), K=\left|\vec{p}_{N}\right| \cdot E_{N}$ is a kinematical factor, $\sigma_{e N}$ is the offshell electron-nucleon elementary cross-section, $S_{A}\left(E, p_{i}\right)$ is the nuclear spectral function, which is the probability for finding a nucleon in the nucleus with momentum $p_{i}$ and separation energy $E$. $S_{A}\left(E, p_{i}\right)$ is normalized such that ${ }_{0} \int^{\infty} S_{A}\left(E, p_{i}\right) d E d^{3} p_{i}=N_{t a r}$. The spectral function in Eq. 17 is integrated over the experimental acceptance.

If the nucleus with $A$ nucleons and ${ }^{12} \mathrm{C}$, are measured in the same kinematics, then their transparency ratio is given by:

$$
\frac{T_{N}(A)}{T_{N}(C)}=\frac{\sigma_{e x p} A\left(e, e^{\prime} N\right)}{\sigma_{e x p} C\left(e, e^{\prime} N\right)} \cdot \frac{\oint S_{C}\left(E, p_{i}\right) d E d^{3} p_{i}}{\oint S_{A}\left(E, p_{i}\right) d E d^{3} p_{i}}
$$

in which the spectral functions for $A$ and ${ }^{12} \mathrm{C}$ are integrated over the same kinematical regions.

For the MF kinematics, Eq. 18 can be expressed as:

$$
\frac{T_{N}^{M F}(A)}{T_{N}^{M F}(C)}=\frac{\sigma_{\exp } A\left(e, e^{\prime} N\right)}{\sigma_{\exp } C\left(e, e^{\prime} N\right)} \cdot \frac{0 \int^{k_{0}} n_{C}\left(p_{i}\right) d p_{i}}{0 \int^{k_{0}} n_{A}\left(p_{i}\right) d p_{i}},
$$

where $\sigma_{\text {exp }} A\left(e, e^{\prime} N\right) / \sigma_{\text {exp }} C\left(e, e^{\prime} N\right)$ is the measured nucleon knockout cross-section ratio described in Sec. 4.1 and the second term is the ratio of integrals over the mean-field part of the nuclear momentum density, which, due to the large missing-energy cut, replaces the integrals over the meanfueld spectral functions. The nuclear momentum density is defined as $n_{A}\left(p_{i}\right)=0 \int^{\infty} S_{A}\left(E, p_{i}\right) d E$ and was calculated following [31. The integral calculations in Eq. 19 were done using three different models for the mean-field momentum distribution, and two different values for $k_{0}$ (see Sec. 1.2). We assigned the half difference between the two extreme values obtained by considering the different values of $k_{0}$ and the different models as the corresponding systematic uncertainty. The values of the latter are $4.9 \%(3.8 \%), 4.2 \%(5.7 \%)$, and $4.3 \%(4.5 \%)$ for protons (neutrons) and $\mathrm{Al} / \mathrm{C}, \mathrm{Fe} / \mathrm{C}$, and $\mathrm{Pb} / \mathrm{C}$ ratios, respectively. The results of this calculation are consistent with those previously otained by Hartree-Fock-Slater wave functions 31.

For the SRC kinematics the transparency ratios were extracted following 24 as:

$$
\frac{T_{N}^{S R C}(A)}{T_{N}^{S R C}(C)}=\frac{1}{a_{2}(A / C)} \cdot \frac{\sigma_{\exp } A\left(e, e^{\prime} N\right) / A}{\sigma_{\exp } C\left(e, e^{\prime} N\right) / 12}
$$

where $a_{2}(A / C)$ is the relative number of $2 N$-SRC pairs per nucleon in nuclei $A$ and ${ }^{12} \mathrm{C}$. These ratios and their uncertainties were adapted from [32] and are based on a compilation of world data for the $\left(e, e^{\prime}\right)$ cross-section ratio at large $Q^{2}$ and $x_{B}>1$ with different theoretical corrections. 
The contributions for the systematic uncertainty of the transparency ratios include the uncertainty of the sensitivity to the event selection cuts, a $2 \%$ uncertainty of the integrated charge, and an uncertainty due to the false positive and negative correction (see Sec. 4.1 for details). For the SRC the uncertainties also include the uncertainty of $a_{2}(A / c)(5 \%)$, and a $5 \%$ uncertainty due to the $n p$-dominance assumption for the ${ }^{208} \mathrm{~Pb} /{ }^{12} \mathrm{C}$ case (see Ref. 24). For the MF, the uncertainties also include the uncertainty from the MF integrals discussed above.

Figure 67 shows the extracted transparency ratios for the various measured nuclei and kinematics. As can be seen, the extracted transparency ratios are independent of nucleon momentum between 1.4 and $2.4 \mathrm{GeV} / c$ for both proton and neutron, and for each of the three nuclei. Also shown are previous measurements for protons 16, 18, 33, 34, which are consistent with the new results. The proton knockout data are also compared to deveral Glauber calculations [31, 35, 28, 36, that show an overall good agreement with the data.

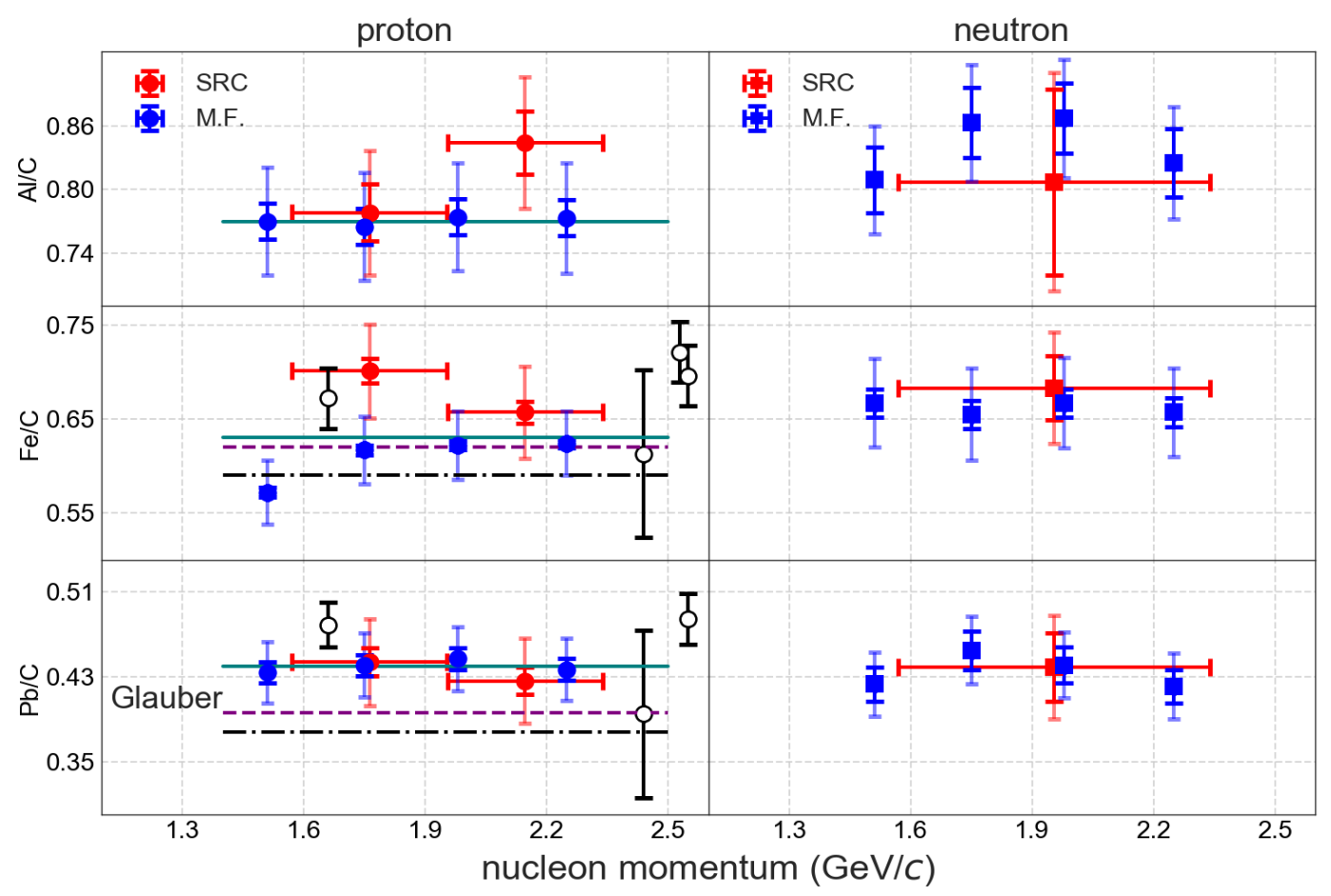

Figure 67: The extracted transparency ratios for MF and SRC kinematics, both for protons and neutrons, as a function of the nucleon momentum. Inner error bars are statistical and outer error bars include statistical and systematics uncertainties, the latter are common for the different data-points of a given measurement. The black open circles show the world data for the transparency ratios for MF proton knockout from Ref. 16 [18 33 34. Glauber calculations are shown as dotted [31, dashed [35], and solid [28, 36] lines. The nucleon momentum range for the SRC data points is denoted by the horizontal line round each point, while that of the MF data points is the same for all points and is not shown for clarity.

The proton and neutron transparency ratios, averaged over nucleon momentum, for each kinematics are listed in Table 20. 


\begin{tabular}{|c|c|c|}
\hline $\mathrm{MF}$ & $\left(e, e^{\prime} p\right)$ & $\left(e, e^{\prime} n\right)$ \\
\hline \hline $\mathrm{Al} / \mathrm{C}$ & $0.771 \pm 0.017 \pm 0.051$ & $0.853 \pm 0.033 \pm 0.054$ \\
\hline $\mathrm{Fe} / \mathrm{C}$ & $0.621 \pm 0.005 \pm 0.036$ & $0.660 \pm 0.015 \pm 0.047$ \\
\hline $\mathrm{Pb} / \mathrm{C}$ & $0.442 \pm 0.010 \pm 0.029$ & $0.439 \pm 0.017 \pm 0.031$ \\
\hline
\end{tabular}

\begin{tabular}{|c|c|c|}
\hline $\mathrm{SRC}$ & $\left(e, e^{\prime} p\right)$ & $\left(e, e^{\prime} n\right)$ \\
\hline \hline $\mathrm{Al} / \mathrm{C}$ & $0.811 \pm 0.028 \pm 0.060$ & $0.807 \pm 0.088 \pm 0.103$ \\
\hline $\mathrm{Fe} / \mathrm{C}$ & $0.679 \pm 0.013 \pm 0.048$ & $0.683 \pm 0.034 \pm 0.059$ \\
\hline $\mathrm{Pb} / \mathrm{C}$ & $0.435 \pm 0.013 \pm 0.040$ & $0.439 \pm 0.032 \pm 0.049$ \\
\hline
\end{tabular}

Table 20: Left - The transparency ratios and their uncertainties for the MF kinematics. For each nucleus, the left and the right values are for proton and neutron knockout, respectively. The first uncertainty is statistical and the second is the total. Right - Same as left, for the SRC kinematics.

Figure 68 shows the extracted transparency ratios, averaged over the momentum range shown in Fig. 67, as a function of the nuclear mass number. The momentum ranges used are 1.64-2.34 $\mathrm{GeV} / c$ (3 bins) for $\mathrm{MF}$, and 1.57-2.34 GeV/c for SRC. The transparency ratios are independent of nucleon momentum in these ranges. For example, averaging the MF transparency ratios over $1.40-2.34 \mathrm{GeV} / c$ (all 4 bins) yields a value that is within $1 \%$ (much smaller than the smallest total uncertainty) of the average over $1.64-2.34 \mathrm{GeV} / c$ (last 3 bins). Since all four transparency ratios for a given nucleus are consistent with each other within their experimental uncertainties, we take their weighted average for each nucleus.

The Glauber calculation indicates the distribution of the hard process in the nucleus, that is manifested in the nuclear mass dependence of the extracted transparencies. Following [16, 18, 33. 34, we fitted the weighted average transparencies to a power law in the form of $A^{\alpha}$ to obtain a value of $\alpha=-0.289 \pm 0.007$. This extraction is consistent with the Glauber result range of $\alpha=-0.288$ to 0.337 31, 35, 28, 36, which indicates a nuclear surface dominance of the measured reactions.

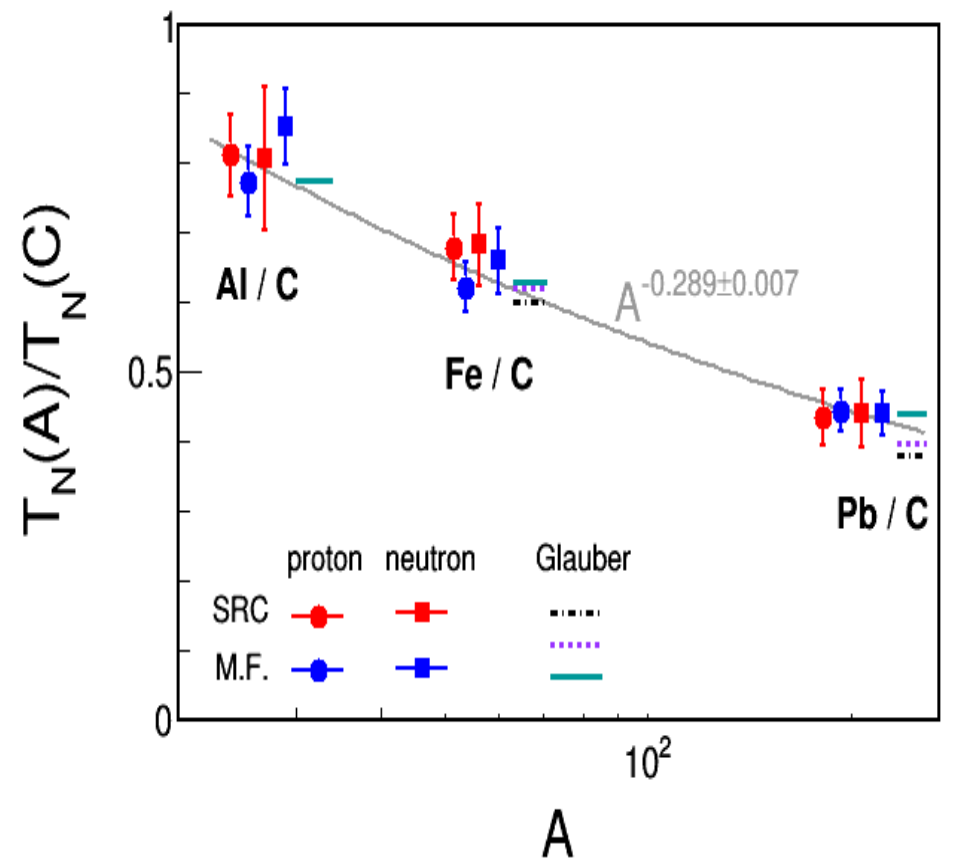

Figure 68: The extracted transparency ratios for MF and SRC kinematics, both for protons and neutrons, together with a power law fit to a weighted average (grey line), as described in the text. Also shown are results based on Glauber Calculations: 31 dotted line, 35] dashed line, and 28, 36 solid line. 


\section{$5 \quad$ Summary and Conclusion}

The analysis presented in this thesis was carried by the author (M. Duer) under the direct supervision of Prof. Eli Piasetzky (Tel-Aviv University), in close collaboration with Profs. Or Hen (MIT) and Larry Weinstein (ODU). A full analysis note including all the details, which was approved by the CLAS committee lead by Prof. Stephan Stepsnyan, is available on the web http://wwwnuclear.tau.ac.il/ eip/neutron3.pdf.

Nuclei account for nearly all the mass of the visible Universe. The atomic nucleus is one of the densest and most complex quantum-mechanical systems in nature. The properties of individual nucleons (protons and neutrons) in nuclei can be probed by scattering a high-energy particle from the nucleus and detecting this particle after it scatters, often also detecting an additional knockedout nucleon. Analysis of electron- and proton-scattering experiments suggests that some nucleons in nuclei form close-proximity neutron-proton pairs with high nucleon momentum, greater than the nuclear Fermi momentum. However, how excess neutrons in neutron-rich nuclei form such close-proximity pairs remains unclear.

In this work we measured protons and, for the first time, neutrons knocked out of medium-to-heavy nuclei by high-energy electrons. We showed that the fraction of high-momentum protons increases markedly with the neutron excess in the nucleus, whereas the fraction of high-momentum neutrons decreases slightly. This effect is surprising because in the classical nuclear shell model, protons and neutrons obey Fermi statistics, have little correlation and mostly fill independent energy shells.

The results mentioned were obtained by measuring the double ratio of high-momentum (SRC) to low-momentum (MF) nucleons in nuclei relative to carbon $\left[A\left(e, e^{\prime} N\right)_{\text {high }} / A\left(e, e^{\prime} N\right)_{\text {low }}\right] /\left[{ }^{12} C\left(e, e^{\prime} N\right)_{\text {high }} /{ }^{12} C\left(e, e^{\prime} N\right)_{\text {low }}\right]$, where $A$ stands for ${ }^{27} \mathrm{Al},{ }^{56} \mathrm{Fe}$, or ${ }^{208} \mathrm{~Pb}$. The double ratio in the chosen kinematics for this measurement is an estimator for the fraction of SRC nucleons in an asymmetric nucleus compared to carbon. We used carbon as a reference because it is a well studied, medium-mass symmetric nucleus and has similar average density to the other nuclei measured in this work. In addition, forming cross-section ratios relative to carbon significantly reduces the effects of detector acceptance and efficiency corrections. We found that the fraction of high-initial momentum protons increases by about $50 \%$ from carbon to lead. Moreover, the corresponding fraction of high-initial momentum neutrons seems to decrease by about $10 \% \pm 5 \%(1 \sigma)$. See Fig 69 . 


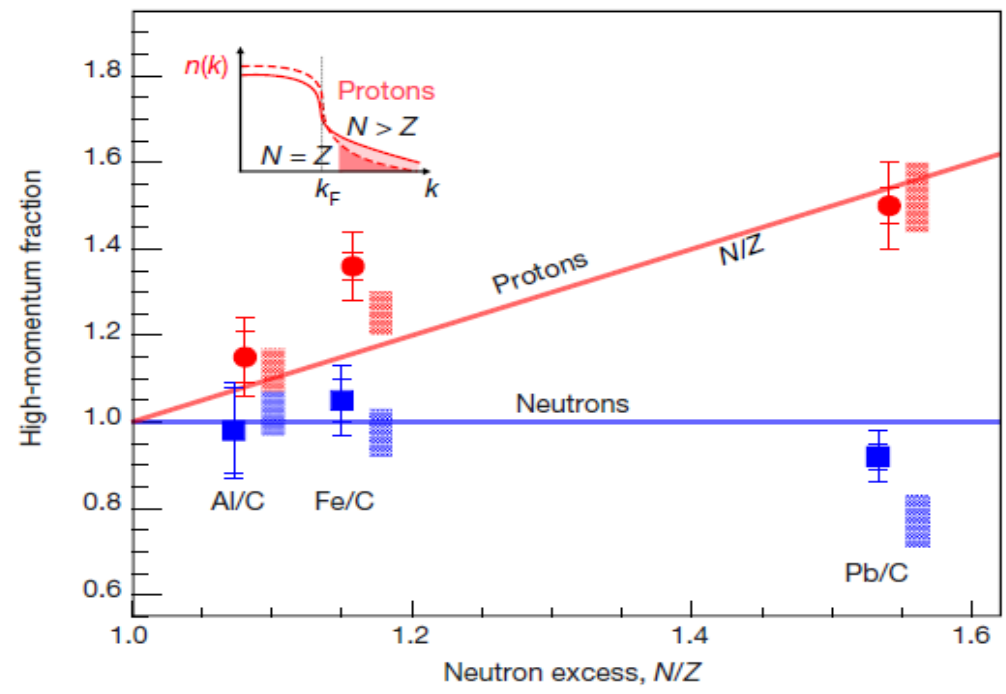

Figure 69: Relative high-momentum fractions for protons (red circles) and neutrons (blue squares). Red and blue rectangles show the range of predictions of the phenomenological $n p$-dominance model for proton and neutron ratios, respectively. The red line (high-momentum fraction equal to $N / Z$ ) and the blue line (high-momentum fraction equal to 1) are drawn to guide the eye.

In addition, we extracted the neutron-to-proton reduced cross-section ratios, for both high and low initial-nucleon momenta. For low-momentum, the $\left(e, e^{\prime} n\right) /\left(e, e^{\prime} p\right)$ reduced cross-section ratios grow approximately as $N / Z$, as expected from the number of neutrons $(N)$ and protons $(Z)$ in the nucleus. However, the high-momentum $\left(e, e^{\prime} n\right) /\left(e, e^{\prime} p\right)$ ratios are consistent with unity for all measured nuclei. See Fig 70 .

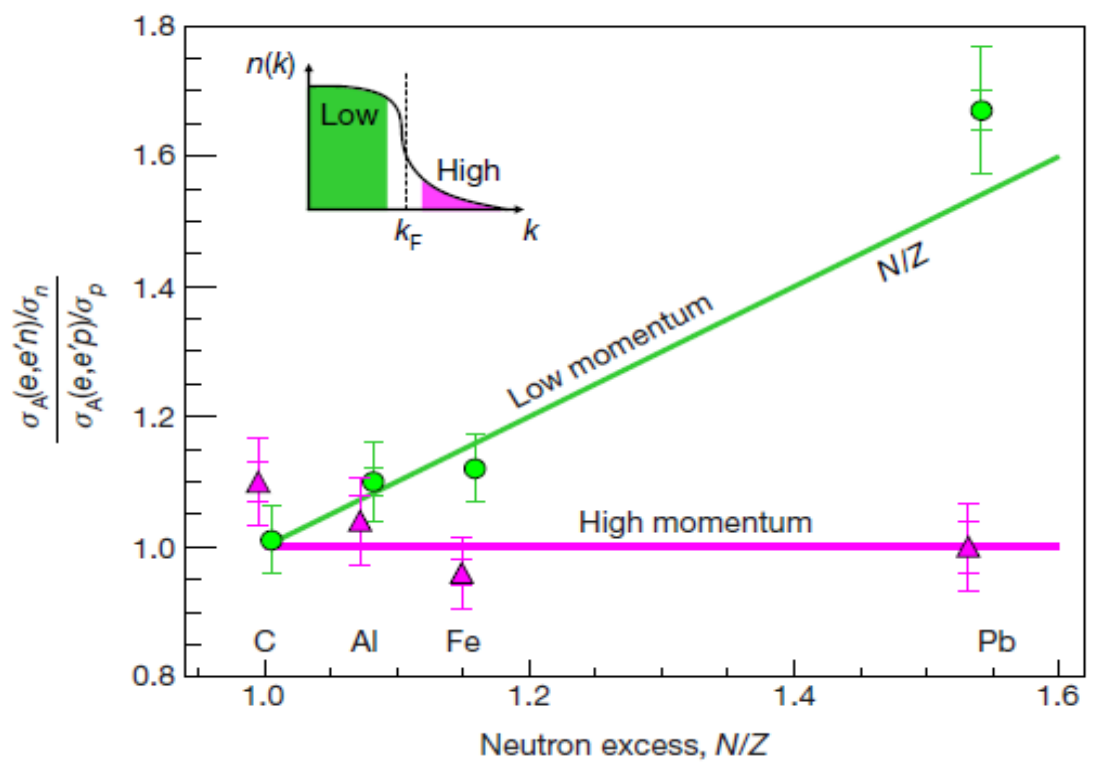

Figure 70: Relative abundances of high- and low-initial momentum neutrons and protons. Reduced crosssection ratio, $\left[\sigma_{A}\left(e, e^{\prime} n\right) / \sigma_{n}\right] /\left[\sigma_{A}\left(e, e^{\prime} p\right) / \sigma_{p}\right]$, for low-momentum (green circles) and high-momentum (purple triangles) events. The lines show the simple $N / Z$ behaviour (green), as expected from the number of neutrons and protons in the nucleus for low-momentum nucleons, and the prediction of the np-dominance (purple; $\left.\left[\sigma_{A}\left(e, e^{\prime} n\right) / \sigma_{n}\right] /\left[\sigma_{A}\left(e, e^{\prime} p\right) / \sigma_{p}\right]=1\right)$ for high-momentum nucleons. 
This confirms that there are equal numbers of high-initial momentum protons and neutrons in asymmetric nuclei, even though these nuclei contain up to 50\% more neutrons than protons. This observation is consistent with high-initial momentum nucleons belonging primarily to $n p$-pairs, even in neutron-rich nuclei.

The surprising fact that increasing the number of neutrons in a nucleus increases the fraction of high-initial momentum protons, which implies that protons have larger average kinetic energy than neutrons, has several broad implications. Neutron stars contain about 5-10\% protons and electrons. Our results imply that the extreme neutron excess in a neutron star could mean a dramatically increase in the protons momentum compared to estimation based on simple Fermi gas. This could affect the cooling rate and equation of state of neutron stars.

There are experimental indications that the high-momentum nucleons associated with SRC pairs are responsible for the EMC effect [39 47]. The latter is a change in the quark momentum distribution in nucleons bound in nuclei compared to free nucleons [39]. The EMC effect (named after the European Muon Collaboration) may be obtained mainly with SRC pairs which can be viewed as temporary high-nuclear-density fluctuations. In these nuclear pairs, the nuclear wavefunctions strongly overlap. The internal structure of the affected nucleons is briefly modified. If this mechanism indeed occurs, then the average proton in neutron-rich nuclei (the minority species) is more likely to belong to a correlated pair and should therefore be more modified than the average neutron (the majority species). Observing such increased modification of the proton structure in neutron-rich nuclei could shed new light on the currently unknown origin of these modifications of nuclear parton-distribution functions.

Furthermore, the observed $n p$-dominance of SRC pairs in heavy nuclei has considerable implications in many areas of nuclear and particle physics, These include nuclear correlation functions and the double-beta decay rate of nuclei [48, the nature of the repulsive core of the nucleon-nucleon interaction 44, 49, 32 and neutrino-nucleus interactions. In the latter the high-precision extraction of oscillation parameters and searches for new physics beyond the Standard Model require detailed understanding of the nuclear ground state and neutrino interaction operators [50].

We also studied the $n p$-dominance directly by triple coincidence measurements of the $A\left(e, e^{\prime} n p\right)$ and $A\left(e, e^{\prime} p p\right)$ reactions. From these measurements we extracted the reduced cross-section ratio for a proton-proton to proton-neutron knockout. We found that the ratio is very small, consistent with previous measurements off symmetric nuclei [6]. Accounting for FSI, using model-dependent SCX corrections, we also extracted the relative abundance of $p p$ - to $n p$-SRC pairs in the measured nuclei. As expected, the SCX corrections lead to a systematic reduction in the $p p$-to- $n p$ ratios (see App. A), making the reported reduced cross-sections an upper limit to the relative SRC pairs abundance ratios. See Fig. 71. 


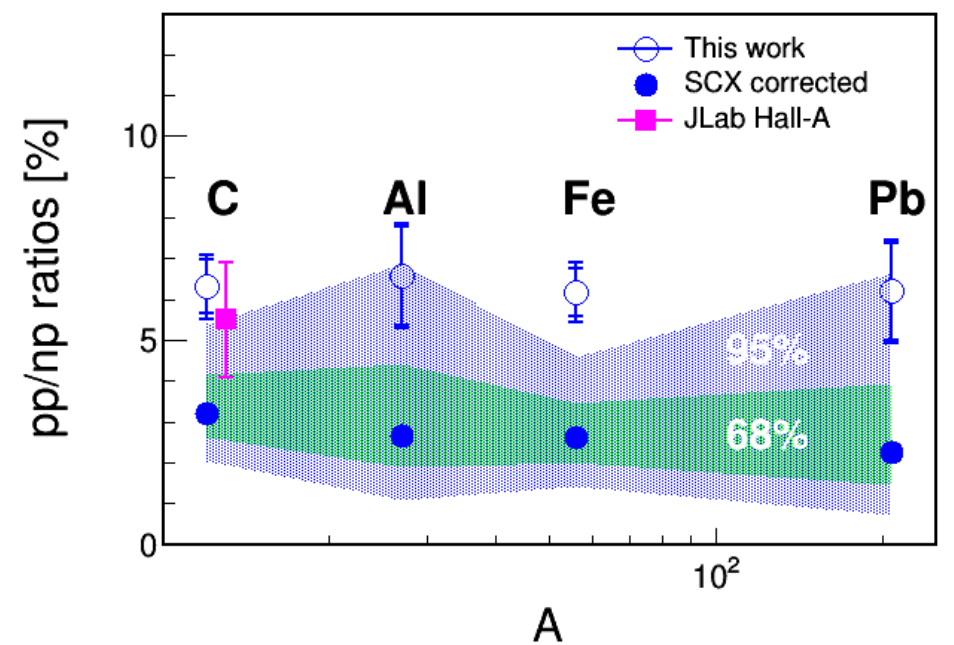

Figure 71: Extracted ratios of $p p$ - to $n p$-SRC pairs in nuclei. The open symbols show the measured reduced crosssection ratios. The filled symbols show the extracted ratios of $p p$ - to $n p$-SRC pairs obtained from the measured cross-section ratios after SCX corrections. The magenta square shows the data of [27, which were also corrected for SCX.

Previous work [6] measured $A\left(e, e^{\prime} p\right)$ and $A\left(e, e^{\prime} p p\right)$ events and derived the relative probabilities of $n p$ and $p p$ pairs assuming that all high-missing momentum $A\left(e, e^{\prime} p\right)$ events were due to scattering off SRC pairs. The agreement between the $p p / n p$ ratios directly measured here and those of the previous indirect measurement, strengthens the $n p$-pair dominance assumption and also lends credence to the previous assumption that almost all high-initial-momentum protons belong to SRC pairs in nuclei from ${ }^{12} \mathrm{C}$ to ${ }^{208} \mathrm{~Pb}\left[6\right.$. This was previously only directly measured in ${ }^{4} \mathrm{He}$ and ${ }^{12} \mathrm{C}$ 49, 27.

Finally, from the determined $A\left(e, e^{\prime} p\right)$ and $A\left(e, e^{\prime} n\right)$ cross-section ratios of nucleus $A$ relative to ${ }^{12} \mathrm{C}$, in both $\mathrm{MF}$ and SRC kinematics, we extracted the nuclear transparency ratios for protons and neutrons. The nuclear transparency of single nucleon knockout reactions is an important quantity for obtaining information about the structure and dynamics of individual nucleons bound in nuclei. Moreover, in this work, when extracting the different ratios of neutron to protons, and proton-proton to neutron-proton, we assumed that the transparency of the knockout nucleon is the same for proton and neutron within the experimental uncertainties (see App. A). This assumption was based on theoretical considerations only, since until this work, measurements of nuclear transparency were performed only for protons and not for neutrons.

The extracted neutron-transparency ratios are consistent with each other for the two measured kinematical regions and agree, within experimental uncertainties, with the proton transparencies extracted from new and previous $\left(e, e^{\prime} p\right)$ measurements, including those from neutron-rich nuclei such as lead. The nuclear-mass-dependence of the extracted transparencies scales as $A^{\alpha}$ with $\alpha=-0.289 \pm 0.007$, which is overall consistent with $-1 / 3$, the value expected for nuclear-surface dominance of the reactions. Moreover, the proton knockout data agree with Glauber calculations, validating their use in analyses of high-energy nuclear reactions. While Galuber calculations of neutron knockout reactions are unavailble at the moment, they should not signicantly deviate from the proton knockout calcualtions in order to be consistent with the data presented here. 
The results presented in Figs. 69,70 were published recently in Nature (M. Duer et al. (CLAS Collaboration), Nature, 506, 617 (2018)). They also serve as the base for another publication resulted from this analysis: B. Schmookler, M. Duer et al. (CLAS Collaboration), accepted for publication in Nature (December 2018). Two more papers, based on the latter two results discussed above, were submitted for Phys. Rev. Lett., and are being reviewed at the time this thesis was submitted:

- M. Duer et al. (CLAS Collaboration), "Direct Observation of Proton-Neutron Short-Range Correlation Dominance in Heavy Nuclei", https://arxiv.org/pdf/1810.05343.pdf

- M. Duer et al. (CLAS Collaboration), "Measurement of Nuclear Transparency Ratios for Protons and Neutrons", https://arxiv.org/pdf/1811.01823.pdf 


\section{References}

[1] J. Chadwick, Proc. R. Soc. Lond. A136 (1932).

[2] G. Mayer, Phys. Rev. 74, 235 (1948).

[3] S. Frullani, and J. Mougey, Adv. Nucl. Phys. 14, 1-283 (1984).

[4] L. Lapikas, Nucl. Phys. A 553, 297 (1993).

[5] B. A. Mecking, et al., Nucl. Instr. Meth., A 503, 512 (2003).

[6] O. Hen et al. (CLAS Collaboration), Science 346, 614 (2014).

[7] O. Hen, E. Piasetzky, and L. B. Weinstein, Phys. Rev. C 85, 047301 (2012).

[8] L. El Fassi et al. (CLAS Collaboration), Phys. Lett. B 712, 326 (2012).

[9] M. D. Mestayer, et al., Nucl. Inst. and Meth. A 449, 81 (2000).

[10] G. Adams, V. Burket, et al., Nucl. Instr. and Meth., A 465, 414 (2001).

[11] E. S. Smith, et al., Nucl. Instr. and Meth., A 443, 265 (1999).

[12] M. Amarian, et al., Nucl. Instr. and Meth., A 460, 239 (2001).

[13] J. Lachniet et al., "A high precision measurement of the neutron magnetic form factor using the CLAS detector", approved CLAS analysis note (2008).

[14] O. Hen et al., "PROBING PP-SRC IN ${ }^{12} \mathrm{C},{ }^{27} \mathrm{Al},{ }^{56} \mathrm{Fe}$, AND ${ }^{208} \mathrm{~Pb}$ USING THE $A\left(e, e^{\prime} p\right)$ AND $A\left(e, e^{\prime} p p\right)$ REACTIONS", approved CLAS analysis note (2012).

[15] M. Duer et al., "PROBING $2 \mathrm{~N}-\mathrm{SRC}$ in ${ }^{12} \mathrm{C},{ }^{27} \mathrm{Al},{ }^{56} \mathrm{Fe}$, and ${ }^{208} \mathrm{~Pb}$ using the $A\left(e, e^{\prime} n\right)$ and $A\left(e, e^{\prime} p\right)$ reactions" , approved CLAS analysis note (2017).http://www-nuclear.tau.ac.il/ eip/neutron3.pdf

[16] T.G. Oneill et al., Phys. Lett. B 87, 351 (1995).

[17] D. F. Geesaman et al., Phys. Rev. Lett. 63, 734 (1989).

[18] G. Garino et al., Phys. Rev. C 45, 780 (1992).

[19] C. Ciofi degli Atti, S. Simula, Phys. Rev. C 53, 1689 (1996).

[20] E. Wolin, GSIM Users Guide Version CERN 1.0, 1995.

[21] L. B. Weinstein and S. E. Kuhn, Short distance structure of nuclei: Mining the wealth of existing Jefferson Lab data, DOE Grant DE-SC0006801 (2016).

[22] H. Hakobyan et al., Nucl. Inst. and Meth. A 592, 218-223 (2008).

[23] M. Sargsyan, "Computer Code for Inclusive (e,e') Electro-production Reactions and Radiative Corrections", CLAS-NOTE 90-007, (1990).

[24] O. Hen et al. (CLAS Collaboration), Phys. Lett. B 722, 63 (2013).

[25] W. P. Ford, S. Jeschonnek and J. W. Van Orden, "Momentum distributions for ${ }^{2} H\left(e, e^{\prime} p\right)$ ", arXiv:1411.330611 [nucl-th] (2014). 
[26] M. Duer et al. (CLAS Collaboration), Nature, 506, 617 (2018).

[27] R. Subedi et al., Science 320, 1476 (2008).

[28] C. Colle, W. Cosyn, and J. Ryckebusch, Phys. Rev. C93, 034608 (2016).

[29] J. Ryckebusch, D. Debruyne, P. Lava, S. Janssen, B. Van Overmeire, and T. Van Cauteren, Nucl. Phys. A728, 226 (2003).

[30] T. De Forest, Nucl. Phys. A392, 232 (1983).

[31] L. Frankfurt, M. Strikman, and M. Zhalov, Phys. Lett. B503 73 (2001).

[32] B. Schmookler et al. (CLAS Collaboration), accepted to Nature (2018).

[33] D. Abbott et al., Phys. Rev. Lett. 80, 5072 (1998).

[34] K. Garrow et al., Phys. Rev. C66, 044613 (2002).

[35] V. R. Pandharipande and S. C. Pieper, Phys. Rev. C45, 791 (1992).

[36] W. Cosyn, Private communication.

[37] R. B. Wiringa, R. Schiavilla, S. C. Pieper, J. Carlson, Phys. Rev. C 89, 024305 (2014).

[38] M. Sargsian, Phys. Rev. C 89, 034305 (2014).

[39] L. B. Weinstein, E. Piasetzky, D. W. Higinbotham, J. Gomez, O. Hen, \& R. Shneor, Phys. Rev. Lett. 106, 052301 (2011).

[40] J. O’Meara et al., IEEE Trans. Mag. 25 (2), 1902 (1989).

[41] Program Library Long Writeup W5013 Copyright CERN. Geneva, GEANT, 1993.

[42] G. P. Gilfoyle, M. Ito, and E. Wolin, Online RECSIS, CLAS-Note 98-017.

[43] L. Frankfurt, M. Sargsian, and M. Strikman, International Journal of Modern Physics A 23, 2991 (2008).

[44] C. Ciofi degli Atti, Phys. Rept. 590, 1 (2015).

[45] L. Frankfurt, M. Sargsian, and M. Strikman, Phys. Rev. C56, 1124 (1997).

[46] M. Sargsian, Int. J. Mod. Phys. E10, 405 (2001).

[47] O. Hen, , G. A. Miller, E. Piasetzky, \& L. B. Weinstein, Rev. Mod. Phys. 89, 045002 (2017).

[48] M. Kortelainen, and J. Suhonen, Phys. Rev. C 76, 024315 (2007).

[49] I. Korover et al., Phys. Rev. Lett. 113, 022501 (2014).

[50] H. Gallagher, G. Garvey, \& G. P. Zeller, Annu. Rev. Nucl. Part. Sci. 61, 355-378 (2011).

[51] K. Egiyan et al., Phys. Rev. C68, 014313 (2003).

[52] L. Frankfurt, M. Strikman, D. Day and M. Sargsian, Phys. Rev. C 48 (1993) 2451.

[53] K. Egiyan et al., Phys. Rev. Lett 96 (2006) 082501.

[54] N. Fomin et al., Phys. Rev. Lett. 108 (2012) 092502. 
[55] A. Tang et al., Phys. Rev. Lett. 90 (2003) 042301.

[56] E. Piasetzky et al., Phys. Rev. Lett. 97 (2006) 162504.

[57] R. Shneor et al., Phys. Rev. Lett. 99 (2007) 072501.

[58] M. Sargsian, T. Abrahamyan, M. Strikman and L. Frankfurt, Phys. Rev. C 71 (2005).

[59] R. Schiavilla, R. B. Wiringa, S. C. Pieper and J. Carlson, Phys. Rev. Lett. 98 (2007).

[60] M. Vanhalst, J. Ryckebusch, Wim Cosyn, Phys. Rev. C 86, 044619 (2012).

[61] R. J. Furnstahl, B. D. Serot, H. B. Tang, Nucl. Phys. A 615, 441 (1997).

[62] R.B. Wiringa, V. G. J. Stoks, R. Schiavilla, Phys. Rev. C 51, 38 (1995).

[63] E. Moniz et al., Phys. Rev. Lett. 26, 445 (1971).

[64] J. Ryckebusch, J. Phys G42 (2015). 


\section{Appendices}

\section{A ${ }^{27} \mathrm{Al},{ }^{56} \mathrm{Fe}$, and ${ }^{208} \mathrm{~Pb}$ distributions (high-momentum)}

In this section we show distributions presented in Sec. 3.2 .1 for ${ }^{27} \mathrm{Al},{ }^{56} \mathrm{Fe}$, and ${ }^{208} \mathrm{~Pb}$.

Figures 72,74 show the false positive and negative probabilities (same as Fig. 41).
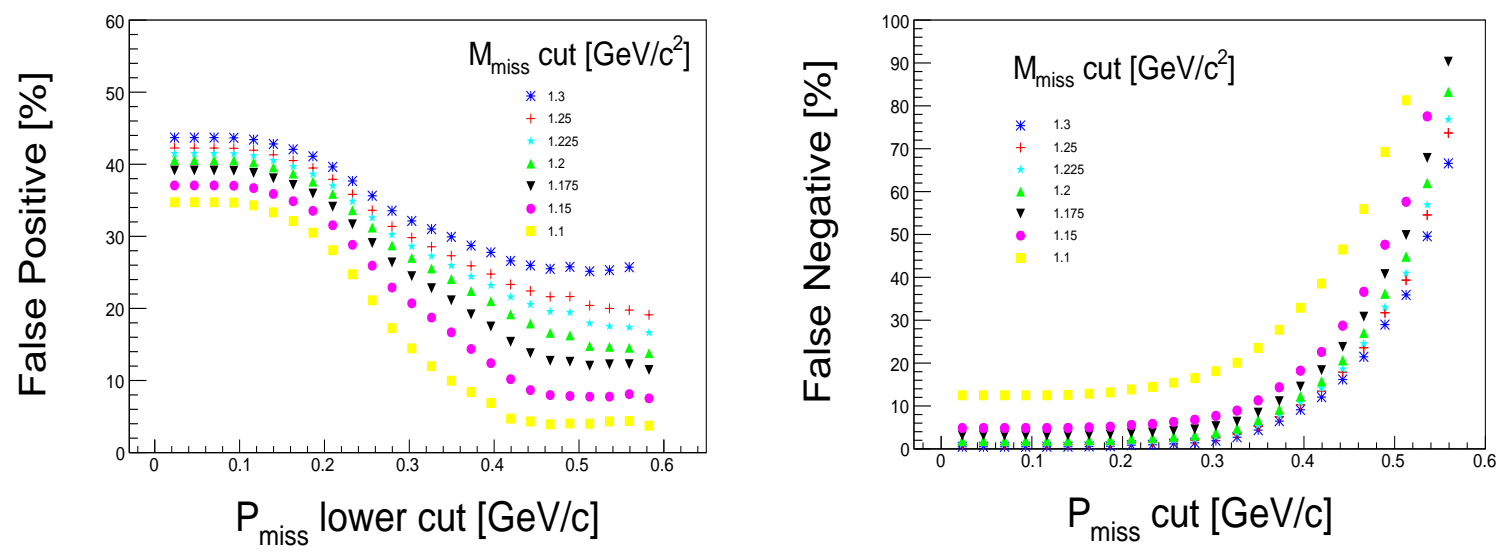

Figure 72: The false positive (left) and negative (right) probabilities for ${ }^{27} \mathrm{Al}$.
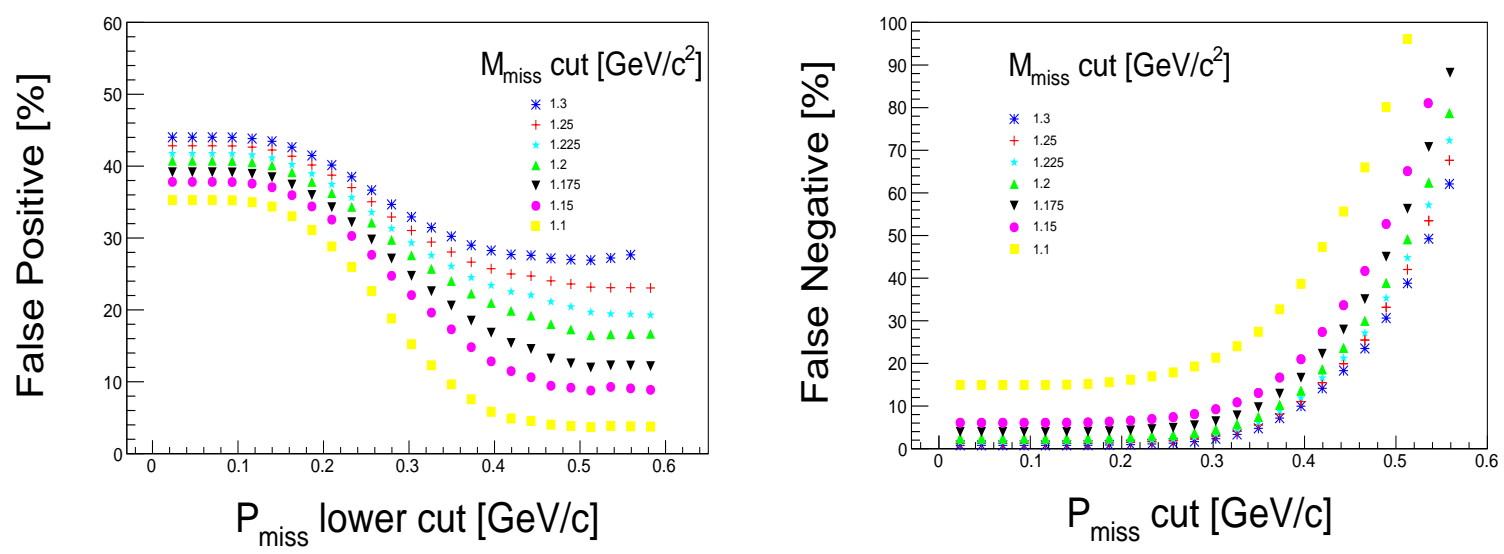

Figure 73: Same as Fig. 72 for ${ }^{56} \mathrm{Fe}$. 

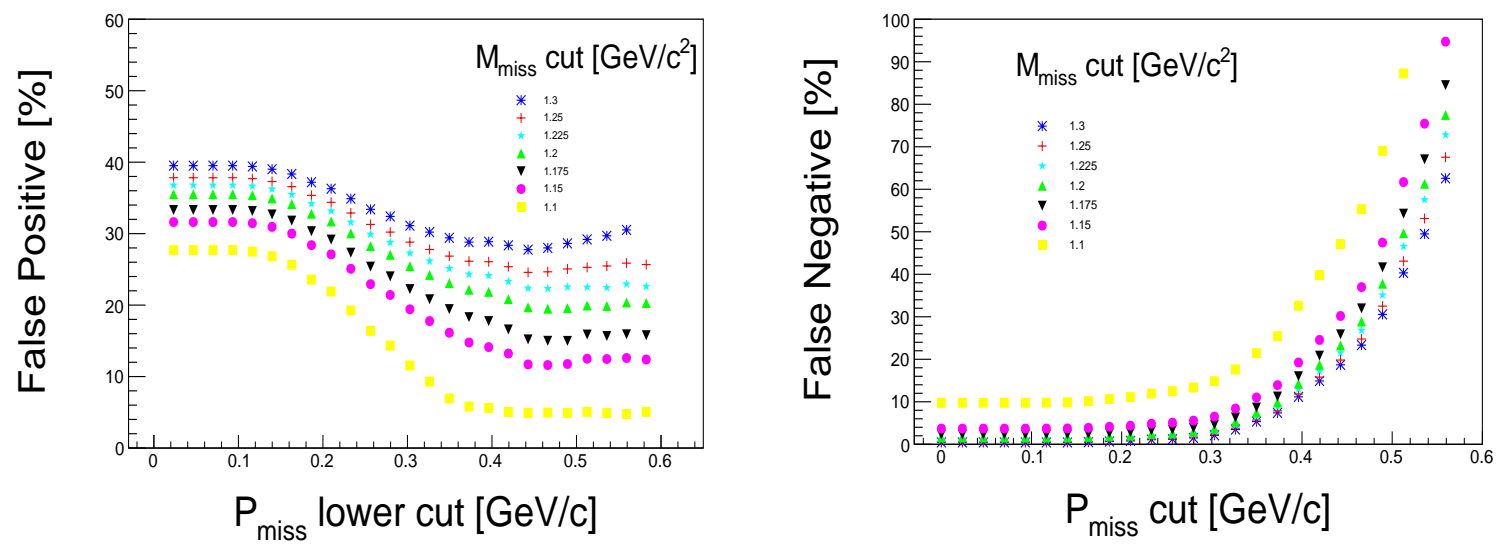

Figure 74: Same as Fig. 72 for ${ }^{208} \mathrm{~Pb}$.

Figures 75,76 show the electron and smeared proton kinematics for the selected $\left(e, e^{\prime} p_{\text {smeared }}\right)$ events (same as Figs. 42,43). 
(a)

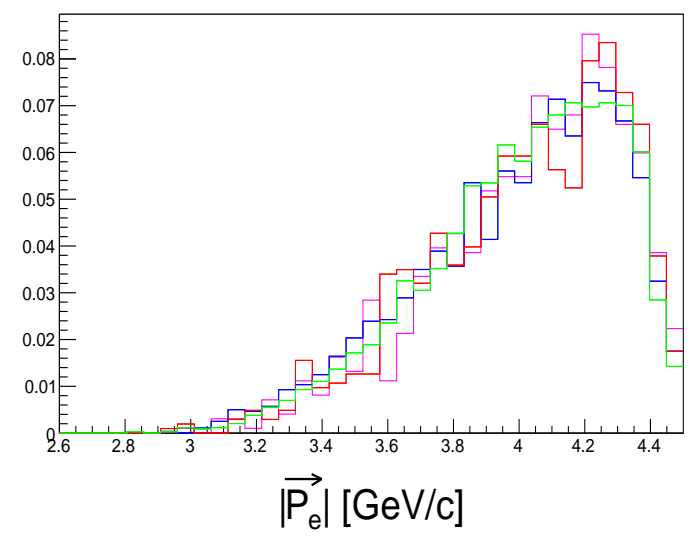

(c)

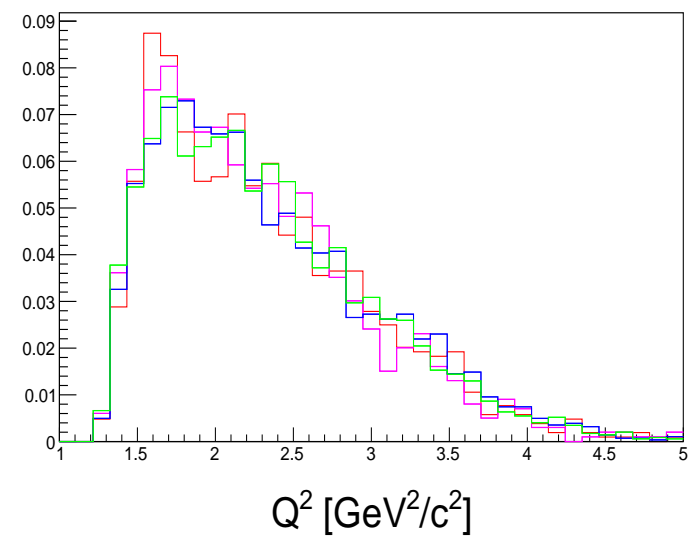

(b)

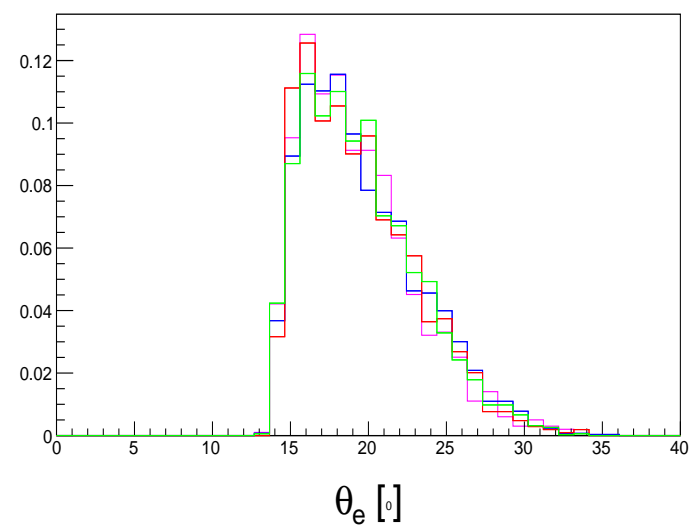

(d)

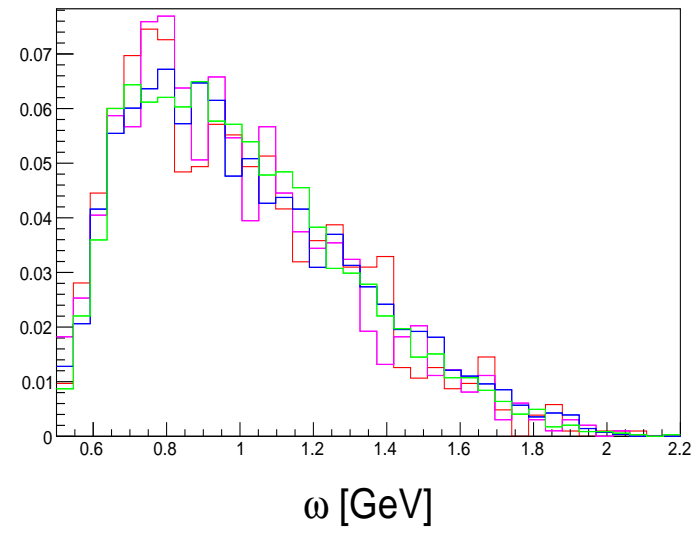

(e)

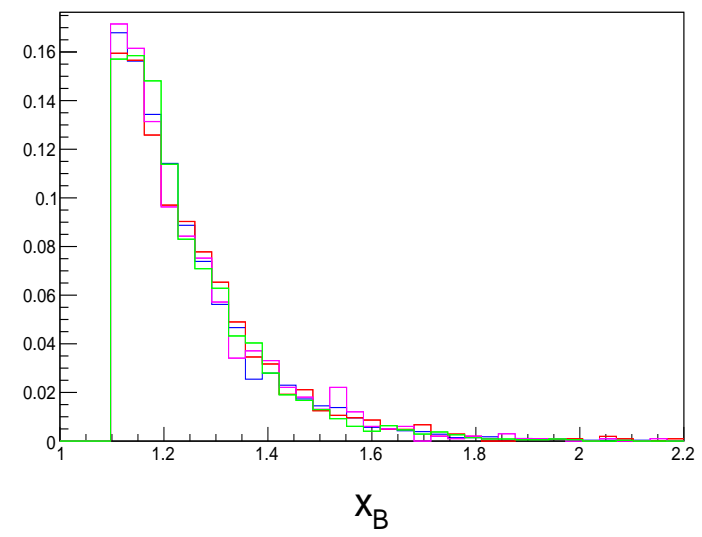

Figure 75: Electron kinematics variables for the selected $\left(e, e\right.$ p $p_{\text {smeared }}$ ) events from ${ }^{12} \mathrm{C}$ (blue), ${ }^{27} \mathrm{Al}(\mathrm{red}),{ }^{56} \mathrm{Fe}$ (green), and ${ }^{208} \mathrm{~Pb}$ (pink): (a) the electron momentum, (b) the electron scattering angle, (c) $Q^{2}$ (d) $\omega$, and (e) $x_{B}$. Distributions are normalized to unity such that only their shapes are to be compared. 
(a)

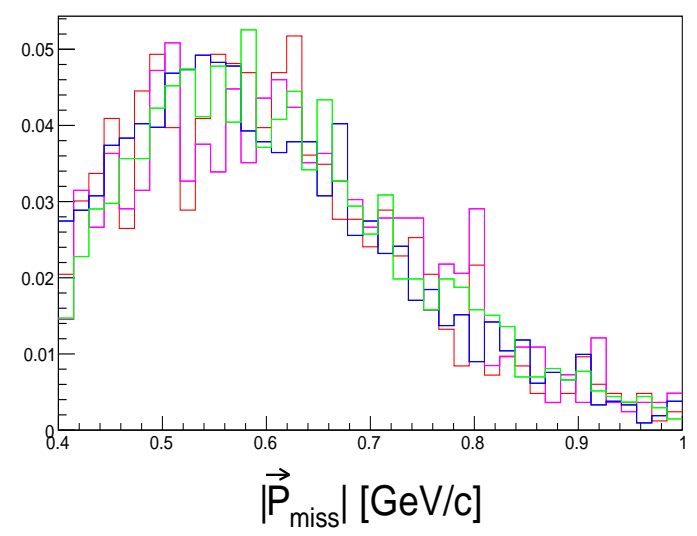

(c)

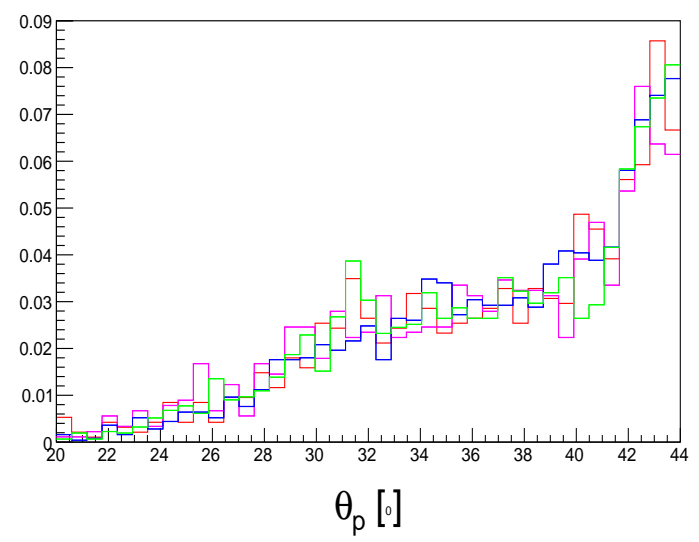

(b)

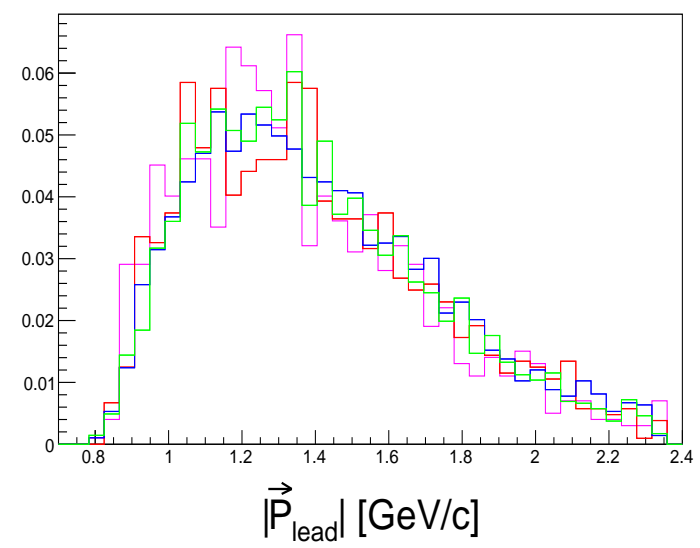

(d)

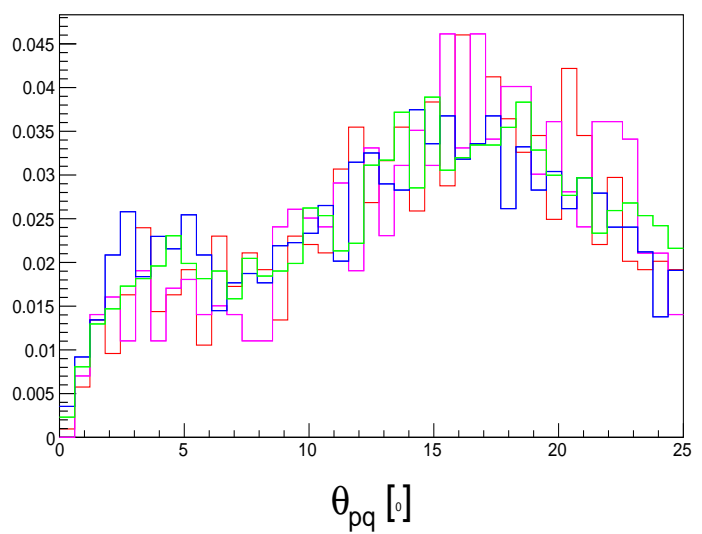

(e)

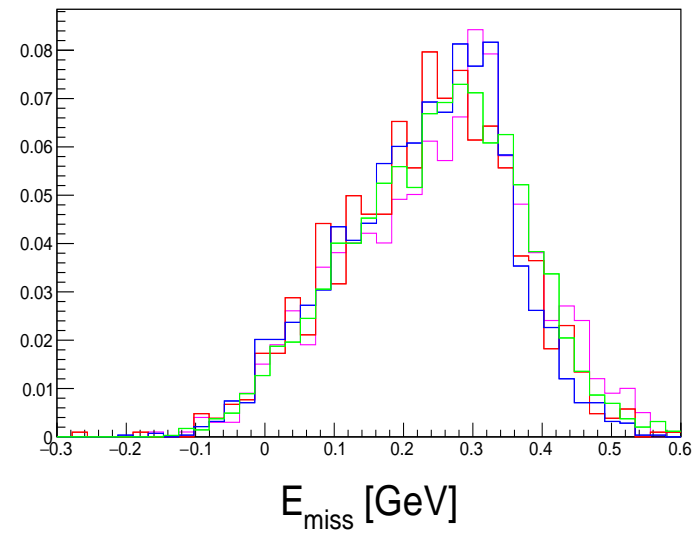

Figure 76: Smeared protons kinematics for the selected $\left(e, e^{\prime} p_{\text {smeared }}\right)$ events from ${ }^{12} \mathrm{C}$ (blue), ${ }^{27} \mathrm{Al}(\mathrm{red}),{ }^{56} \mathrm{Fe}$ (green), and ${ }^{208} \mathrm{~Pb}$ (pink): (a) the missing momentum, (b) the proton momentum, (c) $\theta_{p}(\mathrm{~d}) \theta_{p q}$, and (e) the missing energy. Distributions are normalized to unity such that only their shapes are to be compared. 


\section{B ${ }^{27} \mathrm{Al},{ }^{56} \mathrm{Fe}$, and ${ }^{208} \mathrm{~Pb}$ distributions (low-momentum)}

In this section we show distribtuions presented in Sec. 3.2 .2 for ${ }^{27} \mathrm{Al},{ }^{56} \mathrm{Fe}$, and ${ }^{208} \mathrm{~Pb}$.

Figures 77- 79 shows the missing energy vs. missing momentum for protons and neutrons (same as Fig. 46

(a) protons

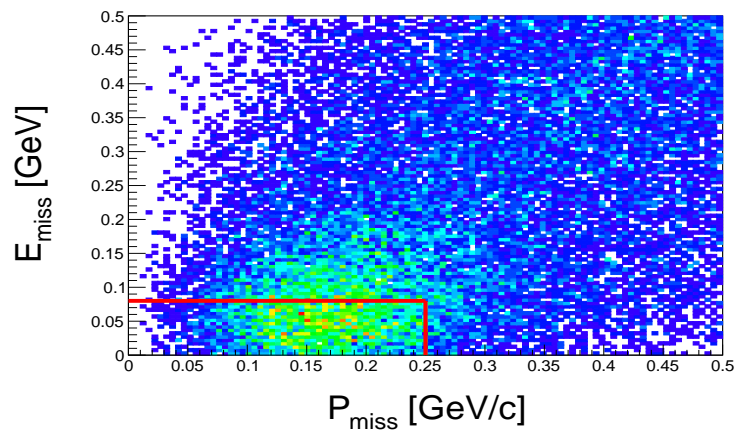

(b) neutrons

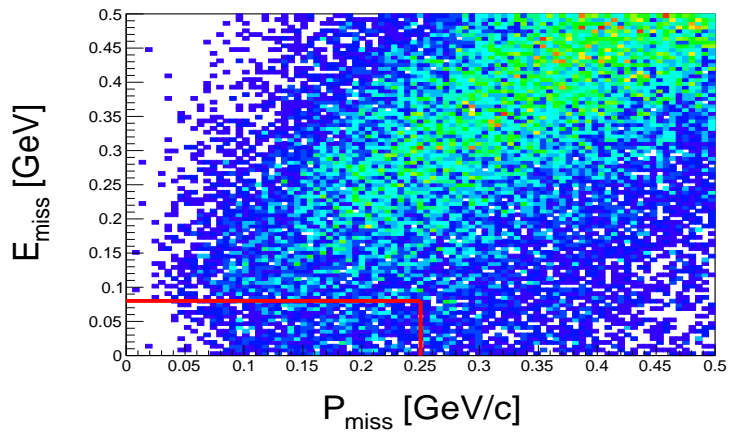

(c) smeared protons

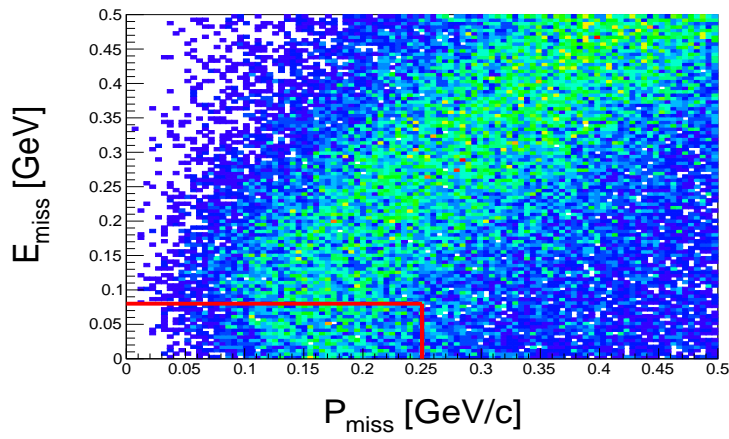

Figure 77: The missing energy vs. the missing momentum for (a) ${ }^{27} \mathrm{Al}\left(e, e^{\prime} p\right)$ data, (b) ${ }^{56} \mathrm{Fe}\left(e, e^{\prime} n\right)$ data, and (c) ${ }^{208} \mathrm{~Pb}\left(e, e^{\prime} p_{\text {smeared }}\right)$ data. The red lines represent the $\mathrm{QE}$ region as defined by $E_{\text {miss }}$ and $P_{\text {miss }}$. 
(a) protons

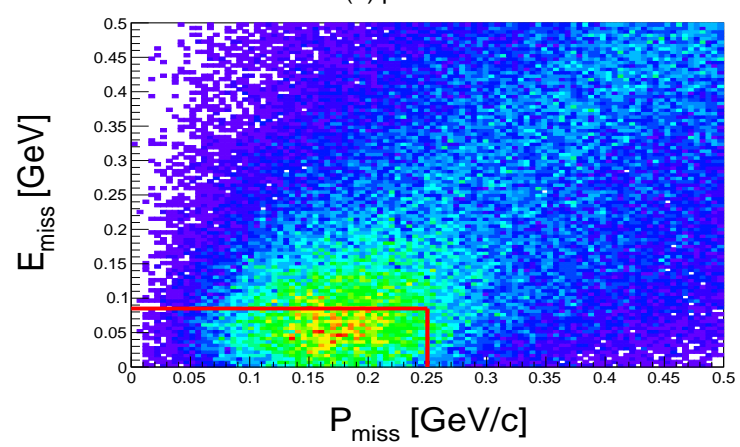

(b) neutrons

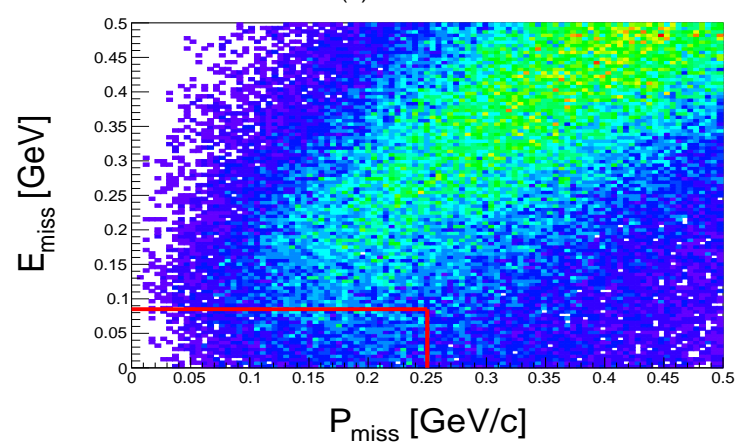

(c) smeared protons

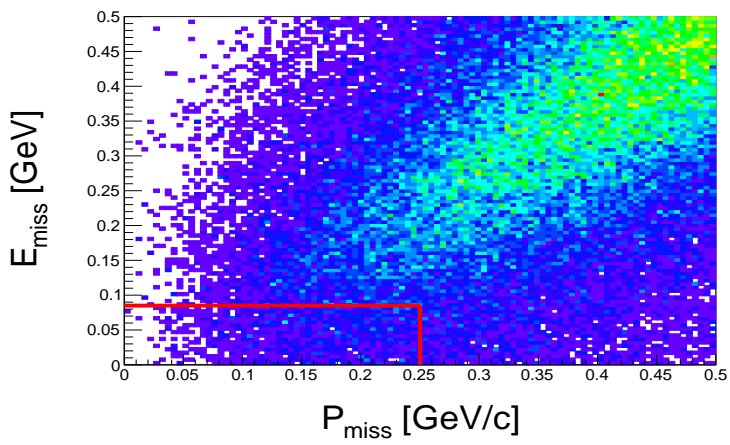

Figure 78: Same as Fig. 77 for ${ }^{56} \mathrm{Fe}$. (a) protons

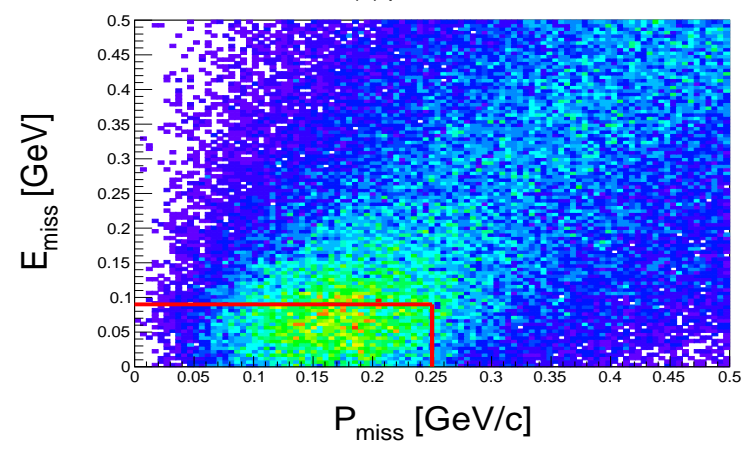

(b) neutrons

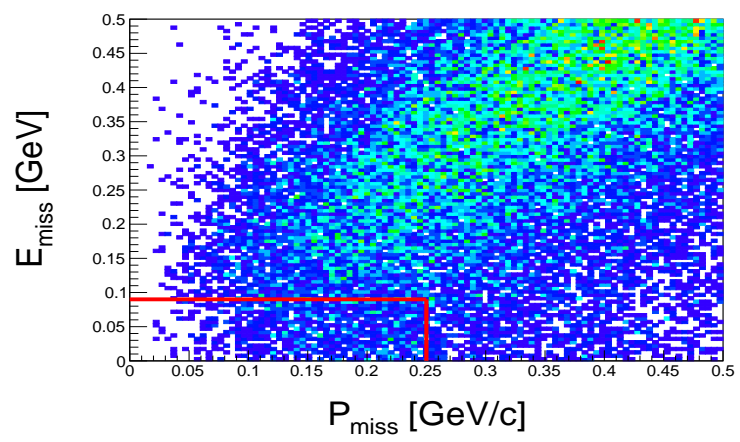

(c) smeared protons

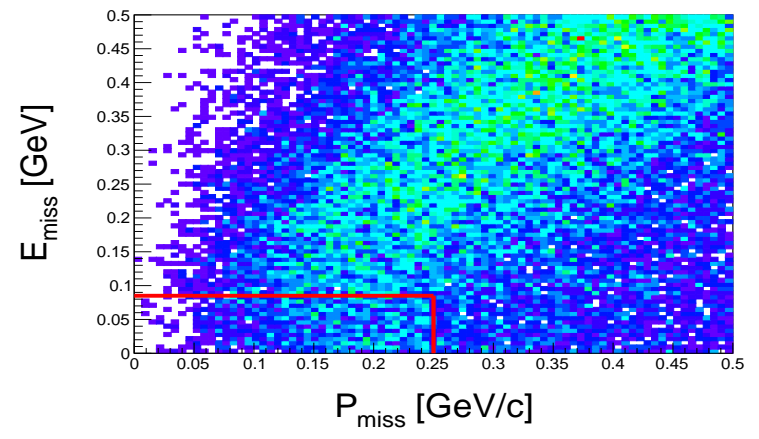

Figure 79: Same as Fig. 77 for ${ }^{208} \mathrm{~Pb}$.

Figures 80 - 82 show the correlations between $y$ and $\omega$, and between $Q^{2}$ and $\theta_{N q}$, the angle between the detected nucleon and the momentum transfer vector (same as Fig. 47). 


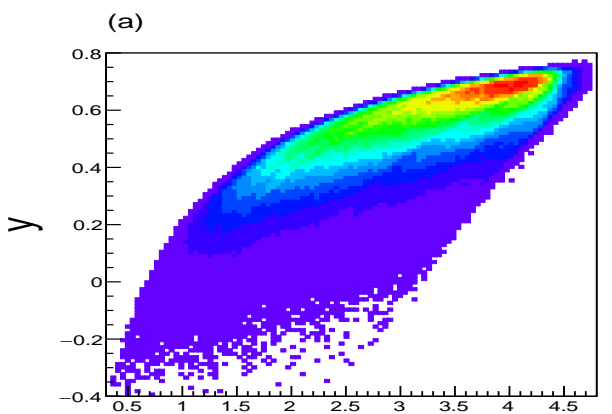

$\omega[\mathrm{GeV}]$

(b)

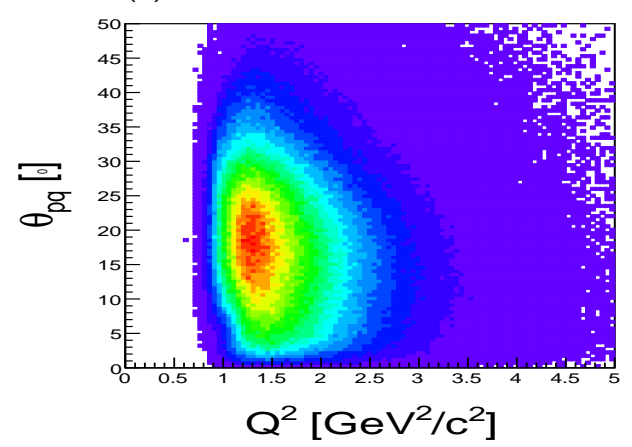

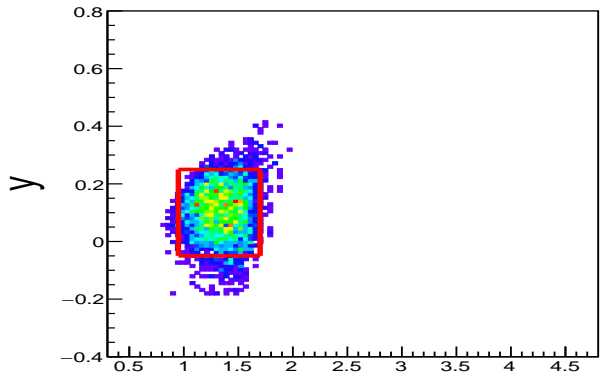

$\omega[\mathrm{GeV}]$

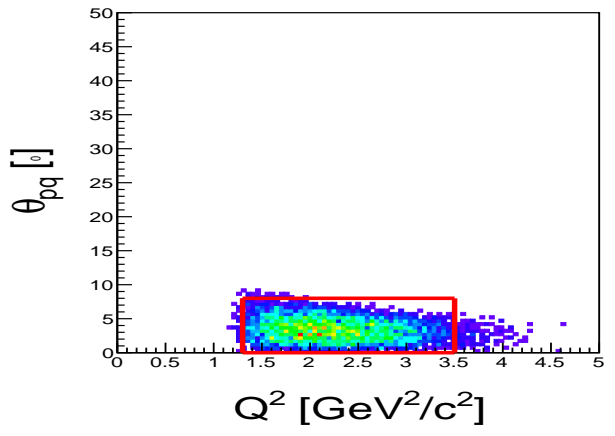

Figure 80: ${ }^{27} \mathrm{Al}$ : (a) The $y$ scaling variable vs. $\omega$ with (right) and without (left) the QE cuts for un-smeared protons. (b) Same as (a) for $\theta_{p q}$ vs. $Q^{2}$.

(a)

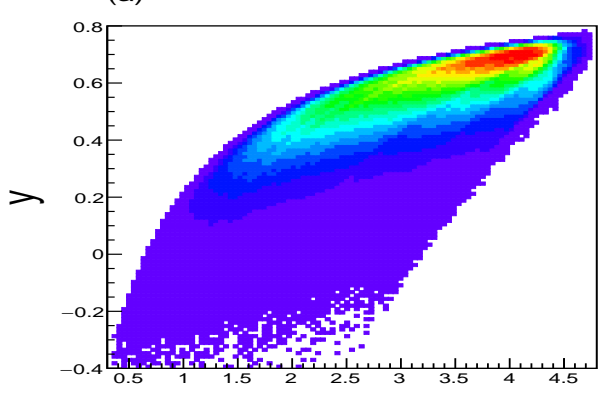

$\omega[\mathrm{GeV}]$

(b)

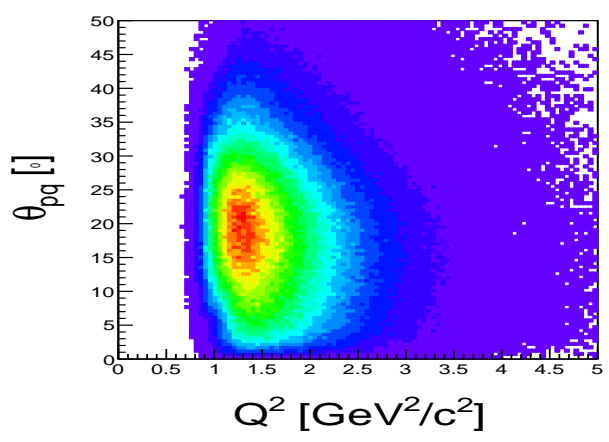

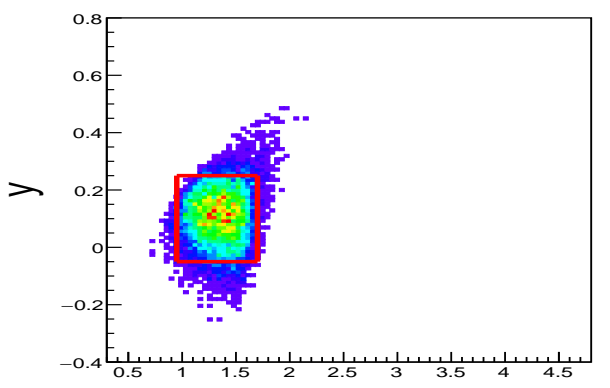

$\omega[\mathrm{GeV}]$

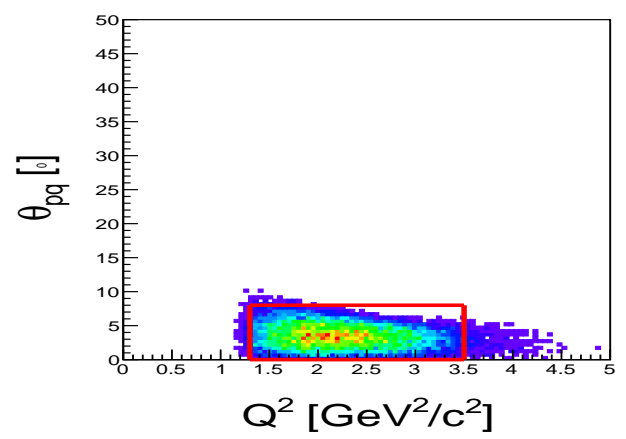

Figure 81: Same as 80 for ${ }^{56} \mathrm{Fe}$. 


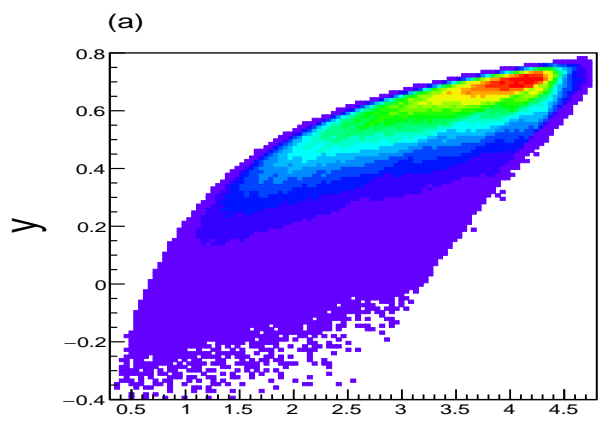

$\omega[\mathrm{GeV}]$

(b)

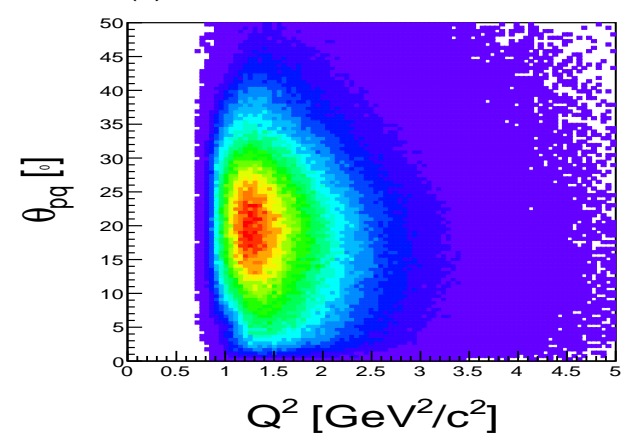

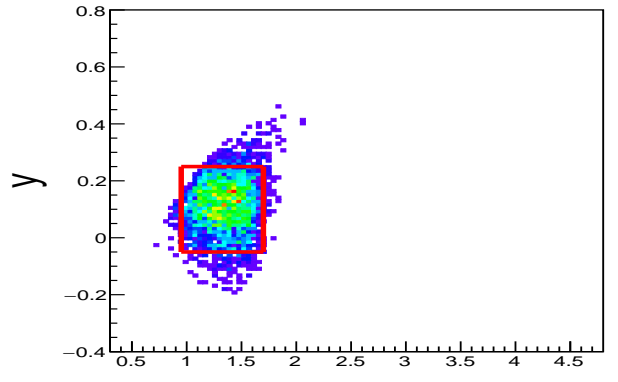

$\omega[\mathrm{GeV}]$

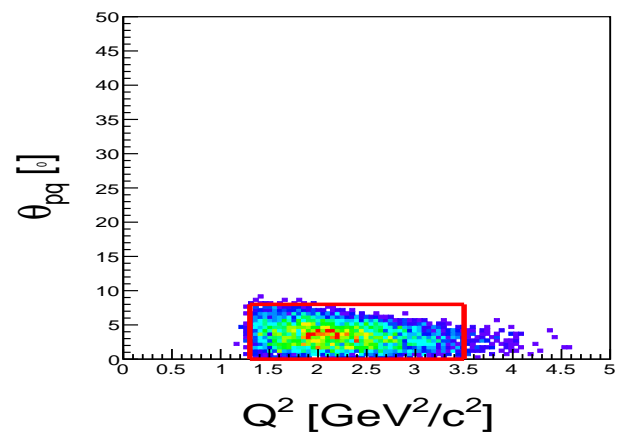

Figure 82: Same as 80 for ${ }^{208} \mathrm{~Pb}$.

Figures 83 - 85 show the false positive and negative probabilities (same as Fig. 48).
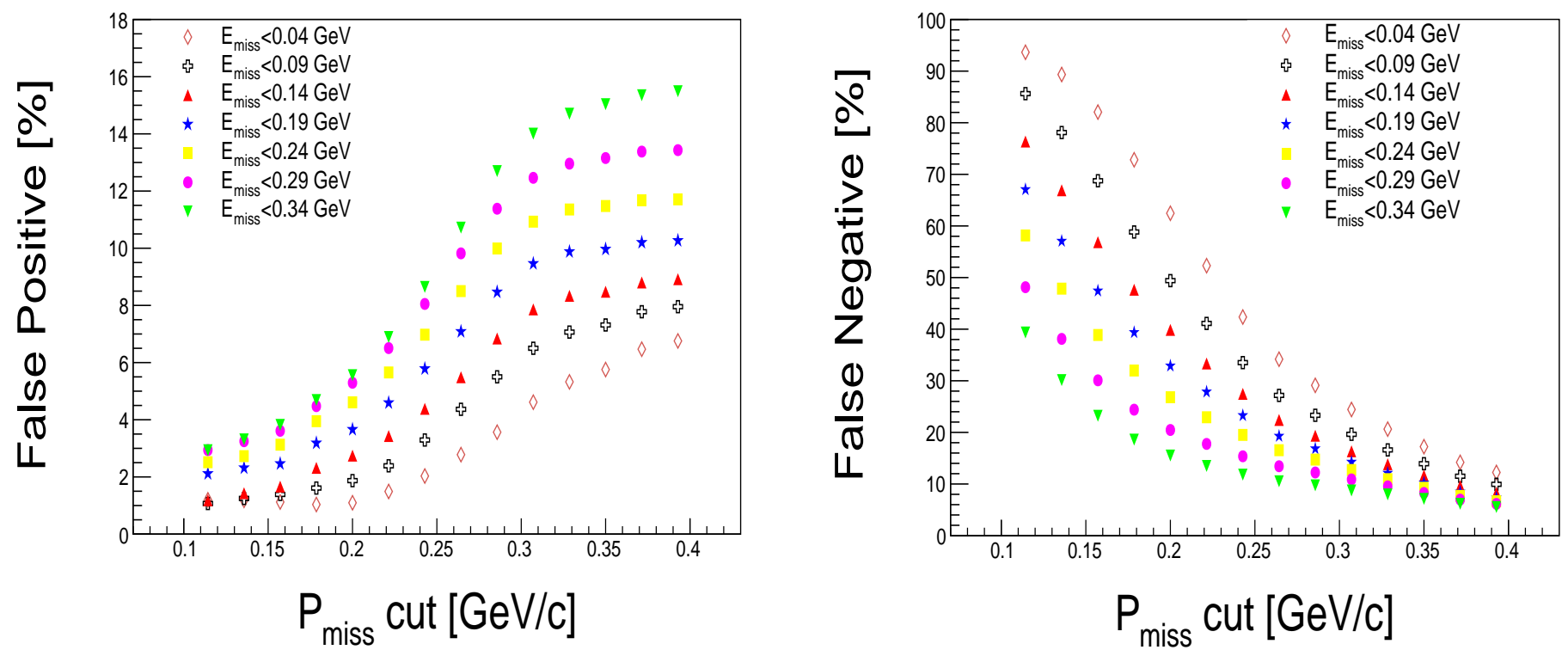

Figure 83: The false positive (left) and negative (right) probabilities for the low missing momentum (QE) events, as a function of the missing momentum cut for different missing energy cuts, for ${ }^{27} \mathrm{Al}$. The blue circle represents the selected cuts. 

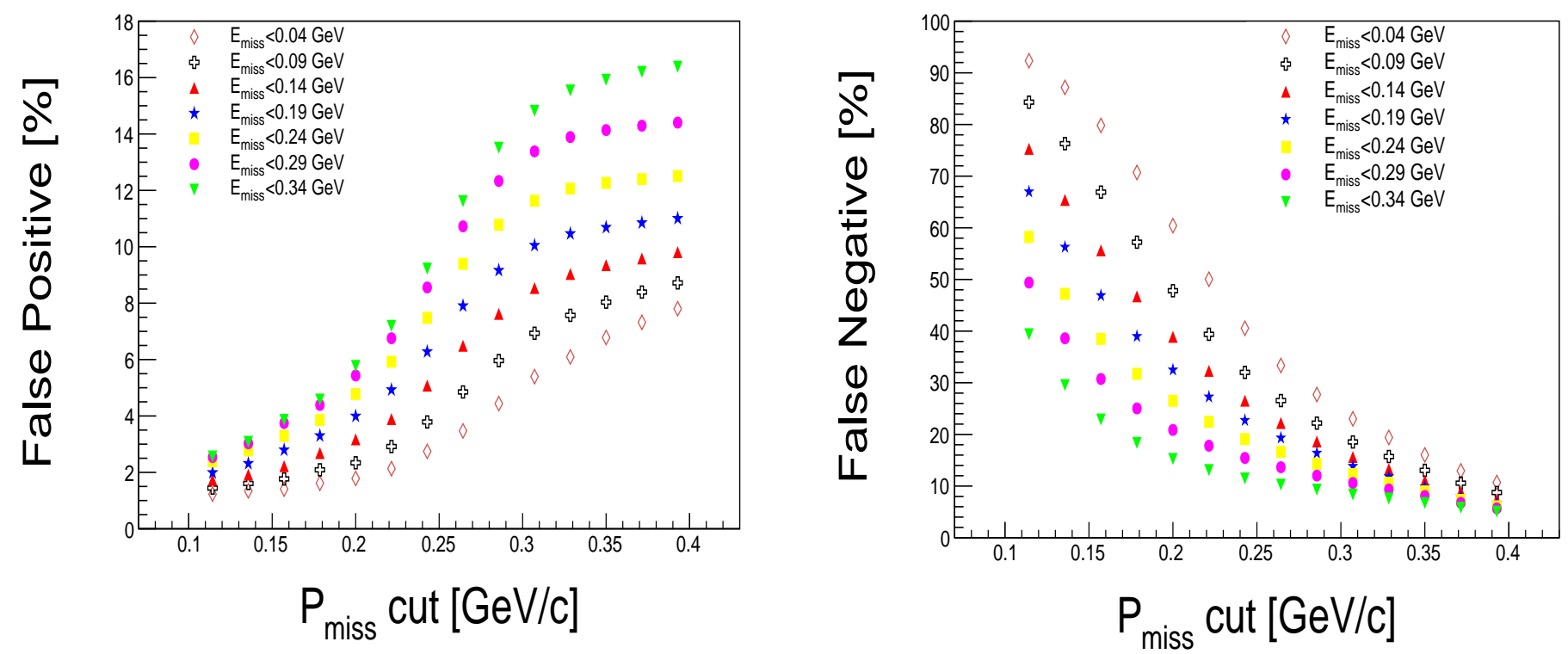

Figure 84: Same as Fig. 83 for ${ }^{56} \mathrm{Fe}$.
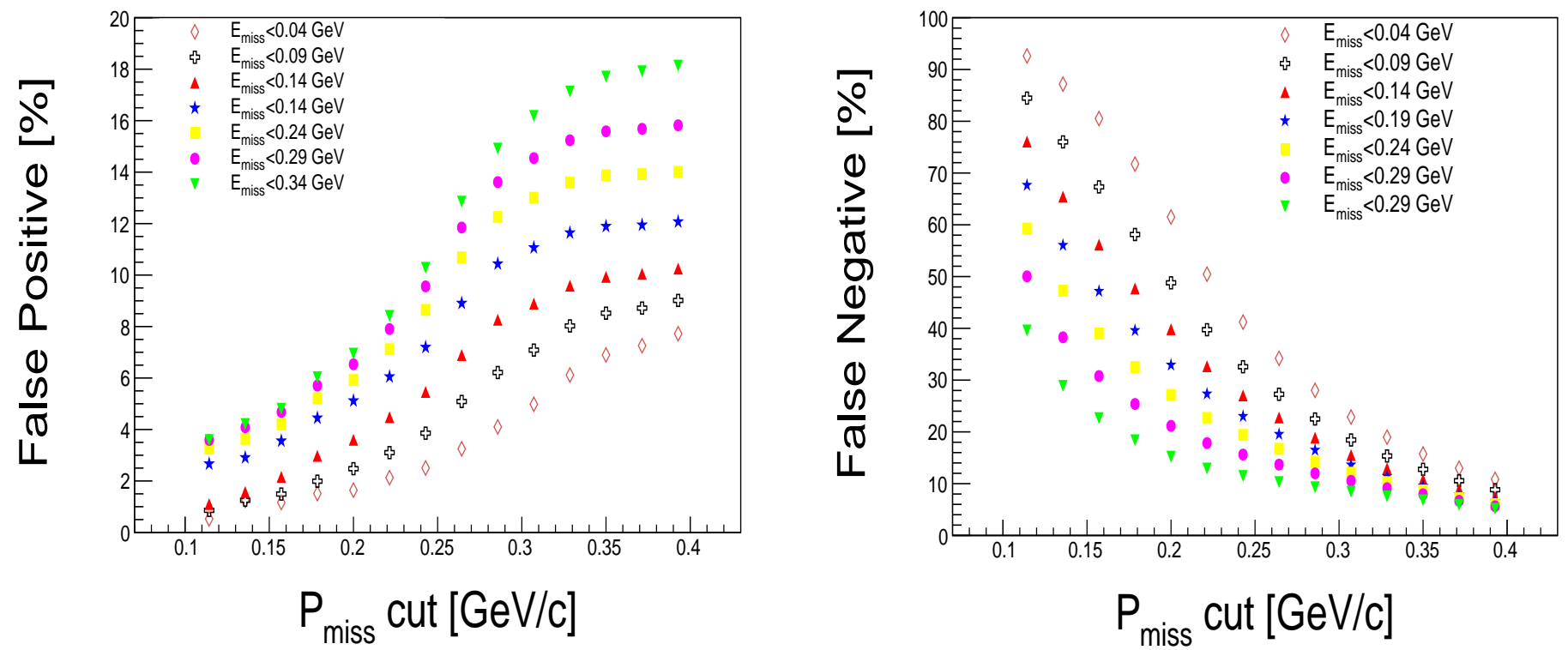

Figure 85: Same as Fig. 83 for ${ }^{208} \mathrm{~Pb}$.

Figures 86 - 88 shows the electron kinematic variables distributions after application of the QE cuts for smeared protons and neutrons (same as Fig. 49). 

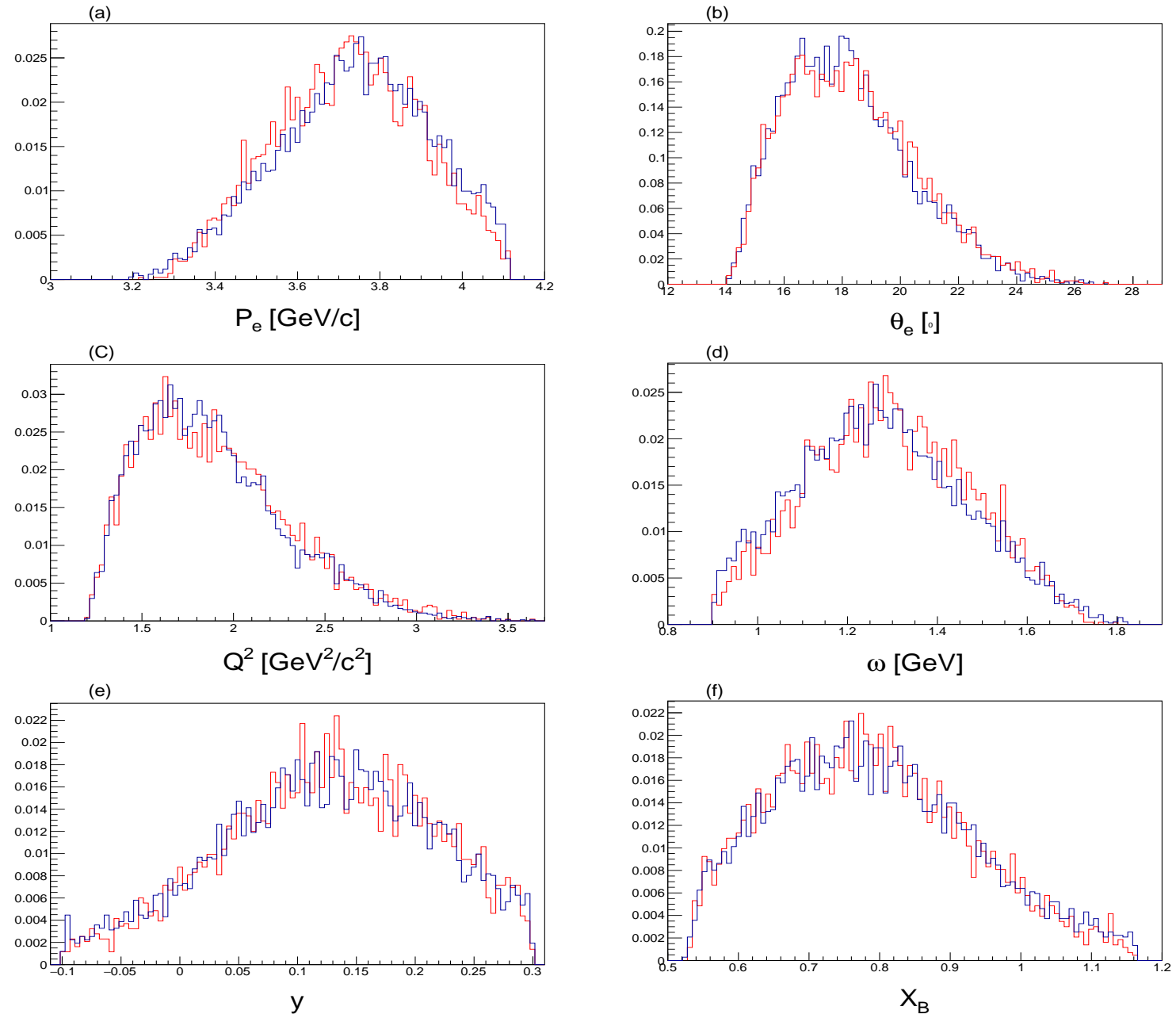

Figure 86: The electron kinematic variables for ${ }^{12} \mathrm{Al}\left(e, e^{\prime} p_{\text {smeared }}\right)$ (red) and ${ }^{12} \mathrm{Al}\left(e, e^{\prime} n\right)$ (blue) after applying the QE cuts: (a) the electron momentum, (b) the electron scattering angle, (c) $Q^{2}$, (d) $\omega$, (e) $y$, and (f) $x_{B}$. All distributions are normalized to unity such that only their shape is compared. 
(a)

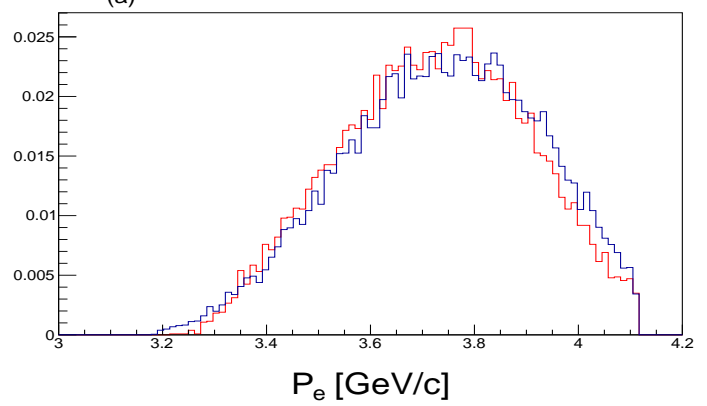

(c)

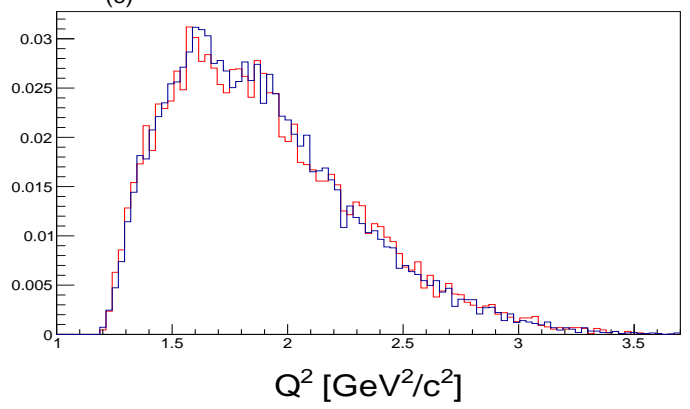

(e)

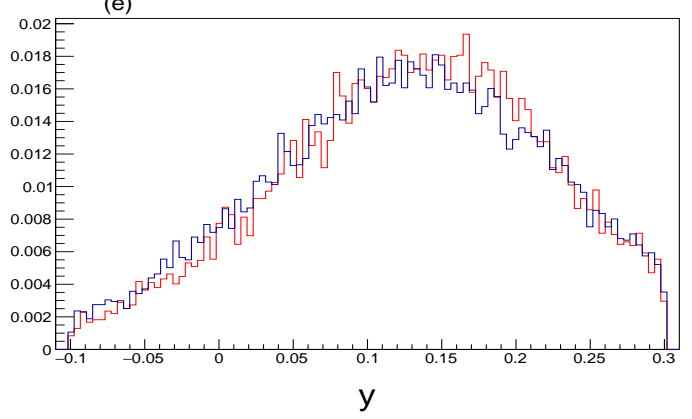

(b)

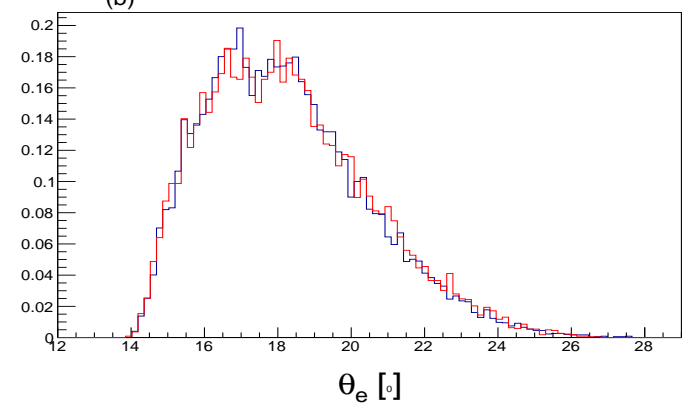

(d)
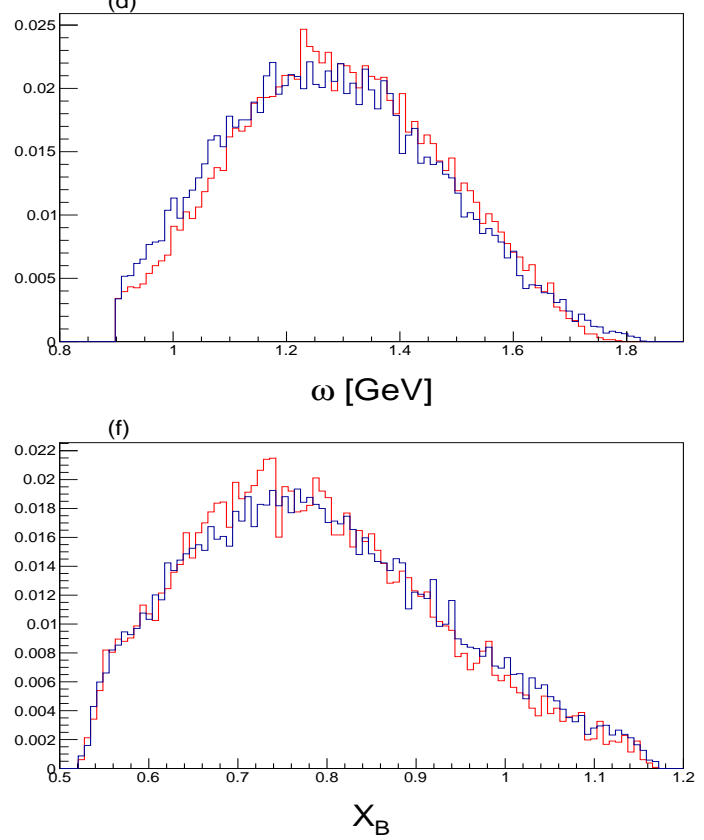

Figure 87: Same as Fig. 86 for ${ }^{56} \mathrm{Fe}$.

100 

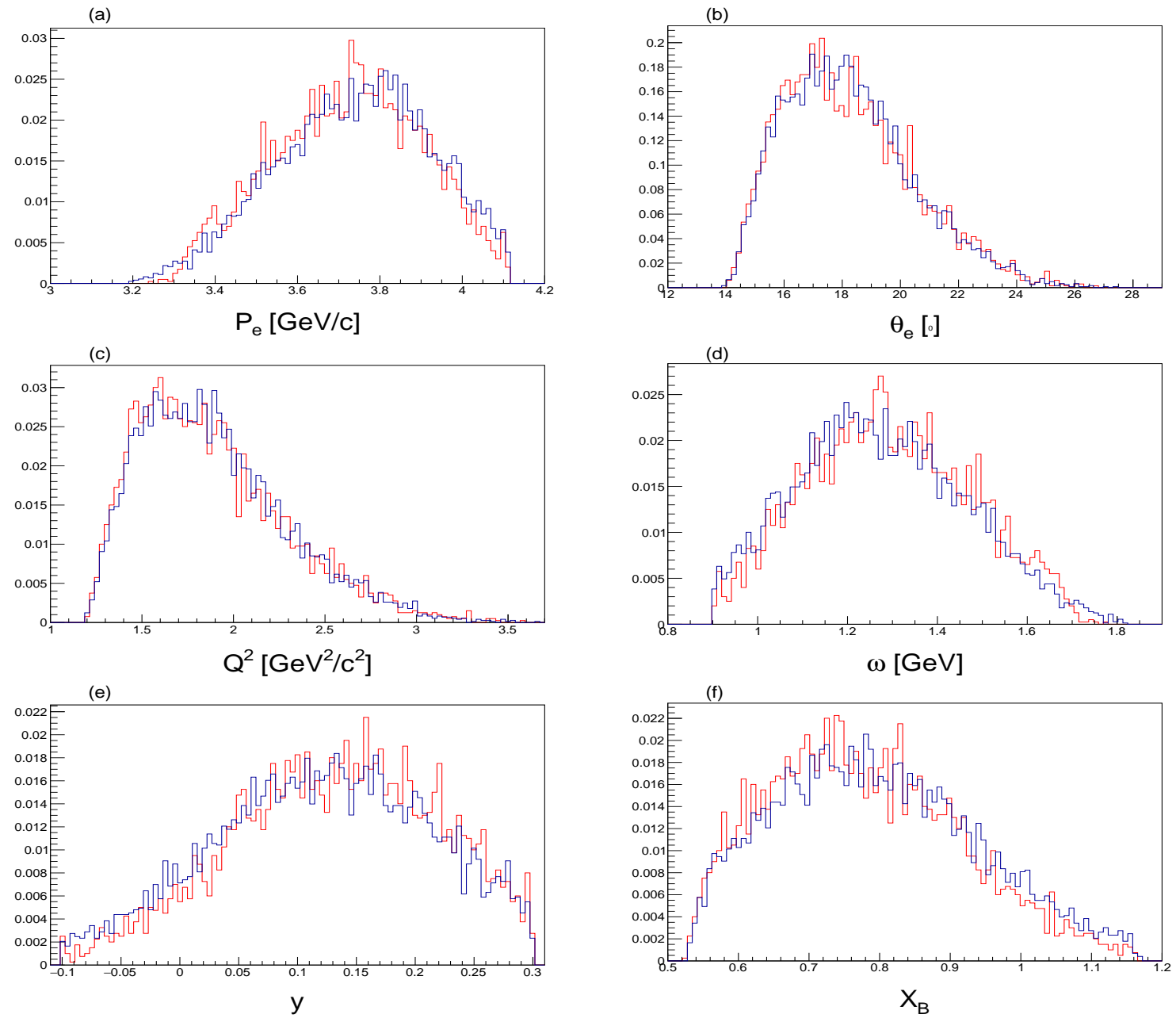

Figure 88: Same as Fig. 86 for ${ }^{208} \mathrm{~Pb}$.

Figures 89-91 show the nucleon kinematic variables distributions after application of the QE cuts for smeared protons and neutrons (same as Fig. 50). 


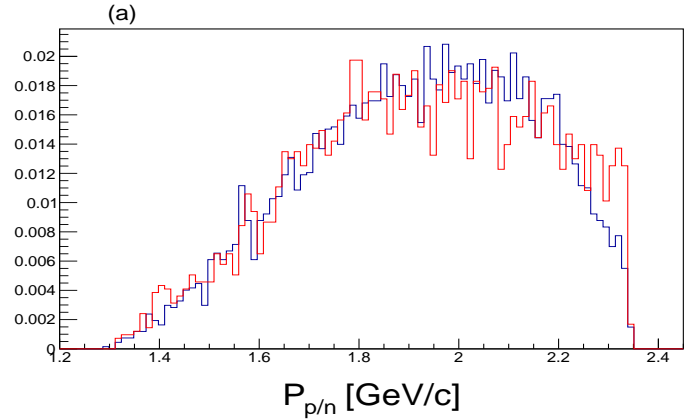

(b)
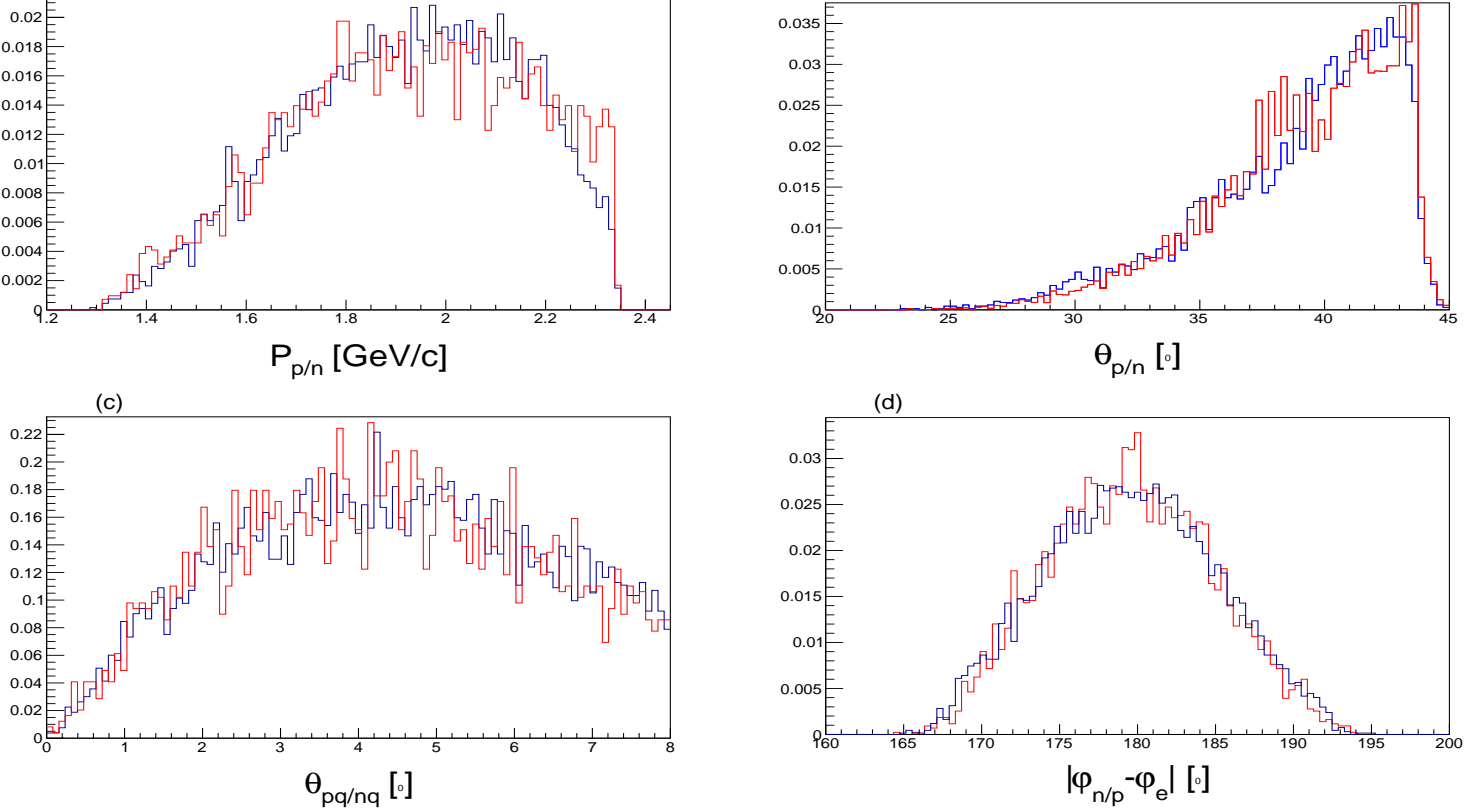

Figure 89: The smeared protons (red) and neutrons (blue) quantities after applying the QE cuts for ${ }^{27} \mathrm{Al}$ : (a) nucleon momentum, (b) scattering angle, (c) the angle between the nucleon and the momentum transfer vector, and (d) the absolute difference between the out of plane scattering angles of the nucleon and the electron. All distributions are normalized to unity such that only their shape is compared.

(a)
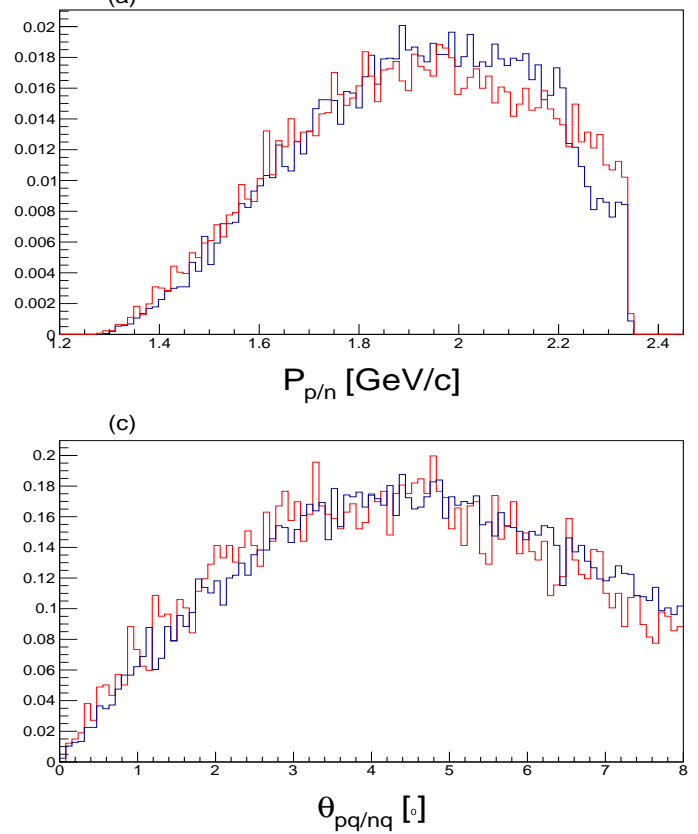

(b)

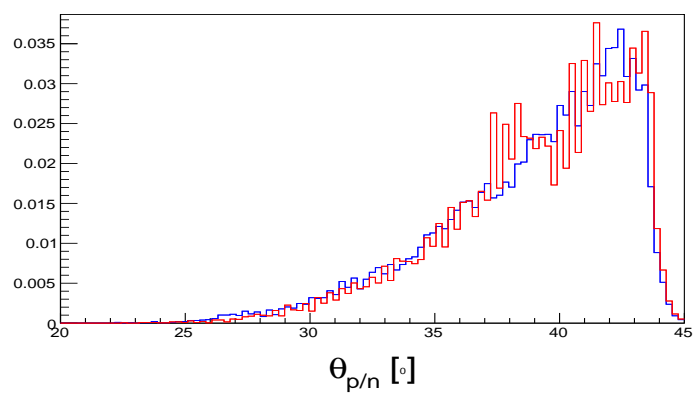

(d)

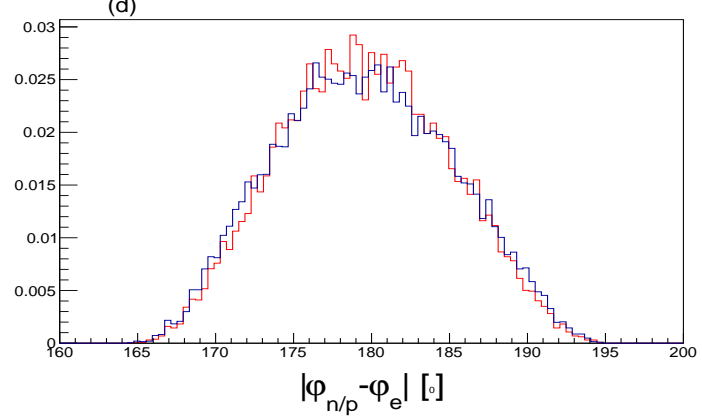

Figure 90: Same as Fig. 89 for ${ }^{56} \mathrm{Fe}$. 


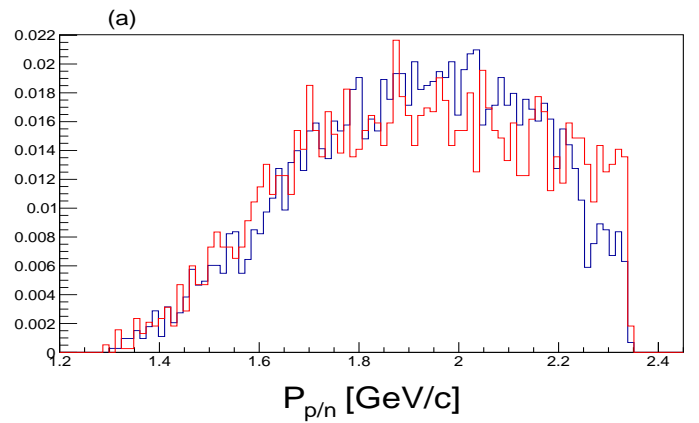

(b)
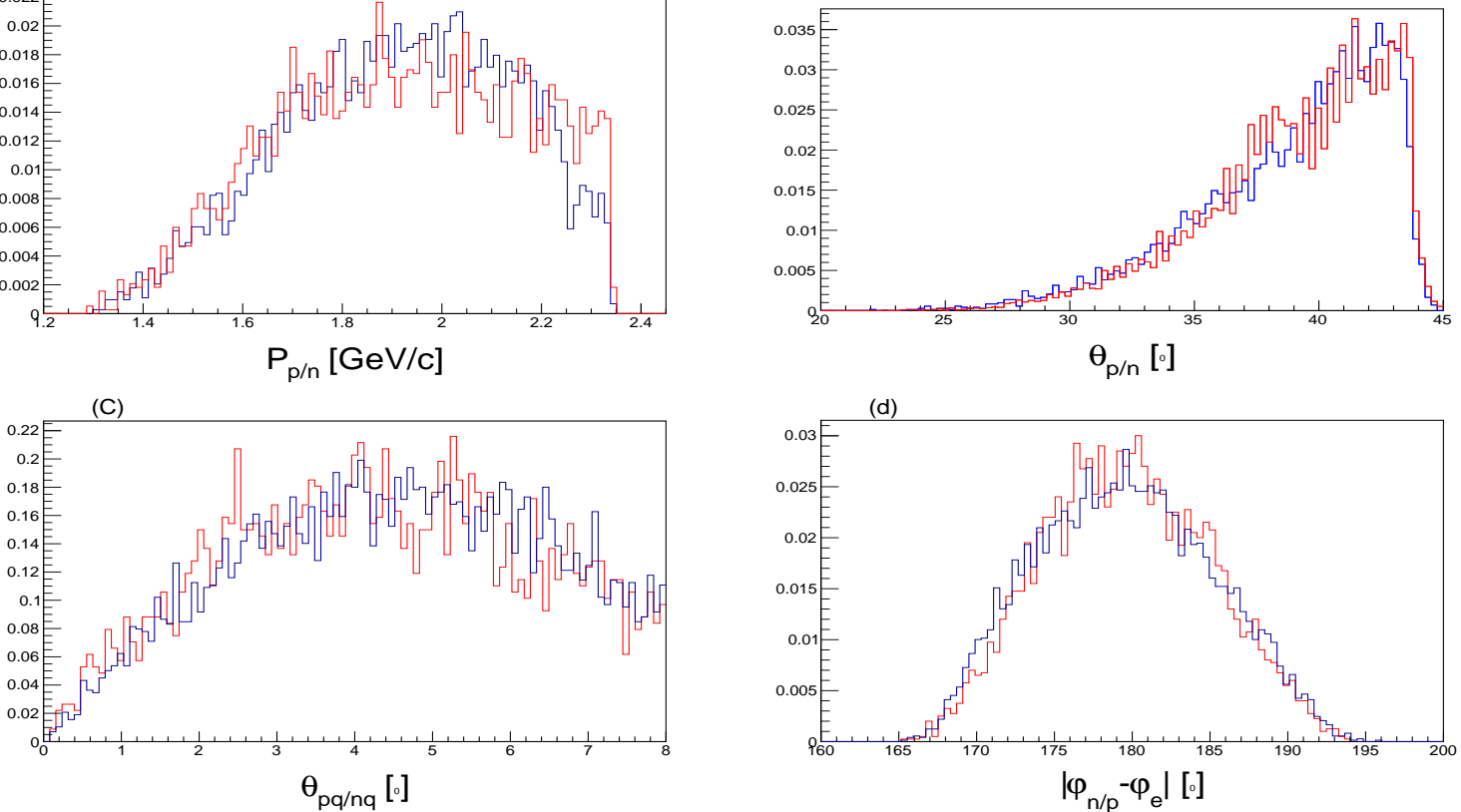

Figure 91: Same as Fig. 89 for ${ }^{208} \mathrm{~Pb}$. 


\section{C $\quad{ }^{27} \mathrm{Al},{ }^{56} \mathrm{Fe}$, and ${ }^{208} \mathrm{~Pb}$ distributions (triple coincidence)}

In this section we show distribtuions presented in Sec. 3.3 for ${ }^{27} \mathrm{Al},{ }^{56} \mathrm{Fe}$, and ${ }^{208} \mathrm{~Pb}$.

Figures 92 - 94 show distributions of different electron, proton, and recoil proton variables that characterize the selected $A\left(e, e^{\prime} p_{\text {smeared }} p\right)$ events (same as Figs. 58- 60).
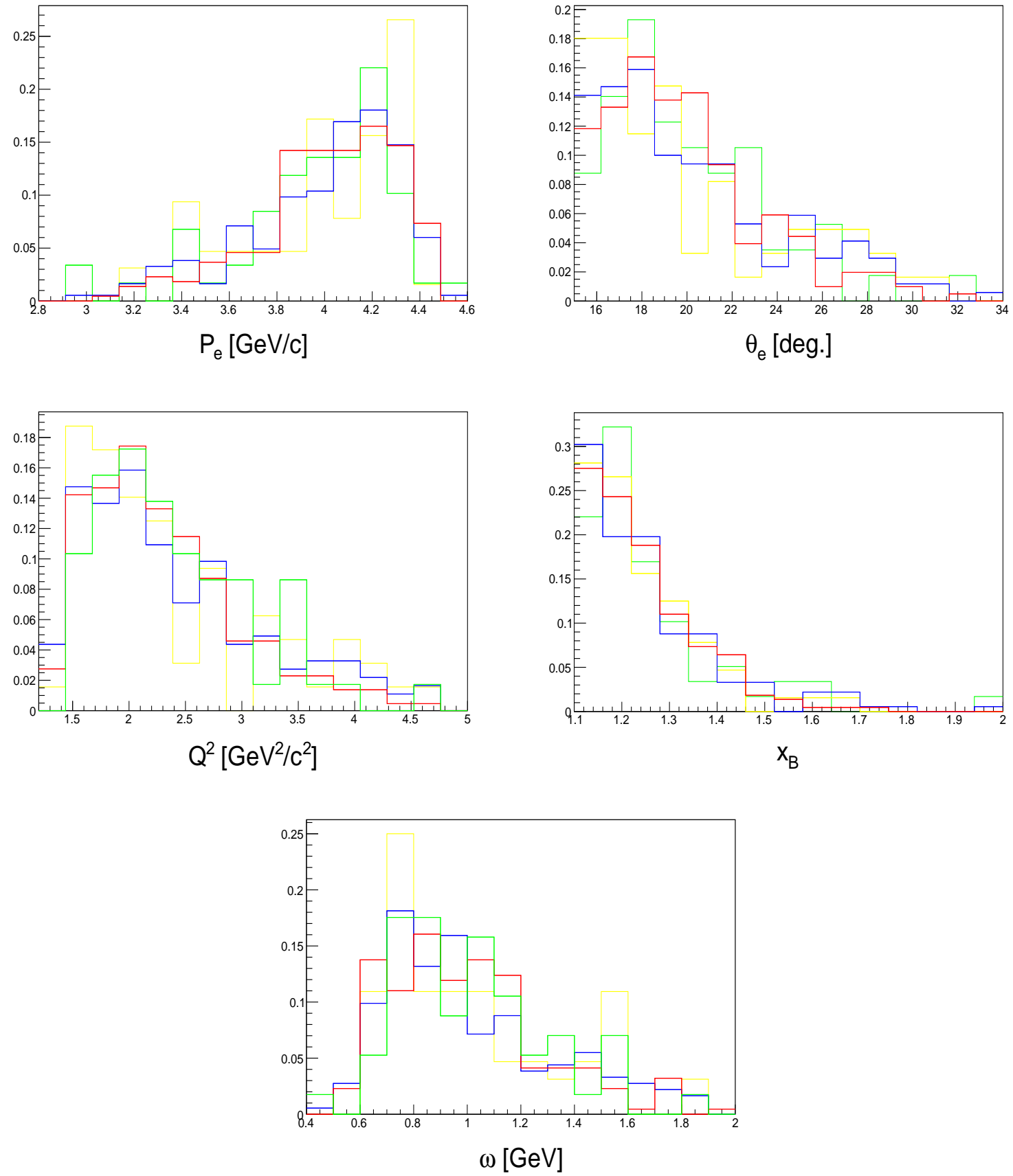

Figure 92: The electron kinematic variables for smeared $A\left(e, e^{\prime} p p\right)$ events. Blue - C, yellow - $\mathrm{Al}$, red - Fe, and green - Pb. Shown are the electron momentum, the electron scattering angle, $Q^{2}, x_{B}$, and $\omega$. All distributions are normalized to unity such that only their shapes are to be compared. 

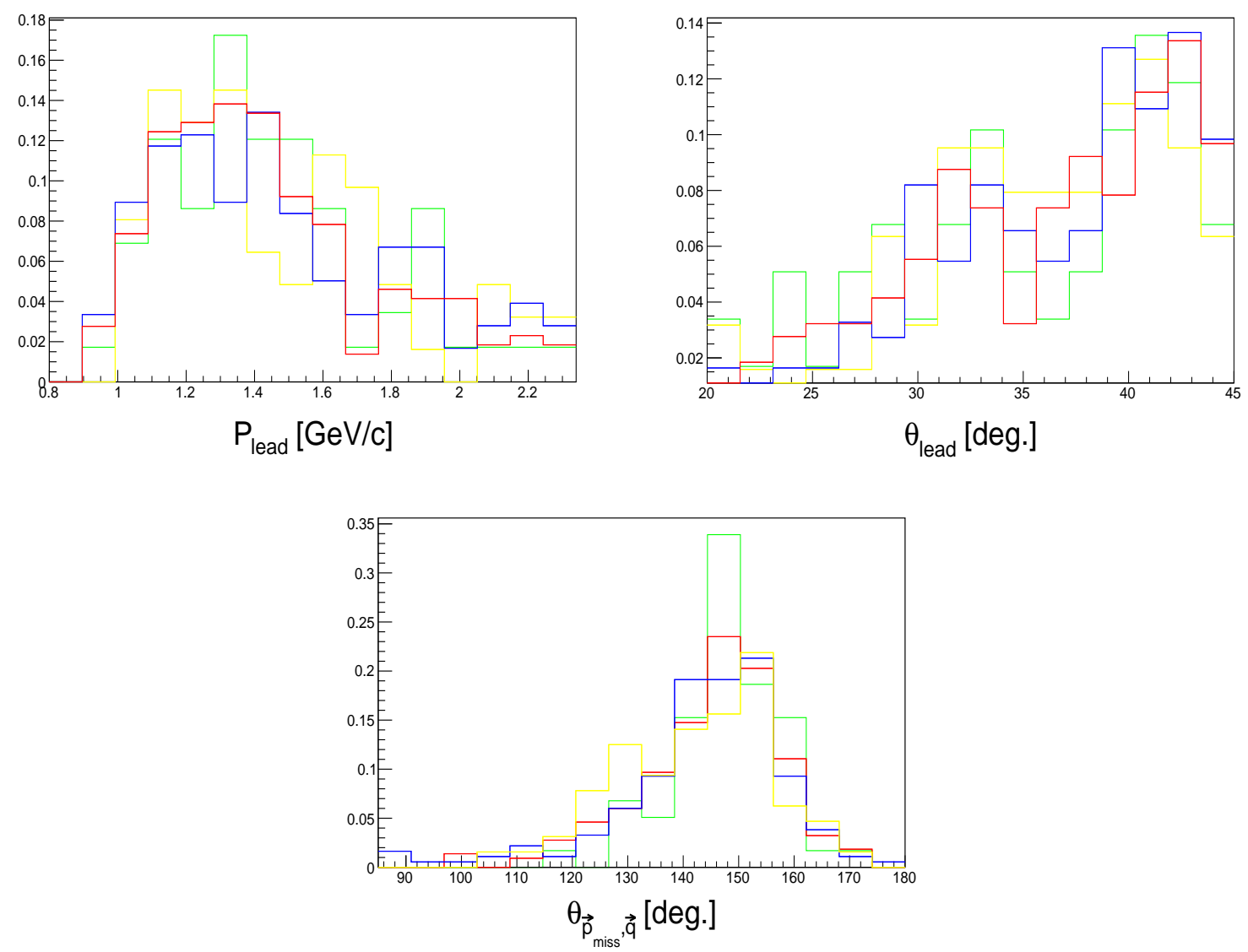

Figure 93: The proton kinematic variables for smeared $A\left(e, e^{\prime} p p\right)$ events. Blue - C, yellow - $\mathrm{Al}$, red - Fe, and green - $\mathrm{Pb}$. Shown are the proton momentum, the proton scattering angle, and the angle between the initial proton momentum $p_{\text {miss }}$ and the $q$ vector. All distributions are normalized to unity such that only their shapes are to be compared. 

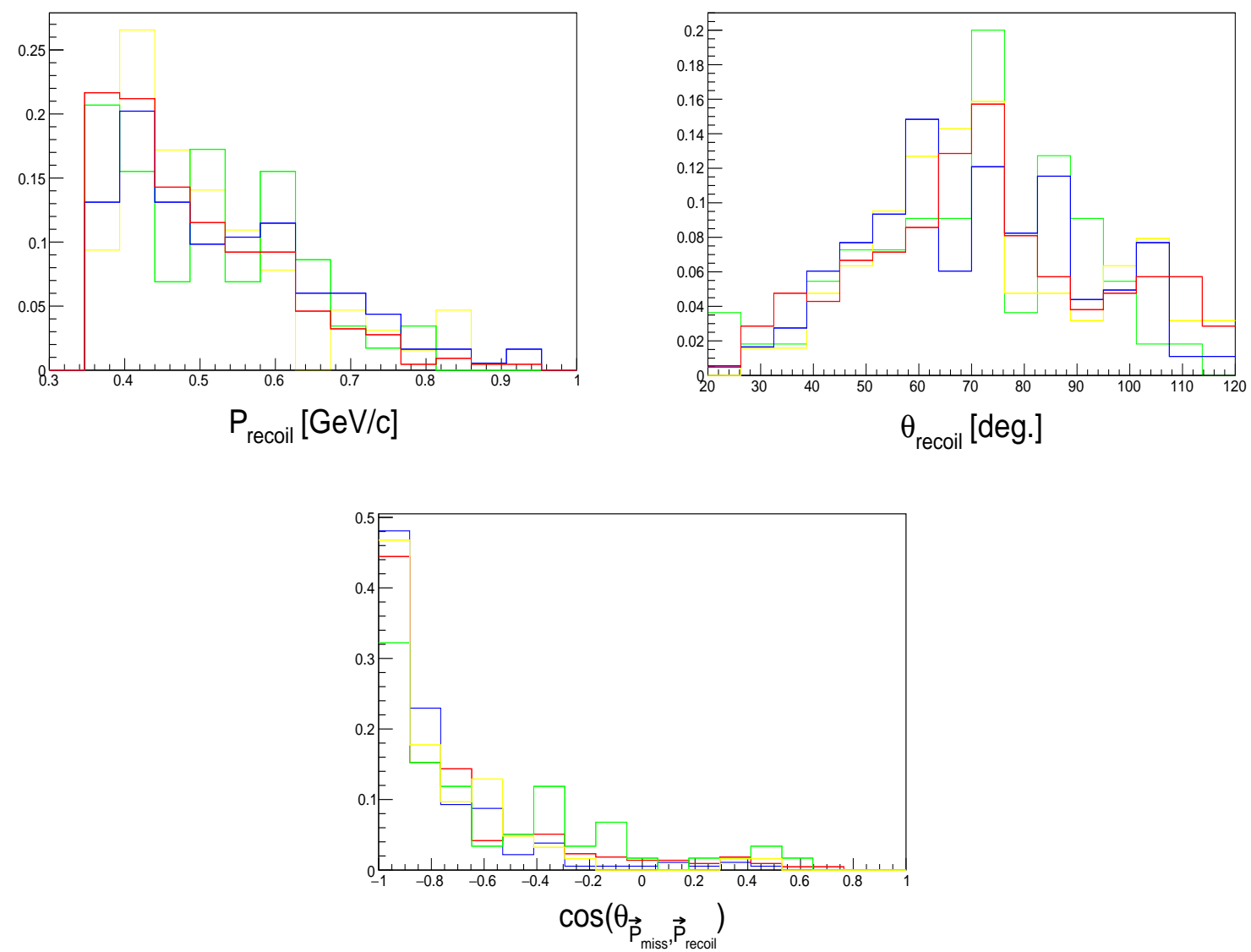

Figure 94: The recoil proton kinematic variables for smeared $A\left(e, e^{\prime} p p\right)$ events. Blue - C, yellow - Al, red - Fe, and green - $\mathrm{Pb}$. Shown are its momentum, its scattering angle, and the opening angle between the leading proton and the recoil proton. All distributions are normalized to unity such that only their shapes are to be compared. 


\section{SCX correction}

In the absence of FSI, assuming scattering from an $\mathrm{SRC}$, the $A\left(e, e^{\prime} n p\right)$ and $A\left(e, e^{\prime} p p\right)$ measured cross-sections can be written as:

$$
\begin{gathered}
\sigma_{A\left(e, e^{\prime} p p\right)}=\# p p_{A} \cdot 2 \cdot \sigma_{p} \\
\sigma_{A\left(e, e^{\prime} n p\right)}=\# n p_{A} \cdot \sigma_{n}
\end{gathered}
$$

where $\# p p_{A}\left(\# n p_{A}\right)$ is the number of proton-proton (neutron-proton) pairs in nucleus $A$ and $\sigma_{p}$ $\left(\sigma_{n}\right)$ is the electron-proton (electron-neutron) cross-section.

With FSI, one should take into account contributions from all $N N$-SRC pairs that can lead to the same measured final state, the effects of nuclear transparency and SCX. Using the notation defined in Sec. 4.4 for the SCX probabilities, Eq. 21 can be written as:

$$
\begin{gathered}
\sigma_{A\left(e, e^{\prime} p p\right)} \propto \# p p_{A} \cdot 2 \cdot \sigma_{p} \cdot P_{A}^{p p} \cdot T_{A, p p}+\# n p_{A} \cdot \sigma_{n} \cdot p_{A}^{[n] p} \cdot T_{A}^{*}+\# p n_{A} \cdot \sigma_{p} \cdot P_{A}^{p[n]} \cdot T_{A}^{*} \\
\sigma_{A\left(e, e^{\prime} n p\right)} \propto \# n p_{A} \cdot \sigma_{n} \cdot P_{A}^{n p} \cdot T_{A, n p}+\# p p_{A} \cdot 2 \cdot \sigma_{p} \cdot P_{A}^{[p] p} \cdot T_{A}^{*}+\# n n_{A} \cdot 2 \cdot \sigma_{n} \cdot P_{A}^{n[n]} \cdot T_{A}^{*}
\end{gathered}
$$

where $T_{p p}\left(T_{n p}\right)$ is the nuclear transparency for two protons (neutron-proton). $T^{*}$ is the transparency associated with a SCX process. Eq. 15 in Sec. 4.5 can be obtained from Eq. 22 by forming the $A\left(e, e^{\prime} p p\right) / A\left(e, e^{\prime} n p\right)$ ratio and assuing that $T^{*}=\frac{1}{2}\left(T_{p p}+T_{n p}\right)=T_{p p}=T_{n p}$. The latter approximation is valid when considering high- $Q^{2}$ reactions with a high-energy leading proton/neutron that has the same nuclear transparency for $p p$ and $n p$ pairs [28, 29].

The evaluation of Eq. 15 and the estimation of its uncertainties was done following [6], using a Monte-Carlo technique where its PDF was extracted from repeated calculations using different input values. In each calculation the values of the different parameters (experimental crosssection, SCX probabilities etc.) were randomly chosen from a Gaussian distribution centered at the measured or calculated value with width $(1 \sigma)$ that equaled their associated uncertainties. The cross-section ratios, $R$, are listed in Table 19 , the SCX probabilities are listed in Table 21 (based on the calculations of Ref. [28]), and for the kinematics of the current measurement $\sigma_{p / n}=\sigma_{p} / \sigma_{n}=2.30 \pm 0.15$. For asymmetric nuclei, $\eta_{A}=\frac{\# n n_{A}}{\# p p_{A}}$ was drawn from a uniform distribution between unity and the combinatorial ratio of possible $n n$ and $p p$ pairs in a given asymmetric nucleus. The resulting FSI-corrected $p p / n p$ SRC-pairs ratio are listed in Table 19 and were obtained from the PDF by taking its most probable value and estimating its confidence limits by integrating the PDF around this value, see Fig. 95 . 


\begin{tabular}{|c|c|c|c|c|}
\hline & $\mathrm{C}$ & $\mathrm{Al}$ & $\mathrm{Fe}$ & $\mathrm{Pb}$ \\
\hline \hline$P^{p p}$ & $0.908 \pm 0.006$ & $0.897 \pm 0.009$ & $0.891 \pm 0.010$ & $0.860 \pm 0.013$ \\
\hline$P_{A}^{[p] p}$ & $0.041 \pm 0.003$ & $0.046 \pm 0.004$ & $0.048 \pm 0.005$ & $0.059 \pm 0.006$ \\
\hline$P_{A}^{p[p]}$ & $0.048 \pm 0.003$ & $0.054 \pm 0.005$ & $0.057 \pm 0.006$ & $0.074 \pm 0.007$ \\
\hline$P_{A}^{[p p]}$ & $0.003 \pm 0.0002$ & $0.004 \pm 0.0003$ & $0.0042 \pm 0.0003$ & $0.007 \pm 0.0006$ \\
\hline$P_{A}^{p[n]}$ & $0.041 \pm 0.003$ & $0.047 \pm 0.005$ & $0.047 \pm 0.005$ & $0.047 \pm 0.005$ \\
\hline$P_{A}^{[p] n}$ & $0.035 \pm 0.002$ & $0.043 \pm 0.004$ & $0.046 \pm 0.005$ & $0.061 \pm 0.006$ \\
\hline$P_{A}^{n p p}$ & $0.922 \pm 0.005$ & $0.907 \pm 0.008$ & $0.903 \pm 0.009$ & $0.887 \pm 0.010$ \\
\hline$P_{A}^{[n] p}$ & $0.035 \pm 0.002$ & $0.040 \pm 0.004$ & $0.040 \pm 0.004$ & $0.040 \pm 0.004$ \\
\hline$P_{A}^{n[p]}$ & $0.041 \pm 0.003$ & $0.051 \pm 0.005$ & $0.054 \pm 0.006$ & $0.072 \pm 0.008$ \\
\hline$P_{A}^{[n p]}$ & $0.002 \pm 0.0001$ & $0.003 \pm 0.0002$ & $0.004 \pm 0.0003$ & $0.005 \pm 0.0004$ \\
\hline$P_{A}^{n[n]}$ & $0.048 \pm 0.003$ & $0.050 \pm 0.005$ & $0.049 \pm 0.005$ & $0.048 \pm 0.005$ \\
\hline
\end{tabular}

Table 21: The SCX probabilities for different $N N$ pairs and nuclei.
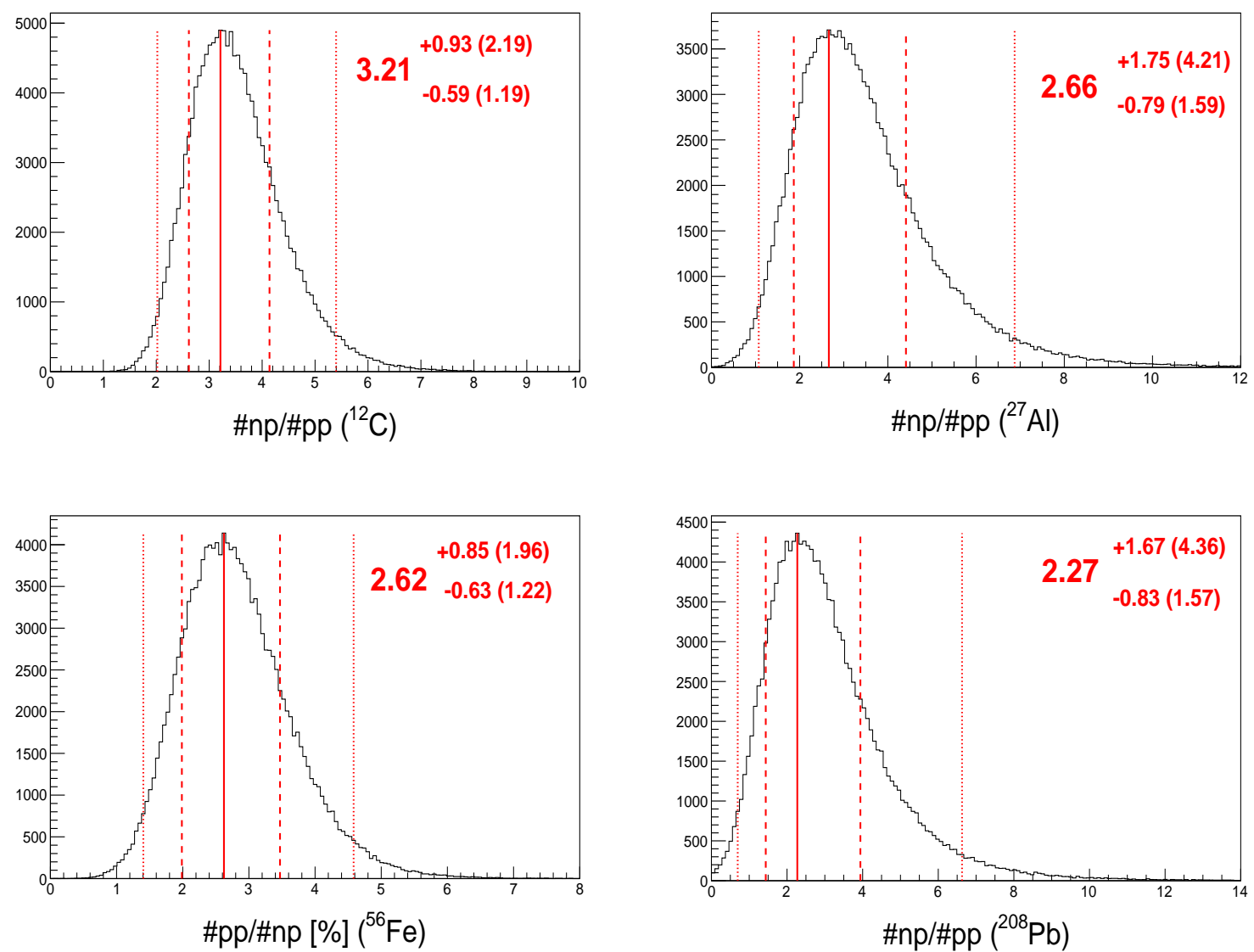

Figure 95: The PDF distribution of the $p p / n p$ ratio. The solid red line marks the median value while the coarse dashed and fine red lines mark the regions of $68 \%$ and $95 \%$ confidence level, respectively. See text for details. 
אוניברסיטת TEL AVIV

תל אביב UNIVERSITY

\section{קורלציות קצרות טווח בין זוגות נוקליאונים בגרעינים כבדים}

חיבור לשם קבלת התואר "דוקטור לפילוסופיה"

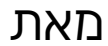

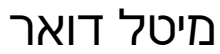

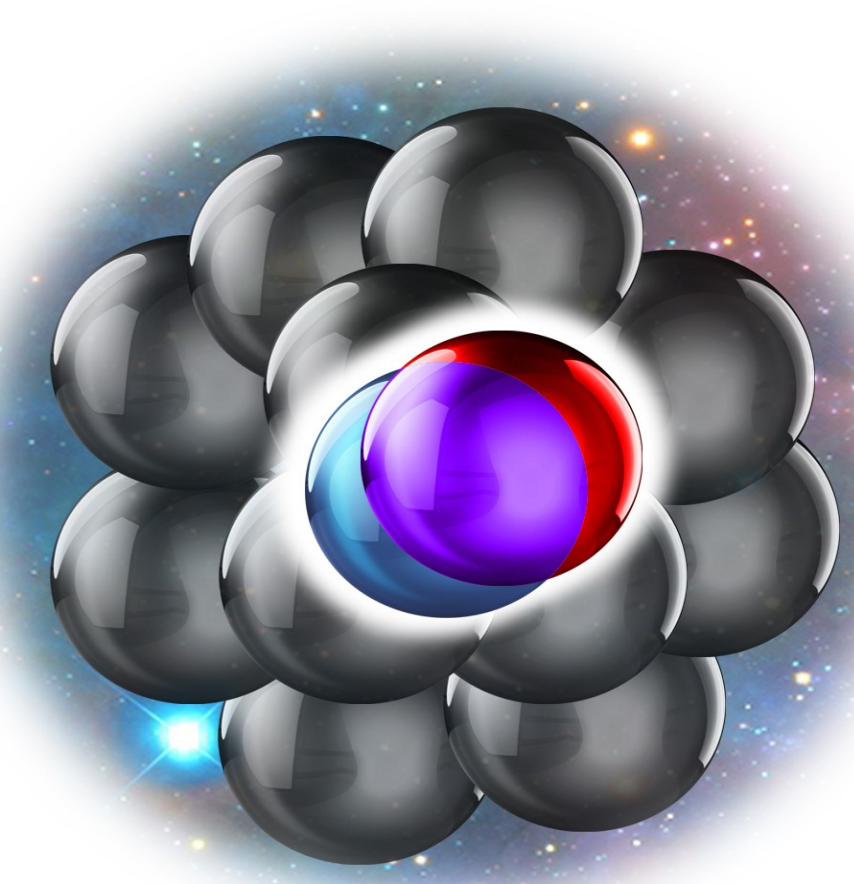

הוגש לסנאט של אוניברסיטת תל אביב

דצמבר 2018

עבודה Iו נעשה בהנחייתו של

פרופ' אלי פיסצקי 
גרעיני האטומים מהווים כמעט את כל המסה ביקום. גרעין האטום הינו אחת מהת מהמערכות הקוונטיות המיות הצפופות

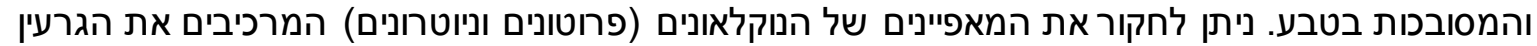

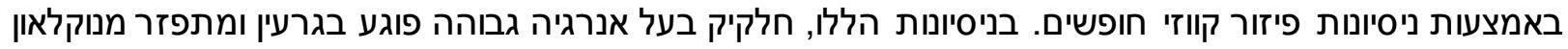

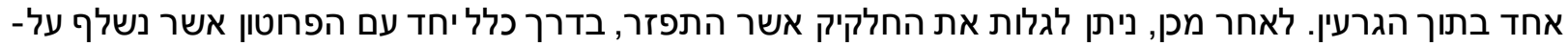

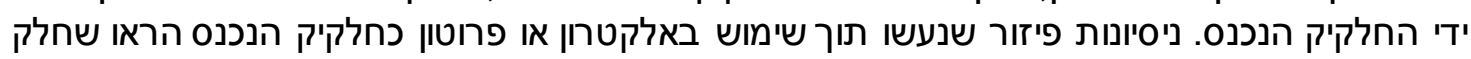

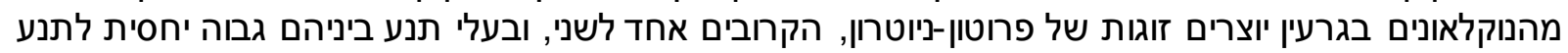

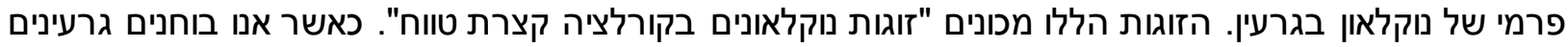

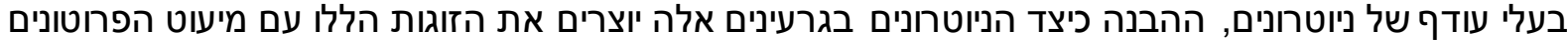

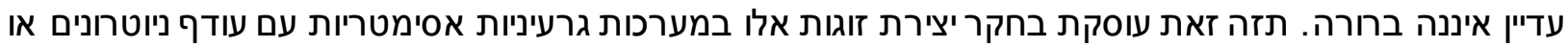
פרוטונים.

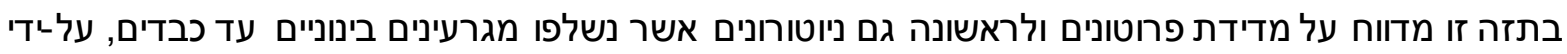

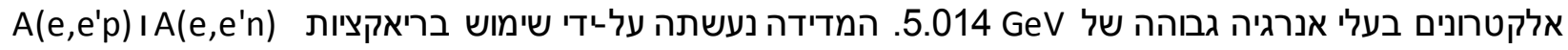

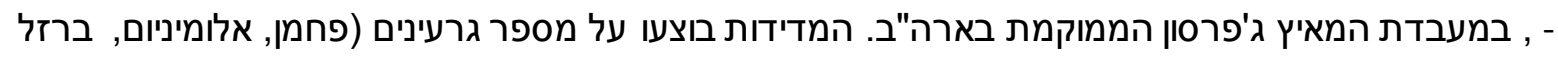

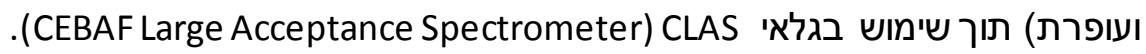

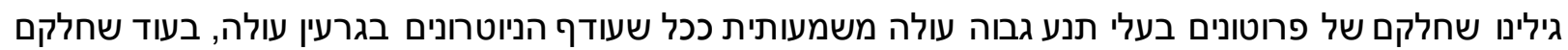

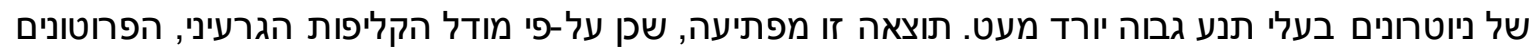

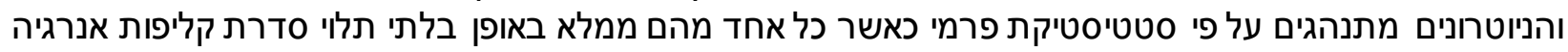
שונות.

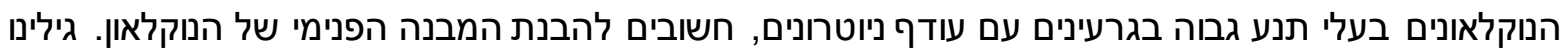

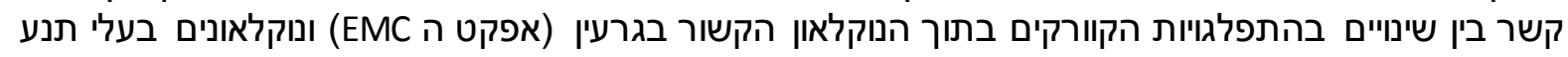

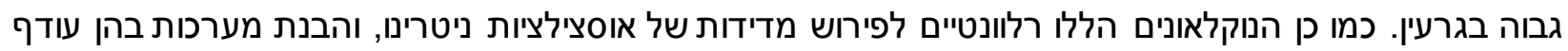
הניוטרונים הינו רב, כגון כוכבי ניוטרונים הלוים

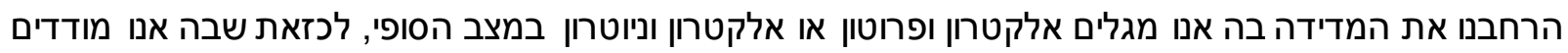

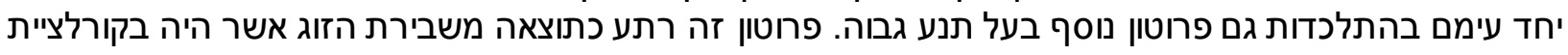

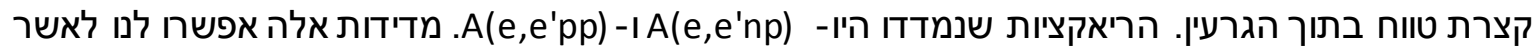

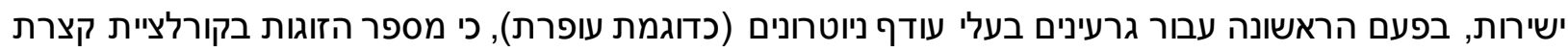

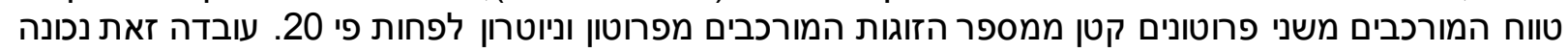
עבור כל הגרעינים שנמדדו ללא תלות בעודף ממודף הניוטרונים בהות המרים. 